archives-ouvertes

\title{
Design of a new neutron delivery system for high flux source
}

Romain Boffy

\section{To cite this version:}

Romain Boffy. Design of a new neutron delivery system for high flux source. Materials. Universidad

Politécnica de Madrid; Institut Laue-Langevin, Grenoble, France, 2016. English. < tel-01358632>

\section{HAL Id: tel-01358632 \\ https://hal.archives-ouvertes.fr/tel-01358632}

Submitted on 3 Oct 2016

HAL is a multi-disciplinary open access archive for the deposit and dissemination of scientific research documents, whether they are published or not. The documents may come from teaching and research institutions in France or abroad, or from public or private research centers.
L'archive ouverte pluridisciplinaire HAL, est destinée au dépôt et à la diffusion de documents scientifiques de niveau recherche, publiés ou non, émanant des établissements d'enseignement et de recherche français ou étrangers, des laboratoires publics ou privés. 


\section{TESIS DOCTORAL}

\section{DESIGN OF A NEW NEUTRON DELIVERY SYSTEM FOR HIGH FLUX SOURCE}

TESIS DOCTORAL PARA LA OBTENCIÓN DEL TÍTULO DE DOCTOR
FEBRERO 2016

Romain Boffy

DIRECTORES DE LA TESIS DOCTORAL:

Francisco Javier Bermejo Barrera Jose Manuel Perlado Martín 



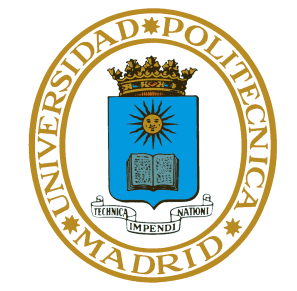

\title{
Design of a new neutron delivery system for high flux source
}

\author{
Romain BOFFY \\ Ingeniero energético y nuclear \\ by Institut Polytechnique de Grenoble
}

Thesis Directors:

Dr. Francisco Javier Bermejo Barrera Consejo Superior de Investigaciones Científicas

Dr. Jose Manuel Perlado Martín Instituto de Fusión Nuclear 

Tibunal nombrado por el Sr.

Rector Magnífico de la

Universidada Politécnica de Madrid, el 5 de Febrero de 2016

Presidente: Dr Felix Fernandez Alonso (RAL)

Secretario: Dr Antonio Juan Rivera de Mena (IFN - UPM)

Vocal: Dr Thibault Charpentier (CEA)

Vocal: Dr Joaquin Cobos Sabaté (CIEMAT)

Vocal: Prof Carlos Cabrillo (CSIC)

Suplente: Dr Fernando Sordo (ESS-Bilbao)

Suplente: Dr Josep LLuis Tamarit (UPC)

Realizado el acto de defensa y lectura de la tesis en día 5 de Febrero de 2016 en la Escuela Técnica Superior de Ingenieros Industriales.

Calificación : Sobresaliente Cum Laude

EL PRESIDENTE

LOS VOCALES

EL SECRETARIO 



\section{Acknowledgments}

This whole project would not have been possible without Jérôme Beaucour and Jose Luis Martinez, who accepted me first as a trainee for a neutron guide design internship and then as a Ph.D. student on the related topic of mirror substrate study. The main work partner I had throughout these years was Michael Kreuz who has always been available to discuss scientific studies whether they were from his field of expertise or not, thank you very much. From the same ILL service, I would like to thank Giuliana Manzin and Benjamin Giroud, who advised me whenever I needed it.

From the reactor division, I would like to especially thank Marc Samuel and his team, Elvio Mannino, Cédric Laurent and Renaud Silvestre, who assisted me on the T4 irradiations of this study. Also, I cannot forget Xavier Fargues, Frédérique Fréry, Véronique Caillot, and Bruno Desbrière for their help at some point on the hard path to send samples to irradiation in the ILL High Flux Reactor.

In the same vein, I wish to thank Ulli Köster, who advised me a lot on in-situ flux monitoring with Zirconium foils and the perturbed flux issue. His explanations on various topics ranging from gamma activation analysis to radioactive material transport were always useful. In terms of radio-protection, I am happy to thank the constant help from Gilles Rignon and his team, Patrice Cochet, Ian Sives, Sebastion Grimaud, Olivier Duny, Fabrice Rencurel, Rob Dixon, Mark Giltrow, Denis Boulicault and David Roberts.

Here comes the large spectrum acknowledgements for everyone at ILL who assisted me directly or indirectly: people from the Hall d'essais, Bureau d'études, Valérie Duchasténier, Labo vide, Duncan Atkins, Christophe Mounier, Stéfane Fuard and BPC in general, Sergio Martinez, Instruments responsibles such as Ralf Schweins and Henry Fischer, Thierry Bigault, decontaminators, Irina Snigireva (ESRF), Bruno Guérard, IT services, Jaime Segura, Alain Lechat, and of course, ILL staff in general for their warm welcome.

Of course, I had the great support from my scientific supervisor, Professor Javier Bermejo, especially the last year when his manuscript correction velocity was probably the highest any student could dream. Talking about tail wind, most of what is presented in the document would not have been possible without Dr. Charles Simon, who put me in contact with Dr. Marie Foret from Laboratoire Charles Coulomb at Montpellier. The discussions with her team allowed to pinpoint the need to collaborate with the French nuclear glass specialists at CEA Marcoule. It is for that reason that I met Sylvain Peuget, Jean-Marc Delaye, Olivier Bouty, and Christophe Jégou. In this way, I deeply acknowledge Sylvain Peuget for his help on borosilicate glass characterisation techniques, especially Raman, as well as borosilicate structure in general. Sylvain was definitively the most important person I met for the scientific development of my work. I want to 
acknowledge Joe Somers and Laura Martel for having accepted to do NMR experiments on my irradiated samples at ITU.

I want to thank the Departamento de Ingenierial Nuclear and the Instituto de Fusión Nuclear from UPM for their support of this work.

Finally, I think blossoming at work also utterly depends on where your roots are grounded. In my case, the soil was rich thanks to all of you: Cécile, Guillaume, Jean, JJ, Laurent, les animaux de Toulouse et d'ailleurs, les Lyonnais, les [Re] en général, Louise, Méline, mes parents et ma sœur, Pauline, Philippe, Rémi C, Rémi D, Romain, Simon et aussi Alison, Anne, Francisco, Patrizia and Stéfanos. I cannot forget the person with whom I shared my life throughout these years, my best team mate Géraldine. With her I always found comfort, gentleness and wisdom. 


\section{Resumen}

La construcción en la actualidad de nuevas fuentes para el uso de haces de neutrones así como los programas de renovación en curso en algunas de las instalaciones experimentales existentes han evidenciado la necesidad urgente de desarrollar la tecnología empleada para la construcción de guías de neutrones con objeto de hacerlas mas eficientes y duraderas. Esto viene motivado por el hecho de que varias instalaciones de experimentación con haces de neutrones han reportado un número de incidentes mecánicos con tales guías, lo que hace urgente el progresar en nuestro conocimiento de los susbtratos vítreos sobre los cuales se depositan los espejos que permiten la reflexión total de los neutrones y como aquellos se degradan con la radiación. La presente tesis se inscribe en un acuerdo de colaboración establecido entre el Institut Max von Laue - Paul Langevin (ILL) de Grenoble y el Consorcio ESS-Bilbao con objeto de mejorar el rendimiento y sostenibilidad de los sistemas futuros de guiado de neutrones. El caso de la Fuente Europea de Espalación en construcción en Lund sirve como ejemplo ya que se contempla la instalación de guías de neutrones de más de 100 metros en algunos de los instrumentos. Por otro lado, instalaciones como el ILL prevén también dentro del programa Endurance de rejuvenecimiento la reconstrucción de varias líneas de transporte de haz.

Para el presente estudio se seleccionaron cuatro tipos de vidrios borosilicatados que fueron el Borofloat, N-ZK7, N-BK7 y SBSL7. Los tres primeros son bien conocidos por los especialistas en instrumentación neutrónica ya que se han empleado en la construcción de varias instalaciones mientras que el último es un candidato potencial en la fabricación de substratos para espejos neutrónicos en un futuro. Los cuatro vidrios tiene un contenido en óxido de Boro muy similar, approximadamente un 10 mol.\%. Tal hecho que obedece a las regulaciones para la fabricación de estos dispositivos hace que tales substratos operen como protección radiológica absorbiendo los neutrones transmitidos a través del espejo de neutrones. Como contrapartida a tal beneficio, la reacción de captura ${ }^{10} \mathrm{~B}(\mathrm{n}, \alpha)^{7} \mathrm{Li}$ puede degradar el substrato vítreo debido a los $2.5 \mathrm{MeV}$ de energía cinética depositados por la partícula $\alpha$ y los núcleos en retroceso y de hecho la fragilidad de tales vidrios bajo radiación ha sido atribuida desde hace ya tiempo a los efectos de esta reacción.

La metodología empleada en esta tesis se ha centrado en el estudio de la estructura de estos vidrios borosilicatados y como esta se comporta bajo condiciones de radiación. Los materiales en cuestión presentan estructuras que dependen de su composición química y en particular del ratio entre formadores y modificadores de la red iono-covalente. Para ello se han empleado un conjunto de técnicas de caracterización tanto macro- como microscópicas tales como estudios de dureza, TEM, Raman, SANS etc. que se han empleado también para determinar el comportamiento de estos materiales bajo radiación. En par- 
ticular, algunas propiedades macroscópicas relacionadas con la resistencia de estos vidrios como elementos estructurales de las guías de neutrones han sido estudiadas así como también los cambios en la estructura vítrea consecuencia de la radiación. Para este propósito se ha diseñado y fabricado por el ILL un aparato para irradiación de muestras con neutrones térmicos en el reactor del ILL que permite controlar la temperatura alcanzada por la muestra a menos de $100{ }^{\circ} \mathrm{C}$. Tal equipo en comparación con otros ya existences permite en cuestión de dias acumular las dosis recibidas por una guía en operación a lo largo de varios años.

El uso conjunto de varias técnicas de caracterización ha llevado a revelar que los vidrios aqui estudiados son significativamente diferentes en cuanto a su estructura y que tales diferencias afectan a sus propiedades macroscópicas asi como a su comportamiento bajo radiación. Tal resultado ha sido sorprendente ya que, como se ha mencionado antes, algunos de estos vidrios eran bien conocidos por los fabricantes de guías de neutrones y hasta el momento eran considerados prácticamente similares debido a su contenido comparable en óxido de Boro. Sin embargo, los materiales N-BK7 and S-BSL7 muetran gran homogeneidad a todas las escalas de longitud, y más específicamente, a escalas nanométricas las subredes de Sílice y óxido de Boro se mezclan dando logar a estructuras locales que recuerdan a la del cristal de Reedmergnerita. Por el contrario, N-ZK7 y Borofloat muestran dominios separados ricos en Sílice o Boro.

Como era de esperar, las importantes diferencias arriba mencionadas se traducen en comportamientos dispares de estos materiales bajo un haz de neutrones térmicos. Los resultados muestran que el N-BK7 y el S-BSL7 son los más estables bajo radiación, lo que macroscópicamente hace que estos materiales muestren un comportamiento similar expandiéndose lentamente en función de la dosis recibida. Por el contario, los otros dos materiales muestran un comportamiento mucho más reactivo, que hace que inicialmente se compacten con la dosis recibida lo que hace que las redes de Silicio y Boro se mezclen resultando en un incremento en densidad hasta alcanzar un valor límite, seguido por un proceso de expansión lenta que resulta comparable al observado para N-BK7 y SBSL7. Estos resultados nos han permitido explicar el origen de las notorias diferencias observadas en cuanto a las dosis límite a partir de las cuales estos materiales desarrollan procesos de fragmentación en superficie. 


\section{Abstract}

The building of new experimental neutron beam facilities as well as the renewal programmes under development at some of the already existing installations have pinpointed the urgent need to develop neutron guide technology in order to make such neutron transport devices more efficient and durable. In fact, a number of mechanical failures of neutron guides have been reported by several research centres. It is therefore important to understand the behaviour of the glass substrates on top of which the neutron optics mirrors are deposited, and how these materials degrade under radiation conditions. The case of the European Spallation Source (ESS), at present under construction at Lund, is a good example. It previews the deployment of neutron guides having more than 100 metres of length for most of the instruments. Also, the future renovation programme of the ILL, called Endurance, foresees the refurbishment of several beam lines. This Ph.D. thesis was the result of a collaboration agreement between the ILL and ESS-Bilbao, aiming to improve the performance and sustainability of future neutron delivery systems.

Four different industrially produced alkali-borosilicate glasses were selected for this study: Borofloat, N-ZK7, N-BK7, and S-BSL7. The first three are well known within the neutron instrumentation community, as they have already been used in several installations; whereas the last one is, at present, considered a candidate for making future mirror substrates. All four glasses have a comparable content of boron oxide of about 10 mol.\%. The presence of such a strong neutron absorption element is in fact a mandatory component for the manufacturing of neutron guides, because it provides a radiological shielding for the environment. This benefit is, however, somewhat counterbalanced, since the resulting ${ }^{10} \mathrm{~B}(\mathrm{n}, \alpha){ }^{7} \mathrm{Li}$ reactions degrade the glass due to the deposited energy of $2.5 \mathrm{MeV}$ by the $\alpha$ particle and the recoil nuclei. In fact, the brittleness of some of these materials has been ascribed to this reaction.

The methodology employed by this study consisted in understanding the general structure of borosilicates and how they behave under irradiation. Such materials have a microscopic structure strongly dependent upon their chemical content and particularly on the ratios between network formers and modifiers. The materials have been characterized by a suite of macroscopic and structural techniques such as hardness, TEM, Raman, SANS, etc, and their behaviour under irradiation was analysed. Macroscopic properties related to their resistance when used as guide structural elements were monitored. Also, changes in the vitreous structure due to radiation were observed by means of several experimental tools. For this purpose, an irradiation apparatus has been designed and manufactured to enable irradiation with thermal neutrons within the ILL reactor while keeping the samples below $100{ }^{\circ} \mathrm{C}$. The main advantage of this equipment, compared to 
others previously available, was that it enabled the glass to reach an equivalent neutron dose to that accumulated after several years of use as guides, in just few days.

The concurrent use of complementary characterization techniques lead to the discovery that the studied glasses were deeply different in terms of their glass network. This had a strong impact on their macroscopic properties and their behaviour under irradiation. This result was a surprise since, as stated above, some of these materials were well known by the neutron guide manufacturers, and were considered to be almost equivalent because of their similar boron oxide content. The N-BK7 and S-BSL7 materials appear to be fairly homogeneous glasses at different length scales. More specifically, at nanometre scales, silicon and boron oxide units seem to mix and generate larger structures somewhat resembling crystalline Reedmergnerite. In contrast, N-ZK7 and Borofloat are characterized by either silicon or boron rich domains.

As one could expect, these drastic differences lead to their behaviour under thermal neutron flux. The results show that N-BK7 and S-BSL7 are structurally the most stable under radiation. Macroscopically, such stability results in the fact that these two materials show very slow swelling as a function of radiation dose. In contrast, the two other glasses are much more reactive. The whole glass structure compacts upon radiation. Specifically, the silica network and the boron units tend to blend, leading to an increase in density up to some saturation, followed by a very slow expansion of the same order as shown by N-BK7 and S-BSL7. Such findings allowed us to explain the drastic differences in the radiation limits for macroscopic surface splintering for these materials when they are used in neutron guides. 


\section{Contents}

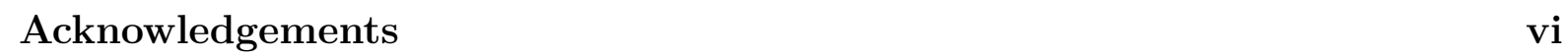

Resumen viii

Abstract $\quad x$

Contents $\quad$ xiii

Abbreviations $\quad$ xiv

\begin{tabular}{ll} 
Computer programs employed & Xv \\
\hline
\end{tabular}

Introduction 1

1 Fundamentals and Problem setting 3

1.1 Introduction to neutron reflection theory . . . . . . . . . . . . . 3

1.2 Neutron guide basic features - examples in large research facilities . . . . 6

1.3 Beam wave propagation theory and losses in substrates . . . . . . . . . . 9

1.4 Improving the design of neutron guides . . . . . . . . . . . . . . . 13

1.5 Guide ageing and reported incidents . . . . . . . . . . . . . . 15

$\begin{array}{llr}2 & \text { Radiation effects in glass structure } & 20\end{array}$

2.1 What is glass? . . . . . . . . . . . . . . . . . . . 20

2.2 Chemical composition of the studied glasses . . . . . . . . . . . . . . . 23

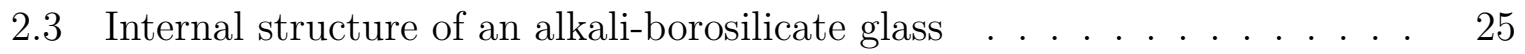

2.4 Sources of radiation damage: gamma rays, fast and moderated neutrons. 30

2.5 Glass behaviour under irradiation . . . . . . . . . . . . . . . . 31

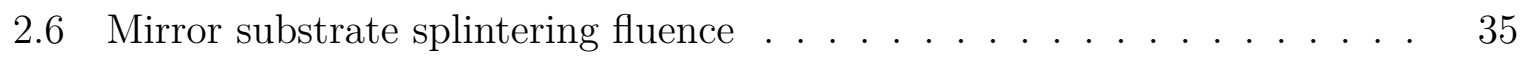

2.7 Ageing mechanism $\ldots \ldots \ldots \ldots \ldots$. . . . . . . . . . . . . . . 45

\begin{tabular}{|lll}
\hline 3 & Macroscopic characterization and irradiation program & 47
\end{tabular}

3.1 Differential scanning calorimetry . . . . . . . . . . . . . . . . . 47

3.2 Hardness and fracture toughness . . . . . . . . . . . . . . . . . . . . . 50

3.3 Electronic Microscopy Imaging. . . . . . . . . . . . . . . . . . . . . . . . 52

3.4 A new fast homogeneous irradiation equipment . . . . . . . . . . . . . 55

3.5 Gamma ray spectroscopy . . . . . . . . . . . . . . . . . . 65

3.6 Bulk density evolution under thermal neutron flux . . . . . . . . . . . . . 66

3.7 Stress generation in borosilicate glass by neutron irradiation . . . . . . . 71 
$4 \quad$ Vitreous structure analysis and response to thermal neutron irradiation 78

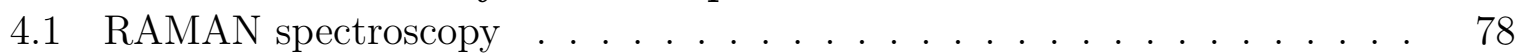

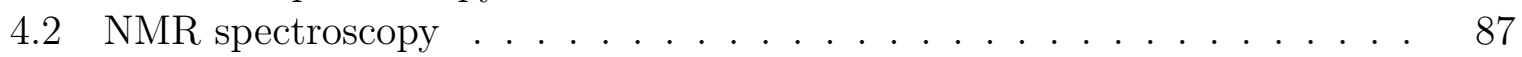

4.3 Neutron scattering $\quad \ldots \ldots \ldots \ldots$

4.4 Neutron diffraction . . . . . . . . . . . . . . . . . . . . . . . . . 111

4.5 Interpretation of the results . . . . . . . . . . . . . . 117

\begin{tabular}{lr}
\hline Conclusion & 123
\end{tabular}

\begin{tabular}{ll}
\hline List of Publications & 125
\end{tabular}

\begin{tabular}{ll}
\hline Appendices & 125
\end{tabular}

\begin{tabular}{|lr}
\hline A Neutron guide design & 126
\end{tabular}

A.1 Analytical estimations . . . . . . . . . . . . . . . . . . 126

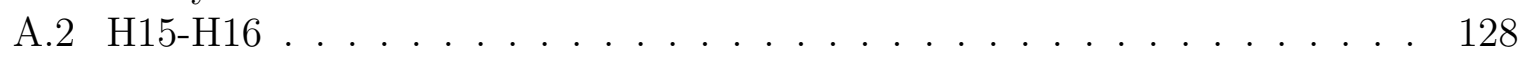

A.3 H24 . . . . . . . . . . . . . . . . . . . . . . . . . . . . . 137

B Presentation of some examples of the written computation code employed

Bibliography 


\section{Abbreviations}

$\begin{array}{ll}\text { ABS and BS } & - \text { Alkali-BoroSilicate and BoroSilicate } \\ \text { CT } & - \text { Central Transition } \\ \text { DSC } & - \text { Differential Scanning Calorimetry } \\ \text { ESS } & - \text { European Spallation Source } \\ \text { HAADF } & - \text { High-Angle Annular Dark Field } \\ \text { HCS } & - \text { Horizontal Cold Source } \\ \text { ILL } & - \text { Institut Max Von-Laue - Paul Langevin } \\ \text { MAS } & - \text { Magic Angle Spinning } \\ \text { NBO } & - \text { Non-Bridging Oxygen } \\ \text { NBS } & - \text { Sodium BoroSilicate } \\ \text { NMR } & - \text { Nuclear Magnetic Resonance } \\ \text { PDF } & - \text { Pair Distribution Function } \\ \text { HFR } & - \text { ILL's High Flux Reactor } \\ \text { RMS } & - \text { Root Mean Square } \\ \text { SANS } & - \text { Small Angle Neutron Scattering } \\ \text { SEM } & - \text { Scanning Electron Microscope } \\ \text { ST } & - \text { Satellite Transition } \\ \text { STEM } & - \text { Scanning Transmission Electron Microscope } \\ \text { TEM } & - \text { Transmission Electron Microscope } \\ \text { VCS } & - \text { Vertical Cold Source }\end{array}$




\section{Computer programs employed}

CASTOR: Tool developed at ILL that calculates the resulting activity from a given composition and neutron irradiation characteristics. It details every stable and unstable nuclei and outputs their activity in Becquerel units. Though, the software does not account for the sample self-shielding when elements with high cross-section are involved.

DMfit: Versatile tool designed to fit NMR spectra, including solid state NMR. It provides a variety of different models that account for: Gaussian/Lorentzian lines, spinning sidebands, Chemical Shift Anisotropy (static and MAS), first and second order Quadrupolar interaction (static and MAS), etc. More details can be found on the site http://nmr.cemhti.cnrs-orleans.fr/dmfit/, 71

McStas: Ongoing project to create a general tool for simulating neutron scattering instruments. The project is a collaboration between DTU Physics (formerly RIS $\varnothing$ DTU and RIS $\varnothing$ Natnl. Lab), University of Copenhagen, Paul Scherrer Institute and Institut Laue-Langevin. The software is freely available and can be downloaded from the site www.mcstas.org. McStas is based on a compiler that reads a highlevel specification language defining the instrument to be simulated and produces C code that performs the Monte Carlo Simulation. McStas supports triple-axis, time-of-flight instruments, and polarised neutrons. It comes with a comprehensive manual and a library of well-tested components that include most standard elements of neutron scattering instruments, including steady-state and pulsed sources, monochromators/analysers, guides, collimators, vanadium and powder samples, velocity selectors and choppers, and a variety of detectors. 109 60

MCNP: General-purpose Monte Carlo N-Particle code that can be used for neutron, photon, electron, or coupled neutron/photon/electron transport. Specific areas of application include, but are not limited to, radiation protection and dosimetry, radiation shielding, radiography, medical physics, nuclear criticality safety, Detector Design and analysis, nuclear oil well logging, Accelerator target design, Fission and fusion reactor design, decontamination and decommissioning. The code treats an arbitrary three-dimensional configuration of materials in geometric cells bounded by first- and second-degree surfaces and fourth-degree elliptical tori. More details can be found on the site https://mcnp.lanl.gov/.

Microshield: Comprehensive photon/gamma ray shielding and dose assessment program that is widely used for designing shields, estimating source strength from radiation measurements, minimizing exposure to people, and teaching shielding 
principles. CASTOR output file can be used as an input of Microshield. More details can be found on the site http://radiationsoftware.com/microshield/.

SASview: Software for the analysis of Small-Angle Scattering (SAS) data. It fits analytic functions describing different types of material microstructure to experimental data in order to determine the shape, size and degree of ordering. SasView also includes tools for calculating scattering length densities, slit sizes, resolution, fringe thicknesses/d-spacings, the (Porod) invariant ("total scattering"), and distance distribution functions. More details can be found on the site http: //www.sasview.org/.

SRIM: Group of programs which calculate the stopping and range of ions (with an initial energy up to $2 \mathrm{GeV} / \mathrm{amu}$ ) into matter using a quantum mechanical treatment of ion-atom collisions (assuming a moving atom as an "ion", and all target atoms as "atoms"). TRIM (the Transport of Ions in Matter) is the most comprehensive program included. TRIM will accept complex targets made of compound materials with up to eight layers, each of different materials. It will calculate both the final 3D distribution of the ions and also all kinetic phenomena associated with the ion's energy loss: target damage, sputtering, ionization, and phonon production. More details can be found on the site http://srim.org/. 121 


\section{Introduction}

As it is well known since the founding experiment of Fermi and Zinn [45], the total external reflection of a collimated neutron beam on a flat surface is possible if the particles have an incident angle below a critical value $\gamma_{c}$ given by :

$$
\gamma_{c}=\lambda \sqrt{\frac{N b}{\pi}}
$$

where $\lambda$ is the neutron wavelength, $N$ is the mean number of scattering nuclei per unit volume and $b$ is the coherent scattering amplitude. The first measured value of $\gamma_{c}$ was done by Fermi and Marshall [46] using a single crystal of Calcium Fluoride as reflecting material.

As explained by Christ and Springer [28] and Maier-Leibnitz and Springer [67], this property can be used through beam guides for the transport of neutrons over tens of meters without losing intensity dramatically. The beam intensity not being linked any more with the square of the distance from the source.

Technically, neutron guides are made of four mirrors assembled together and installed permanently around a nuclear reactor or a spallation source. Notice from equation 1 that $\gamma_{c}$ depends on the material used for the mirror manufacturing, as well as the neutron wavelength. The interest of such apparatuses lie, therefore, in their wavelength filtering ability: a fine selection of the reflective material and tuning of the guide curvature will allow the rejection of the particles that cannot be used for experiments. Neutrons with an energy and an incident angle sufficiently low will be transported, while others will cross the mirror and penetrate in the guide substrate.

Neutron guides are widely used in neutron research facilities to transport moderated neutrons from their source to scientific experiments. They allow an increase in the number of instruments per source as well as the beam quality by filtering fast and epi-thermal neutrons from the beam. At their origin, neutron guides were made of natural Nickel, or its isotope ${ }^{58} \mathrm{Ni}$, coated on $2 \mathrm{~cm}$ thick borosilicate glass plates. They were assembled to have a rectangular cross section with dimensions around $3 \mathrm{~cm}$ by $20 \mathrm{~cm}$. Nowadays, glass thickness has been divided in half and the active surface became a $\mathrm{Ni} / \mathrm{Ti}$ multilayer, multiplying the critical angle by 2 or 3 . For example, the curvature of a guide dedicated to thermal neutrons is around $10 \mathrm{~km}$. The total length of a guided beamline at ILL is around $60 \mathrm{~m}$. Also, ongoing projects, such as the European Spallation Source, are studying the design of neutron guides measuring approximately $150 \mathrm{~m}$ long. 
The present Ph.D thesis was focused on the stability of such guides under irradiation for a long period of time. As will be explained in more detail, several research facilities around the world experienced incidents involving neutron guides, with the same results of having to shut off the beam for up to several months. These events ranged from the splintering of the first millimetre of glass under the mirror to the implosion of a whole guide element. It has been found that such incidents were linked to an excessive neutron dose absorbed by the mirror substrate, with the particles being mainly absorbed by the ${ }^{10} \mathrm{~B}$ isotopes of the borosilicate matrix. Originally, boron containing glass was selected for guide manufacturing specifically to absorb neutrons and act as a radiological shielding. The mechanical failure induced by those nuclear reactions had not been foreseen. The main aims of the study reported here are thus:

- To validate or reject specific glasses for their use as mirror substrates in guides to be deployed for the next decades, and

- To understand the origin of the differences in behaviour under irradiation of glasses having different chemical composition.

In other words, this work aims to study the stability of alkali-borosilicate glasses throughout their deep structural evolution when exposed to irradiation during a long period of time. For that, a whole set of material characterization experiments and several modelling tools have been employed. The work spanned from neutronic studies up to nano-, micro-, and macroscopic analysis of irradiated material using well established tools.

To summarize, the main thrust of this Ph.D. effort is to contribute towards a known and long-standing problem concerning neutron guides manufacturing in order to make them robust and radiation-resistant beyond the known current limits. Moreover, this issue is of great interest considering the very long beam lines that are under design for the future ESS. Indeed, with today's economic context, the main cost drivers of tomorrow's scientific instrument will surely regard the neutron guides, their installation, and upkeep throughout the years. 


\section{Chapter 1}

\section{Fundamentals and Problem setting}

This chapter will be focused on neutron guides science and technology and how they may evolve dramatically when exposed to an excessive radiation dose.

\subsection{Introduction to neutron reflection theory}

The neutron has played an important role as a probe for matter and materials for many decades through its interactions with the atomic nuclei. This being done by its nuclear and magnetic interaction properties. Even though it has a mass $\left(935.65 \mathrm{MeV} / \mathrm{c}^{2}\right)$, when talking about its propagation in a guide, it is handy to consider its wave nature. Its wavelength $\lambda$ is linked to its speed by:

$$
\lambda=\frac{h}{\gamma \cdot m_{0} \cdot v} .
$$

The quantity above is known as the thermal or de Broglie wavelength, and will play a fundamental role here. In fact, a good number of moderated neutron beam properties can be described using tools borrowed from Optics.

As a point of reference, at the ILL neutrons are delivered to scientific instruments having different energies which can be gathered into three different energy groups, namely cold, thermal, and hot. The table 1.1 gives equivalences for neutron energy.

To describe how a neutron wave interacts when reaching a substrate, we can consider an incident wave with a vector $\vec{k}$ propagated on the $(\mathrm{x}, \mathrm{y})$ plane and a substrate surface at " $x=0$ ". The incidence angle of the incoming wave to the substrate surface is $\phi$. The reflected and refracted waves are characterised by their wave vector $\mathbf{k}^{\prime}$ and $\mathbf{K}$, and their

\begin{tabular}{cccc}
\hline Neutron type & Cold & Thermal & Hot \\
\hline Temperature $(\mathrm{K})$ & 25 & 298 & 2400 \\
Energy $(\mathrm{meV})$ & 2.3 & 25 & 130 \\
Wavelength $(\lambda)$ & $>3$ & 1.8 & 0.8 \\
\hline
\end{tabular}

Table 1.1: Neutron Temperature - Energy - Wavelength equivalences 


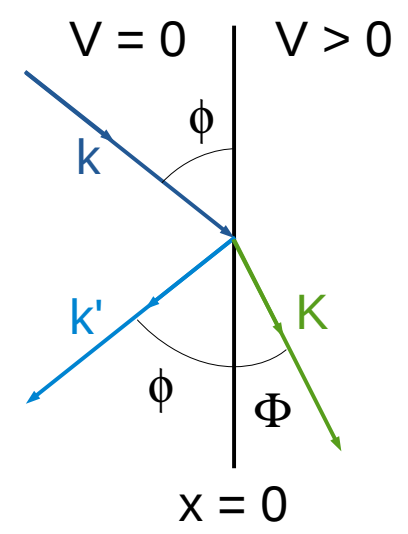

Figure 1.1: Reflection and refraction at a plane boundary

angle to the substrate $\phi$ and $\Phi$ respectively. The short range nuclear interaction between the neutron and the substrate generates an energy barrier

$$
V=\frac{2 \pi \hbar^{2}}{m} N b_{c}
$$

which is a volume averaged of the Fermi potential and is independent of the neutron energy. All those parameters are replotted in Fig.1.1. The resolution of Shrödinger's equation of the $\psi$ wave interaction with the surface gives such solution,

$$
\begin{array}{ll}
\text { for } x<0 & \psi(\mathbf{r})=a \cdot e^{i \mathbf{k r}}+a^{\prime} \cdot e^{i \mathbf{k}^{\prime} \mathbf{r}} \\
\text { for } x>0 & \psi(\mathbf{r})=A \cdot e^{i \mathbf{K r}}
\end{array}
$$

Before going further, remember the refraction index of a medium,

$$
n=\frac{K}{k}
$$

In our case,

$$
n^{2}=1-\frac{V}{E}=1-\xi
$$

The continuity of $\psi(\mathbf{r})$ and $\nabla \psi(\mathbf{r})$ at $x=0$ allows us to find the reflected and refracted amplitudes as a function of the incident and the refractive index on the $\mathrm{x}$ axis $n_{x}$,

$$
\begin{aligned}
& a^{\prime}=\frac{1-n_{x}}{1+n_{x}} a \\
& A=\frac{2}{1+n_{x}} A
\end{aligned}
$$

where,

$$
n_{x} \equiv \frac{K_{x}}{k_{x}}=\sqrt{1-\frac{\xi}{\sin (\phi)^{2}}}
$$

From those parameters it is possible to calculate the reflectivity of the substrate. It is defined as the amount of neutron reflected divided by the total number of incident 
neutrons,

$$
R=-\frac{j_{x}^{\prime}}{j_{x}}=\left|\frac{a^{\prime}}{a}\right|^{2}
$$

where $j_{x}$ is the neutron current density on the x-direction. This quantity is defined for a wave $\phi$ as

$$
\mathbf{J}=\operatorname{Re}\left\{\phi_{\mathbf{k}}(\mathbf{r})^{*} \phi_{\mathbf{k}}(\mathbf{r})\right\}
$$

By injecting Eq.1.6 and Eq.1.7 in Eq.1.8, it is possible to derive,

$$
R=\frac{|\xi|^{2}}{16 \sin ^{4}(\phi)}
$$

Numerical applications show that, for thermal neutrons, $\mathrm{R}$ is negligible unless being in grazing incidence conditions. For $\phi \rightarrow 0$,

$$
R=\left|\frac{1-\sqrt{1-(\sqrt{\xi} / \phi)^{2}}}{1+\sqrt{1-(\sqrt{\xi} / \phi)^{2}}}\right|^{2}
$$

It allows to define a critical incidence angle 98 above which reflectivity drops drastically,

$$
\gamma_{c}=\sqrt{\xi}=\lambda \sqrt{\frac{N b_{c}}{\pi}}
$$

From the above equation, one can calculate the critical angle for different materials and neutron wavelengths. In the case of neutron guides, it is interesting to calculate this value for glass, natural abundance nickel and nickel-58. Table 1.2 gives these values for neutrons with $10 \AA$ wavelength. Among all materials, nickel has one of the highest coherent scattering lengths [99]. Also, because of it is easy to coat on a flat surface, e.g. a glass plate, nickel was soon chosen as the reference material for the manufacturing of neutron mirrors. In order to improve the reflectivity, the first evolution of neutron mirrors has been to use ${ }^{58} \mathrm{Ni}$ isotope instead of natural Nickel. One can cite for instance the H5 guide system of the ILL, made with ${ }^{58} \mathrm{Ni}$ coating until its replacement in 2014 [42].

\begin{tabular}{cc}
\hline & $\gamma_{c}(\mathrm{rad})$ \\
\hline Glass & 0.011 \\
$\mathrm{Ni}$ & 0.017 \\
${ }^{58} \mathrm{Ni}$ & 0.020 \\
\hline
\end{tabular}

Table 1.2: Values of reflection critical angles for some materials

To improve the reflectivity, the technology of super-mirrors, using Bragg's law to reflect neutrons at higher incidence angle, were developed. The substrate does not change, but the coating turns into a $\mathrm{Ni} / \mathrm{Ti}$ multilayer [95]. While nickel is the active layer, titanium has been chosen to generate a high contrast of coherent scattering length with the nickel. For natural titanium, $b_{c}=-3.438$, which does not generate a critical incidence angle. The principle is that the thickness of the layers depends on their depth in the 
mirror. It can be directly derived from Bragg's Law :

$$
n \times \lambda=2 d \times \sin (\gamma)
$$

Where $\lambda$ is the neutron wavelength, $d$ is the layer thickness, $\mathrm{w} \gamma$ is the incidence angle on the mirror (bigger than $\gamma_{c}$ ) and $n$ is a natural number. The thickness decreases as the layer is deeper in the super mirror. Nowadays, the reflectivity of mirrors and their associated guides are characterized by their " $\mathrm{m}$ " value. It is defined, for a constant wavelength, by the critical angle of a given mirror divided by the critical angle of a regular nickel mirror. This gives ${ }^{58} \mathrm{Ni}$ an "m" value around 1.2. Thus, the critical angle of reflection can be defined for a super mirror by,

$$
\gamma_{c}=1.73 \times 10^{-3} \times m \times \lambda
$$

where the angle is given in radians and the wavelength in Angstroms. When studying the interaction of neutrons with mirrors, the Momentum Transfer is often considered :

$$
Q=\frac{4 \pi \times \sin (\theta)}{\lambda}
$$

where $2 \theta$ is the angle between the incident and the reflected beam. This variable can be used to plot the reflectivity curves for different values of $\mathrm{m}$ (see figure 1.2). The interest of doing so is that the reflectivity curve is no more dependant of the neutron wavelength. An analysis of those curves is mandatory to understand the reasons behind the losses in the transport of neutrons within a guide. Look at the " $m=2$ " curves :

For $\mathrm{Q}<0.02$, the reflection of neutrons occurs within the first nickel layer of the coating. The reflectivity is approximately equal to 1 . This value depends on the coating quality.

The region comprising $0.021<\mathrm{Q}<0.042$ can only be reached using super mirrors. The neutron enters into the Ni-Ti multilayer and is reflected if it fulfils the Bragg reflection conditions. However, due to absorption and incoherent scattering interactions, the reflectivity decreases [9]. As will be detailed further, in the case of a guide, neutrons belonging to this region will be lost on the whole guide length.

Finally, for $0.042<\mathrm{Q}$, the reflectivity drastically drops to 0 . For a guide, this part of the neutron spectrum is mostly rejected near the source, or at the first bounce of the particles on the guide mirrors.

The concepts exposed in this section (reflection, transmission and critical incidence angle) are key elements to understand why structural parts of neutron guides are exposed to irradiation and ageing.

\subsection{Neutron guide basic features - examples in large research facilities}

Traditionally, neutron guides are made of four mirrors assembled together. When the mirror substrate is glass, they are glued; when it is a metallic substrate, such as alu- 


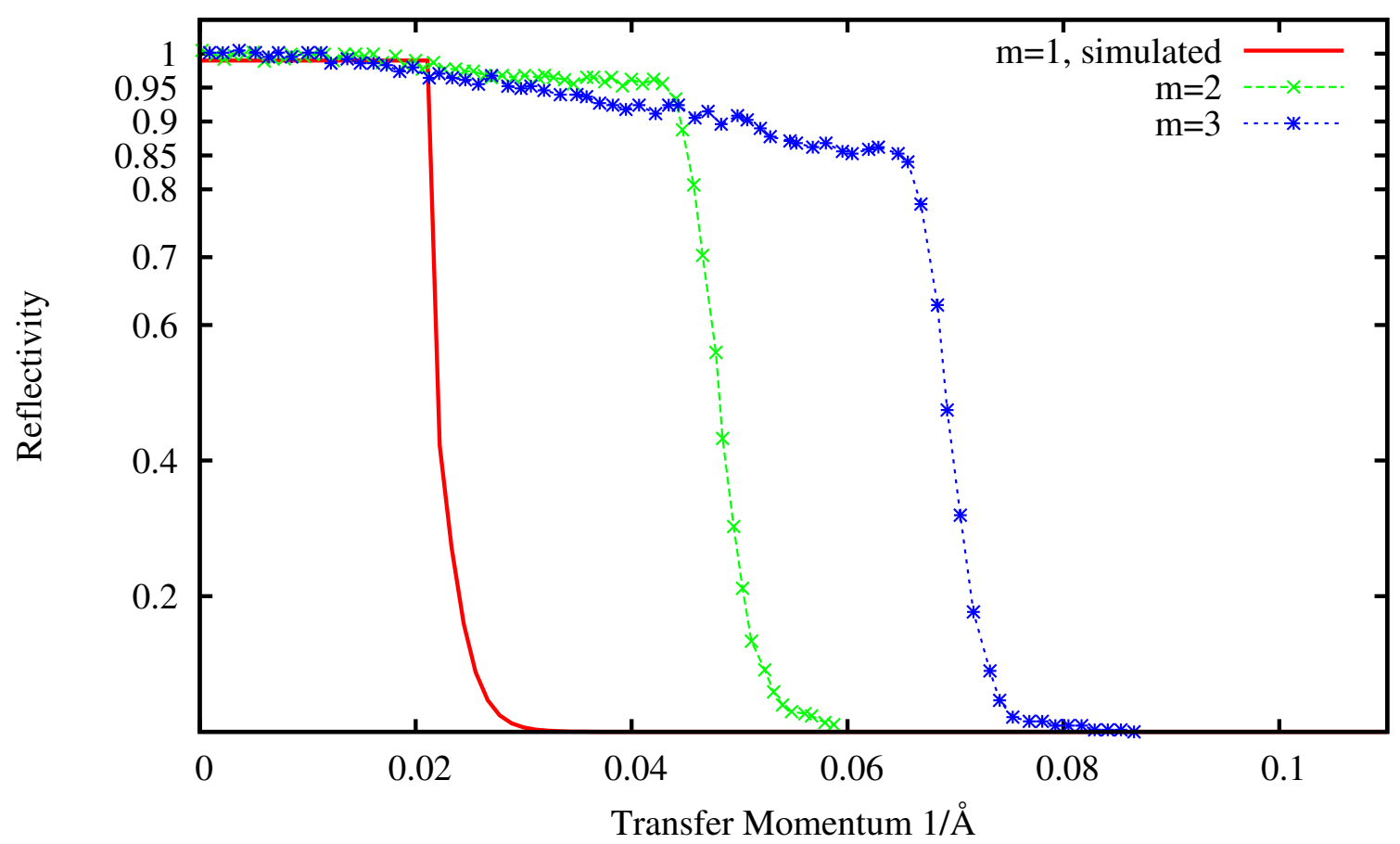

Figure 1.2: Reflectivity curves of mirror and super-mirrors. Simulations made following McStas formula [110] and measured values from Swiss Neutronics 106

minium, they are tightened with multiple screws. A whole beam line can reach up to 100 meters of length, and is composed of smaller elements that vary between 500 and 2500 $\mathrm{mm}$. The size depends on the technology used for the alignment of the elements. At the ILL, neutron guides come close to the neutron sources to collect the maximum flux and transport it to the instruments. The limit beyond which a neutron guide cannot be placed closer to the source is influenced by multiple factors, such as thermal heating of the glass due to various radiations of the source and the angular spectrum produced by the source that can be transported by the guide.

When transported in a guide, a neutron beam is defined by several parameters:

Intensity: usually expressed in neutrons per second per square centimetres : $\mathrm{n} / \mathrm{cm}^{2} / \mathrm{s}$

Wavelength spectrum: The wavelength at which the intensity is maximized mainly depends on the temperature of the neutron source (see table 1.1), although, as later explained, this can also be influenced by the guide characteristics.

Divergence: is the maximum $\mathrm{Q}$ value that can be propagated in the guide. It is usually expressed in the " $\mathrm{m}$ " value of the mirrors.

Spin: for scientific purposes, polarisers can be used to keep neutrons of the beam with the needed spin.

Even though the critical angle for thermal or cold neutrons is small, it is still possible to guide a neutron beam along a curved path. It is done by inserting an angle sensibly smaller than the critical angle of the wavelength of interest between the elementary guide 


\begin{tabular}{c|cc|c|c|c}
\hline & H113 & H24 & G6 & NL6 & CG3 \\
\hline Research center & ILL & ILL & LLB & FRMII & Ansto \\
Country & France & France \\
cold & thermal & France & Germany & $\begin{array}{c}\text { Australia } \\
\text { cold }\end{array}$ \\
Source energy & 90 & 30 & 25 & 60 & 50 \\
Width (mm) & 200 & 200 & 150 & 120 & 200 \\
Height (mm) & 2 & 1 & 2 & 2 & \\
m coating & 4000 & 12000 & & & \\
Curvature $(\mathrm{m})$ & 70 & 70 & & & \\
Length $(\mathrm{m})$ & \multicolumn{1}{|c|}{} & & & \\
\hline
\end{tabular}

Table 1.3: Some neutron guides' characteristics around the world

elements. The use of curved guides to supply neutron scattering experiments is driven by the following needs:

- To enable enough room to install instruments. For an experimental reactor, all the neutrons come from a relatively small core. For a spallation source, they are produced on the target/moderator/reflector system. Prior to the use of neutron guides, beams were transported to the experimental stations by the use of short beam or flight tubes, thus limiting the number of instruments that were possible to accommodate by the available surface surrounding the source. To address such issues, guides are installed to bring the neutrons well away from the building hosting the primary source and are then curved to be split into two or more, hence enabling the installation of more instruments. The H5 guide system of ILL is a good example of that principle. At the guide start, there would have been only room for a single instrument; whereas there are now eight instruments powered by three guides [18].

- To improve the beam quality. At the guide start, in addition to moderated neutrons, a beam contains a portion of epithermal, fast neutrons as well as gamma rays. These particles result in background noise for the scientific instruments. As mirrors do not reflect those particles, a curved guide will act as a filter for them. They will be transmitted through the mirror at their first bounce.

Only a few parameters in a guide influence the neutron transport and the output beam properties. They are listed in Table 1.3 and values are given for various guides from different facilities over the world.

The structural material commonly used for the manufacturing of neutron guides is glass. The main reason is that it can be easily polished to neutron optical quality. More into specifically, the waviness can be lowered below $10^{-4}$ radians (RMS value) [59]. In addition, some commercial glasses have boron rich compositions. The ${ }^{10} \mathrm{~B}$ isotope is a very good thermal neutron absorber $\left(\sigma_{(n, \alpha)}=3980\right.$ barns) and allows the glass to shield the guide environment from the non-reflected particles. Commercially available borosilicate

\footnotetext{
${ }^{1}$ The waviness is defined by the angle between the perpendicular to the surface at two different positions of a same reflective surface. To have $R M S$ of this value, one should compute the root-square of the averaged square waviness over the whole surface.
} 
glasses, such as Pyrex® or Bororofloat ${ }^{\circledR}$, exist in large quantities and are economical. Their boron oxide content suffices for their use in neutron guides and their manufacturing process gives them a surface state adapted for neutron reflectivity purposes. However, as will be explained later, their behaviour under high neutron flux is far from optimal.

One should mention the research efforts focused on finding a metallic substrate that could be used as guide walls instead of glass. So far, aluminium appears to be the best candidate; in fact, a surface roughness low enough for reflectivity purposes was achieved by recourse to the Canigen technique [112]. The main issue here, however, concerns cost. Metallic guides are, for the time being only, used for specific elements with high thermal constraints or geometric difficulties. As an additional drawback, the metal becomes highly activated under thermal neutron flux. To counter that effect, research on borated aluminium is being carried out. One should mention that NIST ordered a full aluminium guide in 2015.

\subsection{Beam wave propagation theory and losses in sub- strates}

To understand the degradation occurring in the glass mirror substrate, one needs to understand the mechanisms that cause neutrons to cross through the mirror and penetrate into the borosilicate material. A conceptual sketch of the theoretical tools required for such a purpose has been provided in a previous section. The losses due to the beam transport can be divided into two categories; namely, those which take place near the source and those occurring all along the beam line. A detailed description can be found in papers of Maier-Leibnitz and Springer, and later Bouton 24,67] gave detailed description of the theory and application of the neutron transport in beam tubes. Here, we intend to give some keys to understand the entire mechanism.

\subsubsection{Liouville's Hamiltonian theorem}

Neutron transport is, in general, lead by Liouville's Hamiltonian theorem [62]. It states that, for a Hamiltonian dynamic system, the distribution function is constant along any trajectory in phase space. As the reflections of neutrons over the guide walls do not dissipate energy, their transport can be considered as a closed system and the Liouville's theorem applies. It is not strictly true because of the gravitational force, however its effects on thermal and cold neutrons in a neutron guide are often disregarded.

Considering the neutron beam transport in a guide, the theorem can be recast into an alternative form that states that one cannot compress the phase space along the guide. If one considers a guide with a given width, shrinking by a factor of two along the way, the divergence of the beam must be multiplied by a factor of two to keep all the neutrons. In reality, some neutrons will be transmitted through the mirrors and lost in the focusing section. Another way to view this theorem is to realize that one cannot go above the primary source brilliance $\left(\mathrm{n} / \mathrm{cm}^{2} / \mathrm{str} / \mathrm{s}\right)$ by optical techniques. 


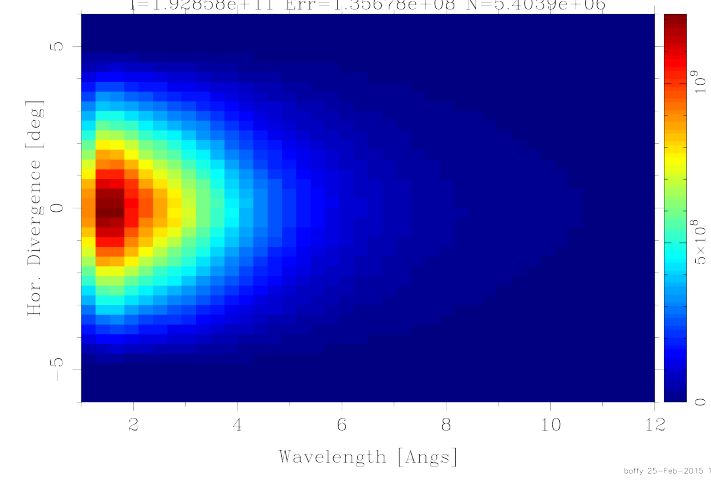

(a) Wavelength and horizontal divergence

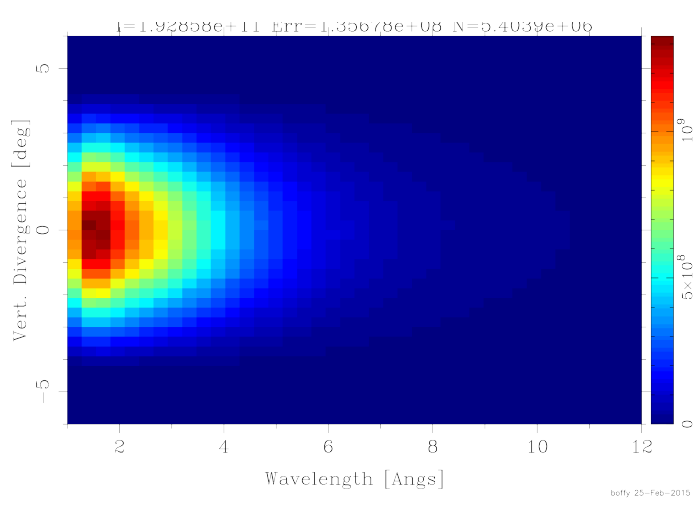

(b) Wavelength and vertical divergence

Figure 1.3: Density plots of the beam intensity at the entrance of the H5 guide at ILL. McStas simulations.

\subsubsection{Typical neutron source and flux at a guide entrance}

If we take the example of the Horizontal Cold Source (HCS) at ILL, its shape is spherical with a diameter around $200 \mathrm{~mm}$. It feeds the newly renovated H5 guide system, which starts at a distance of 2.155 meters from the source center. Then, if one takes a neutron coming from the HCS edge and passing at the center of the guide opening, its divergence can be estimated to $2.7^{\circ}$. If the mirrors are set at " $\mathrm{m}=3$ " on the first meters, no neutron below $9 \AA$ can be reflected with such an incidence angle onto a super-mirror. The result is that a lot of neutrons will be lost along the first meters of the H5 guides. Nevertheless, the advantage of such a large source is that it feeds several beam lines positioned alongside each other. Figures $1.3 \mathrm{a}-\mathrm{b}$ are contour plots of the neutron beam at the entrance of the H5 guide; the coordinates are wavelength versus horizontal or vertical divergence, respectively. The simulation results shown are here based on the new H5 geometry, with the Monte-Carlo source scaled on gold foil measurements done in the different H5 guides. The McStas [60, 109 software has been used to compute those results.

If one applies equation 1.14 with an " $\mathrm{m}=3$ " guide, we can see that most of the neutrons will not be reflected. This forces researchers to change the guides near the source often. Currently at ILL, in-pile elements are replaced every 10 years. However, as it will be detailed further, Pyrex® that was installed in-pile broke unexpectedly 4 years after commissioning in the 1970s.

\subsubsection{Effects of curved sections on neutron beam properties}

As explained by Maier-Leibnitz [67], a curved neutron guide used to transport moderated neutrons can also be used to filter a part of the beam spectrum. The increase of the incidence angles on the mirrors outside of the curvature will bring particles outside of the reflectivity domain. Three guide parameters will affect the beam properties :

- The curvature radius, usually noted as $\rho$.

- The guide dimension in the curved direction. This is measured by either, its width or height, depending on whether it is bent horizontally or vertically. 
- The m-value of the mirrors.

If one considers a straight guide illuminated by an infinitely large source, far from the entrance, the divergence cone that can be accepted by the guide is directly linked to the $\mathrm{m}$-value of the mirrors. Neutrons can be found at any point in the guide cross section as long as their incidence angle to the guide walls is below $\gamma_{c}$.

This is not the case in a curved guide. If we consider a beam bent horizontally, the angular cone $\theta_{c}$ that can be transported depends on the distance to the concave wall:

$$
\theta_{c}=\sqrt{\gamma_{c}^{2}-\frac{2 x}{\rho}}
$$

Where $\gamma_{c}$ and $x$ represent the critical incidence angle and the distance to the concave wall, respectively. From that formula, one can see that the closer you are to the inside the curvature, the more the accepted cone is reduced. Keep in mind from the Liouville theorem, $\mathrm{p} 9$, that the neutrons at the entrance of the curved section above the accepted cone will be filtered. That is to say those neutrons will be transmitted through the mirror toward the substrate at the first bounce. From that explanation, one can see that the mirror substrates outside the bend are usually more exposed to neutron irradiation. Using $\theta_{c}$ from the formula 1.16 , we can be calculate what is called the guide filling:

$$
f(x)=\frac{\theta_{c}}{\gamma_{c}}=\sqrt{1-\frac{2 x}{\rho \cdot \gamma_{c}^{2}}}
$$

It represents the ratio of divergence transported in a bent guide compared to a straight one as a function of the distance from the concave wall. The calculation of the average value over the entire guide width gives the global angular transmission of the guide for a fixed wavelength,

$$
T^{\text {angular }}=\int_{0}^{X} \sqrt{1-\frac{2 x}{\rho \gamma_{c}^{2}}} \cdot d x
$$

where $w$ is the guide width in the curved direction. For $\frac{\gamma_{c}^{2} \rho}{2}>w, X=w$ else $X=\frac{\gamma_{c}^{2} \rho}{2}$. Figure 1.4 displays angular filling curves (eq. 1.17) of three different wavelengths for a typical cold guide: $\rho=4000 \mathrm{~m}, w=30 \mathrm{mmm}$ and $m=1$ mirror. Each curve is characteristic of a special transport regime:

$3 \AA$ : Neutrons can be found at every position in the guide. The neutrons with the highest angular divergence are transported by successive bounces from one wall to the other, called "zig-zag" reflections. The difference from the curve to an angular filling equal to 1 represents the neutrons lost at their first bounce in the concave wall. For that wavelength, $T^{\text {angular }}=84 \%$.

$2.24 \AA$ : It is the characteristic wavelength of that guide, often written $\lambda^{*}$. Below it, neutrons no longer touch the convex wall. This wavelength is often used when dimensioning a new guide for its typical transmission coefficient: $T^{\text {angular }}=2 / 3$ for $\lambda^{*}$ and $93 \%$ for $2 \lambda^{*}$. 


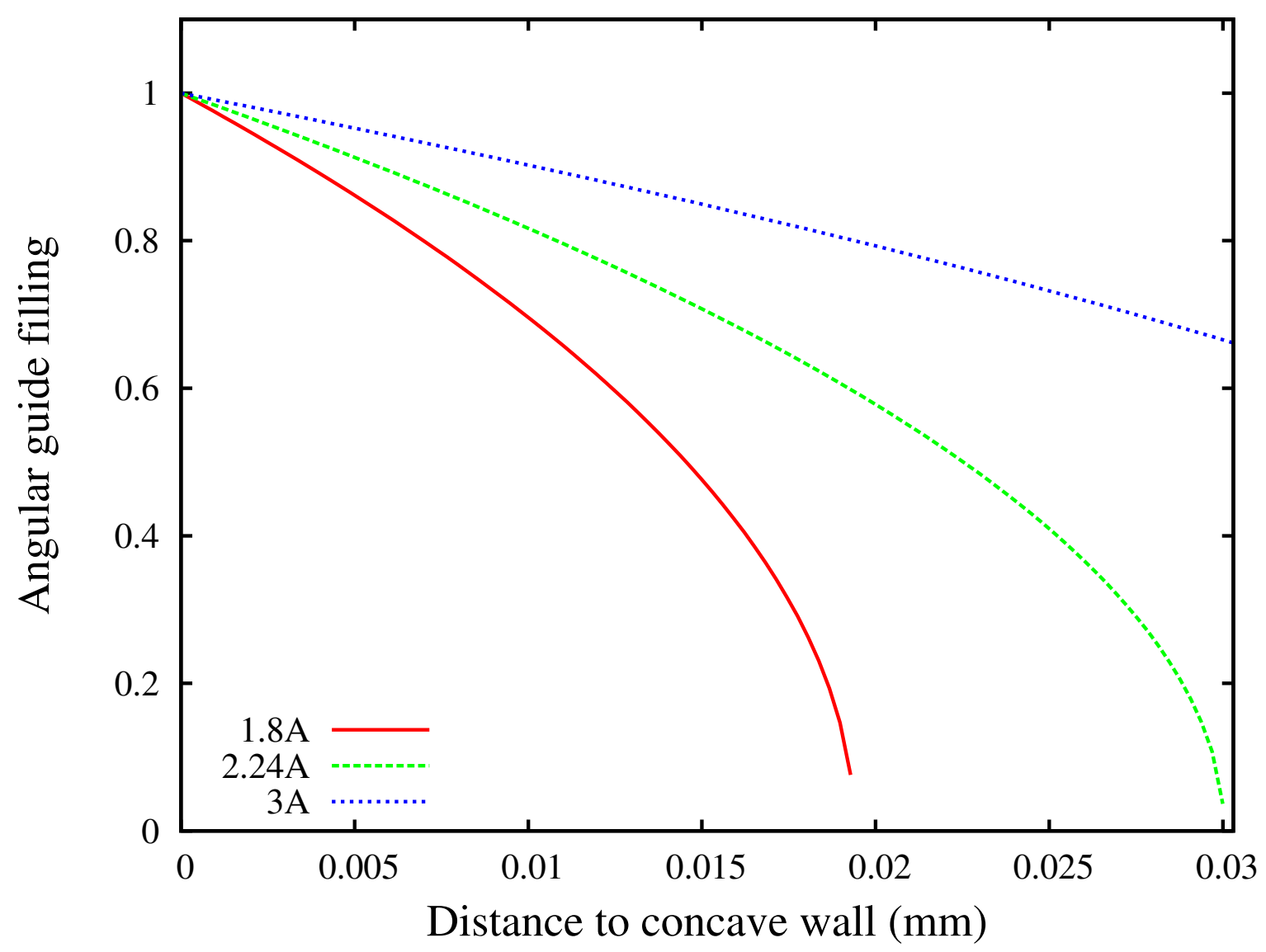

Figure 1.4: Guide angular filling as a distance to the concave wall, calculated from formula 1.17, $\rho=4000 \mathrm{~m}, a=30 \mathrm{~mm}$ and $m=1$ mirror

$1.8 \AA$ : It corresponds to thermal neutrons; they are only transported by bounces on the concave wall, called "garland" reflections. As previously mentioned, the characteristics of the guide are of a cold type. The reason is that the transmission is only $43 \%$ for $1.8 \AA$ neutrons, which is too low to be accepted to feed scientific instruments using thermal neutrons.

The characteristic wavelength, mentioned above, can be directly calculated through the formula,

$$
\lambda^{*}=\frac{\sqrt{\frac{2 w}{\rho}}}{1.73 \times 10^{-3} \times m}
$$

The wavelength filtering phenomenon forbids curving a guide too much. This can lead to being unable to access any space needed to install an experiment. From an initial source point feeding several guides, instruments are usually installed in a narrow fan-like shape. It should be mentioned that this limitation can be turned into an advantage. A fine tuning of $\lambda^{*}$ will allow to reject the neutrons that are not used by the scientific instruments. 


\begin{tabular}{c|c|c|c}
\hline & H5 & H52 & H522 \\
\hline Width $(\mathrm{mm})$ & 170 & 60 growing to 120 & 60 \\
Height $(\mathrm{mm})$ & 120 & 120 growing to 240 & 115 \\
Horizontal curvature radius $(\mathrm{m})$ & - & 4000 & 4000 \\
Super mirror coating (m-value) & 4 & 3 & 2 \\
Approximative length (m) & 1 & 18 & 35 \\
\hline
\end{tabular}

Table 1.4: H522 guide properties, from the H5 guide start

\subsubsection{Losses along a beam line - the example of H522}

To illustrate the loss of neutrons along a beam line, we can take the example of the newly renovated H522 ILL guide. It has been simulated using the McStas ray tracing software. The simulated geometry of the guide was taken from the design office plans. The shape of the neutron source spectrum was adjusted according to MCNP simulations of the ILL reactor, and the global intensity was scaled on gold foil measurements made at different positions along the guide. H522 comes from the subdivision of the H52 guide, itself being a sub part of the wide $(170 \mathrm{~mm}) \mathrm{H} 5$ beam. The H522 geometry varies greatly from the entrance to the end. However, Table 1.4 only reports the main properties of the beam from the entrance to the C9 housing. The simulation stopped at that point because, at the time of the calculation, the guide was not installed further.

Figure 1.5 shows the flux intensity simulation result as a function of the distance from the HCS. Because of the simulation accuracy, y-errorbars are almost invisible on that plot. One can clearly see that the nearer the guide is to the source, the more it is exposed to radiation. However as it will be illustrated later, some borosilicate glasses broke even though they were installed far from the moderator. The two points around $21 \mathrm{~m}$ are remarkable. One can see that the flux increase from $8.0 \times 10^{9} \mathrm{n} / \mathrm{cm}^{2} / \mathrm{s}$ to $8.7 \times 10^{9} \mathrm{n} / \mathrm{cm}^{2} / \mathrm{s}$. This is due to the $\mathrm{H} 52 / \mathrm{H} 522$ transition. As explained in subsection 1.3 .3 . curving a guide will induce a drop of flux on the inner side of the bend. As the $\mathrm{H} 522$ entrance is located on the outer side of $\mathrm{H} 52$ bend, it collects the most intense part of the H52 beam. The number of neutrons lost along the beam line could be calculated by deriving the curve of Fig. 1.5 .

\subsection{Improving the design of neutron guides}

Any improvement on the design of a given object, once the sought functional specification has been set, starts from its basic parameters such as its material components, shape, dimensions, weight, etc. In the very specific case of a neutron delivery system, such as a guide, the development of a new design comprises a good number of parameters including the shape of the mirrors, the beam size, the shape (and curvature) of the beam line, the mirror coating and substrate materials, which protective housing to choose, the selection of the alignment system, and the on-site installation set up, to cite a few. The initial selection of tasks to be comprised within this current Ph.D. thesis was to focus on issues pertaining to beam line geometry as well as the behaviour of the mirror substrate. Initial steps were taken to advance in both directions. However, after some months of 


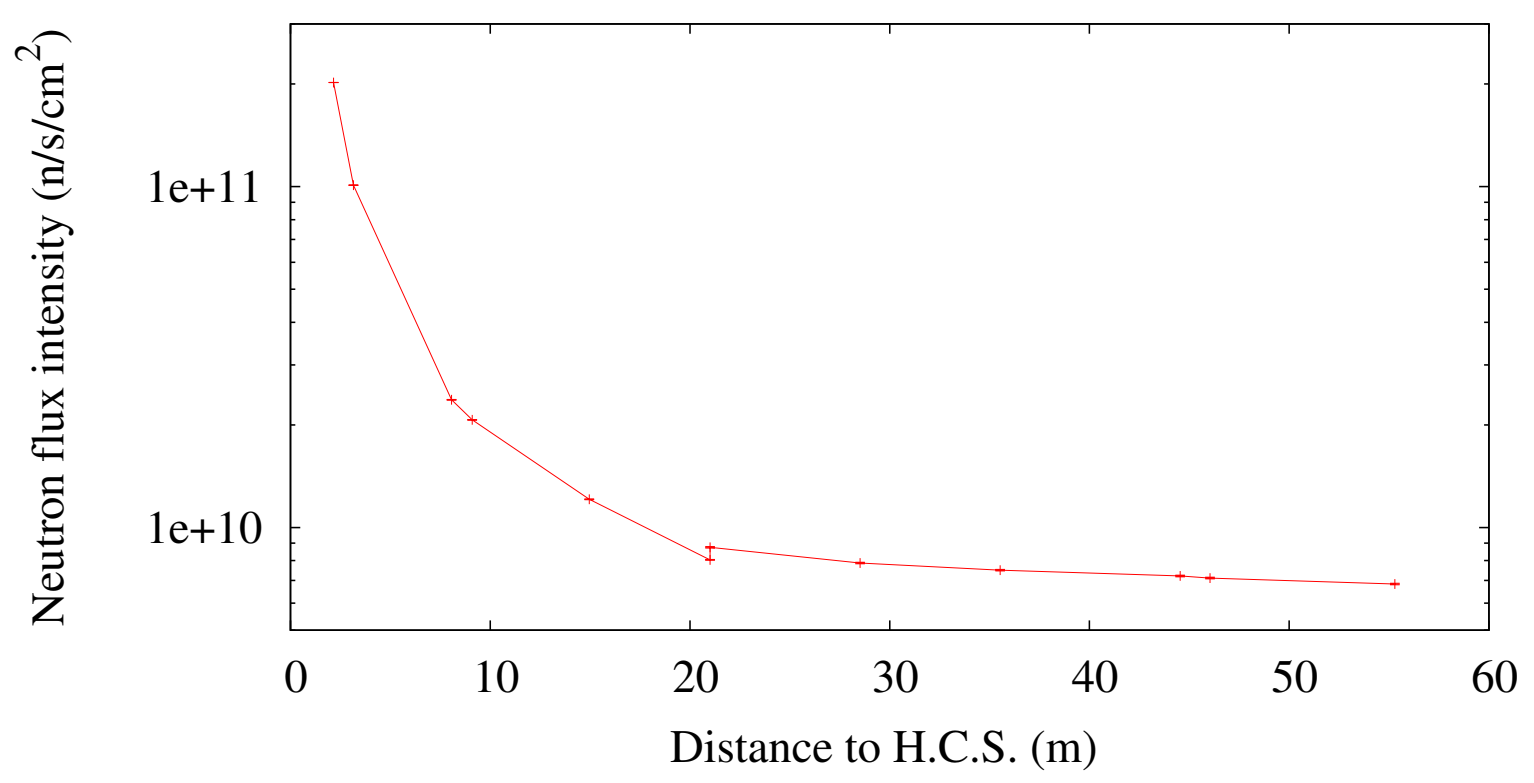

Figure 1.5: Flux in H522 neutron guide (ILL), McStas simulation with intensity scaled on gold foil measurements

work on both topics, we came to the realization that the fields to cover were far too broad. After carrying out some design studies focused onto neutron transport issues for new guide systems, we decided to concentrate our efforts into just one of such issues: the behaviour of mirror substrate under irradiation, and more specifically, the response to radiation when the material in question is a borosilicate glass. Such a decision was made on the grounds of two main considerations. First of all, the issue of glass ageing under irradiation has been known amongst neutron science communities for decades and still needs to be solved. As will be explained in the next section, some progress has been made on the understanding of the underlying reasons behind the splintering of some guide elements, but no clear quantitative understanding of the phenomena has been achieved. The second reason concerns the challenge that the radiation stability issue represents for instruments foreseen within the ongoing European Spallation Source project, especially if one considers the high fluxes the guides are supposed to transport to instruments when the machine will be running at full power, and their record length proposed for some of the beam lines. On such grounds, one would expect that unless such issues are solved, the ageing incidents noted in today's sources will perpetuate at ESS and will seriously compromise the installation reliability.

In light of the considerations written above, and in order to deliver a homogeneous document, most of the ensuing chapters deal with the understanding of borosilicate behaviour under thermal neutron irradiation. But, as explained earlier, a non-negligible amount of work was done on the development of innovative guide geometries. Part of the design efforts on new neutron delivery systems is summarized in appendix A. The efforts reported there were focused on the shaping of preliminary geometries for thermal and cold neutron guides. Such tasks were included within the framework of the Institute-LaueLangevin Endurance program, whose main aim was to refurbish four older beam lines, H15, H16, H23, and H24, in order to provide a more intense flux to existing instruments 
as well as to make room to accommodate new instrument developments.

\subsection{Guide ageing and reported incidents}

The fact that neutron guides are subjected to possible failure has been an issue well known by neutron science institutes for a long time. Although guide technologies have substantially improved over the last few decades and better materials are now employed for their construction, from time to time incidents continue to happen. A brief description of some of these incidents is below.

\subsubsection{ILL}

H25 - 1978

The first documented incident involving critical failure of a neutron guide happened at the ILL at the end of the 1970s [21]. It involved the $\mathrm{H} 2$ thermal guides that were manufactured using Pyrex ${ }^{\circledR}$ glass. The guide was commissioned in May 1972 and the technical services detected a drop in its flux merely four years later. The worst case registered at the installation concerned the H25, guide which had lost $35 \%$ of its flux. An inspection of the H25 in-pile part showed that some mirror walls had collapsed, see figure 1.6. The other $\mathrm{H} 2$ guides did not show such striking failures, but their mirrors showed significant cracks. After a macroscopic irradiation study of different guide support glasses, old guides were removed and replaced by new ones made of A8866 borosilicate glass (Corning-Sovirel). The physical phenomenon responsible for the ageing of the glass substate was ascribed to moderated neutrons transmitted through the mirrors, which ended up interacting within the glass substrate. This is called grazing incidence irradiation.

\section{H113 - 2005}

The H113 guide was commissioned in 1999 and was the first ballistic guide of the ILL. This type of beam line has a shape which is specially designed to reduce the number of neutron reflections from the source to the instrument, thus improving the transmitted flux. The technique is based upon a linear widening of the guide in its first meters, followed by narrowing as it approaches the experimental stations. Its main advantage stems from the fact that it lowers the beam divergence and reduces the number of neutron bounces on the guide walls. In the same vein, let us also mention the existence of typical guide geometries developed to improve the beam transmission, which are referred to as elliptic or parabolic $[56,58$ depending upon the shape of the guide walls.

The section of H113 showing the most serious damage used Borofloat@ as its structural glass. A visual observation inside the guide could easily detect that its reflectivity was deteriorated. The figure $1.7 \mathrm{~b}$ shows the supermirror layer peeling off from the guide walls. In comparison to the 1978 episode, this guide was irradiated at grazing incidence by neutron beams with a lower energy as the guide was fed by a cold source. However, as it will be explained later, the damage induced in the glass is fairly similar for thermal and cold neutrons. In 2006, Borofloat broken elements were replaced with float glass on the first 3.5 meters, and then with Borkron. 

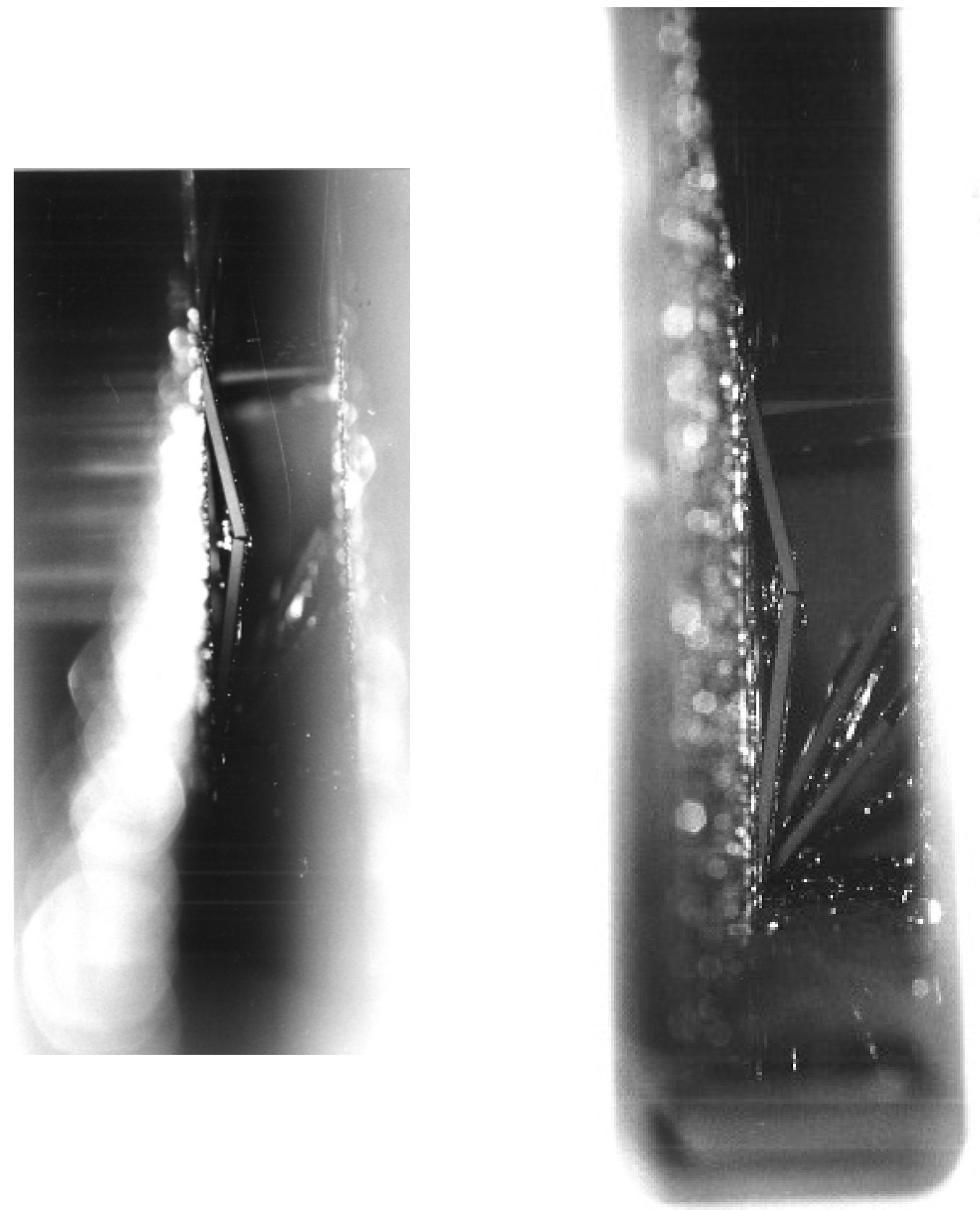

Figure 1.6: Photos of the H25 guide, in-pile elements 
The incident commented on here refers to damage to a frontal neutron irradiation. In March 2005, the H17 beam line imploded near a guide cut ${ }^{2}$. The glass used to manufacture the mirror was Borkron, and the guide was not placed within an evacuated housing. As the transport of neutrons is done in a vacuum, atmospheric pressure was generating stresses on the guide walls. This impressive incident, see figure $1.7 \mathrm{a}$, was caused by a combination of two deleterious phenomena:

The unshielded guide front face. After a guide cut, the slice of the downstream guide is exposed to frontal neutron beams. Currently, to account for this risk, these guide sections are protected by some neutron absorber layer, such as sintered $\mathrm{B}_{4} \mathrm{C}$, Cadmium, etc., but back then it was not the practice.

The on-girder installation. As explained before, the guide was not installed in an evacuated housing. The stresses provoked by the neutron irradiation were enhanced by the atmospheric pressure. Finally, as soon as the first micro-cracks appeared due to the irradiation ageing, they were amplified by the static pressure on the mirror walls and lead to the implosion of the guide elements.

This incident made the technical services realize that such guide failure could lead to the transport of relatively large guide debris at speeds of a few tens of meters per second, thus able to cause some significant damage on nuclear safety elements.

\subsubsection{Other research institutes}

Mechanical failure of neutron guides due to radiation ageing has occurred all over the world on similar substrates as those used at ILL. Institutes such as LLB, NIST [55], ANSTO [89], or FRMII [61] reported several incidents throughout the years. The ILL technical division that discovered Borofloat and Borkron weaknesses to irradiation in 2005 sadly observed the same scenario happening in other facilities some years later. Table 1.5 gives some details on guide breakdowns that happened at the research centres mentioned earlier.

\subsubsection{Brief comments on the reported incident conditions}

From the different incidents mentioned here, we can isolate three parameters that influence the failure phenomenon and concern:

- The glass type, namely, Pyrex, Borofloat, Borkron etc.

- Whether the guide was under atmospheric pressure or in evacuated environments, and

- The angle of neutron incidence, that is, whether this concerns frontal or grazing conditions.

\footnotetext{
${ }^{2}$ Guide cuts are places where there is no neutron guide on a distance that can go up to $300 \mathrm{~mm}$. Such sections are built to allow installation of mechanical elements used to either control the beam delivery (shutters), or to feed a scientific beam line (i.e. illuminating a monochromator).
} 


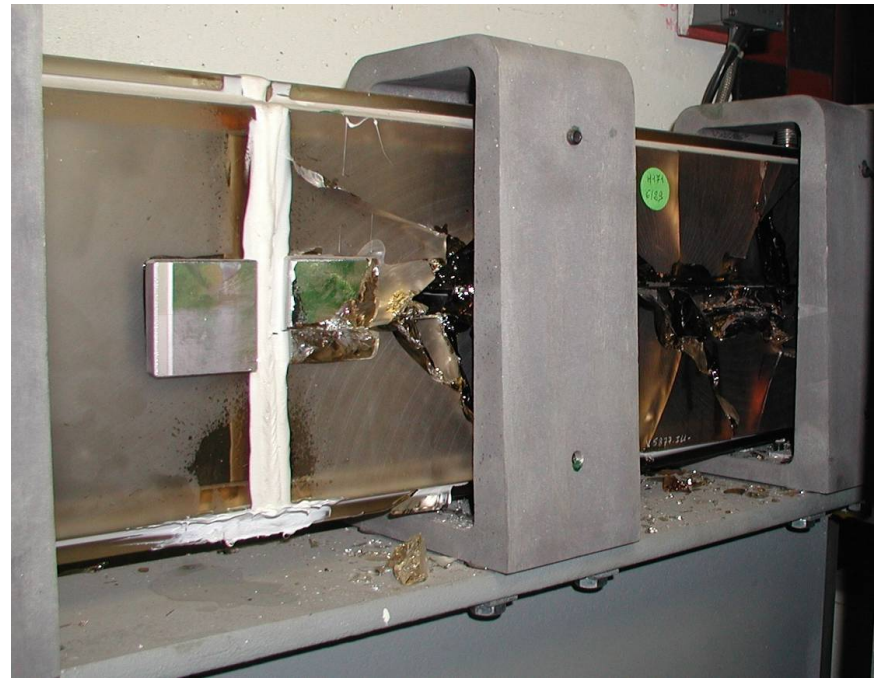

(a) H17 guide after implosion

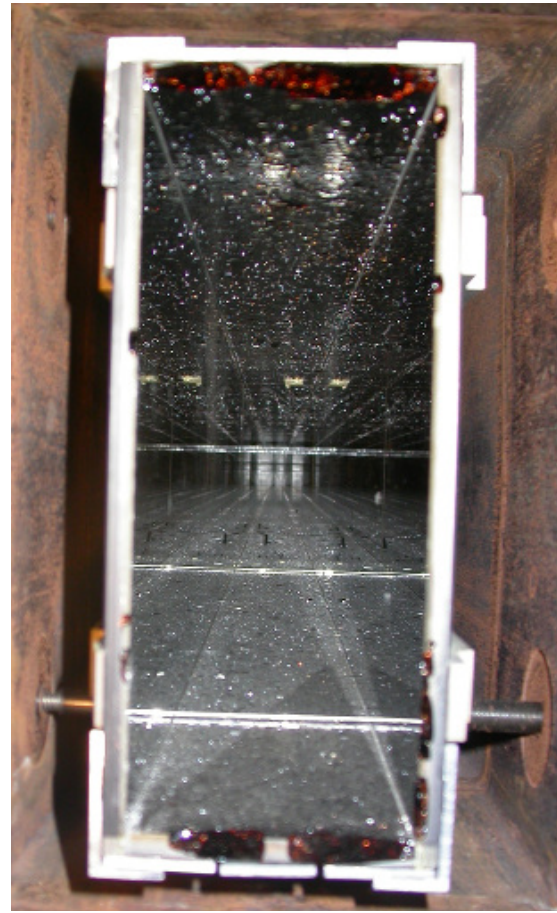

(b) H113 guide, peeling of the mirrors

Figure 1.7: Guide breakups due to over irradiation in 2005

\begin{tabular}{c|c|c|c|c}
\hline Beam line & G6 & NG6 & CG3 & NL-5 and NL-6 \\
\hline Institute & LLB & NIST & ANSTO & FRMII \\
Country & France & USA & Australia & Germany \\
Glass & Borofloat & Borkron & Borofloat & Borofloat \\
In evacuated housing & no & no & yes & no \\
Commissioning & 2005 & 1999 & 2007 & - \\
\hline Irradiation conditions & Frontal & Frontal & Grazing incidence & Grazing incidence \\
Incident conditions & Implosion & Implosion & Mirror peeling off & Implosion \\
Degradation detection & 2007 & 2005 & 2011 & 2012 \\
\hline
\end{tabular}

Table 1.5: Some guide destruction caused by over irradiation around the world 
From the three listed characteristics, we can make some preliminary remarks on the role of on girder installation. From what has been reported, it appears that the guides that are not installed within a housing tend to implode, whereas those protected from atmospheric pressure see their glass plate splinter right under the mirror.

Between the different glasses and accounts made of the incidents over the years, Borkron was described as being more radiation resistant than Borofloat. Some details pertaining chemical and manufacturing similarities between Pyrex and Borofloat will be discussed later on.

Finally, the incidence conditions only affect the intensity of the exposition. Grazing incidence happens when neutrons are transmitted through the mirrors and penetrate within the substrate, although the fraction of such neutrons from the original beam is usually small. Frontal irradiation occurs after a guide cut, if the beam divergence or the cut length are large, the flux on the guide slice may become comparable to that is actually transported. In both cases, the irradiated thickness is small compared to the rest of the substrate. 


\section{Chapter 2}

\section{Radiation effects in glass structure}

\subsection{What is glass?}

As it has been established in the previous chapter, neutron guide failures induced by radiation are associated with changes occurring within the glass substrate onto which the neutron reflecting material is deposited. Within the current section, we will introduce some concepts regarding the physics of glassy matter, which we will make use of in further sections.

The answer to the question, "What is glass?" may seem obvious for most people because of the use of such materials in everyday life in applications that go from kitchenware to house construction and smart-phone tactile screens. However, it becomes more difficult to find an answer that satisfies most of the community of specialists on the field of glassy matter. Such difficulty stems from the fact that glassiness regards phenomena exhibited by a wide variety of physical systems, well apart from windows or kitchen glasses. In fact, perhaps the most widely accepted definition that can be given for a glass is that it regards any physical system that has become non-ergodic by the effect of a continuous slowing down of one or more of its degrees of freedom. Within such a definition there is ample room to accommodate the glassy behaviour of systems as diverse as spin-glasses, orientational- and vortex-glasses, as well as some biological and granular systems.

The sort of glasses we are interested in are usually referred to as structural glasses, and these are best defined as amorphous solids which are capable of passing continuously from the equilibrium liquid into the supercooled liquid state, and finally into a solid-like state. This process is usually accompanied by an abrupt change in its heat capacity [16]. Put into different words, such glasses are systems which have some degree of freedom, usually related to liquid flow motion, which fluctuate at a rate strongly dependent on temperature and/or pressure. At a given value of temperature or pressure, such fluctuation becomes so slow that it becomes frozen at a laboratory scale. Such specific values of the thermodynamic fields that mark the transition into solid-like states are referred to as the glass-transition temperature $\left(T_{g}\right)$ or pressure $\left(P_{g}\right)$, respectively. The arrest of such a degree of freedom when say, cooling a liquid, means that the state of internal equilibrium characterizing the liquid at equilibrium is lost. The solid glass represents a state where the ergodic hypothesis of classical statistical mechanics breaks down.

Crossing from the liquid state into a solid glass takes place over a range of temperatures usually referred to as the glass-transformation range, and the extent of such a range of 
temperatures depends upon the cooling rate [16. This means that there is not a sharp thermodynamic transition, such as those characteristic of phase transformations within crystals, and therefore the definition of $T_{g}$ becomes somewhat arbitrary, although it has been defined as the temperature at which the liquid viscosity reaches a value of $10^{12} \mathrm{~Pa}$.s. Therefore, a solid glass of a given composition may show somewhat different properties depending upon its cooling rate production.

From the above paragraphs, it becomes clear that vitrification of a liquid constitutes a kinetic process. It can be described in simplified form using a variety of theoretical tools, which at the thermodynamic level, mostly comprise concepts from the thermodynamic of irreversible processes. In turn, at a microscopic level, advances within variants of the Kinetic Theory within Statistical Mechanics (i.e., derivations from the Boltzmann Transport Equation) have provided very relevant insights into the physics of the glassy transformation during the past few decades. These insights point towards the presence of a further critical temperature $T_{c}$ which, according to the followers of such concepts, constitutes the physically sound parameter and represents a temperature where motions are arrested and is thus independent of the cooling rate.

The next paragraphs provide relevant concepts for he description of the structure of a structural glass or amorphous solid.

\section{Description of a glass structure}

Contrary to the case of ordered solids or crystals, where their structure can accurately be specified in terms of Bragg planes, the description of the structure of an amorphous solid (or even a liquid) can only be attempted in terms of average quantities. Here, we follow a simple derivation of the basic tool used to describe a glass structure, which can be related to experimentally accessible quantities such as the pair distribution function $g(r)$. To such an avail, let's consider a function only dependent on a radial distance, say $m(r)$, which can take a value of 1 if $r$ lies within a given volume $V_{A}$ and zero otherwise. The number of particles enclosed within such a volume is simply,

$$
N_{A}=\sum_{i}^{N} m\left(r_{i}\right)
$$

where $N$ stands for the number of particles in the whole system. LEt us define the configurational distribution function of such a $\mathrm{N}$-particle system as $F_{N} N$, and is normalized such as $\int \ldots \int F_{N} N d N=1$. Such distribution function represents all the possible ways in which the particles are distributed. Here, $N$ represents the set of particle coordinates at a given instant $r_{1}, r_{2} \ldots, r_{N}$. Integration of $F_{N} N$ over $N-n$ particles yields the reduced, n-body distribution function,

$$
F_{n} n=\frac{N !}{(N / n) !} \int \ldots \int F_{N} N d N-n
$$

From here, it can be shown that the one-body density function just becomes,

$$
F_{1}(r)=N \int \ldots \int F_{N} N d N-1=\rho_{1}
$$


that is, a constant giving the system number density. In turn, the two body function becomes,

$$
F_{2}\left(r_{1}, r_{2}\right)=N(N-1) \int \ldots \int F_{N} N d N-2=\rho_{1}^{2} g(r)
$$

where $r=r_{2}-r_{1}$ and $g(r)$ stands for the probability that a particle is found within a volume element $d r$ surrounding $r$ when there is a particle at the origin $r=0$. The average number of particles contained within a given volume is thus,

$$
<N_{A}>=\sum_{i}^{N} \int \ldots \int m\left(r_{i}\right)
$$

and in a similar fashion, the average-squared number of particles can be written as

$$
<N_{A}^{2}>=n V_{A}+\sum \sum_{i \neq j}^{N} \int \ldots \int_{V} m\left(r_{i}\right) m\left(r_{j}\right) F N d N
$$

which can be expressed as,

$$
<N_{A}^{2}>=n V_{A}+n^{2} \int \ldots \int_{V_{A}} g\left(r_{1}, r_{2}\right) d r_{1} d r_{2}
$$

As shown above, $g\left(r_{1}, r_{2}\right)$ only depends on the difference $r_{1}-r_{2}=r$ and becomes unity for values of $r$ above a certain range, which is material dependent. As we shall see in the experiment sections, $g(r)$ may be determined from measurement, since the interference part $i(Q)$ of the diffracted intensity of a neutron beam becomes,

$$
i(Q)=\int_{0}^{\infty} D(r) \sin (Q r) d r
$$

where $Q$ stands for the momentum transfer and

$$
D(r)=\sum_{i} \sum_{j} b_{i} b_{j}[4 \pi \rho r(g(r)-1)]
$$

where $b_{i}$ stands for the bound nucleus scattering length. The relationship of the quantities just written above to experimentally accessible magnitudes is straightforward. In fact, the interference function $i(Q)$ given in Eqn 2.8 is defined as,

$$
i(Q)=\frac{d \sigma}{d \Omega}-\sum_{i} \bar{b}_{i}^{2}=\sum_{i} \sum_{j} \bar{b}_{i} \bar{b}_{j} S_{i j}(Q)
$$

where $d \sigma / d \Omega$ stands for the single-differential neutron scattering cross-section, accessible through diffraction measurements, which in turn comprises,

$$
\frac{d \sigma}{d \Omega}=\sum_{i} \overline{b_{i}^{2}}+\sum_{i} \sum_{j} \bar{b}_{i} \bar{b}_{j} S_{i j}(Q)
$$

That is a first term usually referred to as "self" which groups all the intensity contributions arising from spin-incoherent effects as well as those of coherent nature not giving rise to 
interference effects, and a second term which contains all the structural information. The bar over the scattering lengths $b_{i}$ indicates an average over nuclei on a given site $i$. The partial structure factors $S_{i j}(Q)$ thus contain all the structural information regarding structural correlations between atoms of type $i$ and $j$. A single measurement on a glass sample will provide an average quantity $S(Q)$, where all correlations are weighted by the individual nuclear scattering lengths. The neutron diffraction technique can, however, be employed in cases where isotopes of a given element do show significant differences in scattering lengths, thus enabling the isolation of the $S_{i j}(Q)$ partial structure factors and allowing the determination of the partial pair correlation functions $g_{i j}(r)$.

\subsection{Chemical composition of the studied glasses}

Before entering into the details of oxide glass structure, we will describe the chemical composition of the main materials with which we are concerned. Then, the explanations about the glass network arrangement shall be read in the light of the chemical elements present in the studied mirror substrates. Currently, three different borosilicate glasses are used for neutron guide manufacturing, named Borofloat, N-ZK7, and N-BK7. All of them are borosilicates with a similar content of boron that provides the needed neutron absorption ability. The main difference amongst them comes from their manufacturing process. Borofloat plates are melted on a tin bath that provides a naturally suitable thickness and very good surface roughness (float method [84]) for neutron mirror manufacturing. Before coating a neutron mirror on a Borofloat plate, one only needs to polish it once with a cerium oxide suspension. On the other hand, N-ZK7 and N-BK7 are optical grade borosilicate glasses. They are melted in the shape of large cubes and cooled down slowly to relax any internal stresses in order to avoid light birefringence. For these materials, guide manufacturers have to cut plates out of the raw products and operate several polishing steps to reach the needed surface roughness and waviness. In addition to those three materials, S-BLS7 is also studied in this thesis. It is a candidate for future guide manufacturing and this thesis work has been conceived to investigate its behaviour under irradiation.

Knowing the chemical composition of our glasses will allow us to understand their structure and possibly relate it to their behaviour under neutron flux. In addition, knowing this information is mandatory before any irradiation experiments occur. At ILL, before putting a sample under thermal neutron flux, one has to justify that the radionuclide produced will make it too active to be manipulated, especially in the case of the high fluence experiments that will be described later in the document.

The chemical analyses were carried out by the Institut des Sciences Analytiques in Villeurbanne, France. The aim was to get information on the chemical composition of each kind of glass, as well as details pertaining to impurities which may be relevant for radiation protection reasons. Table 2.1 displays the main elements that interest us in term of glass network properties.

In addition to the chemical analysis, Table 2.1 lists the density measured by the Archimedes method, macroscopic absorption cross section, and the corresponding neutron mean free path for each material. Taking into account the large ${ }^{10} \mathrm{~B}(\mathrm{n}, \alpha)$ cross section, we have only considered this variable to calculate the global absorption cross section. 


\begin{tabular}{c|cccc}
\hline mol. \% & Borofloat & N-ZK7 & N-BK7 & S-BSL7 \\
\hline $\mathrm{SiO}_{2}$ & 82 & 66 & 73 & 73.5 \\
$\mathrm{~B}_{2} \mathrm{O}_{3}$ & 12 & 11.5 & 10 & 9.5 \\
\hline $\mathrm{Na}_{2} \mathrm{O}$ & 4 & 7.5 & 10 & 10 \\
$\mathrm{~K}_{2} \mathrm{O}$ & 0.5 & - & 5 & 5.5 \\
$\mathrm{CaO}$ & - & 1 & 0.25 & - \\
$\mathrm{BaO}$ & - & - & 0.25 & 0.5 \\
\hline $\mathrm{MgO}$ & - & - & 1.25 & - \\
$\mathrm{Al}_{2} \mathrm{O}_{3}$ & 1.5 & 4 & 0.25 & - \\
$\mathrm{ZnO}$ & - & 10 & - & 1 \\
\hline \hline Density g/cm & 2.205 & 2.469 & 2.4914 & 2.504 \\
$\mathrm{~T}_{\mathrm{g}}\left({ }^{\circ} \mathrm{C}\right)$ & 525 & 539 & 557 & 576 \\
$\Sigma_{a}\left(\mathrm{~cm}^{-1}\right)$ & 3.97 & 4.16 & 3.71 & 3.46 \\
$\lambda\left(\mathrm{cm}^{3}\right.$ & 0.25 & 0.24 & 0.27 & 0.29 \\
\hline
\end{tabular}

Table 2.1: Chemical composition of glass, values in molecular percent, Institut des Sciences Analytiques measurements (Villeurbanne, France). At the bottom of the table additional properties of the measured materials can be found

Also, from this table one can gather an idea of the thermal neutron shielding capacity of every glass. The absorption law of a collimated beam passing through material is as follows,

$$
\frac{d I}{d x}=I_{0} \cdot e^{-\frac{x}{\lambda}}
$$

where $I_{0}$ is the initial beam intensity, $x$ is the distance travelled in the material, and $\lambda$ is the mean free path. The application of that formula shows that after $x=3 \lambda$, the beam intensity has been divided by a factor of 20 . Then, in the case of a guide frontal irradiation, a neutron will roughly penetrate less than a centimetre. In turn, we now consider the grazing incidence condition for a " $m=2$ " thermal guide and assume a neutron beam with wavelength $1.8 \AA$ having a divergence equal to three fourths of the critical angle, that is $0.27^{\circ}$. The beam intensity as a function of the depth $u$ from the surface is now,

$$
\frac{d I}{d u}=I_{0} \cdot e^{-\frac{u}{\sin \left(0.27^{\circ}\right) \cdot \lambda}}
$$

from where we infer that the greatest glass depth the beam can reach is around $0.05 \mathrm{~mm}$. This value will be calculated with more accuracy in another section of the document. This first glance at which part of the mirror substrate is affected by the moderated neutrons highlights that only a small amount of the total thickness is irradiated. Indeed, the typical glass plate thickness ranges from 9 to $15 \mathrm{~mm}$. 


\subsection{Internal structure of an alkali-borosilicate glass}

\subsubsection{The role of the different oxides}

The nine oxides listed in Table 2.1 are grouped into three classes, which account for the role played by the different kinds oxide compounds on the glass network structure. In short, such groups comprise the

- Network formers, which can generate a glassy network by themselves. In our case, these are $\mathrm{SiO}_{2}$ and $\mathrm{B}_{2} \mathrm{O}_{3}$;

- Network modifiers which, once incorporated into the network, induce substantial modifications of its structure. These are mostly alkali and alkali-earth oxides such as $\mathrm{Na}_{2} \mathrm{O}, \mathrm{K}_{2} \mathrm{O}, \mathrm{CaO}$ and $\mathrm{BaO}$;

- Intermediate ions, which have properties belonging to both of the above cited groups. For the glasses we are concerned with, the most important are $\mathrm{Al}_{2} \mathrm{O}_{3}$ and $\mathrm{ZnO}$.

Let us now go into more detail concerning this topic:

\section{Network formers}

Only few oxide molecules can form a glass when passing from the liquid state to the solid state. As explained earlier, this ability is highly dependent on the liquid viscosity, that is, a liquid vitrifies, if its components cannot arrange themselves into a crystalline structure. The most widely studied glass network former is $\mathrm{SiO}_{2}$, which, once crystallized, gives rise to a variety of crystal forms (quartz, cristobalite, trydimite, etc.) depending upon the thermodynamic conditions. As silicon cations are $4^{+}$charged and oxygen anions $2^{-}$, they form elementary tetrahedrons with one silicon ion in its center and four oxygen ions in the corners [16], see Fig. 2.1a. The oxygens are linked to two silicons to ensure the electro-neutrality. The 3D network resulting from the assembly of tetrahedrons is fairly rigid as can be inferred from the high glass transition and melting temperatures, see Table 2.2.

The other network former that interests us is $\mathrm{B}_{2} \mathrm{O}_{3}$, which assembles as triangles with a boron cation $\left(3^{+}\right)$in the center and oxygen anions on the corners, see Fig. 2.1b. Because of its $2 \mathrm{D}$ elementary arrangement, $\mathrm{B}_{2} \mathrm{O}_{3}$ network tends to be much more flexible that the silicate. For that reason, $\mathrm{T}_{\mathrm{G}}$ of pure borate glass is much lower than the one of pure silicate. The assembly of several $\mathrm{BO}_{3}$ triangles form groups with a well-defined shape named Boroxol rings [114], see Fig. 2.3a.

Finally, for both kinds of network formers, the silicon tetrahedra and the boroxol groups assemble into ring-shaped structures, the size of which varies from one to the other. This variability explains the absence for long range order. Even though the first neighbours of $\mathrm{Si}$ or B are well defined, one cannot guess the exact position of further atoms, an issue that usually is tackled by recourse to radiation scattering techniques. 


\begin{tabular}{ccc}
\hline & $\mathrm{T}_{\mathrm{L}}(\mathrm{K})$ & $\mathrm{T}_{\mathrm{G}}(\mathrm{K})$ \\
\hline $\mathrm{SiO}_{2}$ & 1996 & 1473 \\
$\mathrm{~B}_{2} \mathrm{O}_{3}$ & 1386 & 900 \\
\hline
\end{tabular}

Table 2.2: Melting and glass transition temperature of two network formers

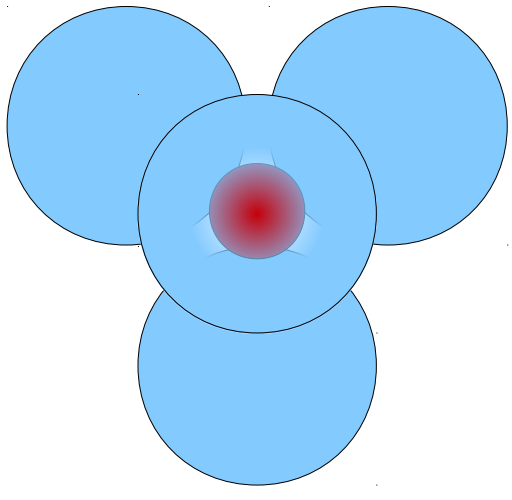

(a) $\mathrm{SiO}_{4}$ tetrahedron

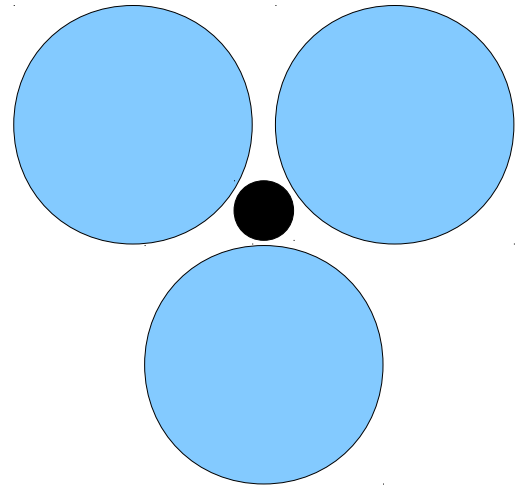

(b) $\mathrm{BO}_{3}$ triangle

Figure 2.1: 2D views of elementary silicon and boron oxide structure in glasses, view from above. Oxygen anions in blue, silicon cation in red, and boron cations in black.

\section{Network modifiers}

It is thanks to these chemical elements that glasses can be produced at a relatively low temperature compared with the glass-transition range of pure silica-glass. Network modifiers classify most of them with the alkalis and alkaline-earths $\left(\mathrm{Li}_{2} \mathrm{O}, \mathrm{Na}_{2} \mathrm{O}, \mathrm{K}_{2} \mathrm{O}\right.$, $\mathrm{CaO}, \mathrm{BaO}$, etc). In the case of silica glass, one network modifier molecule will break two Si-O bonds and create two non-bridging oxygens(NBO) linked on one side to a silicon atom and on the other to the modifier. To compensate two NBOs, it will need two sodium ions but only one calcium ion. The proportion of silicon ions linked with $n$ bridging oxygens was often named $Q_{n}$. As a result the modified network becomes gets more flexible and its melting temperature is significantly lowered [16]. The effects of progressively adding alkalis to pure silica has been quantified by Maekawa et al. [66], and show that Q4 is gradually replaced by Q3, Q2, and finally Q1, see Fig. 2.2. Two mechanisms are in operation when adding network modifiers to borate glass: first, they create $\mathrm{BO}_{4}$ tetrahedra and then generate NBOs [25, 119].

In addition, network modifiers will change the glass density and may deform its network. If one compares the effects of $\mathrm{Na}_{2} \mathrm{O}$ and $\mathrm{K}_{2} \mathrm{O}$ on silica, one can see that sodium has nearly no effect on the molar volume, while potassium induces a swelling of the network 96 . However, they increase in a similar way to the material density: $2.45 \mathrm{~g} / \mathrm{cm}^{3}$ for $25 \mathrm{~mol} . \%$ of either $\mathrm{Na}_{2} \mathrm{O}$ or $\mathrm{K}_{2} \mathrm{O}$ [101].

\section{Intermediate ions}

These elements in terms of glass network production are located somewhat between network formers and modifiers. In addition to $\mathrm{Al}_{2} \mathrm{O}_{3}$ and $\mathrm{ZnO}$ mentioned previously, $\mathrm{BeO}$ 


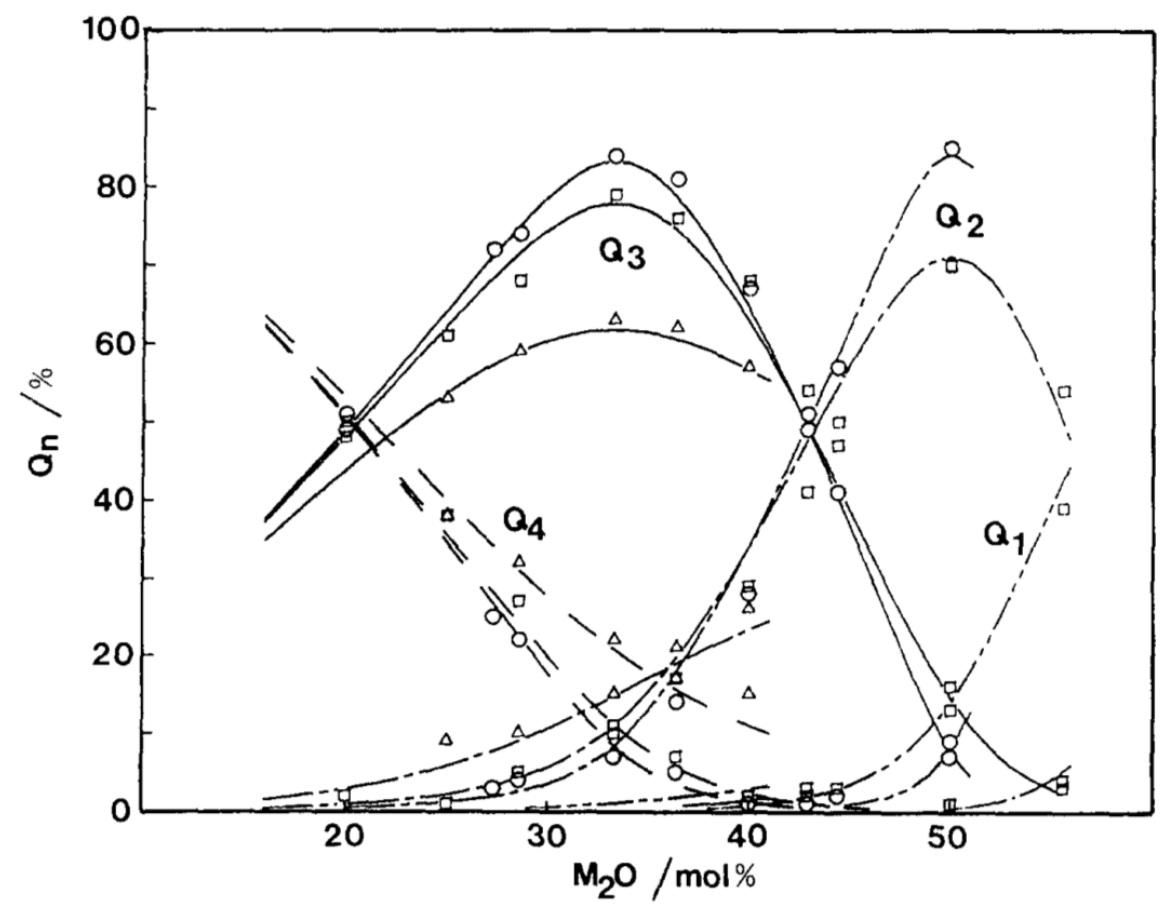

Figure 2.2: Experimentally determined $Q_{n}$ distribution in lithium $(\triangle)$, sodium (o) and potassium ( $\square$ ) silicate glasses as a function of mol\% of alkali oxide. From [66].

and $\mathrm{MgO}$ are also part of this family. These elements are not able to vitrify by themselves, but may either contribute to the network formation or modify it. The effect depends on the concentration of alkali in the glass.

Concerning zinc oxide, the study from Hurt and Phillips [53] on $\mathrm{Na}_{2} \mathrm{O}-\mathrm{ZnO}-\mathrm{SiO}_{2}$ glass systems showed that, at low zinc content, there are mainly fourfold coordinated atoms which incorporate into the silica network. By contrast, at higher concentrations, zinc turns into an octahedral coordination, which acts as a network modifier. Further studies carried out by Rosenthal and Garofalini [92] lend further support to such a view, although their study inferred that tetrahedrally coordinated zinc atoms were also modifying the amorphous silica structure, a conclusion which may possibly arise from the larger size of $\mathrm{Zn}^{2+}$ ion compared to $\mathrm{Si}^{4+}: 0.88 \AA$ versus $0.4 \AA$, respectively.

The behaviour of $\mathrm{Al}_{2} \mathrm{O}_{3}$ is similar to what has been just described. When added to pure silica glass, it adopts a 6 -fold coordination and acts as a network modifier. However, in alkali silica glass, it turns into tetrahedral coordination and can replace Si that have alkali in their surrounding. At constant alkali concentration, each $\mathrm{Al}_{2} \mathrm{O}_{3}$ added will turn two NBOs into bridging oxygens [26].

\subsubsection{Borosilicate based glasses}

As explained above, $\mathrm{SiO}_{2}$ and $\mathrm{B}_{2} \mathrm{O}_{3}$ are two glass network formers. Mixing them together also produces glass, but with specific properties. $\mathrm{B}_{2} \mathrm{O}_{3}$ lowers the glass transition point by reducing the viscosity of the melt. The reason is the fundamental difference in the elementary network unit: while $\mathrm{SiO}_{2}$ forms stiff tetrahedra, the $\mathrm{BO}_{3}$ triangles bring flexibility to the silica network. The present subsection will be focused on their network 
structure and how it is shaped by network modifiers and intermediate ions. In order to ease the reflections on glass chemistry, parameters have been defined: $R=\frac{\left[\mathrm{Na}_{2} \mathrm{O}\right]}{\left[\mathrm{B}_{2} \mathrm{O}_{3}\right]}$ and $K=\frac{\left[\mathrm{SiO}_{2}\right]}{\left[\mathrm{B}_{2} \mathrm{O}_{3}\right]}$, where the " $[\mathrm{x}]$ " represents the molar concentrations of the oxide specie $\mathrm{x}$.

\section{Phase separation}

According to [48], the $\mathrm{SiO}_{2}-\mathrm{B}_{2} \mathrm{O}_{3}$ systems are compatible and should not exhibit any phase separation. Later, Martens and Müller-Warmuth [70 agreed on this and their NMR study showed that the ${ }^{11} \mathrm{~B}$ peak position was influenced by the boron content, which was interpreted as a fact proving that boron was affected by a silicon environment. In a system with $\mathrm{SiO}_{2}$ and $\mathrm{B}_{2} \mathrm{O}_{3}$ well apart, the addition of boron in the glass would not have affected the NMR signal of ${ }^{11} \mathrm{~B}$. This being said, the presence of a third or more elements leads to the emergence of an immiscibility region and generates two phases by demixing the two networks. This means that the usual alkali-borosilicate glasses may be considered as a two phase system; one having an alkali-borate rich region and another silicon rich region [70,86, 91]. The concept of separated phases is important in the frame of this study for two reasons, which concern

- The existence of unmixed phases, which is the equivalent of metal microstructure. As vitreous materials have no long range order, only three things can be characterized: its atomic arrangement of the network, the detailed shapes of silicon and boron structural groups, and the existence of phases with different chemical composition.

- The radiation damages resulting from recoiling nuclei that have previously impinged on the glass structure. In this case, a ballistic mixing of the material will occur, the consequences of which will be highly dependent on material homogeneity or heterogeneity.

An industrial application of the phenomenon described above is the Vycor glass, developed by H.P. Hood and M.E. Nordberg, aiming to produce an almost pure silicon oxide glass. The technique is based on the production at a relatively low temperature of a sodium borosilicate having two intermixed phases, one of them containing $95 \% \mathrm{SiO}_{2}$. Immersion in sulphuric acid removes the borate phase, leaving the pure silicate that can be compacted by a heat treatment around $1000{ }^{\circ} \mathrm{C}$. On the same topic, Elmer et al. [41] studied the effect of heat treatment on the demixing in sodium borosilicate. They showed that the size of the silica-rich phase was increasing with the increase of time and temperature of the heat treatment.

\section{Effect of alkalis addition}

Adding network modifiers to a borosilicate melt should logically modify silicate and borate elements as a function of the stoichiometry. Such modification concerns the generation of NBOs on silicon tetrahedra concomitant with turning $\mathrm{BO}_{3}$ triangles into $\mathrm{BO}_{4}$ tetrahedra. Several NMR studies have, however, shown that this is not the case when considering ternary systems. Yun and Bray [119], and later Dell et al. [33], have shown that sodium preferentially transforms $\mathrm{BO}_{3}$ into $\mathrm{BO}_{4}$ rather than creating NBOs for silicon. They have shown that for $K \leq 8$ and $R \leq 0.5$, each sodium ion added creates $\mathrm{BO}_{4}$ that assembles into diborate structures, see Fig. 2.3b. Above $R=0.5$, the added sodium would start to 


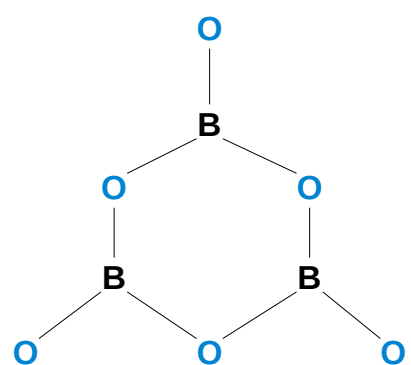

(a) $2 \mathrm{D}$ view of a boroxol group 114

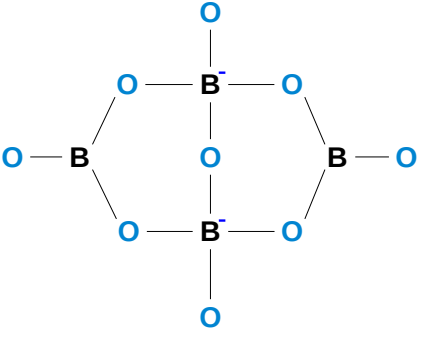

(b) 2D view of a diborate group [114, sodium charge compensating atoms are not shown

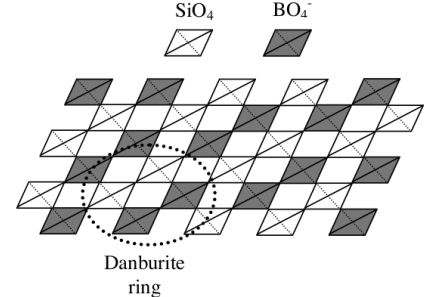

(c) Crystalline Danburite structure 69

break those structures and boron would be incorporated into $\mathrm{BSi}_{4} \mathrm{O}_{10}$ units. However, Martens and Müller-Warmuth [70] moderated this model by showing that alkali cations were not solely absorbed by the borate network. The significance of this result for the complex glasses we are interested in warns us to take the Yun and Bray model with care when analyzing data. It certainly can give an estimate of the $\mathrm{BO}_{3} / \mathrm{BO}_{4}$ ratio, but should only be taken an estimate.

In a more recent study using Raman sectroscopy, Manara et al. [69] compared the sodium borosilicate spectrum with Raman signals coming from crystalline danburite. This mineral havs the composition $\mathrm{CaO}-\mathrm{B}_{2} \mathrm{O}_{3}-2 \mathrm{SiO}_{2}$, where one calcium ion compensates for the charge of two $\mathrm{BO}_{4}$, see Fig. 2.3c. They showed in their article that a broad Raman peak produced by the vitreous material could correspond to danburite ring structures. In the case of an NBS glass, each calcium ion has to be replaced by two sodium ions. The advantage of this type of measurement is that it allows researchers to clearly detect the existence of a borosilicate network.

\section{The aluminium preference}

Because of its intermediate oxide properties, $\mathrm{Al}_{2} \mathrm{O}_{3}$ plays a complex role when mixed within an alkali borosilicate matrix. The effects of this component are worth mentioning, as it is a major constituent of Borofloat glass, see Table 2.1 on p.24. As explained before, aluminium ions in low quantity take fourfold coordination and reduce NBO content. El-Damrawi et al. [39] have shown that for a $\mathrm{Na}_{2} \mathrm{O}-\mathrm{Al}_{2} \mathrm{O}_{3}-\mathrm{B}_{2} \mathrm{O}_{3}-\mathrm{SiO}_{2}$ system, network modifiers are preferentially incorporated to generate $\mathrm{AlO}_{4}$ tetrahedra, rather than converting $\mathrm{BO}_{3}$ to $\mathrm{BO}_{4}$. In a similar way, the number of NBOs per silicon atom is strongly reduced by the $\mathrm{Al}_{2} \mathrm{O}_{3}$. Du and Stebbins [36] studied in depth the effects of $\mathrm{Al}_{2} \mathrm{O}_{3}$ on boron speciation. They suggested that, because of its high field strength, aluminium cations favours the formation of a $\mathrm{NBO}$ around $\mathrm{BO}_{3}$ rather than the transformation in $\mathrm{BO}_{4}$. 


\subsection{Sources of radiation damage: gamma rays, fast and moderated neutrons}

When a guide is used under normal operating conditions, there are three primary radiation ageing sources that deteriorate the glass substrate:

- Gamma rays. Some of them come from the neutron source, others from absorption reactions on neighbouring elements: reactor vessel, beam tube, supermirror etc.

- Fast neutrons coming from the primary source. For the sake of beam intensity, the moderator size is not infinite and a small part of the fission, or spallation, spectrum might not be moderated.

- Moderated neutrons, either thermal or cold, that are transported through the guide and bounce several times across the mirror.

In addition, there are secondary ageing sources that come from the capture reaction in the glass substrate. The moderated neutrons that penetrate the material might be absorbed and produce ionizing particles through reactions such as $(n, \gamma),(n, p),(n, \alpha)$, etc. This is particularly relevant in the case of borosilicate glass, where the presence of boron is purposeful to enable the guide to capture runaway neutrons. Considering the composition of the borosilicates we are interested in, one reaction is prevailing over the others because of its high cross section: ${ }^{10} \mathrm{~B}(\mathrm{n}, \alpha){ }^{7} \mathrm{Li}:{ }^{10} \mathrm{~B}+\mathrm{n} \rightarrow{ }^{7} \mathrm{Li}+\alpha$. In fact, the cross-section for neutron capture on boron-10 (20\% natural abundance in boron) is comparatively huge (3980 barn) for neutrons with thermal energies $(0.025 \mathrm{eV})$. For cold neutrons, the cross-section is even higher: $\left.\sigma \propto 1 / \sqrt{(} E_{n}\right)$. In $6 \%$ of the neutron captures, the decay proceeds to the ground state of ${ }^{7} \mathrm{Li}$ while $94 \%$ of decays proceed via the excited state of ${ }^{7} \mathrm{Li}$ and are accompanied by emissions of a $478 \mathrm{keV}$ gamma ray. For both cases, the $Q$ value (i.e the minimum possible energy released) of the reaction is large compared to the incoming neutron energy, and the total kinetic energy of the resulting products $\alpha$ and ${ }^{7} \mathrm{Li}$ basically equal the reaction energy. Conservation of energy and momentum gives values of $1.16 \mathrm{MeV}$ for ${ }^{7} \mathrm{Li}$ and $1.78 \mathrm{MeV}$ for the $\alpha$ particle for the ground state reaction and $0.84 \mathrm{MeV}$ for ${ }^{7} \mathrm{Li}$ and $1.47 \mathrm{MeV}$ for the $\alpha$ particle generated by the gamma-ray accompanied reaction. Both charged particles have high linear energy transfer and short range, hence, they deposit their kinetic energy locally within few ten micrometers from the point of capture.

Finally, another ionizing source to consider results from nuclear absorption on the other nuclei of the glass. The resulting decay reactions are, in most cases, $\beta$ disintegration. However, if one considers the different absorption cross-sections in the material, it can be found that for Borofloat, more than $99.95 \%$ of the absorption reactions occur on ${ }^{10} \mathrm{~B}$ nuclei. Therefore, for all practical purposes, we consider that the thermal and cold neutron absorptions only happen on ${ }^{10} \mathrm{~B}$ nuclei that mostly take place on the first $0.05 \mathrm{~mm}$, see equation $2.13 \mathrm{p} 24$.

When analysing the guide incidents registered at the different neutron research centres, it is apparent that the mechanical source of damage is the generation of stress in the glass plates. Such stress can only result from a heterogeneous shrinking or swelling of the material. In the case of the H25 or H113 failures, the figures (p. 16 and 18) show the first 
millimetre of glass peeling off from the bulk. Logically, the affected layer should be of the same order of magnitude as the detached layer. If we consider fast neutrons and $\gamma$ rays, they will deposit energy on a longer scale. In fact, for Borofloat, the mean free path for fast neutrons is around $4 \mathrm{~cm}$ and the linear absorption coefficient is around $0.15 \mathrm{~cm}^{-1}$. The nuclear reaction responsible of the glass modification has hence been ascribed to the $(\mathrm{n}, \alpha)$ reactions on ${ }^{10} \mathrm{~B}$ nuclei.

\subsection{Glass behaviour under irradiation}

The following section will deal with the effect of different kinds of radiation on the alkali borosilicate structure described earlier. Even though ${ }^{10} \mathrm{~B}(\mathrm{n}, \alpha)$ reactions seem to be the most deleterious for the glass structure, it is worth mentioning the effects of $\gamma$ rays and fast neutrons. Indeed, the damage induced by the $\alpha$ particle and the lithium ion can be seen as a mixture of ionization track and ballistic mixing, corresponding to $\gamma$ and fast neutron damages, respectively.

\subsubsection{Gamma rays}

This type of radiation is present all along the guides. $\gamma$ rays in the first meters of each beam line mostly come from the neutron source, while further downstream it comes from the neutron capture reactions in the super-mirrors and the glass itself. In both cases, the whole thickness of the mirror plates feels an homogeneous $\gamma$ flux. Such rays can interact with the electron clouds in the material through the Compton interaction [7]. Scattered electrons thus become a further source of radiation damage. The most visible results of such an interaction is, in some ways, similar to the well studied cases of formation of colour centres in crystals as mentioned in Ref. [77]. Apart from this, one has to take into account the radiolitic power of such electrons, which by means of radio-chemical reactions may lead to modifications in glass structure. The net result of such latter interactions would be a short range rearrangement of the bonds, leading to atomic displacements.

In terms of stress generation, which is the topic that interests us, Shelby [100] studied the macroscopic effect of ${ }^{60} \mathrm{Co} \gamma$ rays on different borosilicates with varying compositions around $75 \%$ for $\mathrm{SiO}_{2}, 12 \%$ for $\mathrm{B}_{2} \mathrm{O}_{3}, 4 \%$ for alkali, and $3 \%$ for $\mathrm{Al}_{2} \mathrm{O}_{3}$. The results show that, as can be seen on Fig. 2.4 the density increases with the received dose. A look at the figure shows that the slopes are pretty similar for all the examined compositions.

On the grounds of the results commented on above, we do expect that $\gamma$ radiation will modify the borosilicate glasses employed in this study. However, for what really matters to us, recall that the small attenuation power of these materials for this kind of radiation will cause the density to vary homogeneously across the glass plate and thus should not induce any considerable stress in the material.

\subsubsection{Fast neutrons}

Because of electro neutrality, fast neutrons will solely damage the material through ballistic interactions. Neutron sources aiming at producing moderated neutrons are designed to avoid the transport of fast neutrons outside of the moderator as much as possible. For that reason, at the guide entrance, the fast flux is generally several orders of magnitude 


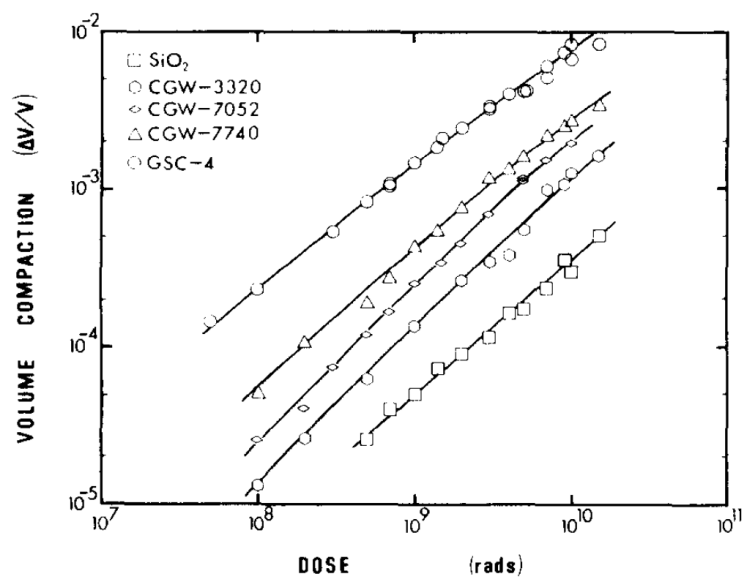

Figure 2.4: Alkali borosilicate compaction under ${ }^{60} \mathrm{Co} \gamma$ irradiation. From [100].

below the thermal one. However, it is worth mentioning their effects since such energetic particles contribute to modifying the materials on the first meters of any guide.

Primak [88] worked on the macroscopic effects of fast neutrons on pure vitreous silica and showed that these particles tend to compact the silica structure between 2 and $3 \%$ for a received flux around $2 \times 10^{20} \mathrm{n} / \mathrm{cm}^{2} / \mathrm{s}$. Later studies by Wright et al. [116] investigated the deep structural evolution of vitreous silica. They showed that the silicon rings, typical for glass, were getting smaller with increasing dose. In addition, $\mathrm{Si}-\mathrm{O}$ bond length increased and $\mathrm{Si}-\mathrm{O}-\mathrm{Si}$ was reduced by $9.5^{\circ}$.

Such behaviour under fast neutron flux can surely apply to borosilicate glasses with high silicon content, as in our case. However, considering the typical neutron energy spectrum at a guide entrance $\left(2.5 \%\right.$ epithermal and $0.2 \%$ fast ${ }^{1}$ for $\left.\mathrm{H} 5\right)$, researchers would need a thousand years of operation to see its properties really modified by a mix of fast and epithermal neutrons. This kind of irradiation is then neglected to a good approximation.

\subsubsection{Thermal neutrons}

For most materials, the damages induced by thermal neutrons are a slow process. Through radiative capture and radio-active decay they turn aluminium into silicon. It is in fact the most problematic issue for the ILL's beam tube lifetime limit. In a float glass, considering the different absorption cross sections, most of the reactions will involve oxygen, sodium, potassium, aluminium, etc. If one considers the maximum flux achieved in a guide, around $2 \times 10^{11} \mathrm{n} / \mathrm{cm}^{2} / \mathrm{s}$, with an absorption cross section $\sigma=5$ barn and $5 \times 10^{21}$ at $/ \mathrm{cm}^{3}$ for typical sodium content in a glass, it will need one full year to transmute $30 \mathrm{ppm}$ of sodium into magnesium. From that calculation, one can see that the chemical modification of glass guides under thermal neutron flux is not fast enough to be considered as a source of ageing. For that reason, the important capture reaction left for this study is ${ }^{10} \mathrm{~B}(\mathrm{n}, \alpha) \mathrm{Li}$, already commented on above.

\footnotetext{
${ }^{1}$ Usually it is considered that fast neutrons have an energy above $1 \mathrm{MeV}$ while epithermal ones are between $1 \mathrm{eV}$ and $1 \mathrm{MeV}$.
} 


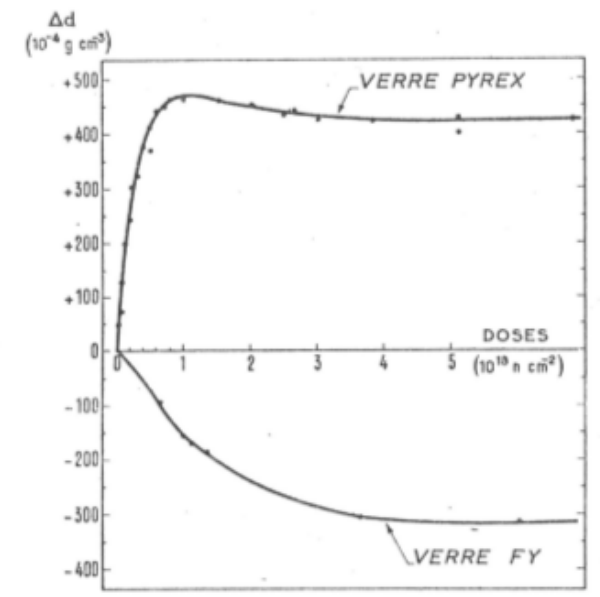

(a) Density variation of lead glass ("VERRE FY") and Pyrex as a function of thermal neutron fluence, From [78].

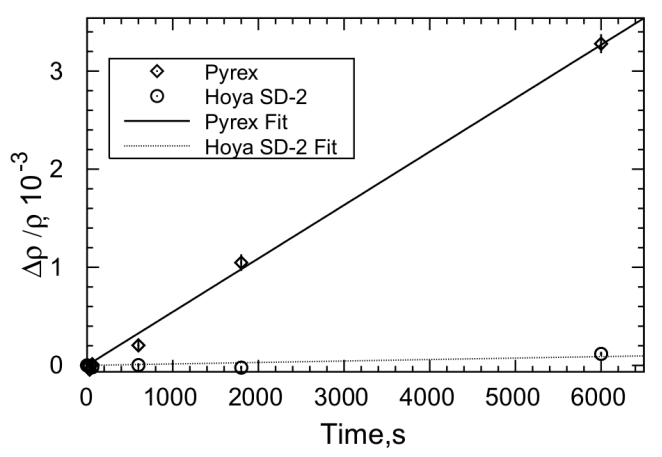

(b) Density variation of Pyrex and Hoya SD-2 as a function of thermal neutron fluence. Thermal flux $=6.7 \times 10^{12} \mathrm{n} / \mathrm{cm}^{2} / \mathrm{s}$, From 8 .

\section{Glass contraction}

Mylonas and Truell [76] found that a large glass plate densified due to the stresses induced by an heterogeneous neutron flux hitting the sample. From their experiment, they extracted an average free volume contraction per $(\mathrm{n}, \alpha)$ reaction,

$$
\left\langle\Delta V^{\prime}\right\rangle \cong-3.6 \cdot 10^{-19} \frac{\mathrm{cm}^{3}}{{ }^{10} B(n, \alpha)}
$$

The glass they had studied was a borosilicate with $32.8 \%, 8.24 \%$ and $1.1 \%$ of silicon, boron and alkali respectively. Even though this composition is similar to our glass, one should mention that the alkali content was much lower than in our case, which has an important effect on the glass structure as it has been explained above. Later, Paymal [78 published a study on the effects of $(n, \alpha)$ reactions on borosilicate glasses, especially Pyrex. This material and Borofloat have a similar composition and follow close manufacturing processes. The results from the density measurements on two glasses are shown in Fig. 2.5a. In the study, they related the shrinking of Pyrex to the fact that it was rich in silica $\left(82 \mathrm{~mol} . \% \mathrm{SiO}_{2}\right)$ and that vitreous silica shrinks under radiation. For the other glass studied containing 10.4 mol.\% of PbO (a network modifier), the silica content is reduced and does not show such striking behaviour under flux. Allred et al. [8] have shown similar behaviour for 2000s Pyrex. They also tested another glass with a lower content of boron and higher content of $\mathrm{Al}_{2} \mathrm{O}_{3}$, which showed far less shrinking under thermal flux.

\section{Phase segregation}

As mentioned in the above sections, the evolution under neutron flux of the heterogeneities of alkali-borosilicate glasses is expected to play an important role as far as the mechanical properties of the material are concerned. Prado et al. [86, 87] studied how this type of material was unmixing and how the phases were evolving under thermal neutron flux. 


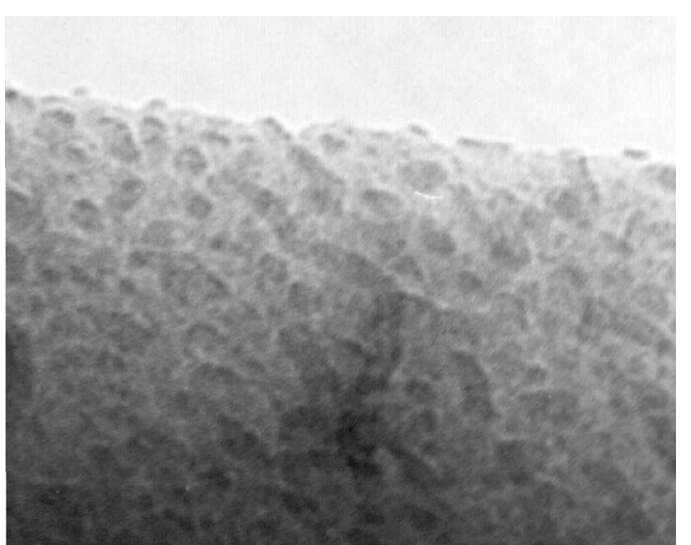

(a) Phase segregation in aluminoborosilciate seen by TEM imaging, From 87. Photograph width: 150nm.

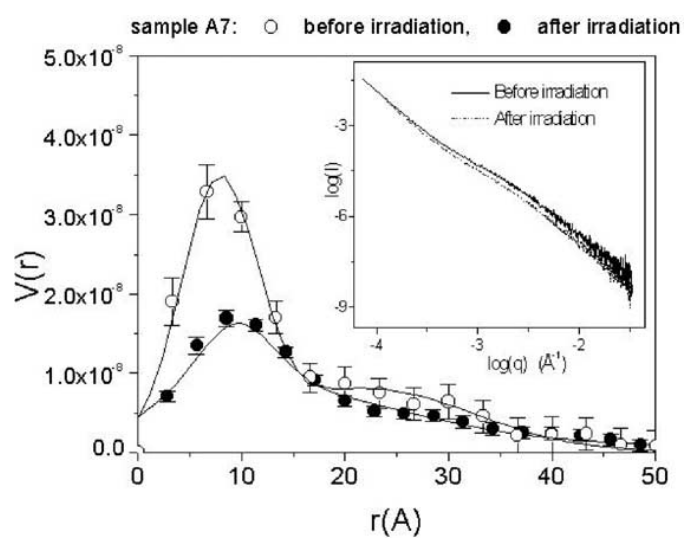

(b) Particle distribution in aluminoborosilciate glass before and after irradiation. Calculcated from SAXS intensities, From 87.

Figure 2.6: Behaviour of phase separation in alkali-borosilicate glass under irradiation

The material studied exhibited a silicon rich phase and an alkali-borate rich phase, which seems to be the case for materials with an equivalent composition. They linked the global density change of the material to the ballistic mixing induced by the (n, $\alpha$ ) reactions. Their idea was that it could induce a transport of sodium in the silicate phase that would modify its chemical composition and a compaction of its structure. This phenomenon was being countered by the creation of voids and interstitials that tend to provoke swelling of the glass structure. Figures 2.6a and 2.6b show the phases in the glass before irradiation and the evolution of their size under radiation respectively.

\section{Helium cavities formation}

As helium is a direct product of the $(n, \alpha)$ reactions, it is worth considering the possibility of cavity, or "bubble", formation caused by electron capture from the $\alpha$ particles. However, Inagaki et al. [54 and later Peuget et al. [82 did not detect significant helium bubbles at room temperature under a dose of $10^{19} \alpha / \mathrm{g}$. The reaction rate limit at which glass plates break shall be compared to this value in order to have a first estimate of the possibility of helium bubble generation. However, several studies [44,68, 73 have shown that helium bubbles do start to appear if the temperature is raised to values above $T_{\mathrm{g}}$. Under such conditions, the low viscosity allows the deformation of glass networks that accommodate helium atom clusters.

\section{Structural behaviour}

The literature presents numerous studies on the structural evolution of borosilicate (BS) glass under radiation, with ionizing particles ranging from helium to gold ions. Under certain conditions, the effects of $(n, \alpha)$ reactions can be related to damages induced by heavy ions or pure helium bombardment. The $\alpha$ and lithium particles produced by the ${ }^{10} \mathrm{~B}$ neutron capture have, on a first approximation, a similar weight and kinetic energy. Consequently, helium ion sputtering studies can be considered relevant to our topic. 
Yang et al. [118] compared the effects of helium ions and electrons on a BS glass. They showed that electrons were increasing the network polymerization by reducing $\mathrm{Q}_{2}$ and increasing $\mathrm{Q}_{3}$ and $\mathrm{Q}_{4}$, whereas He ions were not obviously changing these parameters. They explained the results as if the ballistic mixing created by the He stopping counter balanced the effects of electronic slowing down. On the contrary, He ions were much more efficient than the electrons in modifying the material hardness.

Peuget et al. [83] studied the structural effects of radiation on a ternary NBS glass by means of neutron and ion beams and compared this to the effects brought forward by different quenching rates during the glass formation. They showed that ion irradiation (thermal spikes) had similar consequences on the glass network than fast quenching (above $3 \times 10^{4} \mathrm{~K} / \mathrm{min}$ ). The results were fully coherent with the early description of thermal spikes given by Paymal and Bonnaud [78]. The results, as far as the boron coordination characteristics are concerned, show that xenon ion irradiation and fast quenching were both generating $\mathrm{BO}_{3}$ units from $\mathrm{BO}_{4}$, see Fig. 2.7b. Raman and ${ }^{29} \mathrm{Si}$ MAS-NMR spectra have shown that $\mathrm{Xe}, \mathrm{Kr}$, and neutron irradiation were increasing the $\mathrm{Q}_{3}$ concentration, see Fig. $2.7 \mathrm{c}$.

\subsection{Mirror substrate splintering fluence}

The study described here has been carried out at the ILL following the H113 and H17 neutron guide incidents. The institute's technical service wanted to have an idea of the neutron flux that could be sustained by different substrates when irradiated heterogeneously. The fluence found where the substrate is cracking could be chosen as a limit above which a neutron guide should not be used further.

\subsubsection{Experimental procedure}

For such a study, neutron fluxes provided by the H112 and H21 guides have been used [30]. They provided a collimated flux of $6 \times 10^{10}$ cold neutrons $/ \mathrm{cm}^{2} / \mathrm{s}$ and $1.1 \times 10^{9}$ thermal neutrons $/ \mathrm{cm}^{2} / \mathrm{s}$, respectively. These flux values have been measured by means of gold foil activation techniques [104], hence we quote the equivalent thermal neutron capture flux here for cold neutrons.

The experimental setup was built to mimic the configuration a glass faces when installed within a guide, namely neutron incidence under a small angle. The result is that neutrons are stopped in a small layer right under the mirror and induce an heterogeneous dose in the glass substrate. However, for the sake of reaching a high dose more quickly, a $4.3^{\circ}$ average incidence angle was set, see Fig 2.8. The net result of such an arrangement is that all neutrons within the beam will cross through the mirror and penetrate in the glass matrix. In comparison, the neutrons which are not reflected by the mirror have a maximum angle of about $3^{\circ}$ (a typical value for a $m=2$ cold neutron guide and neutrons with wavelength of $15 \AA$ ).

An apparatus able to accommodate up to 30 samples was built for the purpose. The frontal irradiation values quoted above yield values for the whole sample surface of $4.5 \times 10^{9} \mathrm{n} / \mathrm{cm}^{2} / \mathrm{s}$ and $8.2 \times 10^{7} \mathrm{n} / \mathrm{cm} 2 / \mathrm{s}$ for $\mathrm{H} 112$ and $\mathrm{H} 21$ respectively. Table 2.3 summarizes the information regarding the irradiation setups. 


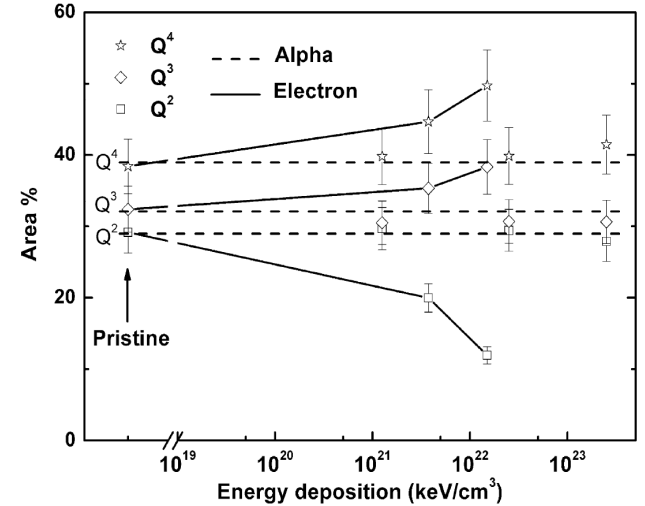

(a) Variations of relative area of the $\mathrm{Q}_{n}$ bands versus energy deposition. Areas determined by fitting Raman spectra, with one gaussian per $\mathrm{Q}_{\mathrm{n}}$. From [118].

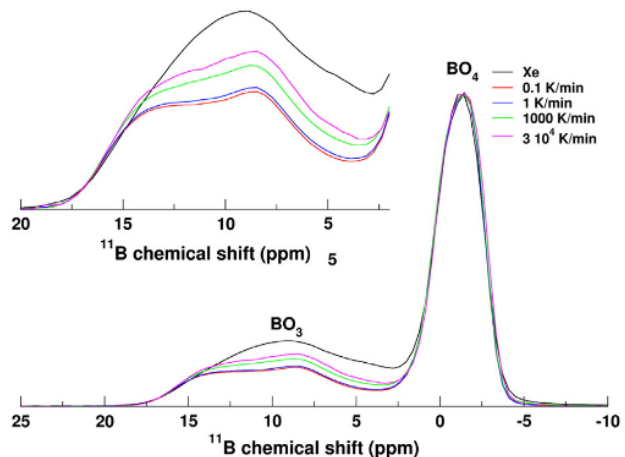

(b) ${ }^{11} \mathrm{~B}$ chemical shift (ppm) evolution for ternary NBS glass, From 83

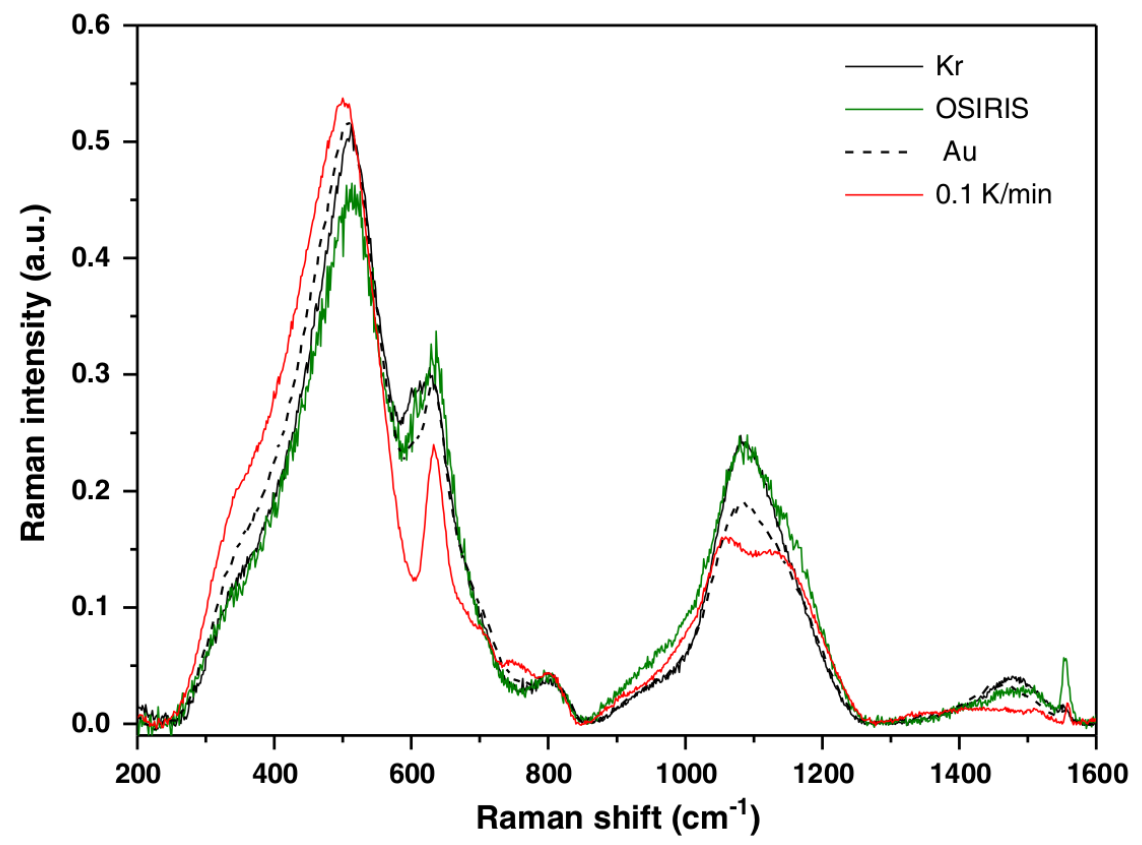

(c) Comparison between Raman spectra of the irradiated glasses with the pristine sample ("0.1 K/min"). Shift between 1000 and $1200 \mathrm{~cm}^{-1}$ shows an increase of the $\mathrm{Q}_{3}$ contribution. From 83

Figure 2.7: Structural evolution of NBS glass under irradiation 


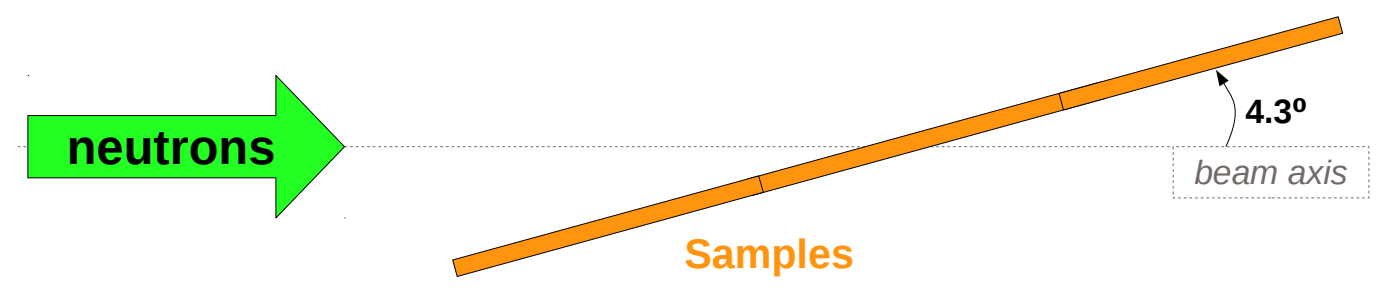

Figure 2.8: Drawing of the H112 and H21 irradiations setup, view from above.

\begin{tabular}{|c|c|c|c|}
\hline & $\begin{array}{l}\text { Beam flux } \\
\left(\mathrm{n} / \mathrm{cm}^{2} / \mathrm{s}\right)\end{array}$ & $\begin{array}{c}\text { Flux on the sample } \\
\text { surface } \\
\left(\mathrm{n} / \mathrm{cm}^{2} / \mathrm{s}\right)\end{array}$ & $\begin{array}{c}\text { Fluence on the surface } \\
\text { during } 50 \text { day reactor cycle } \\
\left(\mathrm{n} / \mathrm{cm}^{2}\right)\end{array}$ \\
\hline $\begin{array}{l}\text { H112 } \\
\text { (cold beam) }\end{array}$ & $6 \times 10^{10}$ & $4.5 \times 10^{9}$ & $2.6 \times 10^{16}$ \\
\hline $\begin{array}{l}\text { H21 } \\
\text { (thermal beam) }\end{array}$ & $1.1 \times 10^{9}$ & $8.2 \times 10^{7}$ & $4.8 \times 10^{15}$ \\
\hline
\end{tabular}

Table 2.3: Numerical values for the incident neutron capture fluxes and fluences for the H112 and H21 irradiation campaigns. Values are derived from the gold foil activation measurements. 


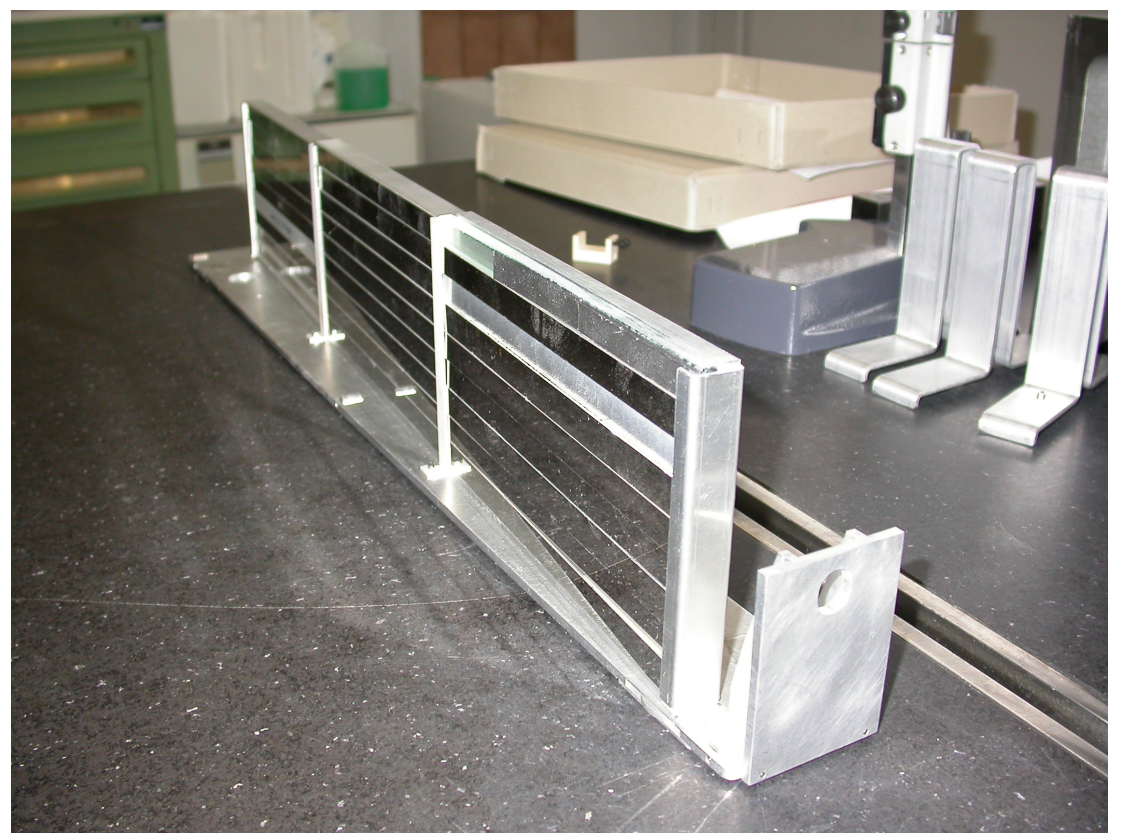

Figure 2.9: Sample holder used for irradiation at the H112 and H21 guides. View from the back. The insertion in a neutron guide is done by pushing on the vertical aluminium plate at the forefront of the picture.

The samples were made of $200 \mathrm{~mm}$ length and could be up to $15 \mathrm{~mm}$ thick. The stack height could reach $108 \mathrm{~mm}$. In total, 80 samples were irradiated for long periods (50 days minimum), a lapse of time set by the reactor cycles, and were extracted every time at shut-downs to check their surface state. Figure 2.9 shows the sample holder fully loaded with pristine samples. For each kind of glass irradiated, three surface states were prepared: a surface polished with 600 grit sandpaper, another polished up to neutron optical quality ( $<1$ micron diamond suspension), and finally a surface similar to that of a real guide that had an additional neutron reflective layer.

\subsubsection{Results}

The present study allowed us to determine a radiation hardness value for two of the four glasses, namely N-ZK7 and Borofloat. The latter showed the lowest value. For this material, due to the relatively high flux in H112, a whole reactor cycle caused a fluence large enough to splinter the sample surface, irrespective of the surface having a mirror, super-mirror, or nothing attached. The ageing experiments in H21 allowed us to find the mean radiation hardness of this material, yielding values of $2.8 \times 10^{16} \pm 1 \times 10^{16} \mathrm{n} / \mathrm{cm}^{2}$, beam flux. This value was found by the splintering of several samples around this fluence. However, one Borofloat sample broke after only two cycles in H21. This generates another radiation hardness limit that should be taken into consideration when designing a guide in Borofloat. Table 2.4 sums up the radiation limits at which the four different substrates broke or, in the case of N-BK7 and S-BSL7, the fluence limit was reached at the end of the study without any sign of splintering. It must be noted that the values of the second column, "effective flux on the sample", are the real amount of neutrons entering the glass. These results allow us to compare and rank materials in terms of resistance 


\begin{tabular}{lccc}
\hline & $\begin{array}{c}\text { Radiation limit } \\
\text { (beam fluence) } \\
\left(\mathrm{n} / \mathrm{cm}^{2}\right)\end{array}$ & $\begin{array}{c}\text { Radiation limit } \\
\text { (effective fluence on the sample) } \\
\left(\mathrm{n} / \mathrm{cm}^{2}\right)\end{array}$ & $\begin{array}{c}\text { Beam number } \\
\text { and spectrum }\end{array}$ \\
\hline Borofloat (mean value) & $2.8 \pm 1.0 \times 10^{16}$ & $2.0 \pm 0.7 \times 10^{15}$ & H21 - Thermal \\
Borofloat (lowest value) & $7.5 \pm 2.5 \times 10^{15}$ & $5.6 \pm 1.9 \times 10^{14}$ & H21 - Thermal \\
N-ZK7 & $3.7 \pm 1.3 \times 10^{17}$ & $2.8 \pm 1 \times 10^{16}$ & H112 - Cold \\
N-BK7 & $>2.0 \times 10^{18}$ & $>1.5 \times 10^{17}$ & H112 - Cold \\
S-BSL7 & $>2.0 \times 10^{18}$ & $>1.5 \times 10^{17}$ & H112 - Cold \\
\hline
\end{tabular}

Table 2.4: Beam fluence needed to splinter glass plate. Heterogeneous irradiation with a mean incidence angle of $4.3^{\circ}$.

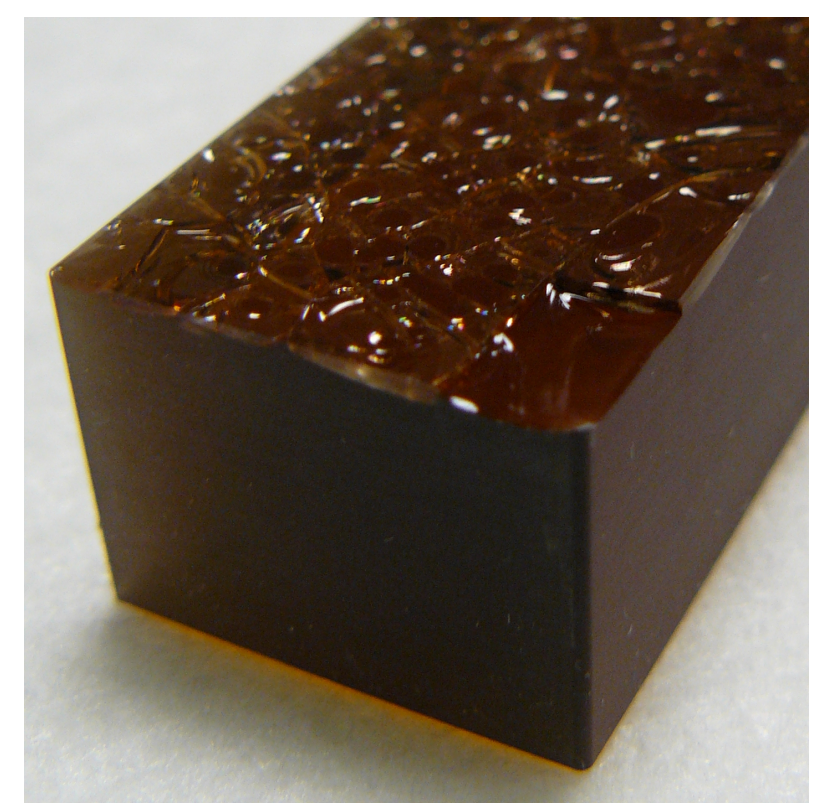

Figure 2.10: Borofloat sample irradiated in H21.

to splintering under heterogeneous neutron flux, that is to say, normal neutron guide use conditions. Figure 2.10 shows the surface state of a Borofloat sample brought above its radiation limit. The surface splintered like a mosaic and the initially transparent bulk turned brownish due to the formation of colour centres in large numbers.

Regarding the accuracy of values given in Table 2.4, we estimate that data for Borofloat and N-ZK7 are affected by some $40 \%$ uncertainty. This comes from the fact that, for a similar composition and sample preparation, all the samples did not break after the same number of reactor cycles. This highlights the variability of glass resistance to fracture and the extreme care to take when handling those results. In addition, only one sample of S-BSL7 was tested in this experiment, which make the quoted value rather uncertain. However, these fast-ageing experiments and the analysis of their results at the beginning of the thesis are to be considered as the founding elements of the work that will be presented later. 


\subsubsection{Interpretation in the light of guide irradiation ageing}

In order to understand the ageing processes undergone by neutron guides under radiation, we relied on test experiments (e.g. those carried out in H112 and H21) and then tried to relate such observations to phenomena subjected by neutron mirrors under operational conditions. The first quantity to be determined concerns the extent of the radiation damage within the glass, or more specifically, the depth down to which radiation effects may modify the glass structure. For such an avail, we have provided details pertaining to the attenuation ability of the borosilicate mirror substrate, which has been studied by means of computer simulations. Such calculations aiming to determine the neutron penetration depth have been carried out mimicking the case of a real guide, such as H522. The computational approach involves a combination of McStas 60, 109, and MCNP 64 codes. The McStas computer package is used to simulate the beam reaching the glass, whereas MCNP takes into account the propagation of neutrons within the glass substrate and simulates the ensuing nuclear reactions. The final output yields the $(\mathrm{n}, \alpha)$ reaction rate as a function of depth within the sample. The choice of H522 beam 18 to carry out such an analysis is driven by the following reasons:

- First, the H5 guide is the newest installed guide system at the ILL, and consequently it makes use of the most up-to-date neutron guide technology (coating, maximization of the source use, etc.). Hence, its geometry is probably the closest approximation to the neutron guides that will be installed in the near future.

- Second, because of its age, it should be free from the deleterious radiation effects and thus the simulation of the beam intensity can be directly related to the measured values. In contrast, an old guide may contain a number of imperfections resulting from its years of operation, which may be difficult to account for in the simulation. In fact, the beam intensity is affected not only by the guide geometry and source brightness, but also by the mirror degradation and the misalignments that appear from one guide element to the other.

The neutron losses have been simulated at four distances from the guide start. Table 2.5 summarizes the pertinent details.

The output of McStas usually consists of text files giving the neutron flux as a function of wavelength, energy, beam divergence, position in the guide, etc. For this study, two parameters were monitored:

The wavelength : from 1 to $15 \AA$, which is modelled using 57 bins of $0.25 \AA$. As MCNP operates with energy units to generate neutrons, each wavelength bin is converted into $\mathrm{MeV}$ when merging the MCNP and McStas results.

$\boldsymbol{\mu}$ : that is, the cosine of the angle subtended by the neutron trajectory and the normal to the mirror surface. This is discretized into a set of 51 bins from 0 to 0.05. Such a quantity is needed as an input parameter for MCNP when generating neutrons.

To detect the neutrons lost in a guide section, the guide element was modelled using four individual mirrors. Notice that within McStas, beam line modelling is normally performed using pre-made guide elements, with input parameters such as height and width, as well as the mirror properties. As an additional assumption, one has to note that McStas 


\begin{tabular}{c|c|c|c|c}
\hline Position & $\begin{array}{c}\text { Distance from } \\
\text { guide start }\end{array}$ & Beam neutron flux & $\begin{array}{c}\text { Neutron flux } \\
\text { penetrating in } \\
\text { substrate } \\
(\mathrm{n} / \mathrm{cm} 2 / \mathrm{s})\end{array}$ & $\begin{array}{c}\text { Reaction rate } \\
\text { reduced to } 1 / 10\end{array}$ \\
\hline 1 & $(\mathrm{~m})$ & $1.4 \times 10^{11}$ & $4.1 \times 10^{9}$ & 200 \\
2 & 0 & $4.5 \times 10^{10}$ & $2.2 \times 10^{8}$ & 100 \\
3 & 3 & $1.3 \times 10^{10}$ & $5.8 \times 10^{6}$ & 40 \\
4 & 10 & $6.8 \times 10^{9}$ & $6.0 \times 10^{5}$ & 30 \\
\hline
\end{tabular}

Table 2.5: Details about the simulated H522 guide positions. The rightmost column displays the depth at which the reaction rate reduces to one tenth of that shown at the glass surface. From 22].

treats mirrors as purely reflecting layers, devoid of any underlying substrate. A neutron transmitted (i.e. not reflected) by the mirrors can either be absorbed or transmitted through it. In our case, we considered one of the four mirrors as transmitting all crossing neutrons whereas all the others were set to capture them. A beam stop was modelled at the guide exit to absorb reflected neutrons. Finally, a large detector monitored the neutrons that had been transmitted by the only non-absorbing mirror. The component Monitor_nD was used for that purpose because of its versatility. Figure 2.11 displays the schematics of the simulated geometry.

The output file of McStas simulations consists of a text file containing several matrices arranged one below the other. The rows and columns are set as wavelength bins in rows and cosine bins in columns. The first matrix contains the flux values $(\mathrm{n} / \mathrm{s})$, the second is the error on the flux $(\mathrm{n} / \mathrm{s})$, and the last gives the number of neutrons detected at that bin.

The MCNP model of the glass mirror substrate was comprised of a $100 \mathrm{~cm}^{2}$ plate with the composition given by the chemical analysis. The source was designed as a parallel plane just above the glass surface. The source was not in contact with the glass, since this would lead to computational errors. In each simulation, neutrons were shot towards the glass surface, having a fixed energy and a given angle of incidence with respect to the surface. In total, $57 \times 51$ simulations were carried out. To measure the neutron flux at different depths from the surface, $F 1$ tallies were used. A $F M$ multiplication card computed the corresponding $(\mathrm{n}, \alpha)$ reaction rate for each flux .

The final step consisted in merging of the MCNP results and McStas matrices. This was done using the awk Linux shell command. The final output consisted in a three columned file (depth, flux or reaction rate, absolute error). The entire calculation was carried out for four distances from the H522 guide entrance, corresponding to four different regimes: guide's nose, in-pile, reactor building and guide hall. Figures $2.12 \mathrm{a}-\mathrm{b}$ display the dependence of the reaction rate versus penetration depth for the four guide locations just referenced. 


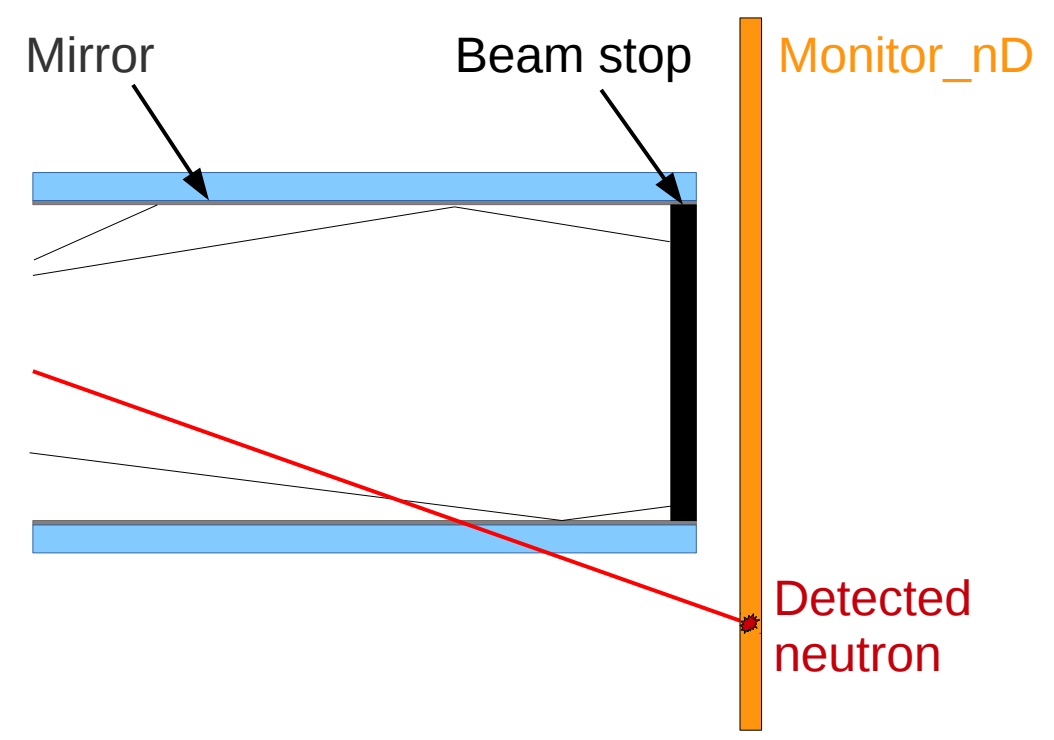

Figure 2.11: Detection of neutrons transmitted through a mirror in McStas. The bottom mirror is transmitting the non reflected neutrons, while the top one is absorbing them

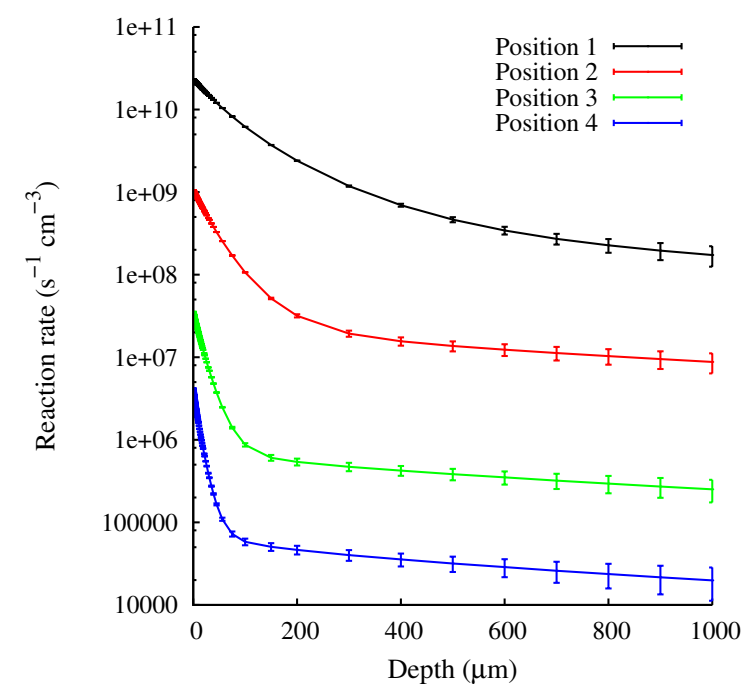

(a) Reaction rate

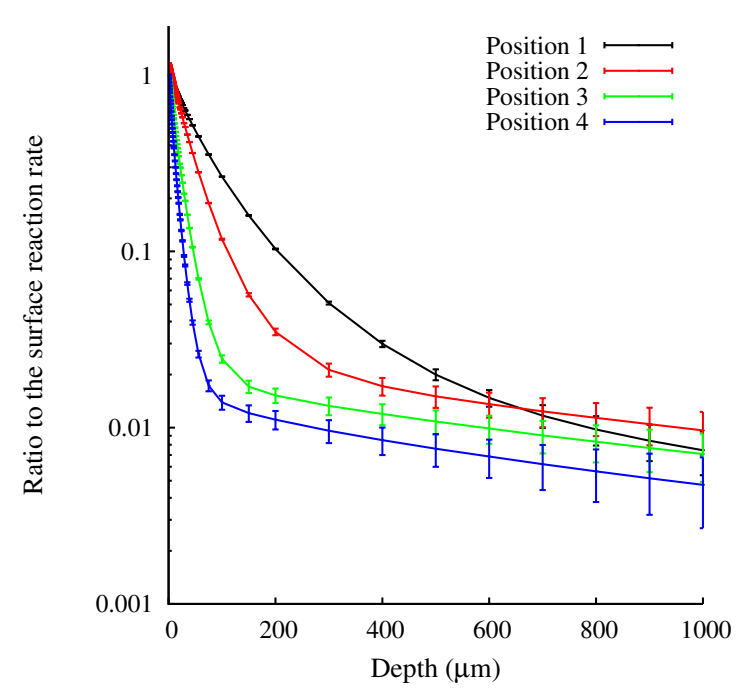

(b) Ratio

Figure 2.12: Simulation of the $(\mathrm{n}, \alpha)$ reactions as a function of the depth in the guide walls of the newly installed H522 guide. Combined McStas-MCNP simulation. 


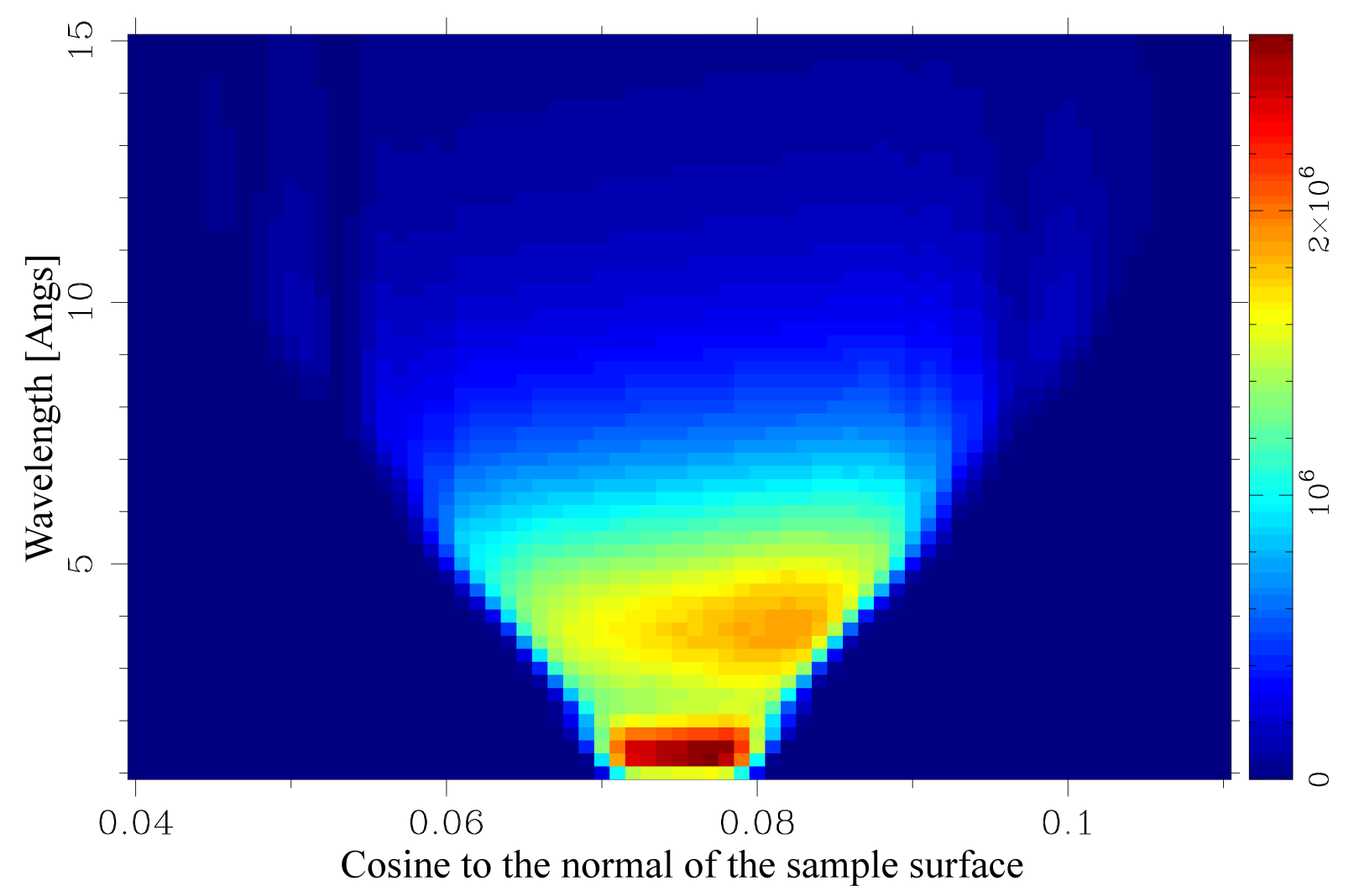

Figure 2.13: Coutour plot of the flux on samples irradiated in H112. Wavelength and angular cosine binning.

The curves plotted in Fig. $2.12 \mathrm{~b}$ show a fairly strong dependence of the reaction rate at a given depth inside the glassy substrate upon the location within the guide. The differences come from the beam energy spectrum and divergence characteristics from one position to the other. In fact, the large values shown for a location closer to the reactor core, such as positions 1 or 2 , are a direct consequence of a significantly hotter neutron energy spectrum compared to those reaching the downstream positions 3 and 4 . These positions have a larger beam divergence due to a direct view of the source. Both quantities get reduced as the beam travels along the guide path, which serves to filter out the more energetic neutrons and reduces the divergence. The strong drop in reaction rate at position 4 results from the combined effects of the decrease in beam intensity, the smaller beam divergence, as well as the colder neutron spectrum. In fact, a geometrical estimate of the direct view length $L_{\text {direct-view }}=\sqrt{8 \rho a}$ given in terms of the guide radius " $\rho$ " and width " $a$ " yields a figure which can be compared to the distance between the guide start and the fourth position. This means that, at such guide location, almost all neutrons outside of the mirror reflectivity area have been filtered out.

These results can be compared with the fast H112/H21 ageing experiment. A similar McStas $\times$ MCNP simulation was carried out in that case. Figure 2.13 displays a two dimensional density plot of the beam, giving the wavelength versus the cosine of the angle of incidence taken with respect to the normal of the sample surface. 

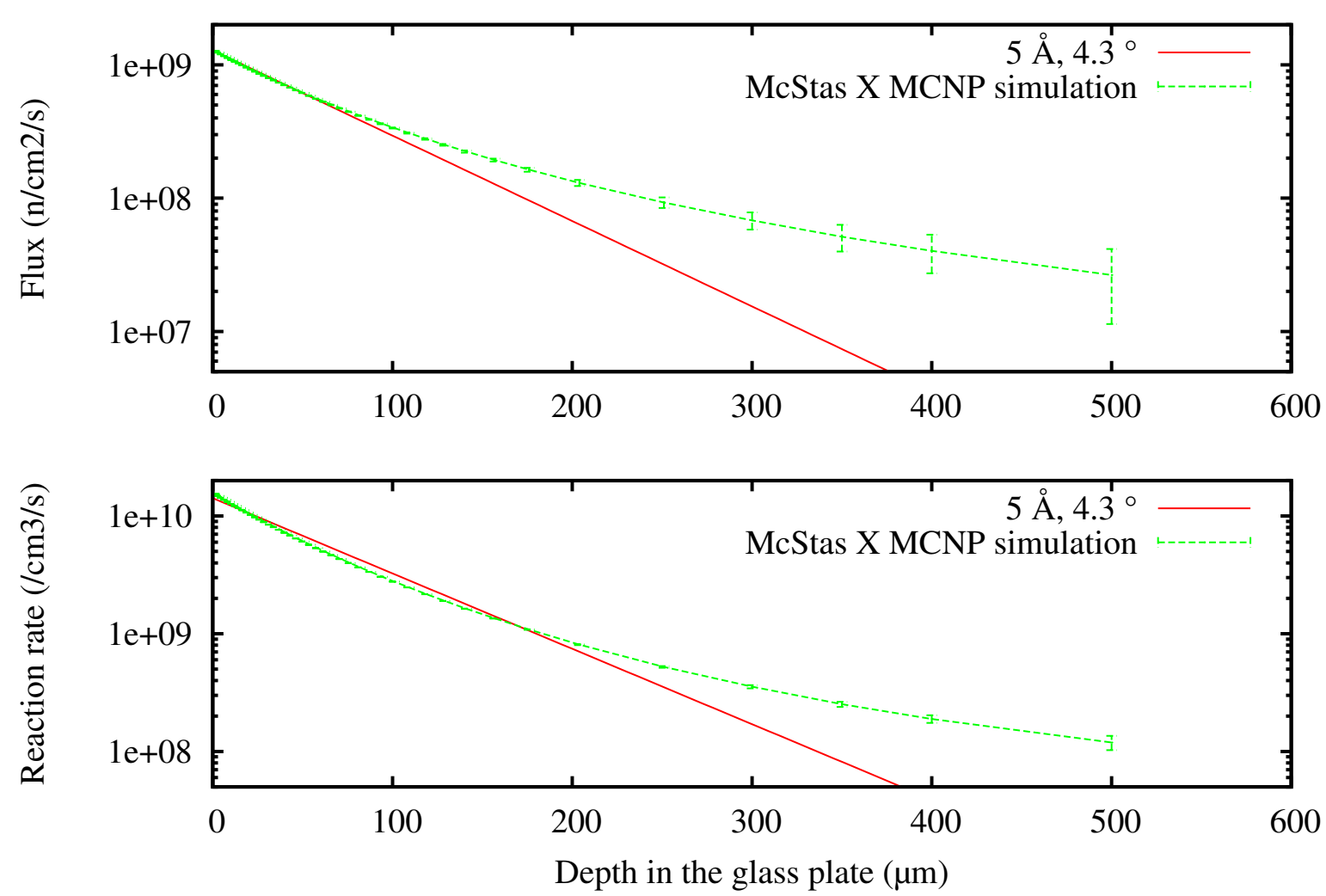

Figure 2.14: Simulation and simplified analytical calculation of the flux and the reaction rate as a function of the depth in the glass substrate irradiated in H112.

Figure 2.14 shows the final output of these simulations for Borofloat samples in the H112 settings. One can see that after $250 \mu \mathrm{m}$ of penetration, the flux and the reaction rate have been reduced by an order of magnitude. In addition, an analytical estimation assuming a monochromatic beam having an energy corresponding to where the maximum intensity appears in the McStas simulation and with an angle of incidence of $4.3^{\circ}$, is added to the last plots. Similar simulations have been done for the other glasses. As they did not show significant differences, the corresponding plots are not drawn here.

To give a numerical comparison between the H112 experiment and a real guide, one should bear in mind that the depth at which the reaction rate becomes $1 / 10$ of that at the glass surface becomes about twice that value at position 2 listed in Table 2.5. Notice, however, that such figures are small with respect to the glass plate thickness, usually from 9 to $15 \mathrm{~mm}$. This irradiation experiment, then, has conditions very close to those characteristic of real guide ageing. This consolidates the values given in Table 2.3 (p 37) as landmarks to select these materials when building new beam lines. By combining the values of H112/H21 experiments and H522 simulations detailed here, the lifetime expectancy of the different glasses have been inferred and are reported in Table 2.6. From the table, one can see that Borofloat is not at all adapted for installation in the first meters of a guide; a real-world example if the incident observed in 1978 with Pyrex guides, a material rather similar to Borofloat. Considering the error margins, the installation of Borofloat even $10 \mathrm{~m}$ from the source must be carefully considered because it might 


\begin{tabular}{cccccc}
\hline & Flux cracking limit & \multicolumn{4}{c}{ Reactor cycles needed to splinter } \\
& $\left(\mathrm{n} / \mathrm{cm}^{2}\right)$ & Position 1 & Position 2 & Position 3 & Position 4 \\
\hline Borofloat (mean value) & $2.0 \pm 0.7 \times 10^{15}$ & 0.1 & $2.2 \pm 0.8$ & $84 \pm 30$ & $810 \pm 290$ \\
Borofloat (lowest value) & $5.6 \pm 1.9 \times 10^{14}$ & 0.03 & 0.6 & $22 \pm 8$ & $200 \pm 68$ \\
N-ZK7 & $8.4 \pm 3 \times 10^{15}$ & $1.6 \pm 0.6$ & $30 \pm 10$ & $1100 \pm 390$ & $11000 \pm 3800$ \\
N-BK7 & $>6.0 \times 10^{17}$ & $>8.5$ & $>48$ & $>1800$ & $>17000$ \\
\hline
\end{tabular}

Table 2.6: Thermal neutron fluence cracking limit found during the H112/H21 experiments and corresponding lifetime expectancy when used as guide mirrors.

need to be replaced after only 10 years of use. Also, installation of N-ZK7 should be forbidden within the first meters of a guide; however for distances of $10 \mathrm{~m}$ or more, the mechanical failure limit is large enough compared to the lifetime of a beam line. Finally, N-BK7 appears to be resistant enough to be installed in-pile for at least 10 years. These considerations do not take into account the additional stresses on guide walls when they are not installed in evacuated housing. The splintering limit would probably be lower in such a case.

\subsection{Ageing mechanism}

The combination of the data presented in this chapter:

- Neutron guide failure incidents,

- Glass structure and behaviour under irradiation literature,

- and H112/H21 fast-ageing results

allowed us to draw a global mechanism describing how the transport of slow neutrons in a glass guide may induce its brittle rupture. This description, reported in Fig. 2.15, is an attempt to link the material intrinsic characteristics (chemical composition, internal structure, etc.) with its evolution under neutron flux and potentially its rupture. The work presented in this document tried to explore and clarify every one of the elements of this diagram. 


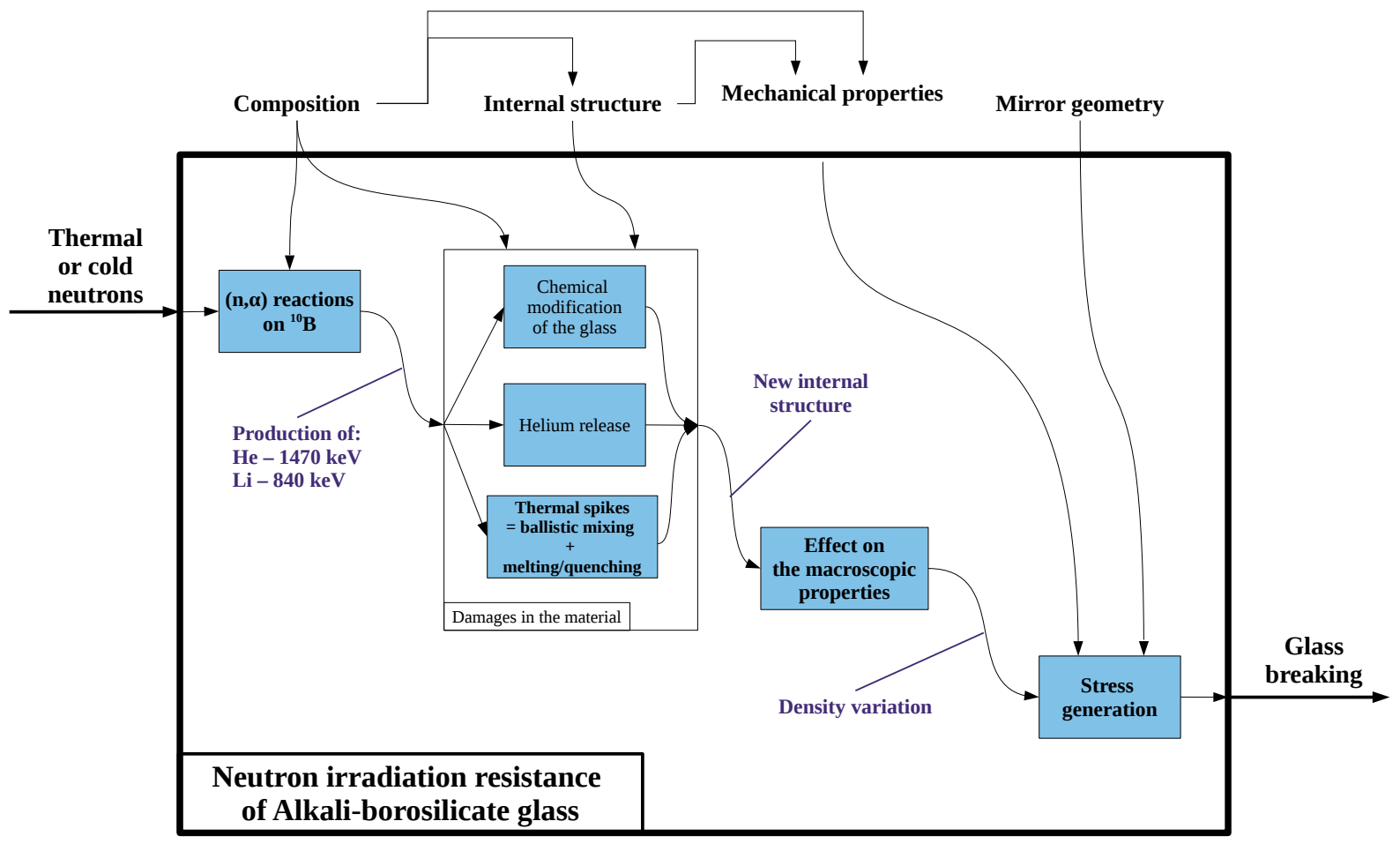

Figure 2.15: Neutron irradiation ageing mechanism of Alkali-borosilicate glass plate when used for beam guide manufacturing. 


\section{Chapter 3}

\section{Macroscopic characterization and irradiation program}

\subsection{Differential scanning calorimetry}

\subsubsection{Principle and experimental procedure}

The signatures of the glass-liquid transition region of a vitreous material provide us with information of its thermal history, a matter as relevant as its chemical composition as far as the internal structure of the material after the thermal quench is concerned. The $\mathrm{T}_{\mathrm{g}}$, or mean transition temperature, of the different glasses studied is listed in Table 3.1. and correspond to values taken from the data sheets published by the manufacturers on their websites. From data given in the table just referenced we can make two relevant comments which highlight the complex chemistry of these glasses:

- First, the received wisdom tells us that $\mathrm{T}_{\mathrm{g}}$ should be inversely proportional to the network modifiers' content. The numbers quoted above show that such a view does not reliably describe the current data, since Borofloat, which has the smallest amount of alkalis, also has the lowest $\mathrm{T}_{\mathrm{g}}$.

- Borofloat, which is manufactured by the fastest quench process compared to the three other optical glasses, has the lowest glass transition temperature.

It is also worth remarking that knowledge of the glass transition temperature also provides an indirect tool to detect the existence of separated phases. Studies of Polyakova 85 and Roderick et al. 91] have shown that an unmixed (i.e partially segregated) glass may exhibit one $\mathrm{T}_{\mathrm{g}}$ per phase, provided that the glass transition phenomena are distinct enough to be detected by the measuring system, there are no partial crystallization processes ongoing across the transition region, and the transition temperatures are well

\begin{tabular}{ccccc}
\hline & Borofloat & N-ZK7 & N-BK7 & S-BSL7 \\
$\mathrm{T}_{\mathrm{g}}\left({ }^{\circ} \mathrm{C}\right)$ & 525 & 539 & 557 & 576 \\
\hline
\end{tabular}

Table 3.1: Glass transition temperatures published by the manufacturers. 
separated.

Differential Scanning Calorimetry (DSC) analysis has thus been used to investigate the thermal behaviour of the material. The technique is based upon heating a sample at a constant rate and monitoring the heat absorbed or released by the sample compared to a reference. For such a purpose, the sample is put within a crucible, while the reference is an empty crucible which is supposed to have the same shape, composition, and weight. In our case, the crucibles are made out of alumina $\left(\mathrm{Al}_{2} \mathrm{O}_{3}\right)$ in order to reach temperatures above $\mathrm{T}_{\mathrm{g}}$. The machine was a $D S C 131$ from Setaram Instrumentation. Its maximum operating temperature is $700{ }^{\circ} \mathrm{C}$, which is high enough to investigate the glass transition temperature of our materials.

Several measurements were made to optimize the experimental parameters. The sample weight was chosen from $5 \mathrm{mg}$ to $50 \mathrm{mg}$, and the temperature ramp was varied from $1 \mathrm{~K} / \mathrm{min}$ to $10 \mathrm{~K} / \mathrm{min}$. Also, as the glass transition is a weak phenomenon if compared to the strong peak signalling of crystal melting, blank measurements using an empty crucible as a reference were made to account for heat deviation. Such phenomena arise from the small differences existing between the two crucibles and the furnace itself.

The final measurements shown here were done consecutively without shutting down the machine or modifying the $\mathrm{N}_{2}$ input pressure. Several blank heating and cooling ramps were carried out to stabilize the machine for the real measurements, as well as records of the empty cell signal as mentioned earlier. Such a procedure allowed us to ensure the reproducibility of the measurements. The experiments were carried out with around $50 \mathrm{mg}$ of powder to maximize the glass transition signal. The sample was heated at $20 \mathrm{~K} / \mathrm{min}$ up to $450{ }^{\circ} \mathrm{C}$, and then at $5 \mathrm{~K} / \min$ up to $650{ }^{\circ} \mathrm{C}$.

\subsubsection{Results}

Figure 3.1a shows the five heating ramps at the beginning of the batch of measurements. One can see that the first ramp stands apart from the four others. This is probably due to the fact that it was the first time the machine reached such temperature since it was turned on. Therefore, a baseline signal was determined by averaging over the four last ramps only. The same experiment was done once all the glasses had been measured and gave similar curves to the previous experiment.

Two examples of the data treatment are given in Fig. 3.1b and 3.1c for Borofloat and N-ZK7, respectively. On each plot one can see the raw signal (plain line), after subtraction of the baseline (in dotted line), and after correction of the slope (dash-dotted line). This last step is achieved by fitting the slope between $480{ }^{\circ} \mathrm{C}$ and $520{ }^{\circ} \mathrm{C}$ and removing it from the curve. Doing this allows us to better see the glass transition signal. Finally, Fig 3.1d shows the DSC curves of the four glasses after their baseline and slope correction.

It appears that N-ZK7, N-BK7, and S-BSL7 glass transition phenomena can be easily detected thanks to the strong peak located around $545{ }^{\circ} \mathrm{C}$ for $\mathrm{N}-\mathrm{ZK} 7$ and $591{ }^{\circ} \mathrm{C}$ for the two others. As explained by Rault [90], such a feature is caused by glass ageing. This feature is not surprising for these glasses as they are maintained at a temperature near $\mathrm{T}_{\mathrm{g}}$ after solidification to release internal stresses and avoid any birefringence. Let us 


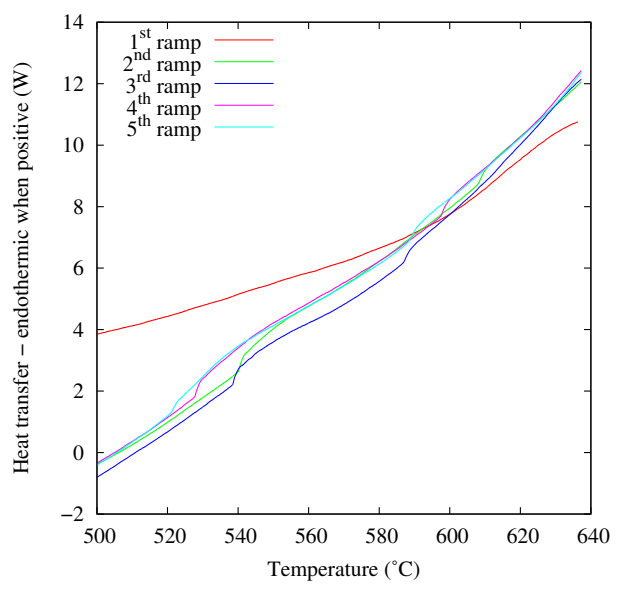

(a) Empty alumina crucible

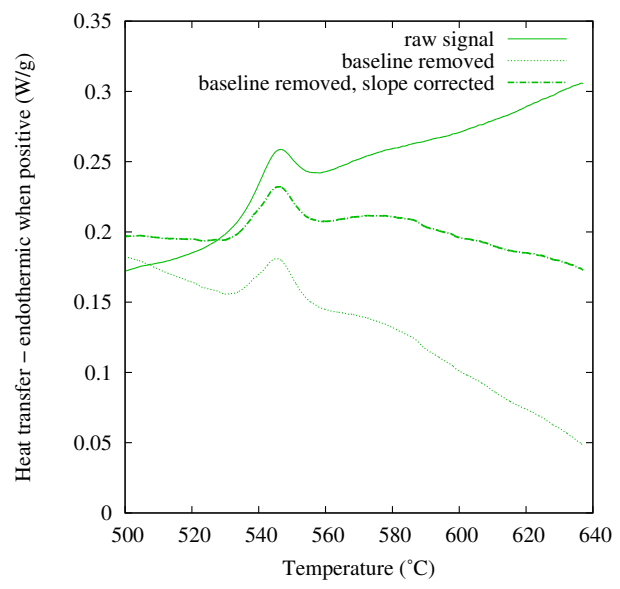

(c) N-ZK7

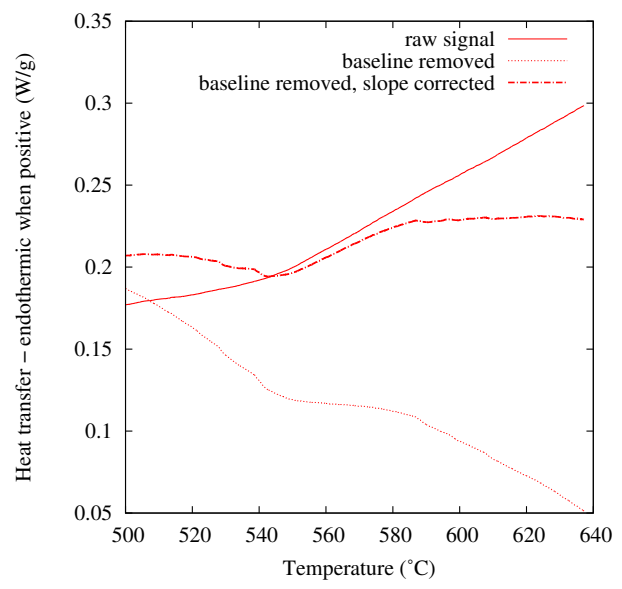

(b) Borofloat

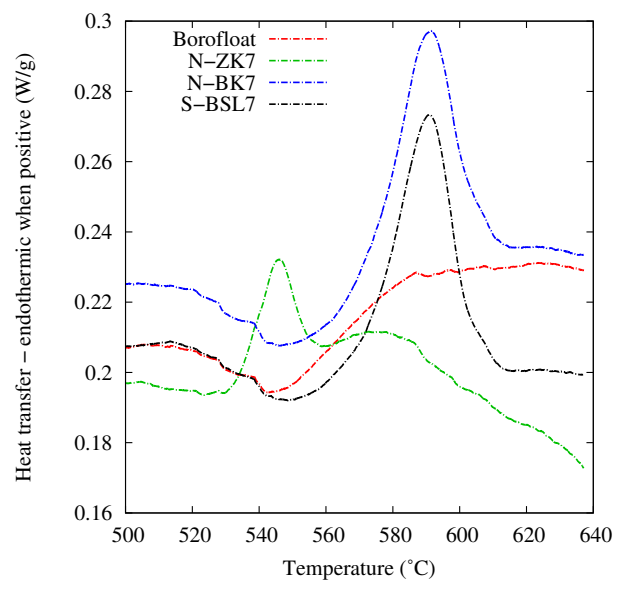

(d) Corrected signal of the 4 glasses

Figure 3.1: DSC signals for different configurations.

recall that those glasses are normally manufactured for light optics applications. On the contrary, Borofloat shows a glass transition phenomena that starts around $540{ }^{\circ} \mathrm{C}$ without ageing. It must be noted that the glass transition temperature of the different materials does not accurately match the values quoted by the manufacturers; it is determined as the temperature at which the heat transfer slope becomes positive. N-BK7 and S-BSL7 yield the same value around $550{ }^{\circ} \mathrm{C}, \mathrm{N}-\mathrm{ZK} 7$ around $530{ }^{\circ} \mathrm{C}$, and Borofloat around $545{ }^{\circ} \mathrm{C}$. The difference from the tabulated values might be explained by the post solidification thermal history of the glasses, as well as due to the relatively large amount of powder used for the experiment which was needed to measure clear signal.

This experiment was originally done to detect phase separation in the materials. Considering the curves reported in Fig. $3.1 \mathrm{~d}$, one cannot infer that any double glass transition happens for any of the materials. As explained before, in the case of a glass with two phases, their detection by DSC needs to fulfil many conditions. Further phase separation analysis will be discussed later in the document, specifically with TEM, Raman, and SANS. 


\begin{tabular}{ccccc}
\hline & Borofloat & N-ZK7 & N-BK7 & S-BSL7 \\
\hline Density $\left(\mathrm{g} / \mathrm{cm}^{3}\right)$ & 2.23 & 2.49 & 2.51 & - \\
$\mathrm{E}\left(10^{3} \mathrm{~N} / \mathrm{mm}^{2}\right)$ & 64 & 70 & 82 & 80 \\
$\mu$ & 0.2 & 0.214 & 0.206 & 0.205 \\
$\mathrm{HK}_{0.1 / 20}$ & 480 & 530 & 610 & 570 \\
$\mathrm{~K}_{\mathrm{IC}}\left(\mathrm{MPa} / \mathrm{m}^{1 / 2}\right)$ & - & - & 1.1 & - \\
\hline
\end{tabular}

Table 3.2: Mechanical properties of glasses provided by manufacturers 3, 4.

\subsection{Hardness and fracture toughness}

One of the main aims of the present $\mathrm{Ph}$.D. thesis is to contribute towards the understanding of why some glasses crack under neutron flux. As a prerequisite, a decent knowledge of their mechanical properties prior to irradiation is a must. Most of the relevant values, such as the density, Young's modulus (E), Poisson ratio $(\mu)$, Knoop hardness $\left(\mathrm{HK}_{0.1 / 20}\right)$, and optical coefficients are published by the glass manufacturers on their websites. Here we reproduce such values, which are given in Table 3.2. Notice that only a single fracture toughness $\left(\mathrm{K}_{\mathrm{IC}}\right)$ value has been found in the literature for the glasses we are concerned with. This is not surprising, as N-ZK7, N-BK7, and S-BSL7 are optical materials used for their light transmission properties. In contrast, the Borofloat surface resistance was investigated using techniques such as the scanning-scratch-test.

The established way to investigate the fracture toughness relies in the three point bending test. In this study, and because the available material quantities were not large, we have decided to use the Vickers indentation technique [5,117]. This method allows us to access another measure for the material hardness which can be used to yield the fracture toughness. The measurement procedure consists in loading the material with stresses, which are then allowed to relax by means of crack formation. This shares some characteristics with the observed surface splintering of the irradiated mirrors.

\subsubsection{Experimental setting}

The equipment used is a Buehler micro-indenter (MICROMET 5104) equipped with a Vickers diamond head. The Vickers hardness (HV) value is defined as the ratio of the force applied via the diamond tip ( $F$ in kgf units) by the surface of the pyramidal impression $\left(A\right.$ in $\left.\mathrm{mm}^{2}\right)$. This area can be derived by measuring the diagonals of the square trace seen when observing the indentation with a microscope,

$$
\begin{gathered}
H V=\frac{F}{A} \\
A=\frac{\langle d\rangle^{2}}{2 \sin \left(\frac{136^{\circ}}{2}\right)},
\end{gathered}
$$

where $\langle d\rangle$ stands for the average value of the two diagonals. The thickness of the samples was the same compared to those used for neutron guide mirror plates, which is 9 or $10 \mathrm{~mm}$ 


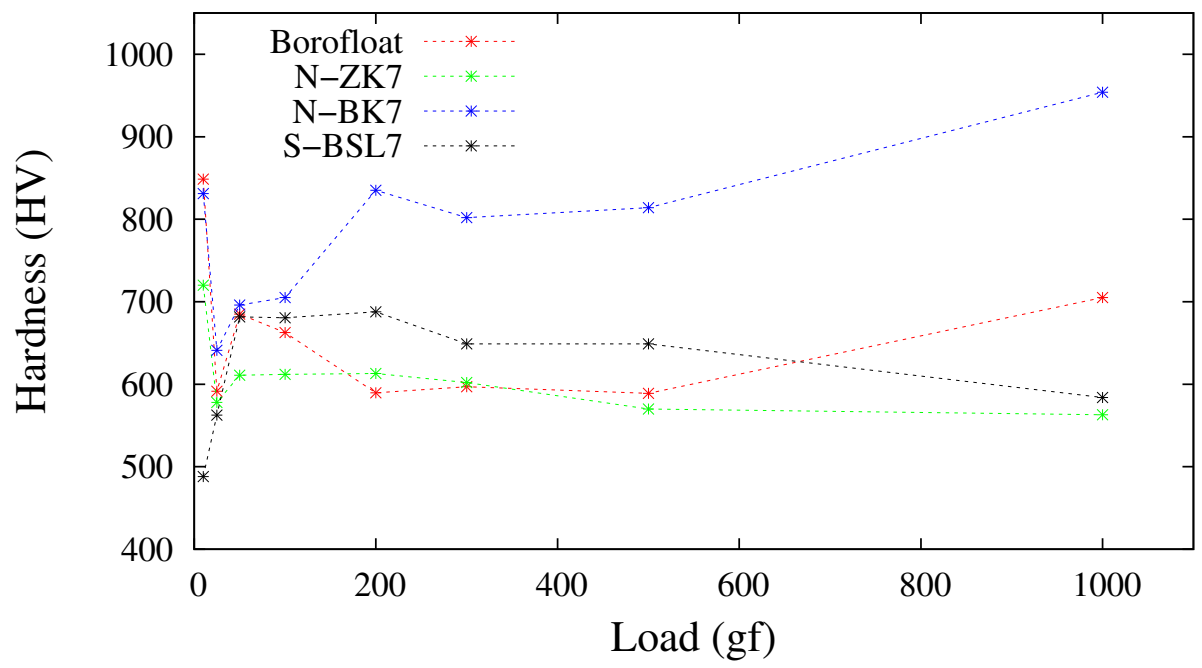

Figure 3.2: Vicker's hardness measured on pristine glasses. Lines were drawn between the points to ease the reading.

depending on the glass type. Indentations were done with varying duration from $10 \mathrm{~s}$ to $60 \mathrm{~s}$ and the load from 10 to $1000 \mathrm{~g}$.

\subsubsection{Results}

Let us first state that the HV values did not show any clear correlation with the loading time. In consequence, the results described hereafter correspond to loading times of $30 \mathrm{~s}$, which correspond to typical values found in the literature [29,65]. The evolution of HV as a function of the indentation force is displayed in Fig 3.2 . One can see that the four glasses show strong variations at light loading $(<200 \mathrm{gf})$, getting some stabilization afterwards, at least until 500 gf. The force reference has been taken to 300 gf, something in agreement with the study from Clément et al. [29]. The averaging of 4 indentations with $300 \mathrm{gf}$ loads yielded HV values of $597 \pm 6$ for Borofloat, $600 \pm 7$ for N-ZK7, $674 \pm 17$ for N-BK7, and $649 \pm 6$ for S-BSL7. Even though the manufacturers did not give error margins on the published Knoop hardness, the glasses are ranked similarly with both hardness techniques.

Because of the shape of the Vickers indenter, cracks often initiate at the pyramid tips. The present method has thus been adapted to determine the fracture toughness $\left(\mathrm{K}_{\mathrm{IC}}\right)$ by measuring the crack length. The first result we can mention is that only N-BK7 and S-BSL7 generated cracks with the indentation. This occurred at the $25 \mathrm{mg}$ dose and the crack length increased with the loading force. In the case of Borofloat and N-ZK7, no cracks were generated at the pyramid tips, even at $1 \mathrm{kgf}$. These materials do also splinter, but the shape of such cracks follow arcs along the mark. The study from Clément et al. has compared formulas from different authors deriving $\mathrm{K}_{\mathrm{IC}}$ from crack lengths to threepoints bending test results. We applied three of those formulas to find an approximate fracture toughness for N-BK7 and S-BSL7. From Blendell's work, [20],

$$
K_{\mathrm{IC}}=0.0303\left(H_{v} a^{1 / 2}\right)\left(E / H_{v}\right)^{2 / 5} \log (8.4 a / c)
$$




\begin{tabular}{ccc}
\hline & N-BK7 & S-BSL7 \\
\hline Blendell & 0.92 & 0.89 \\
Lankford & 0.95 & 0.90 \\
Liang et al. & 0.77 & 0.74 \\
\hline
\end{tabular}

Table 3.3: $\mathrm{K}_{\mathrm{IC}}$ in $\mathrm{MPa} / \mathrm{m}^{1 / 2}$ for $\mathrm{N}-\mathrm{BK} 7$ and S-BSL7.

from Liang et al.,

$$
K_{\mathrm{IC}}=0.0495\left(H_{v} a^{1 / 2}\right)\left(E / H_{v}\right)^{2 / 5}(a / c)^{(c / 18 a)-1.51}
$$

and from Lankford,

$$
K_{\mathrm{IC}}=0.0782\left(H_{v} a^{1 / 2}\right)\left(E / H_{v}\right)^{2 / 5}(a / c)^{-1.56}
$$

where $a$ is the half diagonal length from the track center and $c$ is the crack length $+a$. To have $K_{\mathrm{IC}}$ in $\mathrm{MPa} / \mathrm{m}^{1 / 2}, a$ and $c$ must be in $\mathrm{mm}, E$ in GPa, and $H V$ in GPa. Once computed, they give the values reported in Table 3.3. Compared to the value found for N-BK7 in the literature, the values are not fully in agreement, especially for the Liang et al. model. However, the Lankford formula provides a relatively good agreement if compared to $1.1 \mathrm{MPa} / \mathrm{m}^{1 / 2}$.

These tests have shown that differences in neutron radiation resistance between the glasses cannot be explained with the fact that some of them are naturally more prompt to fracture than others. Indeed, N-BK7 and S-BSL7, which are the most radiation resistant, are precisely those which fracture first due to Vickers indentation. The absence of crack generation at the tips for Borofloat and N-ZK7 might be explained by a compaction of their internal structure. Silica for instance, because of its relatively "hollow" structure, is prompt to compact under compression [34]. In particular, Borofloat has a high content of $\mathrm{SiO}_{2}(82 \%)$ compared to N-BK7 or S-BSL7 (72.7\% and $73 \%$ ). This assumption will be discussed later thanks to the analysis of structure of the glasses.

\subsection{Electronic Microscopy Imaging}

According to [87, the existence of a segregated phase in a glass influences its behaviour under irradiation. In our case, the ballistic mixing generated by the $(\mathrm{n}, \alpha)$ reactions might blend the existing domains and create a new one with new properties. In consequence, this internal process would possibly be at the origin of a global modification of the material and lead to the generation of cracks. The detection of different phases can be directly achieved by transmission electron microscopy (TEM) imaging. Indeed, the incident electron beam should be sensitive enough to the electronic density of the material that it crosses and may lead to the detection of de-mixing phenomena, provided that the contrast between the different phases is large enough.

The segregation in alkali-borosilicate glasses $(\mathrm{ABS})$ usually takes place leading to a borate-rich and a silica-rich phase [48. The challenge here stands from the small 
electronic contrast between the two domains which is low and thus makes it difficult to detect such phase separation by electronic imaging. The procedure followed here first involved imaging by electronic transmission of the glass without special preparation. A chemical etching with hydrofluoric acid was then carried out to dissolve any hypothetical silica-rich phases and keep the borate-rich ones.

The different electronic imaging measurements have been done on a JEOL Transmission Electronic Microscope, model LaB6 JEM-2010, with a 200kV accelerating tension. A diamond tip has been used to scratch glass specimens in order to generate samples with a thickness below 100nm at the tips. This technique has the advantage of preserving the internal structure of the glass when compared to other methods such as the Focused Ion Beam milling.

\subsubsection{TEM observation}

Pictures of TEM images for the four studied glasses are shown in Fig. 3.3, where the glass fragments are shown by the darker areas within those images. A look at the figures shows that on the grounds of the homogeneity of the glass images, one can classify the four materials into two groups, with Borofloat, N-BK7, and S-BSL7 on one side and NZK7 on the other.

The pictures for the former group tell us that there should not be any significant segregation within those materials. Notice that the shades having a typical dimension of $5 \mathrm{~nm}$ in Fig. 3.3 a-b most probably arise from oscillations of sample thickness that smoothly increase while get further from the edge. Let us recall that, as mentioned above, these observations do not prove that there is no phase separation within glasses like Borofloat, since the electronic contrast between boron and silicon rich areas is low. In contrast Fig $3.3 \mathrm{~b}$ featuring N-ZK7 clearly shows darker dots with a typical size around $5 \mathrm{~nm}$, indicative of demixing of the glass with the creation of a domain with higher electronic density. If we look at the N-ZK7's composition, Table 2.1 p.24, we can see that the amount of zinc oxide is around $10 \%$. One could conclude that these dark areas could be $\mathrm{Zn}$ rich. This idea has been tested by TEM-FEG observations and is described further in the document.

\subsubsection{Chemical etching for TEM imaging}

Chemical etching with $10 \%$ hydrofluoric acid solution was carried out for the four glasses, aiming to dissolve the silicon-rich phase and leave the alkali-boron-rich one. The chemical process was done on samples polished to neutron reflection optical quality. No matter how long the immersion time within the acid solution lasted, no additional contrast was observed with TEM.

\subsubsection{STEM chemical analysis}

As a further characterization step, a set of measurements using a Scanning Transmission Electron Microscope (STEM) on the glass showing significant structure on TEM (in our case N-ZK7) were carried out. The observations were performed using a MET Jeol 2100 F- $200 \mathrm{kV}$ FEG machine. Thin samples were produced by scratching the polished surface 


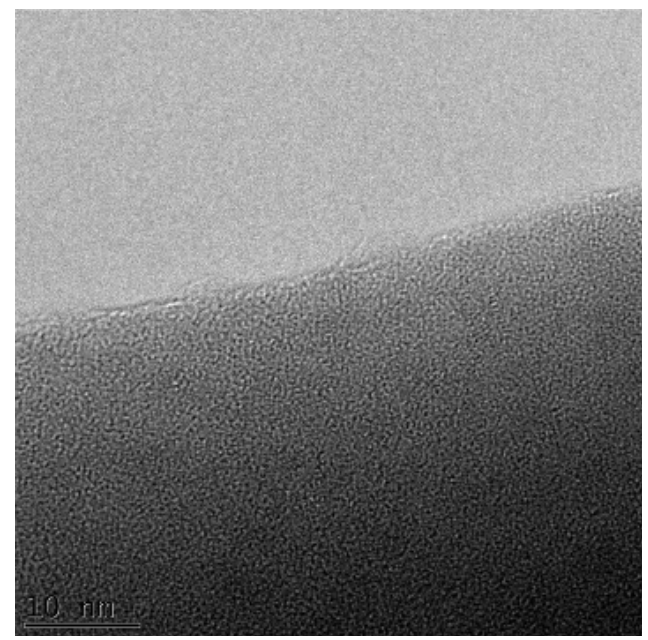

(a) Borofloat

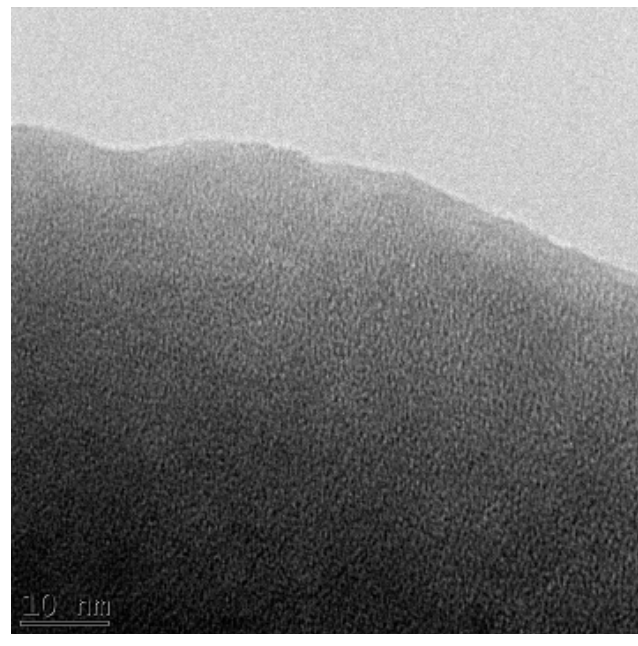

(c) N-BK7

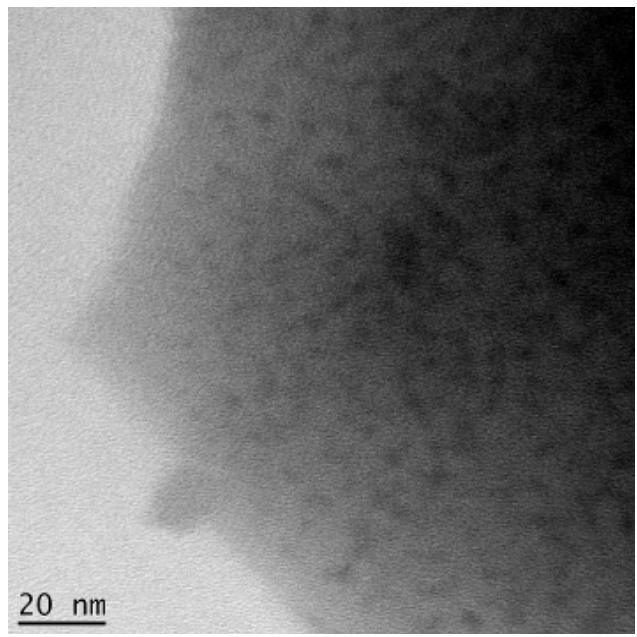

(b) N-ZK7

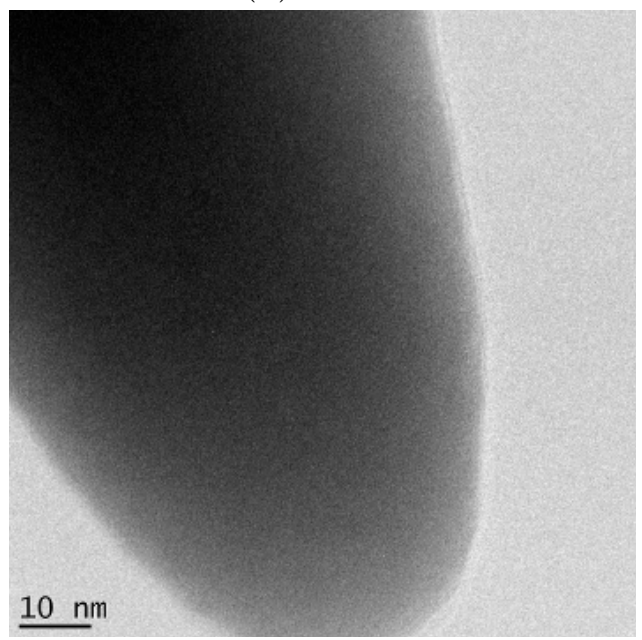

(d) S-BSL7

Figure 3.3: TEM imaging of different glass types. 
of $1 \mathrm{~cm}$ thick pieces, and the analysis focused on the narrow edges of the splinters. The recorded STEM High-Angle Annular Dark Field (HAADF) images are shown in Fig 3.4. Pictures (b)-(f) within the figure display maps for silicon, boron, zinc, aluminium, and sodium, respectively. These are a result of the use of the HAADF technique of TEM imaging, which is based upon the detection of the transmitted electrons that are strongly scattered by the sample. In fact, the deviation from normal incidence of the electron beam upon the sample can be correlated with the atomic number of atoms within the materials, and therefore the technique enables us to resolve the individual atomic species.

Contrary to the colour coding in the TEM images, the white (high intensity) areas in a HAADF image such as those shown in the (a) frame of Fig. 3.4a correspond to domains with a high electronic density. Therefore, such whitish areas here correspond to the dark patches of the TEM images.

The analysis of the STEM-HAADF images, once resolved in terms of increasing Z material components, gives us a clue on the origin of the heterogeneities shown in TEM pictures. In fact, a glance to Fig 3.4d shows that the zinc rich areas in Fig. 3.3b correspond to the white spots in the HAADF image. The concentration of the different elements as a function of the horizontal position was extracted form their respective mapping with ImageJ software. The technique consisted in integrating the light intensity in a $20 \mathrm{~nm}$ height band vertically centred. The results are gathered in Fig.3.5. The distribution of zinc heterogeneities is strongly correlated with those shown for the sodium concentration, and to a lesser extent with those for aluminium. In contrast, the less marked patterns observed for Si and B cannot be clearly correlated with the pattern shown in Fig. 3.4a. One could estimate that there is a very slight depletion in silicon but it is not entirely obvious.

As a result derived from inspection of the images in Fig. 3.4, we can picture N-ZK7 as having a fairly homogeneous borosilicate network with clusters of aluminium and zinc oxides. The strong concentration of sodium in those clusters suggests that this alkali goes preferentially there to charge compensate zinc and aluminium to convert them into network formers. Notice, however, that the sodium content of this glass is not large enough to convert the full $\mathrm{Al} / \mathrm{Zn}$ population into network formers.

Calcium mapping has also been done, but is not shown here as the concentration in this element is too weak to formulate any conclusion concerning its segregation or lack thereof.

\subsection{A new fast homogeneous irradiation equipment}

It goes without saying that to study the evolution of a material under neutron flux, one needs to have access to an irradiation setup adapted to the phenomena of interest. Prior to the development of the current Ph.D. thesis, the ILL had generated a good quantity of borosilicate glass samples heterogeneously irradiated either during the H112/H21 test campaigns (see section 2.6 $\mathrm{p} 35$ ) or taken from decommissioned beam lines. Such samples were mostly composed by Borofloat, N-ZK7, and N-BK7 glasses and consist in thick plates which were irradiated from one side at grazing incidence. These irradiation conditions lead to samples where the ageing is strongly dependent on the depth within the plates and makes it a challenge to the study of bulk properties' response to neutron flux. To overcome such a difficulty, a new irradiation apparatus that would allow thermal neutron 


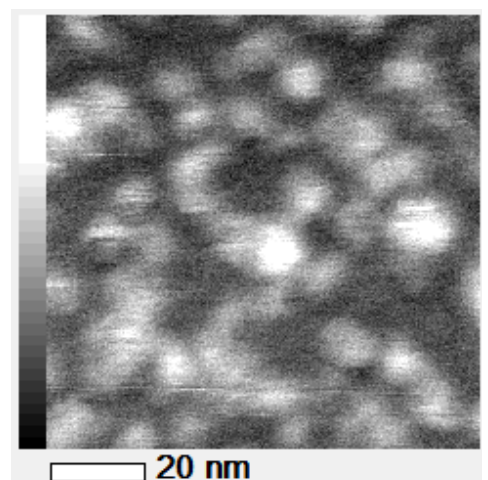

(a) STEM-HAADF image, white areas have heavier elements

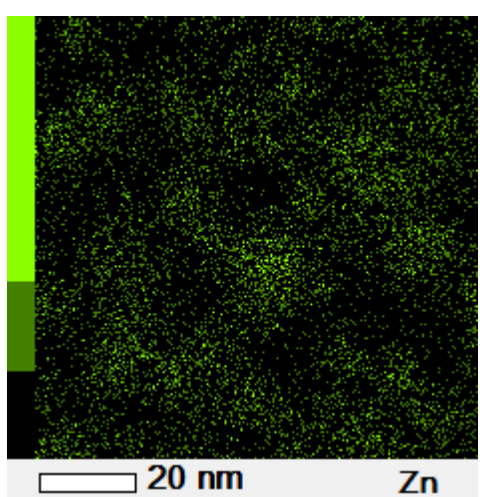

(d) Zn mapping

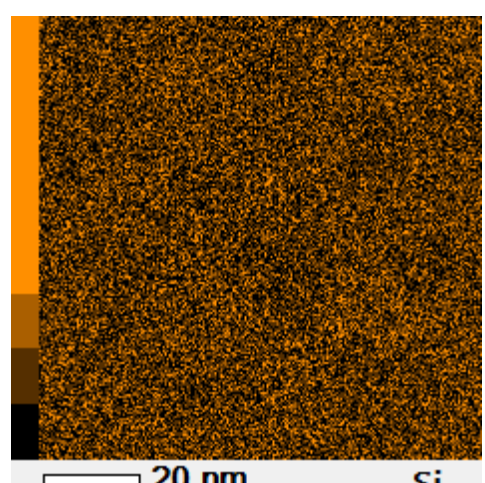

$20 \mathrm{~nm}$

(b) Si mapping

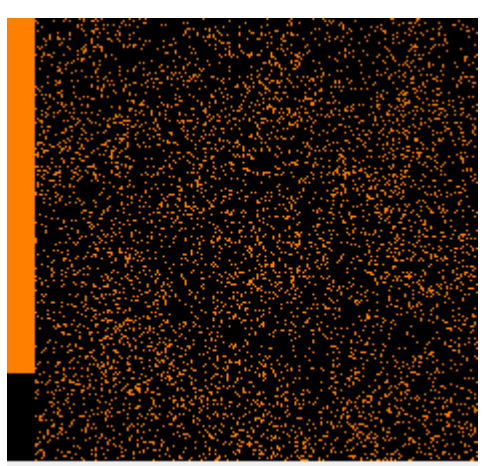

$20 \mathrm{~nm}$

(e) Al mapping

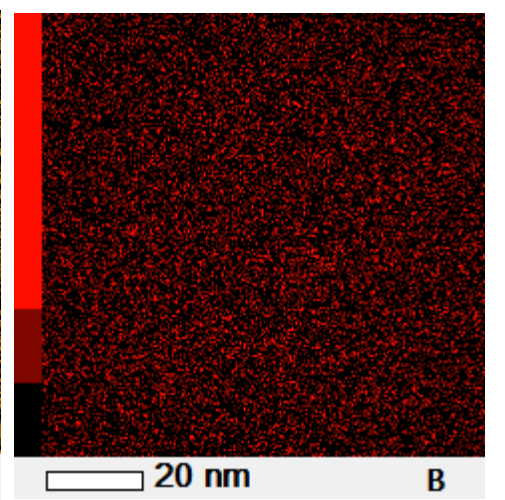

(c) B mapping

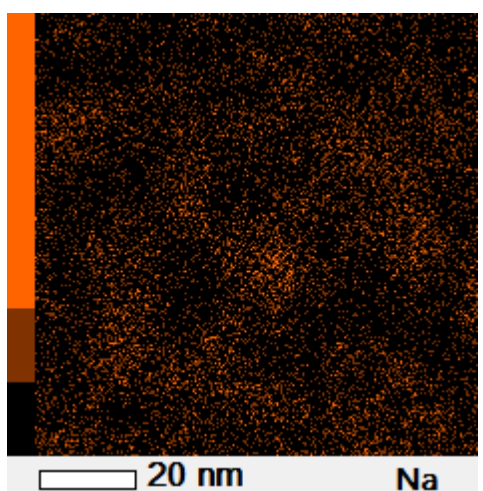

(f) Na mapping

Figure 3.4: STEM-HAADF imaging of N-ZK7 and element mapping of Si, B, Zn, Al, Na.

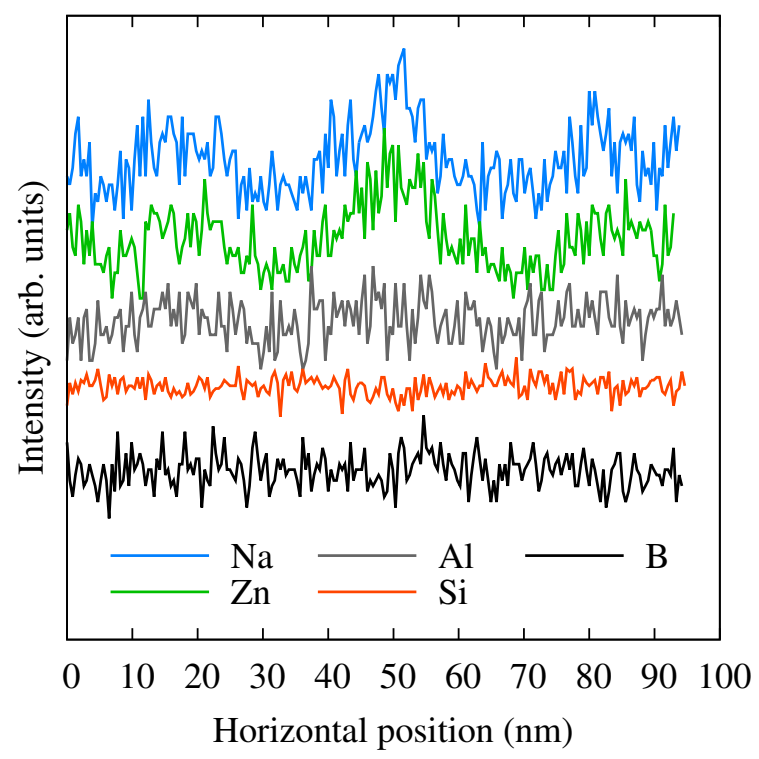

Figure 3.5: Concentration profiles extracted from Figures 3.4 on a $20 \mathrm{~nm}$ height band vertically centered 
irradiation with the best possible homogeneous dose in the samples and at a temperature well below the glass transition temperature has been developed.

\subsubsection{The T4 irradiation tube}

The High Flux Reactor of the ILL operates two main irradiation tubes, namely T4 and V4. The latter goes by the fuel assembly (around $75 \mathrm{~cm}$ from the center) and provides a mean neutron flux yielding $10^{15} \mathrm{n} / \mathrm{cm}^{2} / \mathrm{s}$. Because of the significant heat generated through the ${ }^{10} \mathrm{~B}(\mathrm{n}, \alpha)$ capture reactions $(\mathrm{Q} \simeq 2.5 \mathrm{MeV})$ and the technical constraints imposed by this setup (no contact accepted between the samples and the reactor cooling heavy water), irradiation at low temperature was not feasible at V4.

In contrast, the $\mathrm{T} 4$ device is a $50 \times 50 \mathrm{~mm}^{2}$ tube located in the light water reflecting vessel at a radial distance of $130 \mathrm{~cm}$ from the core center. The flux is nearly $100 \%$ thermal and the non perturbed values are about $2 \times 10^{13} \mathrm{n} / \mathrm{cm}^{2} / \mathrm{s}$, see Fig 3.6 and 3.8. Since such a setup is not considered to be "in-pile", its use is allowed under lesser stringent regulations although two thermal and radiological criteria have to be validated, which can be summarized as:

- The shuttle temperature has to be maintained below $100{ }^{\circ} \mathrm{C}$ all along the experiment, and

- $48 \mathrm{~h}$ after the end of irradiation, the dose rate at $250 \mathrm{~cm}$ from the shuttle loaded with samples has to be below $1 \mathrm{mGy} / \mathrm{h}$.

Because of the large heat production resulting from the ${ }^{10} \mathrm{~B}(\mathrm{n}, \alpha)$ reactions, a new irradiation shuttle had to be designed since the use of one already existing would have resulted in samples reaching a temperature above $700{ }^{\circ} \mathrm{C}$. Such temperature would have lead to unwanted annealing phenomena, and may have ended in damage of the aluminium shuttle itself. To remove the heat produced in the glass samples, a thin sample holder was built with two plates wrapping the $1.1 \mathrm{~mm}$ thick tiles with a square dimension of $10 \mathrm{~mm}$. The pristine glass provided by the neutron guide manufacturer could not be polished to a lower thickness. However, the mean flux in the sample was estimated to be $74 \%$ of the nominal one, which is acceptable for our experiments.

\subsubsection{Design, thermal, and radiological validation}

CAD drawings of the manufactured shuttle are displayed in Fig. 3.7 a-c. This new apparatus has been made in AG3net (a variant of the aluminium alloy AL5754, with a reduced content of the neutron-activating impurities $\mathrm{Cu}, \mathrm{Zn}$, etc.) and passivated to obey the nuclear safety rules of the ILL. As can be seen from the drawings, it is made of three elements assembled together by multiple titanium screws (Grade 5 - TA6V). The task of the blue and green plates is to trap and separates the samples while the dark grey element is a load made to ensure that the shuttle drops down to the bottom of the irradiation tube. The two plates have been vertically rabbeted to enhance the natural water flow. In order to let the water fill the voids around the samples and increase the heat conduction, a $0.2 \mathrm{~mm}$ slit was left between the two shuttle plates once assembled. Also, the whole shuttle was sized so that the middle of the sample column was located 


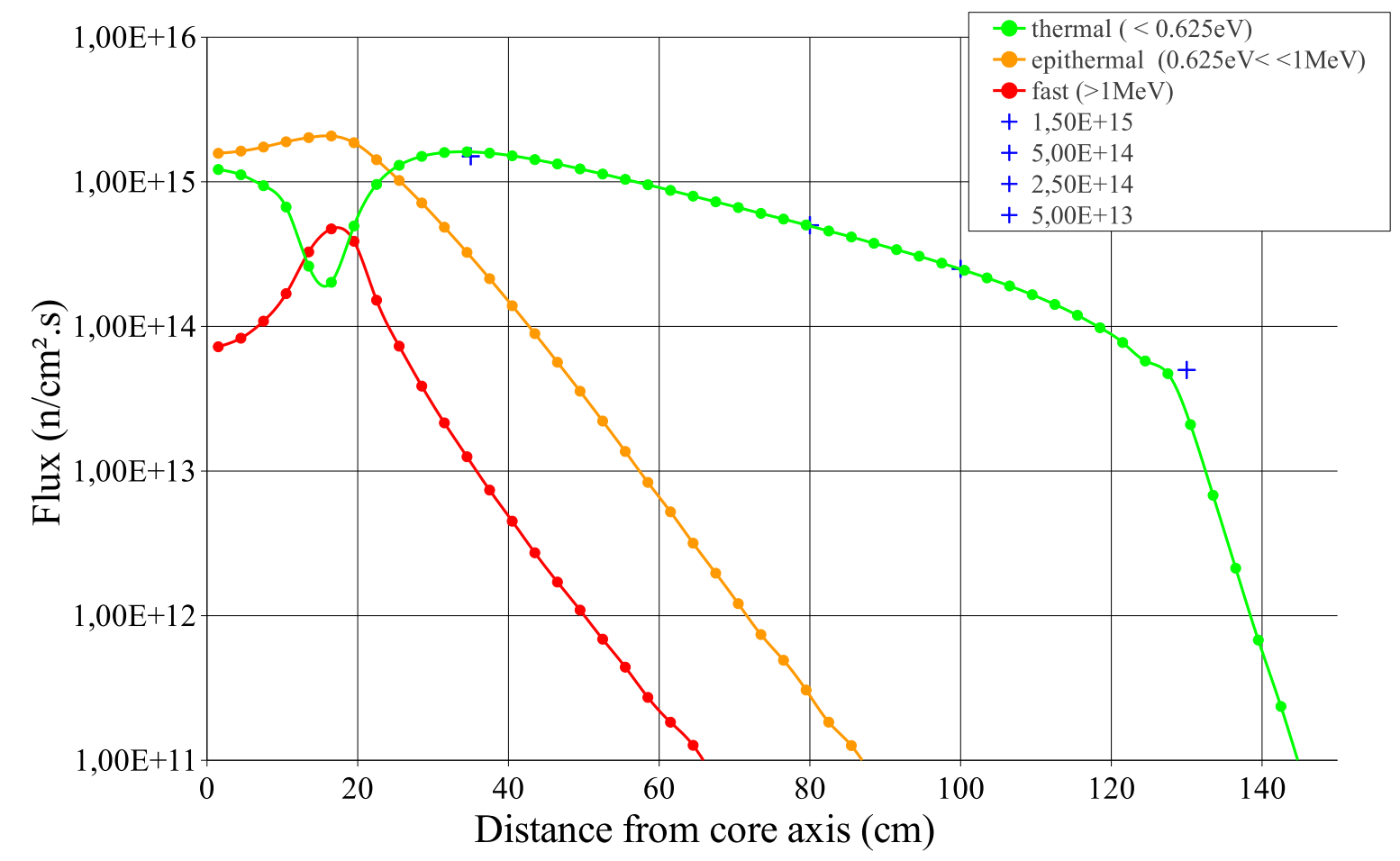

Figure 3.6: MCNP simulated flux in ILL's reactor as a function of the distance to the central axis; blue crosses are measured values (courtesy of ILL's Projects and Calculations Bureau).

in Core Median Plan (CMP) to have the most homogeneous flux for the whole sample batch.

Several thermal-hydraulic computations have been carried out in order to validate the thermal behaviour of this setup. The input heat sources were taken from MCNP and analytic calculation results. The heating in $\mathrm{T} 4$ due to nuclear reactions and gamma radiation had already been calculated for a sphere consisting in one gram aluminium (see the green curve on Fig 3.8). A value of $0.17 \mathrm{~W} / \mathrm{g}$ for the maximum power was assumed for the whole shuttle, even though its extremities are exposed to lower radiation levels. For the glass samples, in addition, the $(\mathrm{n}, \alpha)$ reactions had to be taken into account since they generate an important amount of energy which is trapped within the material, as most of it is shared by the ions. The blue curve of Fig 3.8 displays such a heat as calculated for N-ZK7,

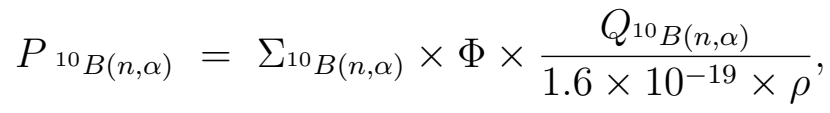

where $\Sigma_{10} B(n, \alpha)$ is the thermal macroscopic cross section of the ${ }^{10} B(\mathrm{n}, \alpha)$ reaction. For the computation, a figure for the nuclear heating ${ }^{1}$ of $12 \mathrm{~W} / \mathrm{g}$ was taken for all the samples. Let us mention that such a value is to be taken as a realistic upper bound, since it does not take into account the sample self-protection and/or the flux reduction due to the presence of other absorbing elements.

\footnotetext{
${ }^{1}$ By nuclear heating we refer to the heat deposited within the sample as a result of nuclear reactions and $\gamma$ rays.
} 


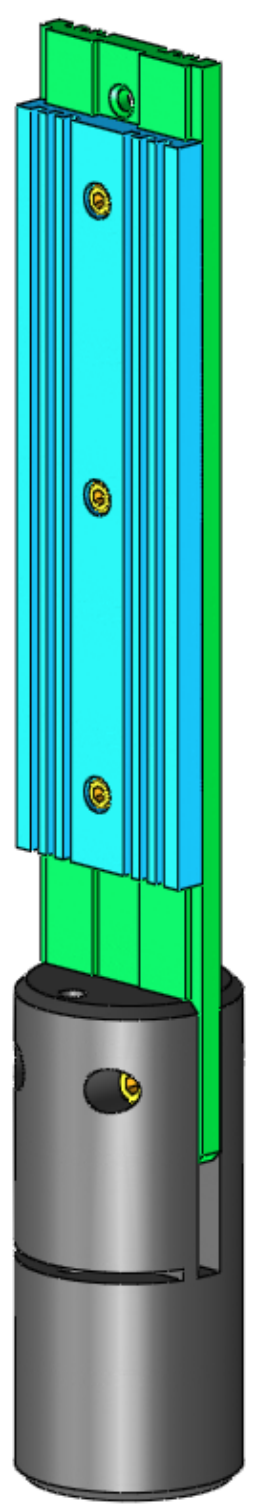

(a) Assembled view
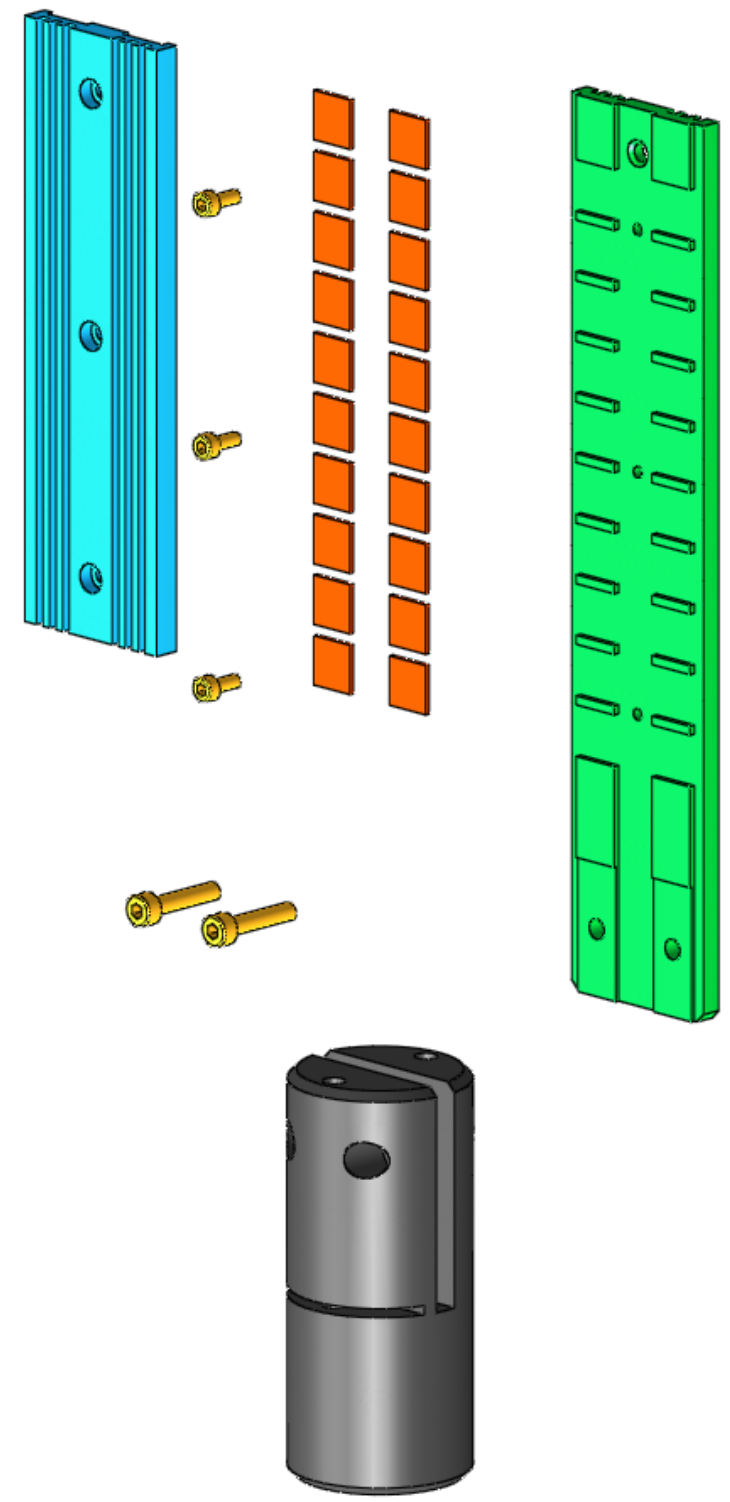

(b) Split view

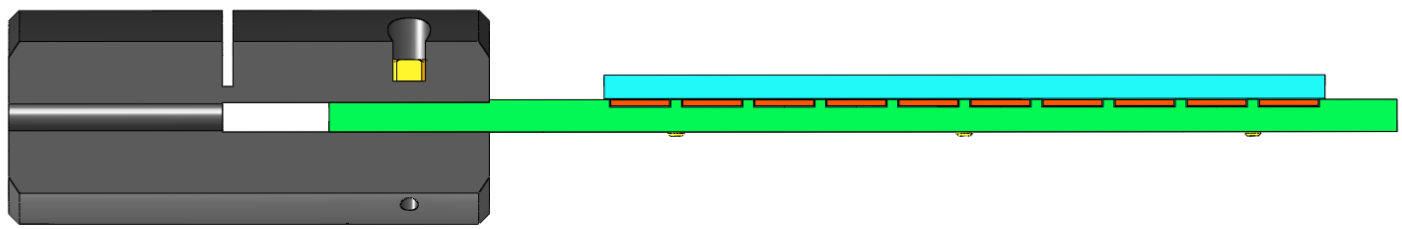

(c) Cut view along a sample column

Figure 3.7: T4 irradiation shuttle developed specifically to irradiate thin borosilicate tiles. CAD drawing, colors added to ease to comprehension. 


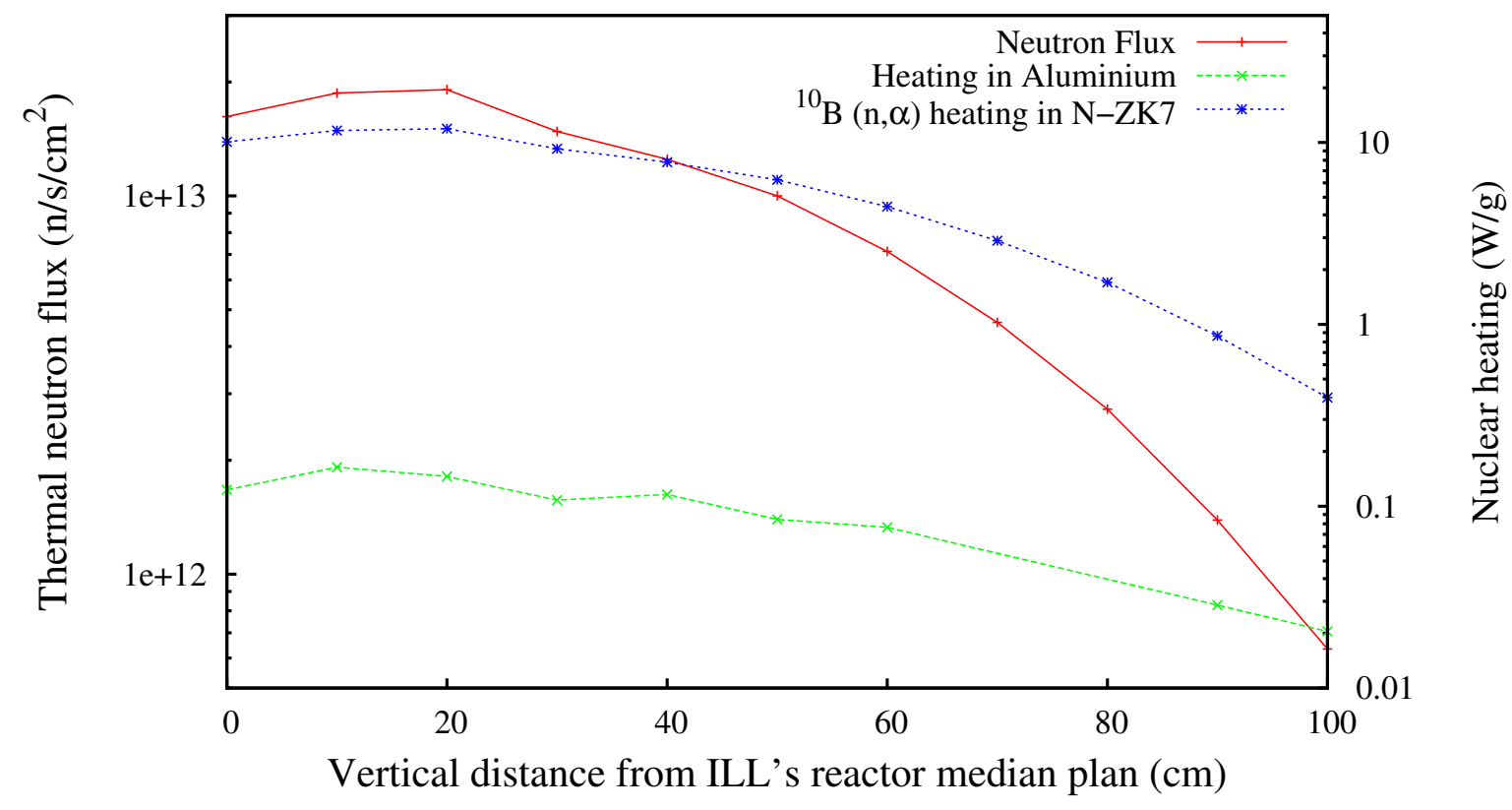

Figure 3.8: Flux and nuclear heating in T4 irradiation tube.

The results from the computational fluid dynamics can be gauged by inspection of Fig 3.9, which displays the maximum temperatures reached by the shuttle loaded with 20 generic borosilicate samples. It can be seen that the maximum temperature is around $63{ }^{\circ} \mathrm{C}$. Such temperature is acceptably low, since the glass transition temperature of all four glasses is around $550{ }^{\circ} \mathrm{C}$. Another advantage of the irradiation method employed here concerns the fact the thermal conditions under which the samples are subjected to are comparable to those of a real guide, that is, the glass is close to room temperature. It is also worth pointing out that guide heating due to neutron and $\gamma$ radiations only occur within the first five meters.

The radiological validation was done by combining results from two software packages, namely CASTOR and MicroShield. The former is an in-house developed tool that calculates the resulting activity from a given composition and irradiation characteristics. It details every stable and unstable nuclei and outputs their activity in Becquerel units. The second software was developed by EDF (Électricité De France) and computes the received dose as a function of the distance from the $\gamma$ spectrum . It can handle various source geometries and shielding types. To ease the calculations, CASTOR can generate files with MicroShield encoding. The CASTOR calculation characteristics are the following:

- a flux of $2 \times 10^{13} \mathrm{n} / \mathrm{cm}^{2} / \mathrm{s}$, which is pessimistic as the real perturbed flux will be described below,

- an irradiation time of 4 days,

- 4 samples for each glass type, and

- AG3net shuttle and titanium screws as reported in Tables 3.4 and 3.5 , respectively. 


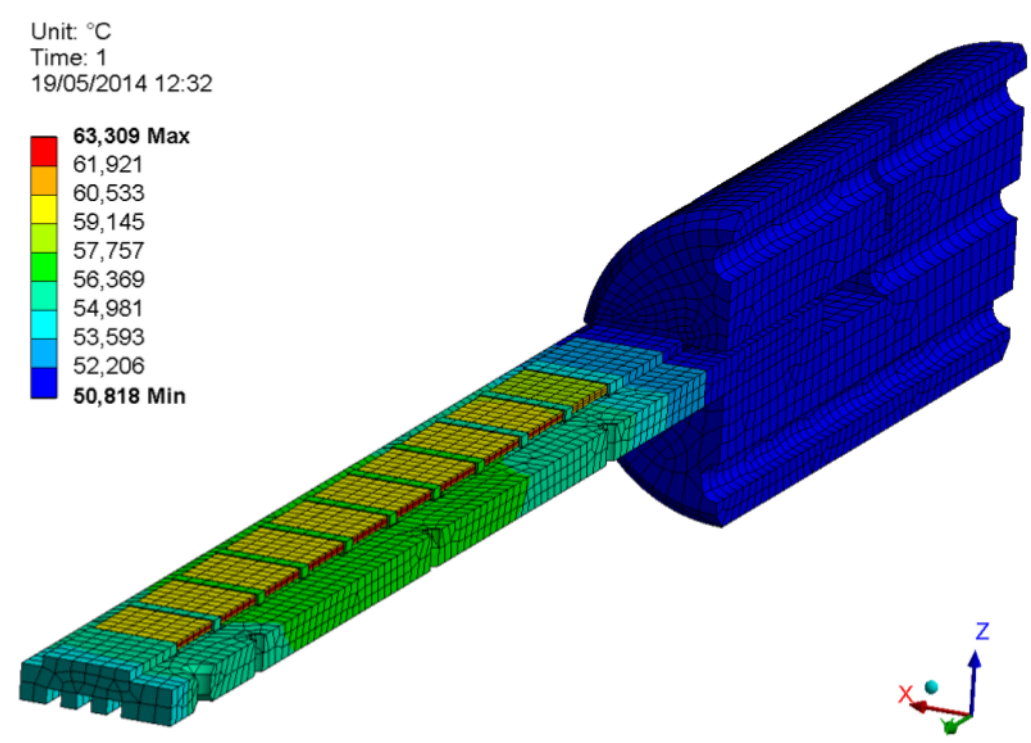

(a) Large view

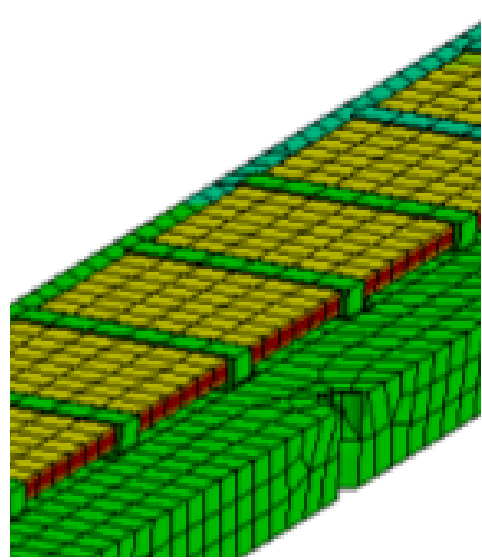

(b) Zoom

Figure 3.9: Computer fluid dynamics calculation showing the maximum temperature reached by the borosilicate glass samples when irradiated in T4. The blue plate from Fig 3.7 has not been shown, but was part of the simulation. Computations done by Francisco Garcia Sotto (ILL).

\begin{tabular}{cccccccccccccc}
\hline Atom & $\mathrm{Al}$ & $\mathrm{Si}$ & $\mathrm{Fe}$ & $\mathrm{Cu}$ & $\mathrm{Mn}$ & $\mathrm{Mg}$ & $\mathrm{Cr}$ & $\mathrm{Zn}$ & $\mathrm{B}$ & $\mathrm{Li}$ & $\mathrm{Cd}$ & $\mathrm{Pb}$ & $\mathrm{Sb}$ \\
Mass (g) & 381 & 1.2 & 1.6 & 0.032 & 2.8 & 12 & 1.2 & 0.12 & 0.004 & 0.004 & 0.004 & 0.04 & 0.04 \\
\hline
\end{tabular}

Table 3.4: Mass for the different elements in AG3net shuttle [1].

\begin{tabular}{c|ccccccc}
\hline Atom & $\mathrm{Ti}$ & $\mathrm{Al}$ & $\mathrm{V}$ & $\mathrm{Be}$ & $\mathrm{Cd}$ & $\mathrm{Co}$ & $\mathrm{Cr}$ \\
Mass (g) & 7.77 & 0.53 & 0.35 & $4.10^{-5}$ & $4.10^{-5}$ & $10^{-4}$ & $2.10^{-3}$ \\
\hline & $\mathrm{Cu}$ & $\mathrm{Fe}$ & $\mathrm{Hf}$ & $\mathrm{Mn}$ & $\mathrm{Mo}$ & $\mathrm{Ni}$ & $\mathrm{Pd}$ \\
& $10^{-4}$ & 0.014 & $10^{-4}$ & $2.10^{-4}$ & $10^{-4}$ & 0.002 & $10^{-4}$ \\
\hline
\end{tabular}

Table 3.5: Mass for the different elements in Grade-5 titanium screws 52. 


\begin{tabular}{cc|cc}
\hline \multicolumn{2}{c|}{48 h decay } & \multicolumn{2}{c}{30 decay } \\
Nucleus & Activity (Bq) & Nucleus & Activity (Bq) \\
\hline${ }^{51} \mathrm{Cr}$ & $1.7 \cdot 10^{10}$ & ${ }^{51} \mathrm{Cr}$ & $8.6 \cdot 10^{9}$ \\
${ }^{24} \mathrm{Na}$ & $7.4 \cdot 10^{9}$ & ${ }^{124} \mathrm{Sb}_{G}$ & $3.0 \cdot 10^{8}$ \\
${ }^{122} \mathrm{Sb}_{G}$ & $6.4 \cdot 10^{9}$ & ${ }^{65} \mathrm{Zn}$ & $1.5 \cdot 10^{8}$ \\
${ }^{64} \mathrm{Cu}$ & $1.4 \cdot 10^{9}$ & ${ }^{55} \mathrm{Fe}$ & $1.2 \cdot 10^{8}$ \\
${ }^{124} \mathrm{Sb}_{G}$ & $3.7 \cdot 10^{8}$ & ${ }^{59} \mathrm{Fe}$ & $4.7 \cdot 10^{7}$ \\
\hline
\end{tabular}

Table 3.6: Post irradiation activity of the new T4 shuttle loaded with 16 thin glass samples with 4 days of irradiation at $2 \times 10^{13} \mathrm{n} / \mathrm{cm}^{2} / \mathrm{s}$. G indicates that the decay reactions are from the ground state of the nucleus.

The main radionuclides are reported in Table 3.6 for the irradiation characteristics mentioned above. Two decay times have been computed, 48 hours and 30 days. One can see that after $48 \mathrm{~h}$ of decay, the activity should be dominated by the ${ }^{51} \mathrm{Cr}$ nuclei $\left(\mathrm{T}_{1 / 2}=27.7 \mathrm{~d}\right)$ produced in the shuttle itself, the second most active element being the ${ }^{24} \mathrm{Na}\left(\mathrm{T}_{1 / 2}=14.96 \mathrm{~h}\right)$ from the glass samples. In fact, it is this latter $\gamma$ emitter that controls the moment when one can access the neutron guides once the beam has been shut off. After 1 month of cooling, the shuttle activity should still be dominated by ${ }^{51} \mathrm{Cr}$, while the glasses are mostly active because of ${ }^{65} \mathrm{Zn}$ and ${ }^{124} \mathrm{Sb}$ with 244.3 days and 60.3 days half-life, respectively.

The dose rate computation done with MicroShield used the $\gamma$ emitters forecast by CASTOR after $48 \mathrm{~h}$ of decay. As the calculation has to be at $250 \mathrm{~cm}$ from the source, it latter was considered as a point geometry. The software yielded a value of $0.66 \mathrm{mGy} / \mathrm{h}$, which validates the radiological criterion for the use of T4. A similar calculation after 30 days of decay gave a value of $36 \mu \mathrm{Gy} / \mathrm{h}$. This latter figure shows that the recovery of the samples that will have to be made by hand will impose precautions to reduce the dose received by the operators.

\subsubsection{In-situ flux measurement}

The term "non-perturbed" flux at a given position means that no foreign absorbing or scattering object is located at that position. In the case of T4, the "rest position" of the system is light water filling an aluminium square tube. In the case of a small aluminium object inserted in that system, the neutron flux would not be largely modified, since both scattering and absorption cross-sections for aluminium are reasonably low. However, in the case of borosilicate glasses, the strong absorption power on ${ }^{10} \mathrm{~B}$ nuclei would noticeably reduce the flux. To monitor that effect, thin zirconium foils have been added to the shuttles. It must be mentioned that gold foils are usually used to monitor the flux in neutron guides, but zirconium has been preferred in our case because of its smaller absorption cross section and longer half life.

Among the different zirconium isotopes, it is the thermal neutron activation of ${ }^{94} \mathrm{Zr}$ that 
is of interest to us. The activation and decay chain is the following:

$$
\begin{aligned}
\quad 94 \mathrm{Zr}+n \rightarrow{ }^{95} \mathrm{Zr} & \sigma_{a}=0.05 b \\
{ }^{95} \mathrm{Zr} \rightarrow{ }^{95} \mathrm{Nb}+\beta^{-}+\gamma & T_{1 / 2}=64 d \\
{ }^{95} \mathrm{Zr} \rightarrow{ }^{95} \mathrm{Mo}+\beta^{-}+\gamma & T_{1 / 2}=35 d
\end{aligned}
$$

The foil thickness used was $25 \mu \mathrm{m}$. It induces an auto protection below $10^{-3}$, which is low enough to consider that the foil is homogeneously irradiated. To have enough statistics and still avoid saturation of the $\gamma$ detector, an activity around $10 \mathrm{kBq}$ per foil was aimed. If we consider an irradiation time $T_{i}=4 d$, a decay time $T_{d}=30 d$, and an irradiation flux $\phi_{0}=2 \times 10^{13} \mathrm{n} / \mathrm{cm}^{2} / \mathrm{s}$, the activity at the end of the decay period is,

$$
A(30 j)=N_{Z r-94} \cdot \phi_{0} \cdot \sigma_{a}\left(1-e^{-\lambda_{Z r-94} \cdot T_{i}}\right) \cdot e^{-\lambda_{Z r-94} \cdot T_{d}}
$$

where $N_{Z r-94}$ and $\lambda_{Z r-94}$ are the ${ }^{94} \mathrm{Zr}$ atomic density and decay constant respectively. This equation gives $2 \times 2 \mathrm{~mm}^{2}$ foils an activity of $22 \mathrm{kBq}$. This dimension complies with the detector constraint and is handy to manipulate.

At least two flux monitors were added per shuttle, one per side, in order to have flux values on both sample sides. Each of them was wrapped within an aluminium foil with typical dimensions of $5 \times 5 \mathrm{~mm}^{2}$. This was done to avoid the zirconium foil from gliding from one sample spot to another, or even exiting the shuttle. The $\gamma$ counting was carried out using two high purity germanium detectors, namely the models Eurisys EGPC 30-185-R and 25-185-R. The samples were installed $75 \mathrm{~mm}$ above the cell, a distance at which the machine had been calibrated with a source of well known activity.

The different measurements yielded a mean perturbed flux per shuttle of $6.7 \times 10^{12} \pm$ $0.4 \times 10^{12} \mathrm{n} / \mathrm{s} / \mathrm{cm}^{2}$. For a few foils used in the same irradiation, the flux variation could differ from 6 to $15 \%$ at most. Such differences were attributed to the orientation of the shuttle with respect to the reactor core, that is whether the shuttle front face was collinear to the reactor radius or if there was some mismatch in its positioning. This parameter induces a perturbed flux more or less homogeneous on both sides of the samples and hence on the two foils.

\subsubsection{Some pictures of the experiments}

Figure 3.10a is a view from the reactor pool level of the T4 entrance. On that picture one can see a prototype shuttle inserted in T4 to validate the dimensions. At that moment the pool was empty. Figure $3.10 \mathrm{c}$ is a photo of the dismantling of the load from the plates. This was done to remove the most active part of the apparatus. This manipulation was done by reactor and nuclear health agents authorized to work under ionizing conditions higher than a thesis student. Finally, Fig $3.10 \mathrm{~b}$ shows the setup organized to recover the irradiated glass pieces from the shuttles. 


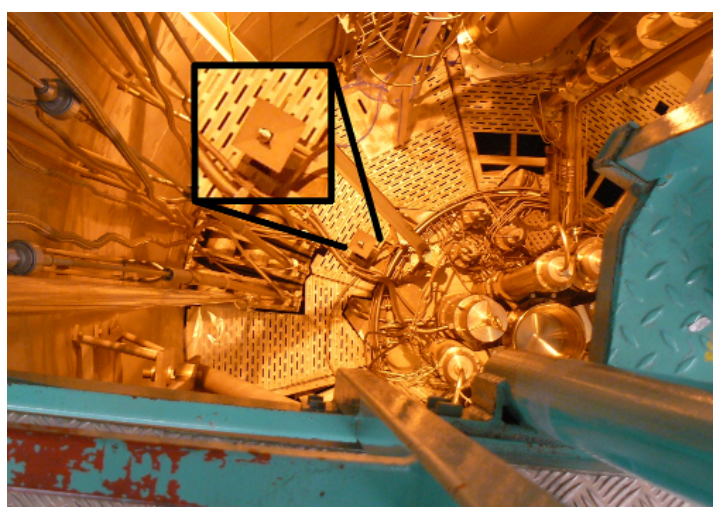

(a) Shuttle prototype test insertion in T4, view from reactor pool level.

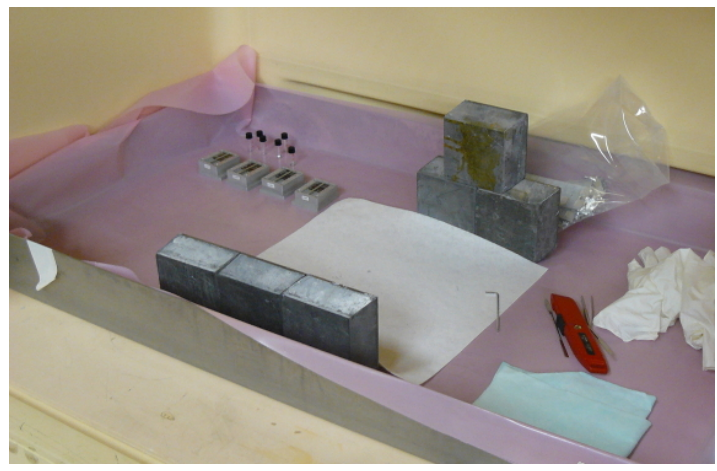

(b) Recovering irradiated sample. Storing boxes on top left hand side; remaining filled shuttles behind lead brick on the top right hand side.

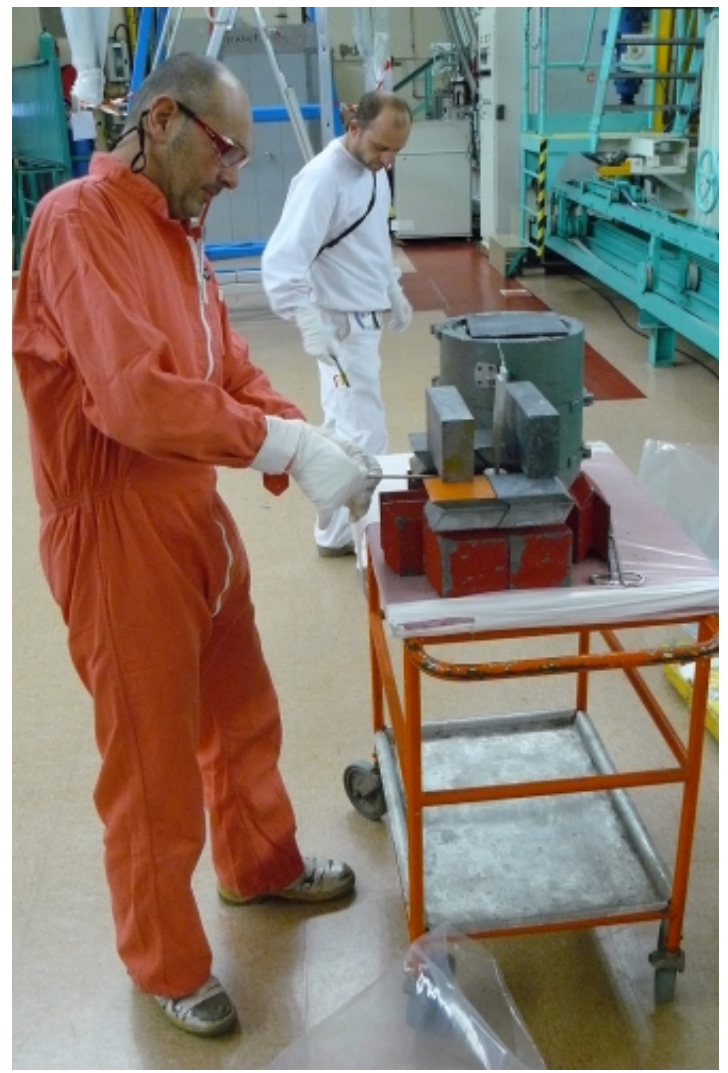

(c) Post irradiation dismantling of shuttle load element. E. Mannino doing doing the operation under P. Cochet supervision.

Figure 3.10: Thin sample irradiation in T4, photos of the experiment. 


\subsection{Gamma ray spectroscopy}

In order to check the elementary chemical analysis, and also to understand the nature of radiation activation processes within the glassy matrix, several measurements using gamma spectroscopy have been carried out. This was done by means of irradiating small amounts of glass substrates with thermal neutrons, thus enabling us to compare the parameters derived from the gamma spectra with those expected from the known chemical composition.

\subsubsection{Experimental Procedure}

The $\gamma$ spectra was recorded using $1.1 \mathrm{~mm}$ thick glass samples irradiated in the $\mathrm{T} 4$ facility for 7 hours. The spectrometers employed here are the same as for the zirconium foil. The measurements were done 70 days after the end of irradiation. For that reason, all of the short lived actinides could not be detected.

The CASTOR software was used to forecast the samples' radio-activity. Starting from the amounts of nuclei, neutron flux, and irradiation time, the software calculates all the other nuclei produced by considering the different neutron reactions $((n, \gamma),(n, p),(n, \alpha)$ etc.) and disintegration reactions $(\beta-, \beta+, \alpha)$. The computer output yields the different nuclei in the sample following irradiation and their activity as a function of the decay time. To take into account the self-shielding phenomenon occurring in the samples due to the high boron-10 content, the values for the material quantity entering the calculation were multiplied by a factor of 0.74 .

\subsubsection{Results}

The $\gamma$ ray measurements and the results from calculations are listed in Table 3.7. Only nuclei detected during the measurements are displayed. One can see that there is a fairly good agreement between the predicted and detected $\gamma$ intensities, especially for the main $\gamma$ emitters. In the case of N-ZK7 and N-BK7, the small variety of detected radioisotopes comes from the screening of a large part of the energy spectrum caused by the relatively high intensities of ${ }^{65} \mathrm{Zn}$ and ${ }^{124} \mathrm{Sb}$ and their associated Compton effect in the germanium cells. This phenomenon is known as the Compton front.

The most active glass after irradiation is N-ZK7, due to its large zinc and antimony content and the long half-lives of their activation products (244.3 and 60.3 days respectively). Then come N-BK7 and S-BSL7, again having antimony and zinc as the main activation products. Finally, the activity of Borofloat is mainly influenced by the trace elements.

The results from these set of experiments squares with the results of the chemical analysis. Moreover, even if the short-lived actinides had already decayed, it highlights that the use of some of the candidate materials for glass substrates, such as N-ZK7, should be planned with care due to the relatively high amount of long-lived radioisotopes generated. 


\begin{tabular}{c|cc|cc|cc|cc}
\hline & \multicolumn{2}{|c|}{ Borofloat } & \multicolumn{2}{c|}{ N-ZK7 } & \multicolumn{2}{c|}{ N-BK7 } & \multicolumn{2}{c}{ S-BSL7 } \\
\hline & Measured & Calculated & Measured & Calculated & Measured & Calculated & Measured & Calculated \\
\hline${ }^{46} \mathrm{Sc}$ & $6.2 \cdot 10^{2}$ & - & - & - & - & - & $3.3 \cdot 10^{2}$ & - \\
${ }^{51} \mathrm{Cr}$ & $3.6 \cdot 10^{1}$ & - & - & - & - & - & - & - \\
${ }^{59} \mathrm{Fe}$ & $6.4 \cdot 10^{1}$ & $1.04 \cdot 10^{2}$ & - & & & & & \\
${ }^{60} \mathrm{Co}$ & $1.9 \cdot 10^{1}$ & - & - & - & - & - & 8.5 & - \\
${ }^{65} \mathrm{Zn}$ & $1.1 \cdot 10^{2}$ & $1.73 \cdot 10^{3}$ & $1.1 \cdot 10^{6}$ & $3.55 \cdot 10^{6}$ & - & - & $2.0 \cdot 10^{5}$ & $3.22 \cdot 10^{5}$ \\
${ }^{95} \mathrm{Zr}$ & $4.2 \cdot 10^{2}$ & $7.36 \cdot 10^{2}$ & - & - & - & - & $2.1 \cdot 10^{2}$ & $5.79 \cdot 10^{2}$ \\
${ }^{124} \mathrm{Sb}$ & $3.7 \cdot 10^{1}$ & $4.16 \cdot 10^{2}$ & $2.9 \cdot 10^{5}$ & $1.05 \cdot 10^{6}$ & $3.0 \cdot 10^{5}$ & $4.1410^{5}$ & $4.410^{4}$ & $6.6810^{4}$ \\
${ }^{131} \mathrm{Ba}$ & - & - & - & - & $1.1 \cdot 10^{3}$ & $9.18 \cdot 10^{2}$ & $1.110^{3}$ & $2.2210^{3}$ \\
${ }^{133} \mathrm{Ba}$ & - & - & - & - & $2.8 \cdot 10^{2}$ & $1.3 \cdot 10^{2}$ & $3.2 \cdot 10^{2}$ & $3.15 \cdot 10^{2}$ \\
${ }^{134} \mathrm{Cs}$ & $2.7 \cdot 10^{2}$ & - & - & - & - & - & - & - \\
${ }^{141} \mathrm{Ce}$ & $8.0 \cdot 10^{1}$ & $2.05 \cdot 10^{2}$ & - & - & - & - & - & - \\
${ }^{152} \mathrm{Eu}$ & $9.1 \cdot 10^{1}$ & - & - & - & - & - & - & - \\
${ }^{160} \mathrm{~Tb}$ & $2.9 \cdot 10^{1}$ & - & - & - & - & - & - & - \\
${ }^{169} \mathrm{Yb}$ & $1.6 \cdot 10^{2}$ & - & - & - & - & - & - & - \\
${ }^{181} \mathrm{Hf}$ & $2.7 \cdot 10^{3}$ & $3.84 \cdot 10^{2}$ & & - & & & $1.7 \cdot 10^{3}$ & $3.84 \cdot 10^{2}$ \\
${ }^{233} \mathrm{~Pa}$ & $6.3 \cdot 10^{1}$ & - & - & - & - & - & - & - \\
\hline
\end{tabular}

Table 3.7: Gamma activity, measured and calculated, per gram of material for the four different glasses after 70 days of decay. Only the experimentally detected nuclei are shown. Seven hours of irradiation under $2 \times 10^{13} \mathrm{n} / \mathrm{cm}^{2} / \mathrm{s}$. Values in $\mathrm{Bq} / \mathrm{g}$.

\subsection{Bulk density evolution under thermal neutron flux}

\subsubsection{Experimental procedure}

Knowledge derived from published reports attributes the mechanical failure of the mirrors to a modification of the glass density in and around the irradiated zone, whereas the rest of the material remains unaffected $[78,79]$. Motivated by such lines of reasoning, we have measured the evolution of the density of the different glasses as a function of the received neutron fluence. The samples employed for such an endeavour had dimensions of $10 \times 10 \mathrm{~mm}^{2}$ and thickness of $1.1 \mathrm{~mm}$, and were mounted in the irradiation shuttle described in section 3.4 .

An important issue to consider concerns the estimation of the average flux received across the sample, accounting for the self-attenuation effects resulting from the ${ }^{10} \mathrm{~B}$ high absorption cross section. As a first order approximation, one can calculate the selfattenuation $v$ for an infinite plate with thickness $w$, which leads to an expression for the attenuated flux coming from one side of the plate at a distance $z$ from the surface as,

$$
\phi=\int \phi_{0} \frac{\mathrm{d} \Omega}{4 \pi} \mathrm{e}^{-\frac{z \Sigma_{a}}{\cos (\theta)}}
$$

where $\Omega$ is the solid angle of the outer neutron source as seen at depth $z$. This can be 
rewritten in spherical coordinates as

$$
\begin{aligned}
& \mathrm{d} \Omega=\frac{\mathrm{d} \mathbf{S} \cdot \mathbf{u}}{z^{2}} \\
& \mathrm{~d} \Omega=2 \pi z^{2} \sin (\theta) \mathrm{d} \theta
\end{aligned}
$$

The resulting reaction rate for a plate irradiated from both sides is then,

$$
\tau(z, w)=\frac{\Sigma_{a} \phi_{0}}{2} \int_{0}^{\pi / 2}\left(\sin (\theta) \mathrm{e}^{-\frac{z \Sigma_{a}}{\cos (\theta)}}+\sin (\theta) \mathrm{e}^{-\frac{(w-z) \Sigma_{a}}{\cos (\theta)}}\right) \mathrm{d} \theta
$$

To solve this equation, one has to make a variable substitution

$$
\begin{aligned}
& t=\cos (\theta) \\
& \mathrm{d} t=-\sin (\theta) \mathrm{d} \theta
\end{aligned}
$$

then,

$$
\tau(z, w)=\frac{\Sigma_{a} \phi_{0}}{2} \int_{0}^{1}\left(e^{-\frac{z \Sigma_{a}}{t}}+e^{-\frac{(w-z) \Sigma_{a}}{t}}\right) \mathrm{d} t
$$

In order to go further, one has to recall the tabulated $\mathrm{E}_{n}(\mathrm{x})$ integrals 19 ,

$$
\begin{gathered}
E_{n}(x)=\int_{1}^{\mathrm{inf}} \frac{\mathrm{e}^{-u x}}{u^{n}} \mathrm{~d} u \\
E_{n}(x)=\int_{0}^{1} t^{n-2} \mathrm{e}^{-\frac{x}{t}} \mathrm{~d} t
\end{gathered}
$$

which gives

$$
\tau(z, w)=\Sigma_{a} \phi_{0} E_{2}\left(\Sigma_{a} z\right)
$$

Thanks to this formula, one can calculate the flux in the sample at any depth. Considering our experiment, it is more interesting to evaluate the mean reaction rate for the whole sample:

$$
\begin{aligned}
\bar{\tau}(w) & =\int_{z=0}^{w} \tau(z, w) \mathrm{d} z \\
& =\frac{\Sigma_{a} \phi_{0}}{2} \int_{t=0}^{1} \int_{z=0}^{w}\left(\mathrm{e}^{-\frac{z \Sigma_{a}}{t}}+\mathrm{e}^{-\frac{(w-z) \Sigma_{a}}{t}}\right) \mathrm{d} z \mathrm{~d} t \\
& =\phi_{0} \cdot \frac{1 / 2-E_{3}\left(\Sigma_{a} w\right)}{w} \\
\bar{\tau}(w) & =\phi_{0} \Sigma_{a} \cdot \frac{1 / 2-E_{3}\left(\Sigma_{a} w\right)}{\Sigma_{a} w},
\end{aligned}
$$

from where it is possible to extract the self-attenuation factor $v$,

$$
v=\frac{1 / 2-E_{3}\left(\Sigma_{a} w\right)}{\Sigma_{a} w}
$$




\begin{tabular}{c|ccccc}
\hline Irradiation fluence & $\mathrm{F} 1$ & $\mathrm{~F} 2$ & $\mathrm{~F} 3$ & $\mathrm{~F} 4$ & $\mathrm{~F} 5$ \\
\hline Fluence $\left(\mathrm{n} \mathrm{cm}^{-2}\right)$ & $1.3 \cdot 10^{17}$ & $2.9 \cdot 10^{17}$ & $3.9 \cdot 10^{17}$ & $7.6 \cdot 10^{17}$ & $2.2 \cdot 10^{18}$ \\
$(\mathrm{n}, \alpha)$ reactions $\left(\mathrm{cm}^{-3}\right)$ & $5.1 \cdot 10^{17}$ & $1.1 \cdot 10^{18}$ & $1.5 \cdot 10^{18}$ & $3.0 \cdot 10^{18}$ & $8.7 \cdot 10^{18}$ \\
\hline $\left.\mathrm{E}_{\text {ioniz. }}(\mathrm{keV} \mathrm{cm})^{-3}\right)$ & $1.2 \cdot 10^{21}$ & $2.6 \cdot 10^{21}$ & $3.5 \cdot 10^{21}$ & $6.9 \cdot 10^{21}$ & $2.0 \cdot 10^{22}$ \\
$\left.\mathrm{E}_{\text {nucl. }}(\mathrm{keV} \mathrm{cm})^{-3}\right)$ & $1.7 \cdot 10^{19}$ & $3.8 \cdot 10^{19}$ & $5.1 \cdot 10^{19}$ & $1.0 \cdot 10^{20}$ & $2.9 \cdot 10^{20}$ \\
$\mathrm{dpa}$ & 0.0041 & 0.0091 & 0.012 & 0.024 & 0.070 \\
\hline
\end{tabular}

Table 3.8: Fluence resulting from homogeneous irradiation and the corresponding deposited energy and dpa calculated with SRIM [121].

which yields an average flux for the whole sample of $74 \%$ from that received at the surface.

To follow the density evolution of the glasses with received neutron dose, we have employed the hydrostatic weighting method. A density determination kit has been installed on a Mettler-Toledo AE240 weighting scale. Measurements on the non irradiated samples have shown that this setup could reach a standard deviation of about $0.05 \%$, which is low enough considering the expected variations which are one order of magnitude larger [8]. For each glass and neutron dose, four glass tiles were installed in the shuttle, which made a total of 16 samples per shuttle. For the measurements on irradiated and hence active samples, the procedure was considerably modified. The samples were grouped and measured per glass type. It started with the least active sample, Borofloat, and finished with the most active one, N-ZK7. When one specimen was manipulated and weighed several times, the others were screened behind $5 \mathrm{~cm}$ thick lead bricks. In the specific case of the most active N-ZK7 samples, which showed a contact dose around $4 \mathrm{mSv} / \mathrm{h}$, each measured glass piece was also screened behind lead when the scale was stabilizing between each measurement. The details on the different fluences are reported in Table 3.8. the values take into account the perturbed flux measured by Zr foils and the self-attenuation factor.

\subsubsection{Results}

The data showing the density variation of the different glasses with received dose are displayed in Fig 3.11. The error bars shown there display the standard deviation calculated from the measured values. Also, for each sample, at least four weighings have been done in order to ensure the reproducibility. One can see from the graph that Borofloat is the sample most affected by radiation. In fact, its density increases up to $2 \%$ for a fluence of about $0.5 \times 10^{18} \mathrm{n} / \mathrm{cm}^{2}$, and then slightly decreases at higher fluence values. The N-ZK7 follows the same general trend, but shows less marked features. Its density also increases, but only up to $1 \%$. In the case of N-BK7 and S-BSL7, the opposite behaviour is observed, that is their structure swells under neutron fluence. In fact, the data shows that up to a fluence of $0.7 \times 10^{18} \mathrm{n} / \mathrm{cm}^{2}$, their density changes much less than the two other glasses. Above this value, their structure continues swelling and in fact, the absolute density change surpasses that of N-ZK7.

Regarding the density variations upon the received dose, it is pertinent to recall 


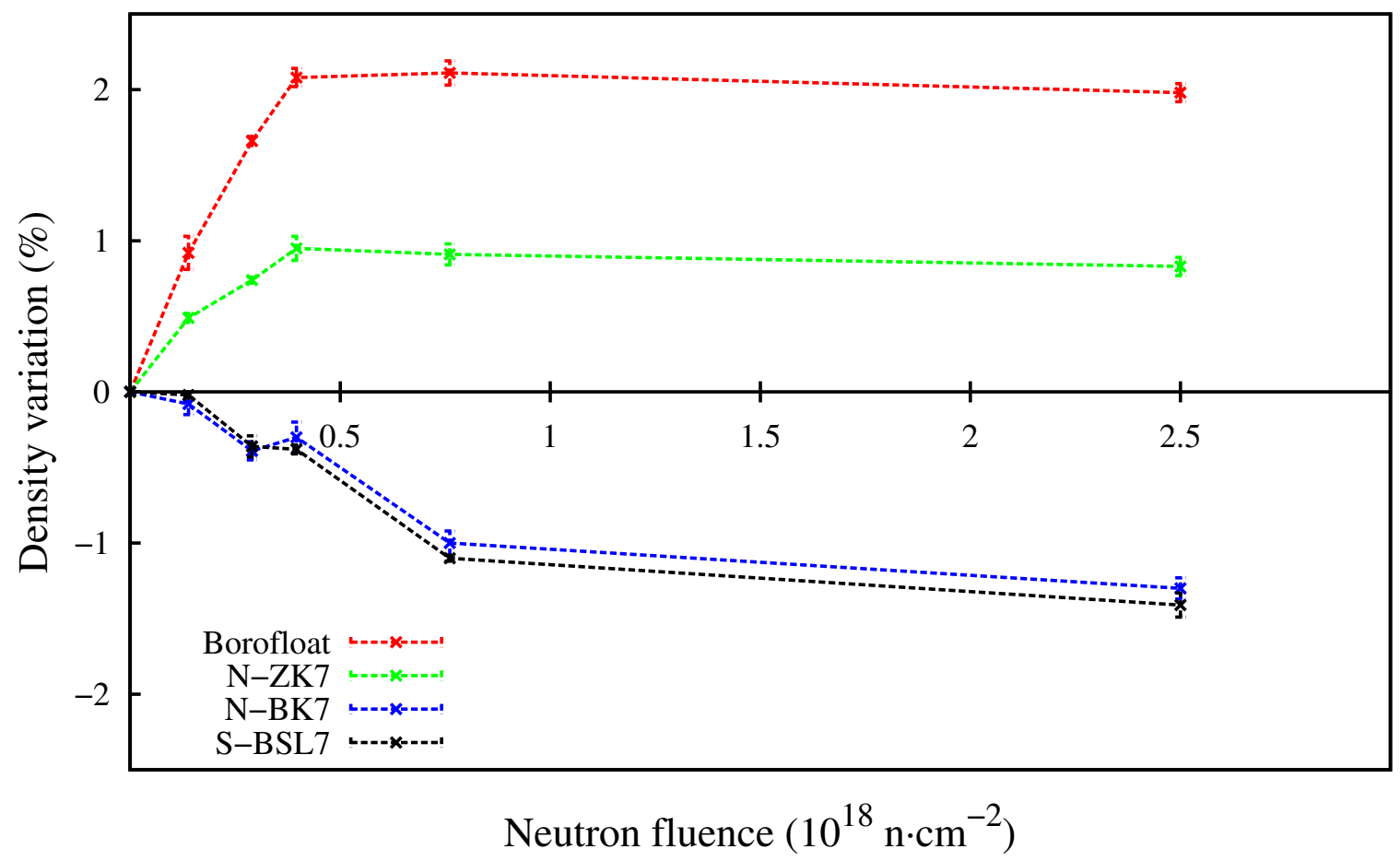

Figure 3.11: Density evolution of glasses as a function of received fluence. Homogeneous neutron irradiation at a temperature below $65{ }^{\circ} \mathrm{C}$. Lines drawn between the points to ease the reading.

the pioneering studies of Simon [103], Weissman [108] and Bale [15], carried out long ago for vitreous silica under neutron radiation. Such results suggested the presence of rod-like defect clusters generated by thermal spikes within the glass matrix. The irradiation induced shrinking of amorphous $\mathrm{SiO}_{2}$ has been correlated more recently with an increase of the small silicon ring concentration and the associated decrease of the Si-O-Si bond angle $[32,35]$. In contrast, further studies using a variety of radiation sources $6,13,17,50,93,118]$ show less marked effects on vitreous silica when irradiated with electrons and photons than those found for vitreous borosilicate. For this kind of glass, the macroscopic evolution under irradiation has also been correlated with structural changes 83]. Raman spectroscopy has shown that the Si-O-Si angle decreases with increasing dose in the case of gold ion irradiated samples [31], which is a typical feature of the silica network. In addition, the observation of swelling of some alkali-borosilicate systems has been linked to the evolution of the boron coordination number from four-fold to threefold 75. Therefore, by looking at their chemical composition, it appears that Borofloat and N-ZK7, with their low alkali content, have a behaviour that could be related to that of pure silica, which results in significant shrinking. In contrast, N-BK7 and S-BSL7, with a higher network modifier concentration, show a macroscopic behaviour closer to one generally reported for borosilicate: swelling [81.

The radiation hardness limits resulting from the H112/H21 experiments may be related to the observed density variation curves, see Table 2.4 on $\mathrm{p} .39$. We see that the explored fluence level in $\mathrm{H} 112 / \mathrm{H} 21$ are below $0.5 \times 10^{18} \mathrm{n} . \mathrm{cm}^{-2}$, which correspond to the high "reactivity zone" of Borofloat and N-ZK7 and the very slow swelling regime of 
N-BK7 and S-BSL7. The glass shrinking under the mirror for Borofloat and N-ZK7 will generate tensile stresses that lead to surface cracking. For the two other glasses, such stresses are located deeper within the mirror plate. One can estimate that their fracture could occur, but at much higher fluence and would initiate under the surface.

\section{Low fluence regime}

Following the results detailed in the previous paragraph, we decided to explore the macroscopic behaviour of the glasses at fluence below $4 \times 10^{17} \mathrm{n} / \mathrm{cm}^{2}$. The interest of such an exercise is to confirm whether the macroscopic behaviour of the glasses under irradiation is linear, as suggested by Fig. 3.11. To do so, irradiation ageing experiments were done in the similar conditions as those described previously. Shuttles were loaded with 4 samples per glass type together with zirconium foils for the flux monitoring. The irradiation time in T4 lasted from 5.5 minutes to 7 hours, corresponding to perturbed fluences from $0.019 \times 10^{17}$ to $1.2 \times 10^{17} \mathrm{n} / \mathrm{cm}^{2}$.

The density evolution of the glasses is reported in Fig. 3.12a. For this experiment, the sample density was only measured after irradiation. The reference value was taken from the high fluence experiment. As expected, the glasses can be grouped into two types. The N-BK7 and S-BSL7 materials show no significant density variation as a function of the received neutron dose, as the swelling of the glass structure stays below $0.1 \%$. In contrast, Borofloat and N-ZK7 density is strongly dependent upon the received dose. Even though the first values appear to be quite scattered, the dominant trend is roughly linear. Figure $3.12 \mathrm{~b}$ shows the density measurements of the two experiments up to a fluence around $4 \times 10^{17} \mathrm{n} / \mathrm{cm}^{2}$ for Borofloat and N-ZK7. Data were fitted using linear functions and the resulting line is also reported in the plot. This gives density variation coefficients for Borofloat and N-ZK7 of $5.8 \times 10^{-20}$ and $2.5 \times 10^{-20}(\Delta \rho / \rho) /\left(\mathrm{n} / \mathrm{cm}^{2}\right)$, respectively. Such results could be used to simulate the generation of stress inside the glass plates under neutron flux.

As can be inferred from the smallest fluence values explored, the present method is not well suited to explore neutron doses below $5 \times 10^{16} \mathrm{n} / \mathrm{cm}^{2}$. In order to increase its accuracy, one could employ thinner samples leading to a more homogeneous flux distribution. Also, we can think of using a less intense irradiation tube. Indeed, the insertion and extraction takes time, which brings a minimum irradiation limit of few minutes. However, these results are consistent with those obtained from Allred et al. [8] in an ageing experiment where they followed the density evolution of Pyrex® as a function of the thermal neutron flux. In their work, they found a slope of $8.14 \times 10^{-20}(\Delta \rho$ $/ \rho) /\left(\mathrm{n} / \mathrm{cm}^{2}\right)$. In fact, using only the 5 lowest fluence points, the best fitting slope appears to be $9.1 \times 10^{-20}$. The results presented here thus lend additional support to the picture that portrays the breakdown of Borofloat and N-ZK7 plates resulting from a compaction of the glass structure. Conversely, the observed stability of N-BK7 and S-BSL7 during the H112 irradiation is due to the very low correlation of their density to the received dose. Those two last glasses might show some brittle rupture phenomena if they are brought to very high neutron fluences. 


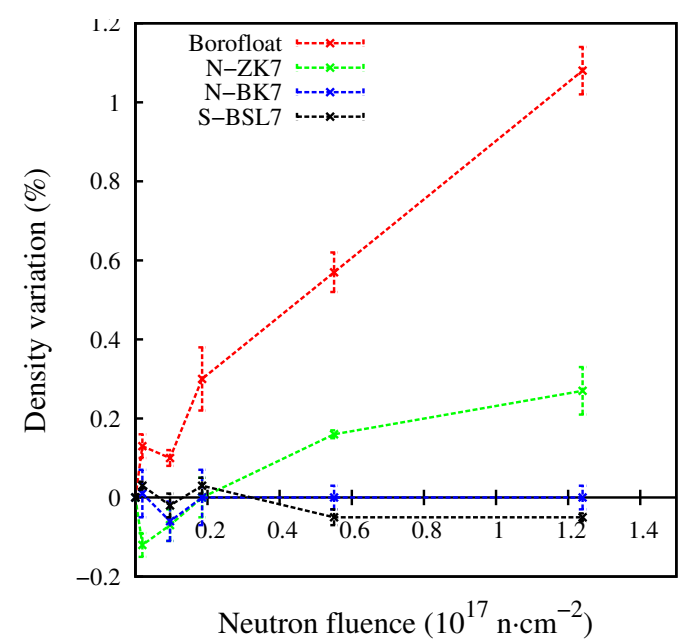

(a) Lines drawn between the points to ease the reading.

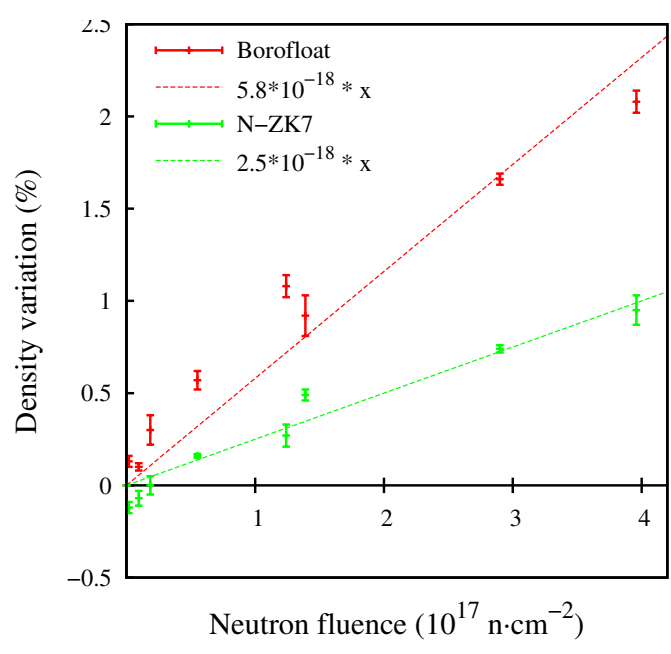

(b) Fitting of Borofloat and N-ZK7 datas at low fluence with a linear function.

Figure 3.12: Density variations at low fluence. Homogeneous neutron irradiation at a temperature below $65^{\circ} \mathrm{C}$.

\subsection{Stress generation in borosilicate glass by neutron irradiation}

The mechanical breakup of a solid body when exposed to a gradient of strain and stress is highly dependent upon both the object geometry and the loading conditions. As it has been referred to in the previous section, thin pieces of glass when put under a neutron flux, as homogeneous as practically achievable, may undergo a shrinking of their volume up to $2 \%$ without showing any sign of rupture. This was the case for Borofloat for a neutron fluence of $5 \times 10^{17} \mathrm{n} / \mathrm{cm}^{2}$ or higher. However, the same material cut into $10 \mathrm{~mm}$ thick pieces and subjected to grazing incidence radiation readily splinters at $2 \times 10^{15} \mathrm{n} / \mathrm{cm}^{2}$. The reason behind such different behaviours has to do with the generation of stress within the material due to the heterogeneous shrinking of the solid. In the first case, as the solid densifies homogeneously, no stress develops. In contrast, the grazing incidence conditions for this strongly absorbing material leads to the development of a depth dependent density gradient. Put into different words, the latter case could be visualized as if every horizontal layer within the material is bounded above and below by others with higher and lower density, respectively, which will result in the generation of compression and tensile stresses, respectively, for the middle layer. To try to ascertain a stress creation mechanism within our materials, an irradiation experiment was designed in order to combine the grazing incidence conditions with a thin sample that might be bent due to the stresses created.

\subsubsection{Experimental procedure}

The neutron irradiation was carried out using the Neutrograph test instrument [107]. A sample holder with a capacity of 8 glass plates has been designed for that purpose (see Fig 3.13a. The apparatus featured a neutron shield, made of $\mathrm{B}_{4} \mathrm{C}$ enriched in ${ }^{10} \mathrm{~B}$, on its 


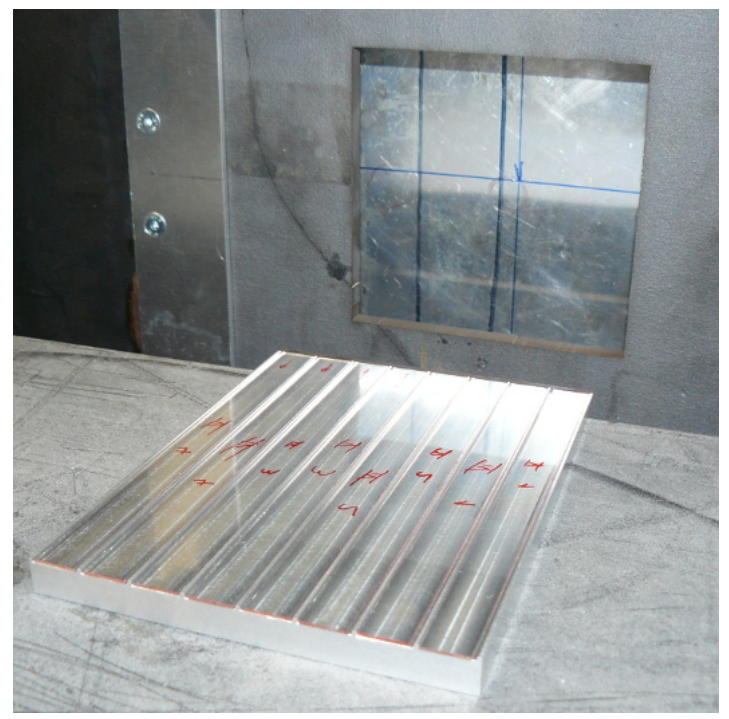

(a)

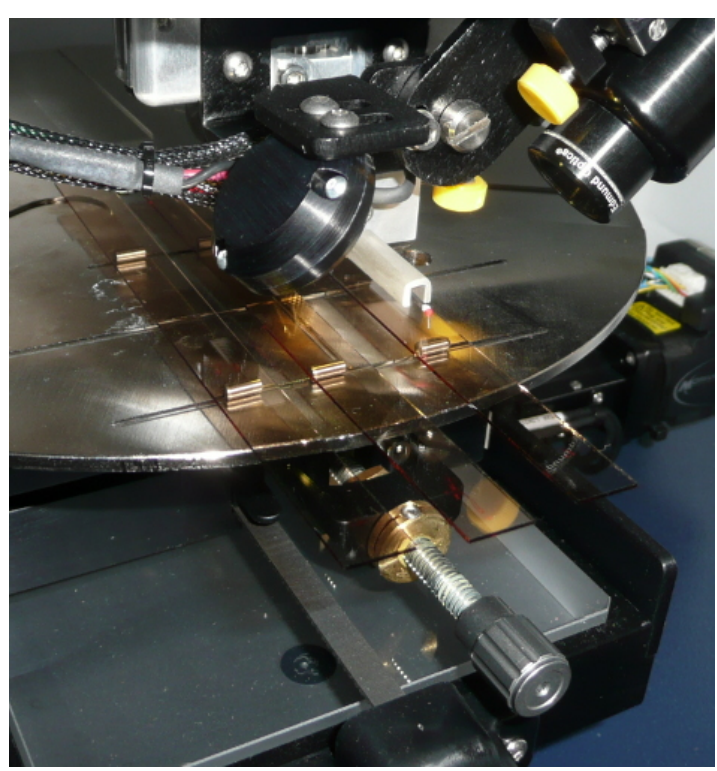

(b)

Figure 3.13: Borofloat strips set-ups for $2^{\circ}$ incidence thermal neutron irradiation, (a), and the subsequent curvature measurement, (b).

irradiated side to reduce its neutron activation and screen the front face of the samples. The Neutrograph is fed by the H9 thermal neutron beam, which has a $3 \times 10^{9} \mathrm{n} / \mathrm{cm}^{2} / \mathrm{s}$ thermal flux plus a fast neutron component of about $0.1 \%$. In view of the flux difference of orders of magnitude, the effects of the fast neutrons can in principle be disregarded. Also, is worth pointing out that the fast neutrons impinging on the glass plates will homogeneously modify the material, therefore not contribute significantly to the development of tensile stress; whereas, one expects that thermal and cold neutrons will generate the anisotropic phenomena under investigation here. The mean incidence angle of the neutrons was set to $2^{\circ}$. Such an angle is sufficient to avoid reflection of the neutrons at the glass surface, since their critical angle is approximately $0.11^{\circ}$ at thermal energy [67.

Only Borofloat glass strips were irradiated in these experiments. Their dimensions were $130 \mathrm{~mm} \times 10 \mathrm{~mm}$, with a thickness of $0.7 \mathrm{~mm}$. The selection of this unique material is motivated from two facts. First, Borofloat has been found to be the most reactive glass in terms of density variation and splintering, therefore it is expected to be showing large scale deformations. Second, Borofloat can be easily obtained in thin specimens, whereas the other glasses had to be polished and the neutron mirror manufacturers were not sure they would be able to achieve this for such a low thickness. Five batches of four glass strips were irradiated from 2 to 46 days.

Curvature measurements were performed with a Bruker Dektak XT-A stylus profiler. Glass strips were centred on two supporting cylinders, which were separated by a distance of $50.8 \mathrm{~mm}$. The load on the measuring tip was set to $3 \mathrm{mg}$, its minimum value. Figure $3.13 \mathrm{~b}$ shows three irradiated glass strips installed on the sample table. 


\subsubsection{Results}

Figure 3.14 displays the profile of one glass strip per received dose and the inverse of the averaged curvature over each sample group. The linear fit is set to be equal to zero for the pristine state. A look at to the plot just mentioned reveals the creation of significant curvature already achieved with the first dose, and then increases linearly as a function of the received fluence. We have chosen to plot the inverse curvature, instead of the curvature radius itself, as it is more physically relevant in terms of stress generation within the glass plate. Indeed, one can recall the pioneering study made by G. G. Stoney in 1909 where he determined the stress in the nickel coating of a steel rule [105]. The stress $(P)$ in the film is determined by,

$$
P=\frac{1}{6} E \frac{d^{2}+t d}{r t}
$$

where $E$ and $d$ are Young's modulus and thickness of the substrate, respectively, $t$ is the coating thickness, and $r$ is the measured curvature.

The measurements just reported thus prove the linear correlation between the neutron irradiation at low fluence and the generation of stress within the plate. This lends further support to the results of the previous section showing the density variation as a function of the neutron dose $(\mathrm{p} .71)$. It is also worth pointing out here that as in the H112/H21 experiments, one can reasonably expect that the increase of curvature might stop when the material reaches its cracking limit.

\subsubsection{Discussion}

\section{Analytical approach}

From the measurements reported in Fig. 3.14, one can try to extract values for the corresponding stress within the plate. The simplest way to do so would be to make use of the formula due to Stoney 105]. Our setup however is quite at variance from that considered by Stoney. Indeed, here, both strain and stress are occurring within the plate itself rather than being the result of the action of a coating process. As a first approximation, we could consider an equivalent "coating" layer that would correspond to the thickness where most of the absorptions occur, while the rest of the glass strip will be the equivalent "substrate". The Stoney formula is valid only when the "film" thickness is negligible with respect to the substrate thickness. In order to estimate a thickness, $t$, under the surface where most of the absorptions occur, one may compute the ratio of ${ }^{10} \mathrm{~B}(\mathrm{n}, \alpha)$ reactions above and below the depth $t$ to the irradiated surface.

$$
\frac{\int_{z=0}^{t} e^{-\frac{\Sigma_{\alpha} \cdot z}{\sin (\theta)}} \mathrm{d} z}{\int_{z=t}^{T} e^{-\frac{\Sigma_{\alpha \cdot z} \sin (\theta)}{\sin } \mathrm{d} z}} \approx e^{\frac{\sum_{\alpha \cdot t}}{\sin (\theta)}}\left(1-e^{-\frac{\Sigma_{\alpha} \cdot t}{\sin (\theta)}}\right)
$$

Where $\Sigma_{\alpha}$ is the macroscopic cross-section in the material of ${ }^{10} \mathrm{~B}(\mathrm{n}, \alpha)$ reactions, $\theta$ is the neutron mean incidence angle, and $T$ is the total sample thickness. In our case, consid- 


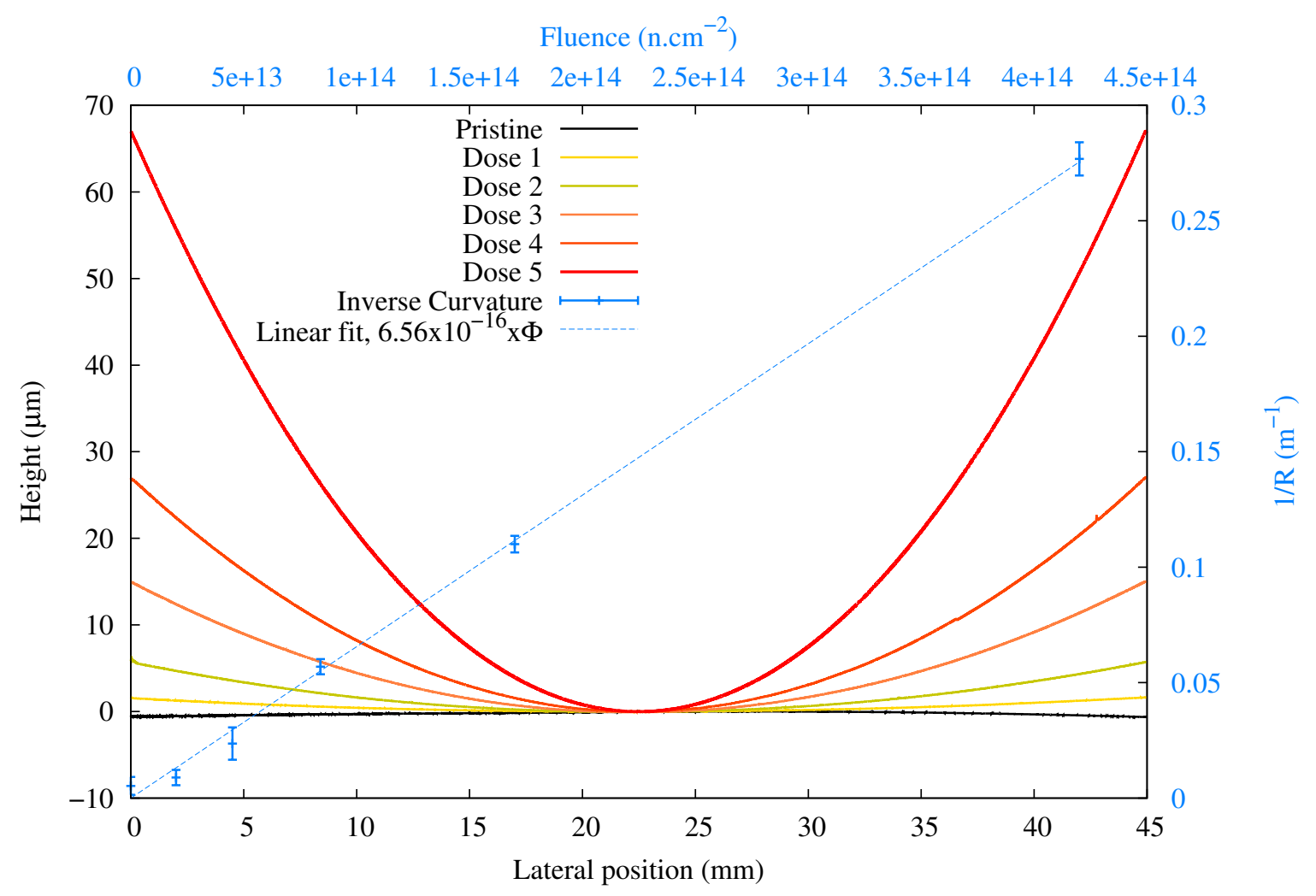

Figure 3.14

ering the mean incidence angle, $2^{\circ}$, the number of reactions at a depth of $0.7 \mathrm{~mm}$ can be neglected. For $t=0.16 \mathrm{~mm}$, this ratio is about 5 . In that case, $83 \%$ of the neutrons are absorbed within the first $0.16 \mathrm{~mm}$ under the surface. Given that the total thickness is $0.7 \mathrm{~mm}$, the thickness of the "stressed" layer is about 3 to 4 times thinner than the "unstressed" part. Though this may not be considered within the conditions were the Stoney formula can be applied, this is an easy way to obtain a rough estimate of the stress magnitude without an extensive numerical computation. Such an estimation gives for first and fifth fluence, a stress in the plate of $0.2 \mathrm{MPa}$ and $7 \mathrm{MPa}$, respectively. These values can be compared to the bending strength of Borofloat which is about $25 \mathrm{MPa} 97$.

\section{Numerical approach}

To further analyse the curvature measurements, we carried out a computational modelling with the software package ANSYS.

The homogeneous irradiations experiments have shown that Borofloat shrinks when irradiated by thermal neutrons. This microscopic behaviour induce stresses when occurring heterogeneously in the material. This explains why Borofloat strips are bent due to irradiation as it has been reported in Fig. 3.14. The aim of the computations presented here, is to try to connect, quantitatively, the shrinking of the glass under irradiation and the stresses it may induce in the case of an heterogeneous neutron flux in the material. 


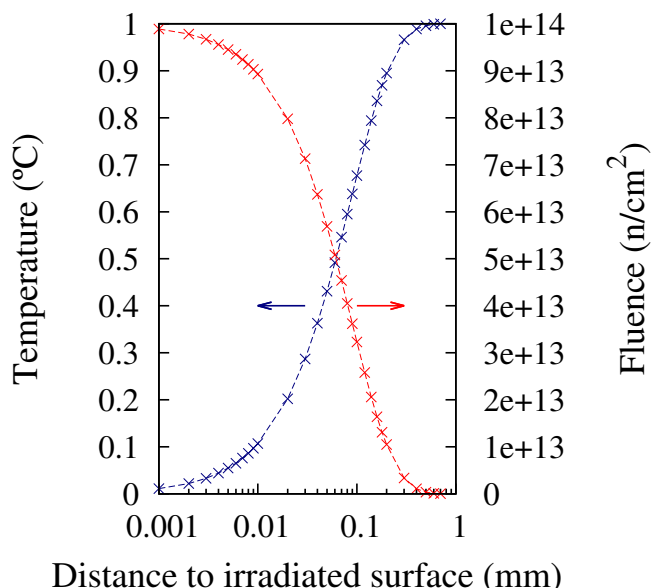

Figure 3.15: Fluence attenuation in the glass strip and $-1{ }^{\circ} \mathrm{C}$ gradient simulated in ANSYS.

To do so, a thermal analogue of the phenomena was used in ANSYS to try to mimic the compaction of the glass. A gradient of temperature was set in order to provoke an heterogeneous shrinking of the material through the thermal expansion coefficient. The sample geometry was set to that of the real samples, namely $130 \times 10 \times 0.7 \mathrm{~mm}^{3}$. We have chosen to follow the compaction law derived from Fig $3.12 \mathrm{~b}$ that yields a coefficient of $\frac{\Delta \rho / \rho}{n / \mathrm{cm}^{2}}=9 \times 10^{-20}$. As the mass variation due to neutron irradiation can be neglected in this fluence range, this latter coefficient is equivalent to a volume variation of $\frac{\Delta V / V}{n / \mathrm{cm}^{2}}=-3 \times 10^{-20}$. From that, an equivalent thermal dilation coefficient was derived so that a gradient of $-1^{\circ} \mathrm{C}$ is equivalent, in term of induced compaction, to a fluence of $1 \times 10^{14} \mathrm{n} / \mathrm{cm}^{2}$. The thermal dilatation coefficient was hence set to $3 \times 10^{-6}{ }^{\circ} \mathrm{C}^{-1}$. The isotropic elastic modulus and Poisson ratio were set to $64 \mathrm{GPa}$ and 0.2 , respectively, as it is reported on Borofloat manufacturer's website [97].

Figure 3.15 shows, in red, the fluence attenuation as a function of the depth in the sample, for an incident value of $1 \times 10^{14} \mathrm{n} / \mathrm{cm}^{2}$. Also on the same figure, in blue, the $-1^{\circ} \mathrm{C}$ gradient that should model the shrinking effect in the glass. Only the crossed points were considered in the ensuing calculations. It must be mentioned that no thermal conductivity properties were set for the material in order to ensure that the heat gradient is not perturbed.

The hexahedral mesh dimensions along the plate width $(10 \mathrm{~mm})$ and height $(130 \mathrm{~mm})$ were set both to $2 \mathrm{~mm}$. Its size was adjusted over the glass thickness, depending upon the thermal gradient. That is, large volume elements were considered on the back side of the sample where radiation effects were not large, whereas a thin mesh was used to account for the irradiated side. In real numbers, the employed elements were set to $0.05 \mathrm{~mm}$ elements with an gradual transition to smaller values modulated by a coefficient of 1.2 so that those elements touching the irradiated surface had a thickness of about $0.004 \mathrm{~mm}$. Some tests were also carried out using finer meshes but this significantly complicates the finding of a numerical solution. The simulation was run for two configurations, which parameters are gathered in Table 3.9.

Figure 3.16 shows the main results of the computations: curvature profiles along the 


\begin{tabular}{cc|ccc}
\hline \multicolumn{2}{c|}{ Input parameters } & \multicolumn{3}{c}{ Computation results } \\
$\begin{array}{c}\text { Maximum } \\
\text { contraction }\end{array}$ & $\begin{array}{c}\text { Temperature } \\
\text { gradient }\left({ }^{\circ} \mathrm{C}\right)\end{array}$ & $\begin{array}{c}\text { Maximum } \\
\text { stress }(\mathrm{MPa})\end{array}$ & $\begin{array}{c}\text { Maximum } \\
\text { deflection }(\mu \mathrm{m})\end{array}$ & $\begin{array}{c}\text { Inverse curvature } \\
\text { radius }\left(\mathrm{m}^{-1}\right)\end{array}$ \\
\hline $3 \times 10^{-6}$ & -1 & 0.15 & 5.2 & 0.0025 \\
$12 \times 10^{-6}$ & -4 & 0.6 & 20.6 & 0.0098 \\
\hline
\end{tabular}

Table 3.9: ANSYS input parameters and results. The temperature gradient follows the shape in Fig. 3.15, and thermal dilatation coefficient was set equal to $3 \times 10^{-6}{ }^{\circ} \mathrm{C}^{-1}$.

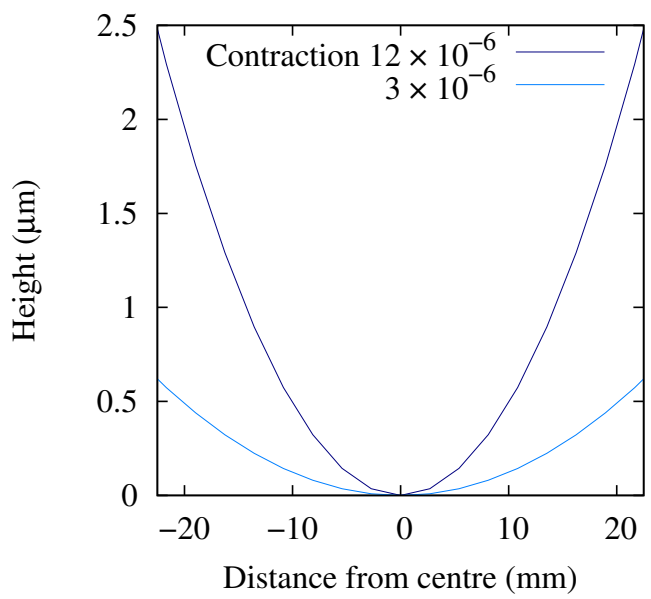

(a) Height profile of the irradiated surface.

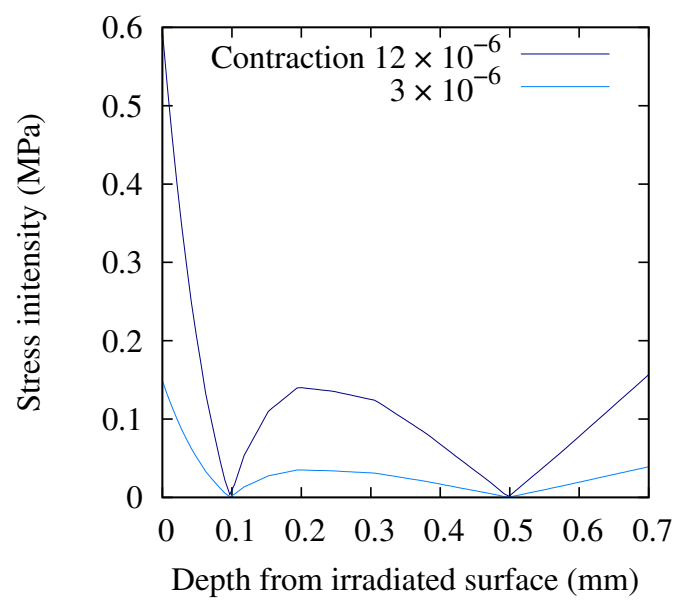

(b) Stress intensity accross the sample.

Figure 3.16: Result of ANSYS simulation with $-1{ }^{\circ} \mathrm{C}$ and $-4{ }^{\circ} \mathrm{C}$ temperature gradients, equivalent to contraction gradients of $3 \times 10^{-6}$ and $12 \times 10^{-6}$, respectively. Thermal dilatation coefficient set to $3 \times 10^{-6}{ }^{\circ} \mathrm{C}^{-1}$.

irradiated surface and stress intensity across the sample. The inverse curvature radius derived from the simulation is reported in Table 3.9. The maximum deflection quoted in the table refers to the height at the sample tip, $65 \mathrm{~mm}$ from the centre. The radius of curvature is derived thanks to this formula: $R=\frac{l^{2}}{8 \times x}$; where $l$ is sample's length and $x$ is the maximum deflection. This formula is valid if $R \gg l$, which is our case.

One can see from the comparison of Fig 3.14 and Fig, 3.16 a that the measured curvatures are much higher than the one simulated. Indeed, considering the contraction coefficient derived from the homogeneous irradiation, the simulated case that uses a maximum contraction of $3 \times 10^{-6}$ is supposed to give a similar curvature than the measured case around $1 \times 10^{14} \mathrm{n} / \mathrm{cm}^{2}$. Looking at the values for this case, the measurement yields an inverse radius around $0.066 \mathrm{~m}^{-1}$, while it is around $0.0025 \mathrm{~m}^{-1}$ for the simulation. That latter value is around 27 times too low. For the second configuration, the simulated the results are also too low and with the same factor of difference. This discrepancy might be explained by the fact that the contraction law has been derived around a fluence which is three orders of magnitude above which the grazing irradiation is done. Hence, this supports the idea that Borofloat contraction is not linear with the fluence when considering different orders of magnitude. 
Considering the stress intensity, one can see that it reaches, as expected, its maximum value within the first microns of depth. It yields a value around $0.6 \mathrm{MPa}$ for the second configuration that was supposed to mimic the $4 \times 10^{14} \mathrm{n} / \mathrm{cm}^{2}$ fluence. Such a value is noticeably below the one derived by the analytical calculation with Stoney's formula. The main reason for this being that, as it was seen with the curvature profiles, the contraction was under-estimated at this fluence.

This experiment of measuring plate bending due to irradiation allowed us to have a visual and quantitative measure of stress generation in glass plate due to irradiation. Of course, it highlights the need of new experiments of neutron ageing: density monitoring under homogeneous flux at lower fluence, or curvature monitoring under grazing incidence at higher fluence. The first solution will need a density measuring technique more accurate, while the second one will have to account the mean incidence angle modification due to the higher curvature of the sample. 


\section{Chapter 4}

\section{Vitreous structure analysis and response to thermal neutron irradiation}

\subsection{RAMAN spectroscopy}

\subsubsection{Brief notions of light scattering from amorphous struc- tures}

Glasses, while devoid of global periodicity in their macroscopic structure, are composed by structural units which show, at least on certain length-scales, a significant degree of order. The structure of most silicate glasses hinges upon some well defined microscopic units, such are the $\mathrm{SiO}_{4}$ tetrahedra which are connected together. Such well defined units which exist as a consequence of the formation of strong chemical bonds established between the atoms constituting the glass, set a characteristic scale also known as short-range-order, or SRO. It typically comprises some few tenths of a nanometre. At such scales, inelastic scattering of light by elementary excitations, such as the fundamental vibrations of the structural units forming the glass can be studied by means of the Raman effect which, up to a first order, involves the creation or annihilation of a vibrational excitation and obeys the rules,

$$
\begin{aligned}
& \omega_{i}=\omega_{s} \pm \Omega \\
& \mathbf{k}_{\mathbf{i}}=\mathbf{k}_{\mathbf{s}} \pm \mathbf{K}
\end{aligned}
$$

where the quantities with subscript $i$ stand for the frequency and wave vector of the incident light and $\Omega, \mathbf{K}$ refer to a vibrational excitation which is either created $(+)$ or annihilated (-) by the incoming light field. The former are known as Stokes processes whereas the latter are labelled as anti-Stokes processes. The intensity of such processes is temperature dependent through the occupation number of such excitations, which is given by Bose-Einstein statistics as $n_{k}=\left(\exp \left(\hbar \Omega / k_{B} T\right)-1\right)^{-1}$. Typical frequencies for the kind of processes considered here may vary widely depending upon the particular chemical structure of a given glass. They are usually above those of their crystal counterparts that are dominated by excitations of acoustic character, as briefly commented on below. 
For length-scales comprising, say, $0.5 \mathrm{~nm}$ to $2 \mathrm{~nm}$ or so, the structure of a material in its glassy state, such as vitreous silica, significantly differs from that shown by its crystalline variants. Such scale known as medium-range-order, or MRO, can also be accessed by Raman scattering, which in general can be described in terms of its frequencydependent intensity,

$$
I(\omega)=C(\omega) g(\omega)\left[n_{K}+1\right] / \omega
$$

given in terms of the density of vibrational states $g(\omega)$ and a light-to-vibrations coupling function $C(\omega)$. The former quantity is easily accessible from inelastic neutron scattering, whereas the latter needs to be specified for each glass, although several simplified expressions for this quantity have been given. The range of frequencies where such low-frequency Raman scattering is observed is typically bound below some 5 - $10 \mathrm{THz}$ (i.e. below some 20 - $40 \mathrm{meV}$ ). The spectra is usually dominated by a broad band, which broadens as the temperature is increased and finally merges which the quasi-elastic (Rayleigh) response from the glass.

For still larger scales, the response of a glass to the incident light is dominated by inelastic scattering processes corresponding to frequency shifts of the incident light, which are generated by coherent scattering by propagating vibrational motions caused by elastic waves (phonons). Such scattering processes, which typically have characteristic wavelengths of the order of $200 \mathrm{~nm}$ or so, take place within the $\mathrm{GHz}$ range and therefore probe the glass as a continuum able to sustain longitudinal and transverse acoustic excitations.

In what follows, we will concentrate our study on the effects of neutron radiation in glasses at frequencies characteristic of SRO, or above $9 \mathrm{THz}$ or so $\left(\approx 300 \mathrm{~cm}^{-1}\right)$, and look for detectable changes in spectra which can be attributed to differences in chemical bindings. This does not rule out that significant changes associated with the elastic properties can also take place at MRO and longer-range scales. However, since both kinds of experiments require either a dedicated low-frequency spectrometer or an optical interferometer, we leave this issue for studies beyond the realm of the current Ph.D. thesis.

\subsubsection{Raman signals on pristine glasses}

The measurements reported within the present study have been made on a LabRAM HR800 machine from Horiba, using a green laser as the excitation source with a wavelength of $532 \mathrm{~nm}$. The experiment was done at the Commmissariat à l'Énergie Atomique at Marcoule, and specifically the setup was operated within the ATALANTE facility. Figure 4.1 shows the Raman spectra of the 4 glasses of interest. The full frequency range explored can be split into sub parts corresponding to motions pertaining specific structural units. In a nutshell,

From 300 to $550 \mathrm{~cm}^{-1}$ in Fig. $4.2 \mathrm{a}$ is usually assigned to bond-angle bending and stretching of Si-O-Si units [27]. Such a large band drifts towards higher frequencies for S-BSL7 and N-BK7. This was correlated to the increase of alkali content in the chemical composition 69,72]. In our case, the shift also correlates with alkali concentration, with N-BK7 and S-BSL7 having the highest sodium and potassium 


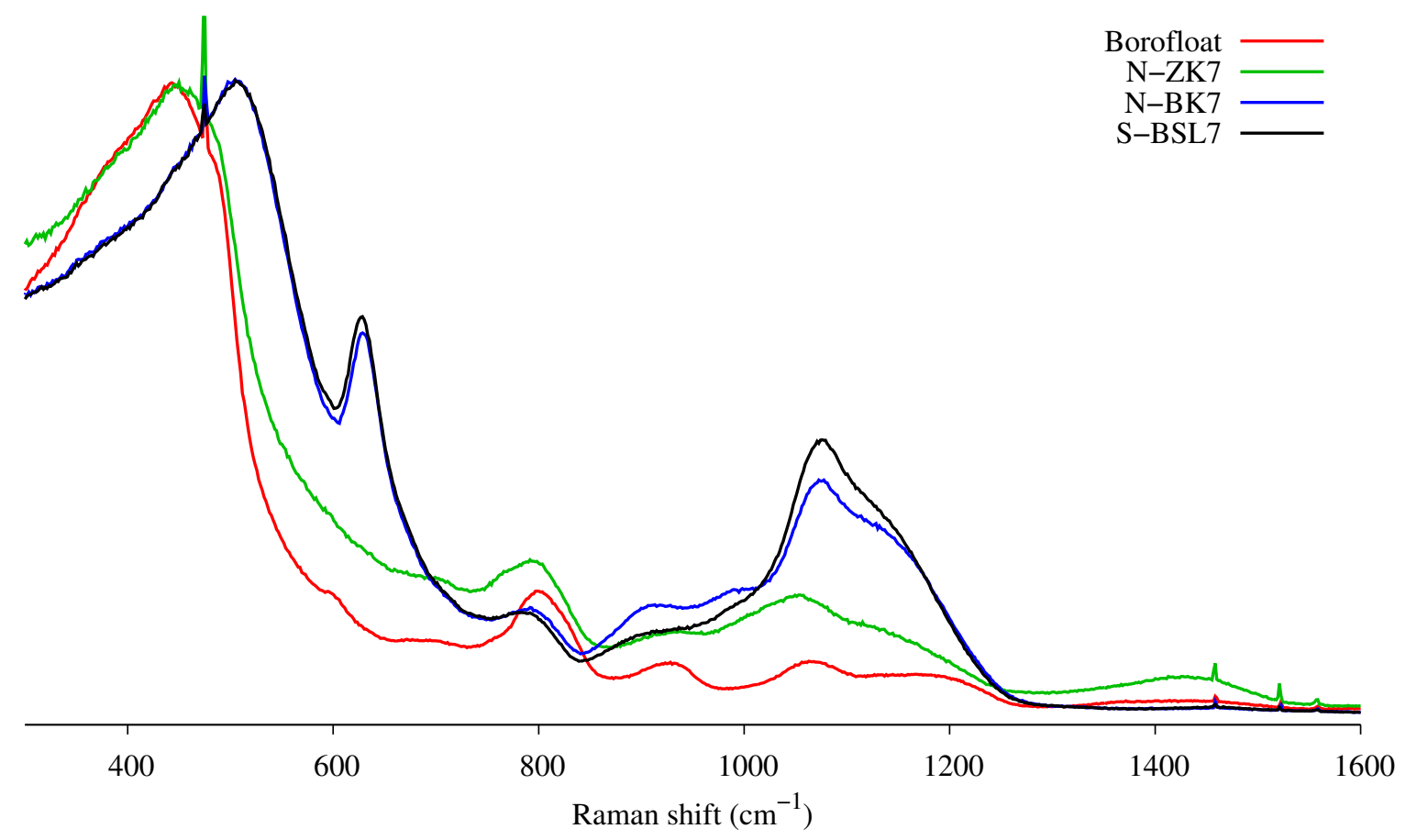

Figure 4.1: Raman spectra of the four glasses in their pristine state. The intensity of each curve is arbitrary.

content. Such a feature is characteristic from ABS glass with a mixing of borate and silicate units.

From 550 to $850 \mathrm{~cm}^{-1}$ in Fig. $4.2 \mathrm{~b}$ is generally assigned to breathing modes of rings forming the borate or borosilicate groups [74]. The band about $808 \mathrm{~cm}^{-1}$ has been assigned to three-coordinated boron in boroxol rings. Another band around $770 \mathrm{~cm}^{-1}$ is attributed to four coordinated boron in diborate units [69]. Notice that the relative strength of the $806 \mathrm{~cm}^{-1}$ band is higher in Borofloat than in the three other glasses, which could lead one to conclude that this material has more 3-coordinated boron atoms, resulting in to a lesser-homogeneous material.

A distinctive feature of spectra for N-BK7 and S-BSL7 also assigned to the borosilicate network shows up as a relatively narrow peak, centred at $630 \mathrm{~cm}^{-1}$. Its origin has been suggested to be the four-coordinated boron atoms, which could infiltrate the silica network and generate reedmergnerite-like units [119. However, this was later counter-balanced by Bunker et al. [27], who thought that this band could be created by danburite-like units. Such a structure needs one additional 4-coordinated boron compared to reedmergnerite. The advantage of danburite is that its crystalline Raman peak is measured at $614 \mathrm{~cm}^{-1}$ compared to $586 \mathrm{~cm}^{-1}$ for the reedmergnerite. Even though the contributors to this band are not well defined, its presence proves an intermixing of the borate and silicate networks.

Finally, a weak peak around $600 \mathrm{~cm}^{-1}$ is visible in the Borofloat spectrum but has not been assigned in the literature, yet. One might think that it could be generated by pure reedmergnerite units. 
From 850 to $1300 \mathrm{~cm}^{-1}$ in Fig $4.2 \mathrm{c}$ is assigned to stretching of the Si-O, bond with different contributions depending on the number of bridging oxygen of the silicon tetrahedra. Manara et al. [69] divide this large band into five contributions, peaking at $910 \mathrm{~cm}^{-1}$ for $Q_{1}, 960 \mathrm{~cm}^{-1}$ for $Q_{2}, 1060 \mathrm{~cm}^{-1}$ for $Q_{3}, 1150 \mathrm{~cm}^{-1}$ for $Q_{4}$, and an additional gaussian centered around $1020 \mathrm{~cm}^{-1}$. More reliable information is, however, provided by the NMR measurements concerning the short range order surrounding silicon. Notice that the electro/optical parameters governing the Raman intensity rules tell us that such a band will exhibit different features depending upon whether it is a $\mathrm{Si}-\mathrm{O}-\mathrm{Si}$ or a $\mathrm{Si}-\mathrm{O}-\mathrm{B}$, which is vibrating.

From 1300 to $1600 \mathrm{~cm}^{-1}$ in Fig. $4.2 \mathrm{~d}$ has been assigned to the stretching of B-Obetween $\mathrm{BO}_{3}$ and $\mathrm{BO}_{4}$ for a band around $1410 \mathrm{~cm}^{-1}$ and between $\mathrm{BO}_{3}$ and $\mathrm{BO}_{3}$ for a band near $1480 \mathrm{~cm}^{-1}$ [69]. Notice, however, that this is a region with rather weak intensity superposed to large background noise, and this makes the analysis tentative at best. However, one could note a lower contribution of the $\mathrm{BO}_{3}-\mathrm{BO}_{4}$ band for the N-BK7 and S-BSL7 glasses. This could be attributed to a better integration of the four-coordinated borons in the silica network and validate the existence of reedmergnerite and/or danburite units.

The last point that should be mentioned concerning these Raman spectra is the existence of crystalline peaks at 473, 1458, 1520, and $1559 \mathrm{~cm}^{-1}$. Several contributors have been suggested coming from the elements naturally contained in the glasses : albite $\left(\mathrm{NaAlSi}_{3} \mathrm{O}_{8}\right)$, poudretteite $\left(\mathrm{KNa}_{2}\left(\mathrm{~B}_{3} \mathrm{Si}_{12}\right) \mathrm{O}_{30}\right)$, and quartz $\left(\mathrm{SiO}_{2}\right)$. Two additional possibilities due to the Cerium Oxide polishing were considered: cerianite $\left(\mathrm{CeO}_{2}\right)$ and stillwellite $\left(\mathrm{CeBSiO}_{5}\right)$. The Raman curves of the mentioned compounds in their crystalline state have been plotted on the Fig. 4.3 between 350 and $650 \mathrm{~cm}^{-1}$. The different spectra were found on the website of the RRUFF Project [2].

Even though none of the suggested crystals match with the $473 \mathrm{~cm}^{-1}$ peak, further research will be carried out to try to find the chemical compound corresponding to the detected signals.

\subsubsection{Irradiated specimens}

\section{Experimental procedure}

The irradiated specimens were $1.1 \mathrm{~mm}$ thick and had been irradiated at the T4 facility. To avoid any contamination of the probed surface, each tile was broken by hand and the Raman analysis was done on the freshly fractured face. To allow measurement of the irradiated samples, an optical microscope $(100 \times$ objective) within a hot cell was employed, which was coupled to the spectrometer with an optical fiber. Here, we report on results concerning samples which received three neutron doses, which correspond to those of $1^{\text {st }}, 3^{\text {rd }}$, and $5^{\text {th }}$ from Table $3.8 \mathrm{p} .68$, named F1, F3, and F5, respectively.

To derive the shift of the R-band from the Raman signals, data were treated with LabSpec software. The processing consisted in removing the luminescent signal, smoothing the curve to attenuate the noise, and making a first order derivative. To remove the signal rising from luminescence, the high frequency ramp was fitted with a straight line and was subtracted on the spectra. The resulting curve was then smoothed to increase the signalto-noise ratio. For that purpose, the Savitsky-Golay method was used. The polynomial 


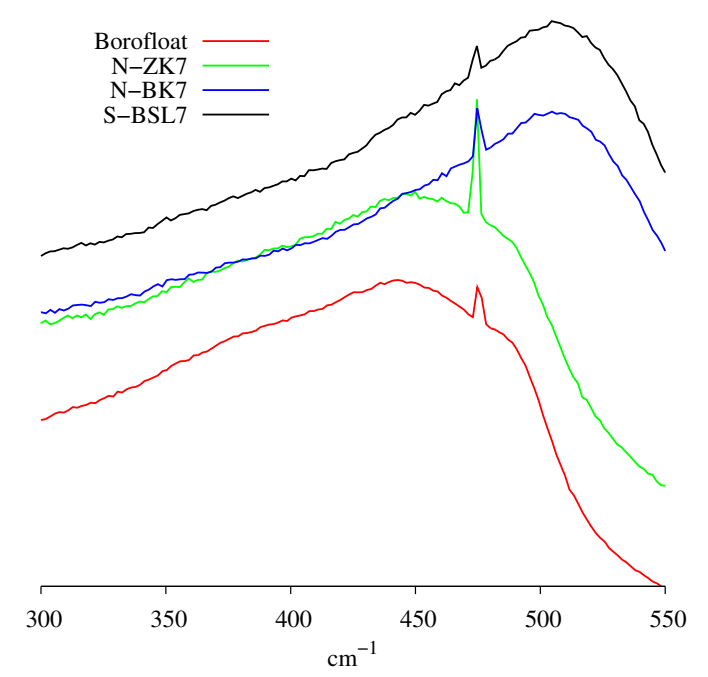

(a) Frequency band characteristic of Si-OSi elements.

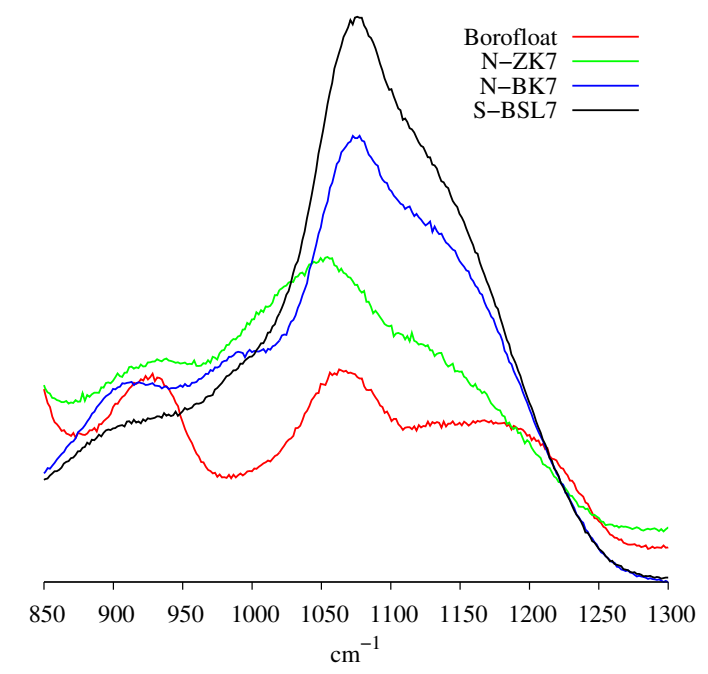

(c) Frequency band attributed to the different $Q_{n}$ signals.

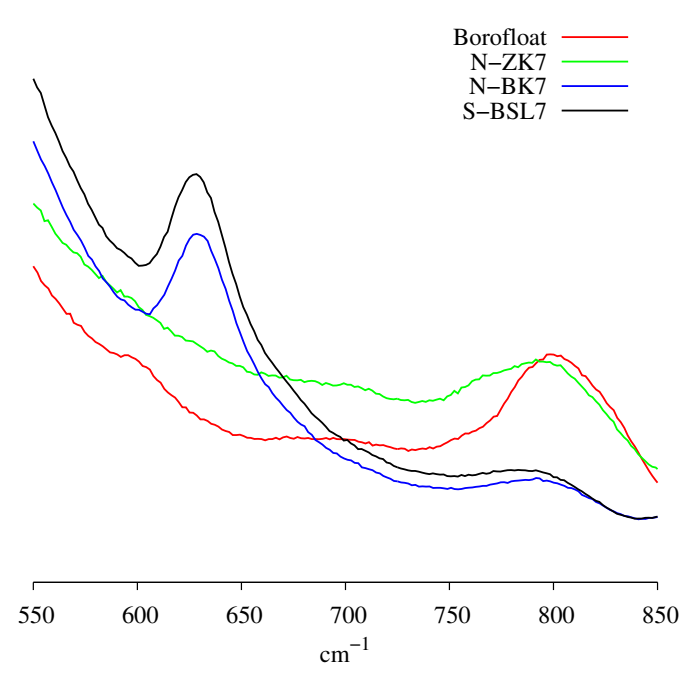

(b) Frequency band linked to borate and borosilicate structural units.

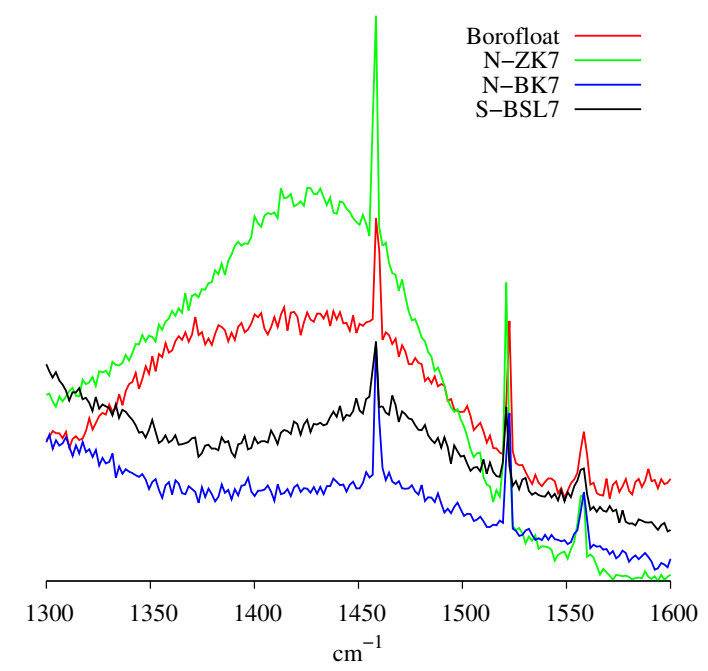

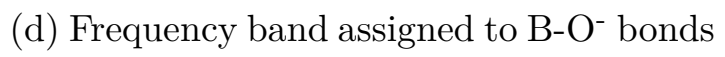
stretching.

Figure 4.2: Magnifications of the Raman spectra of Fig. 4.1 at the different frequency bands of interest. The intensity of each curve is arbitrary from one material and figure to the other. 


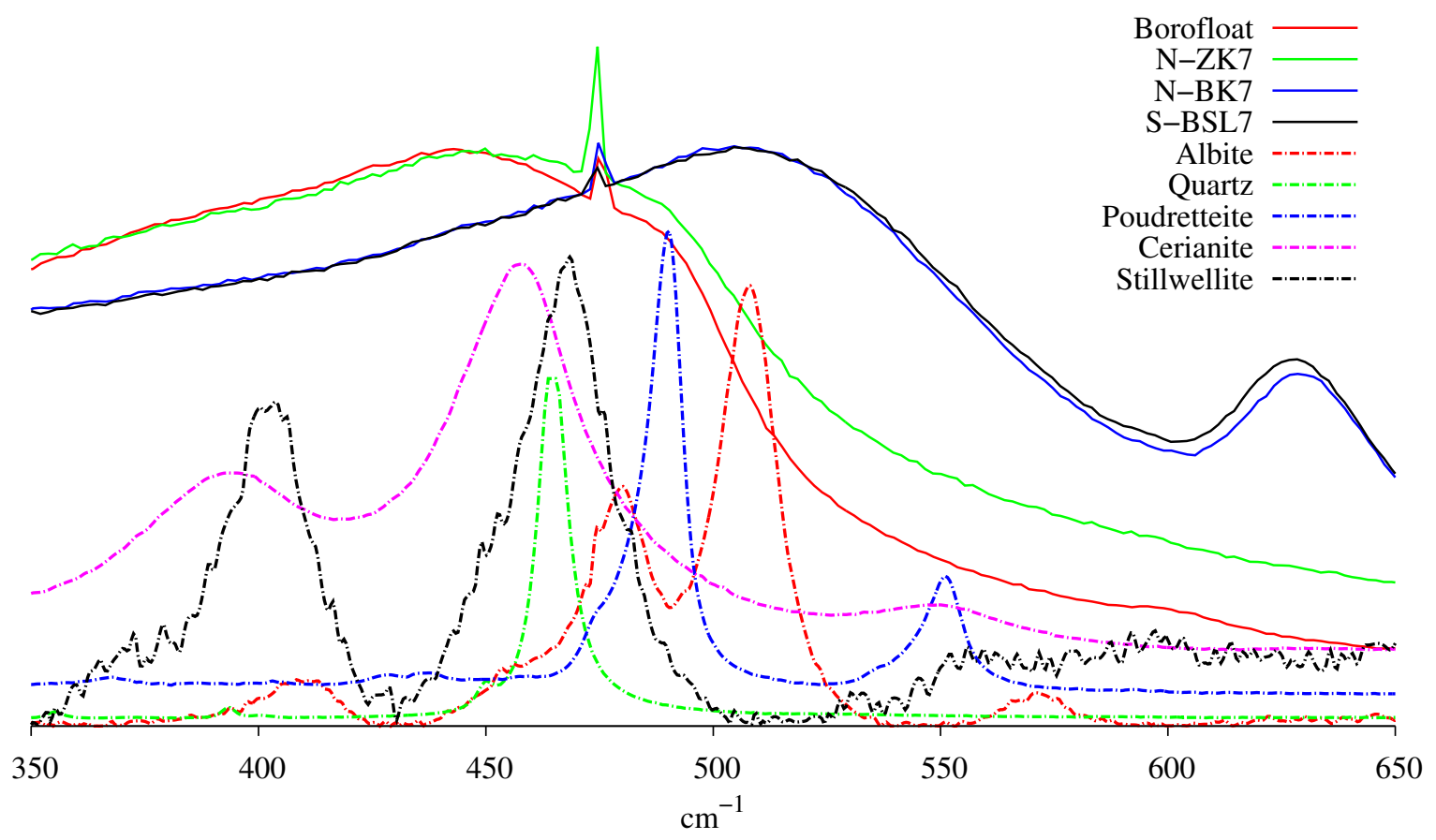

Figure 4.3: Raman spectra of the four glasses in their pristine state. The intensity of each curve is arbitrary.

used for the fitting was of degree 2 with 9 data points around the sliding analysed position. Once the derivative had been computed, the position of the R-band was inferred from the point at which it changed signs. Because of the noise remaining after smoothing, care must be taken since it may lead to some inaccuracy on the band position resulting from oscillations of the first order derivative.

\section{Results}

The Raman spectra of irradiated samples develop, in most cases, a strong luminescent background resulting from radiation-induced colour centers that make quantitative interpretation of spectra difficult, especially for frequencies above $1000 \mathrm{~cm}^{-1}$. It is for that reason that no signal could be measured on N-BK7. However, it has been possible to extract useful information from features appearing at lower Raman shifts. Spectra of Borofloat, N-ZK7, and S-BSL7 are shown in Fig. 4.4a, 4.4b, and 4.4c, respectively.

The significant background noise over the whole bandwidth comes from the signal losses in the components (mirrors, connectors, optical fibre, etc.) between the microscope situated in the hot cell and the spectrometer located outside. Borofloat and N-ZK7 Raman spectra evolve in a similar way as a function of the dose. The Si-O-Si band is shifted toward higher frequencies, and we can notice a growth of the D2-band around $600 \mathrm{~cm}^{-1}$ usually assigned to three-membered silicon rings [14]. Also, the boroxol $\left(\simeq 800 \mathrm{~cm}^{-1}\right)$ and diborate $\left(\simeq 770 \mathrm{~cm}^{-1}\right)$ bands lose intensity with respect to the rest of the spectrum. For S-BSL7, the Si-O-Si band does not show large changes, except at F5 where it is shifted about $10 \mathrm{~cm}^{-1}$. One could also notice a broadening of the $630 \mathrm{~cm}^{-1}$ peak and of 
the shoulder around $600 \mathrm{~cm}^{-1}$ that could be associated to an increase in concentration of three-membered rings.

\section{Discussion}

Let us first consider S-BSL7. One can see that the spectral features are not largely affected by radiation, with the exception of the luminescent background which becomes rather strong above $1300 \mathrm{~cm}^{-1}$. The $630 \mathrm{~cm}^{-1}$ peak, associated with reedmergnerite/danburite units, is almost unaffected for samples which have received the first and third doses but becomes more significant for the fifth dose. Its broadening could be associated with the growth of the D2-band, which is often related to three-membered silicon rings. This effect has already been observed on a similar sodium borosilicate glass irradiated by the ${ }^{10} \mathrm{~B}(\mathrm{n}, \alpha){ }^{7} \mathrm{Li}$ reaction 82 . In parallel to this D2-band increase, a shift of the $\mathrm{R}$-band of about $12 \mathrm{~cm}^{-1}$ was observed by the authors, together with a swelling of around $1.5 \%$. S-BSL7 shows a similar swelling level at F5 irradiation dose and the $\mathrm{R}$ band seems to be shifted about $10 \mathrm{~cm}^{-1}$, see Fig. 4.5. This feature has been related by Furukawa and White 47 to the incorporation of boron in the silicon network, and could well be an indication of a conversion from danburite-like to reedmergnerite-like units.

In contrast, Borofloat and N-ZK7 are much more reactive, as can be inferred from changes in their density and Raman spectra. Indeed, the Raman shift of the Si-O-Si peak towards higher frequencies is about 5 times stronger than that of S-BSL7. Moreover, the density change versus dose seems to be correlated with the amplitude of the R-band. This displacement is particularly strong for doses 1 and 3, whereas it does not show further changes between doses 3 and 5, a behaviour which mimics that followed by the density. The first order derivative Raman curves are shown in Fig. 4.6a and 4.6b for Borofloat and N-ZK7, respectively, in their pristine state, and for F1 and F3. From those plots, it is possible to extract the R-band shift and plot it versus the glass shrinking parameter. This is done in Fig. 4.7. On the other hand, a glance to Fig. 4.6a will serve to gauge the difficulties of extracting precise spectral parameters for the irradiated samples. In fact, the frequency position of most bands can easily be estimated for the pristine state. However, things become more complicated for samples such as F1, and results in significant error bars as depicted in Fig. 4.7. Even so, the plot shows a linear correlation between the glass contraction and the R-band shift.

The noticeable growth of the D2-band with increasing radiation dose pinpoints an important re-arrangement of the silica network. In addition, the strength of the diborate and boroxol bands $\left(770 \mathrm{~cm}^{-1}\right.$ and $800 \mathrm{~cm}^{-1}$ respectively) seem to decrease with increasing dose. Considering the fact that the maximum R-band shift of pure vitreous silica under irradiation is around $30 \mathrm{~cm}^{-1}[35]$, the feature observed for those two glasses could be a combination of effects comprising the shrinking of the silica network together with mixing with boron units. In summary, these spectral features lend support to the idea that the behaviour of Borofloat and N-ZK7 under irradiation is mostly governed by the silica-rich domains that represent the main phase of such clustered glasses due to their high silicon content. The higher density variation of Borofloat (2\%), if compared to N- 


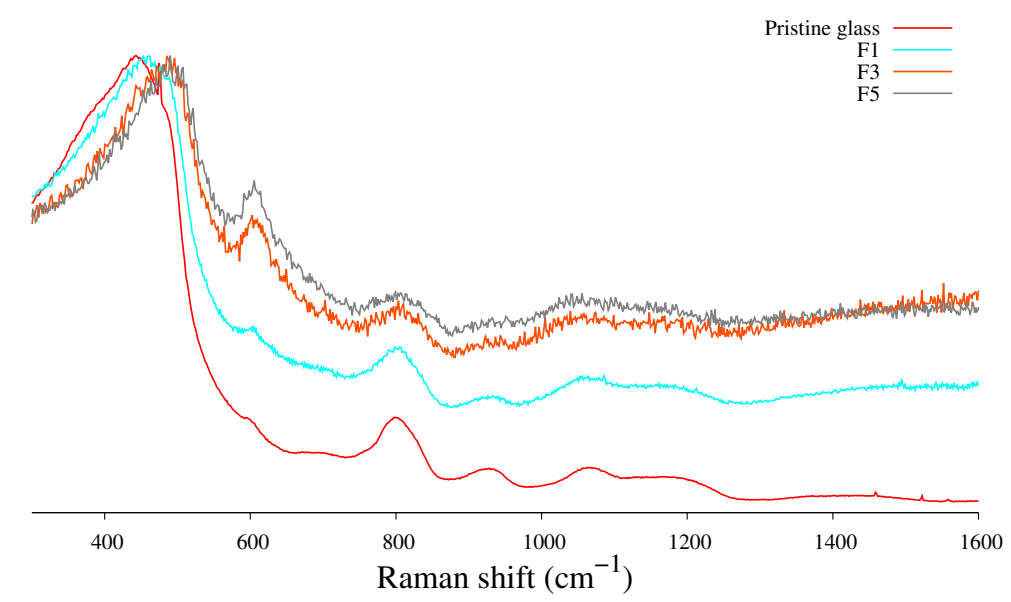

(a) Borofloat

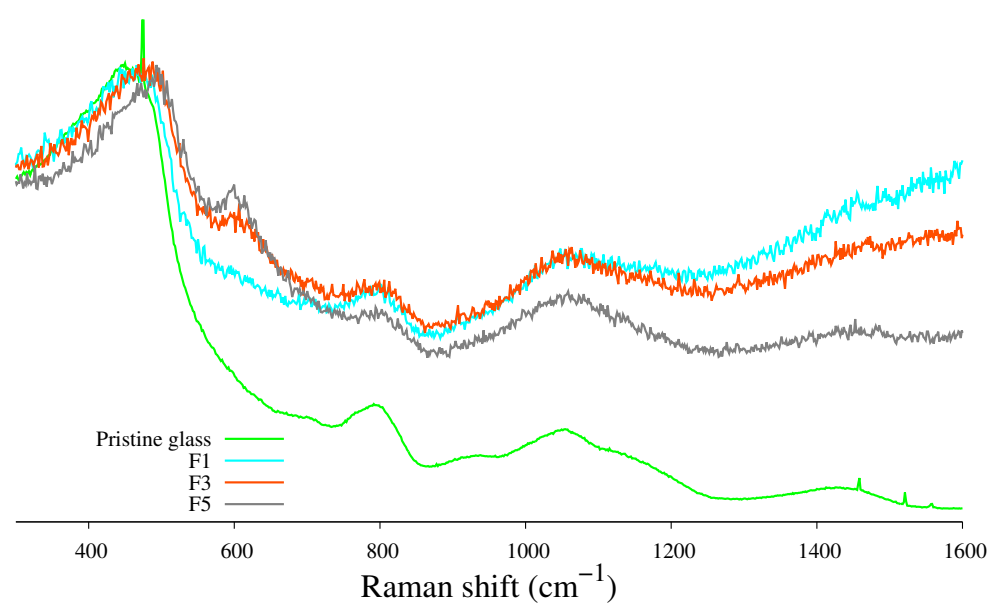

(b) N-ZK7

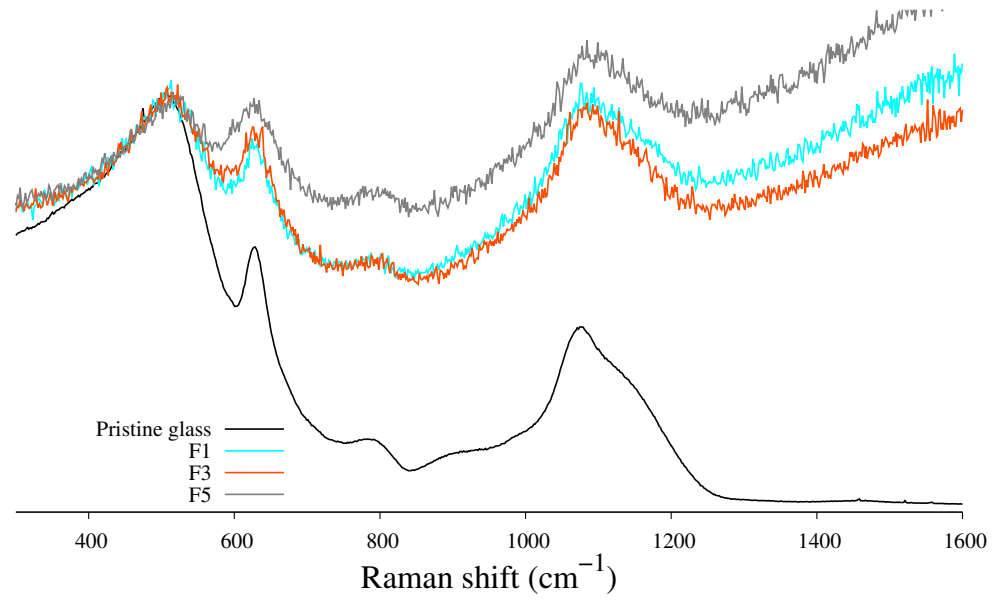

(c) S-BSL7

Figure 4.4: Raman measurements on irradiated samples plotted with the pristine spectrum. 


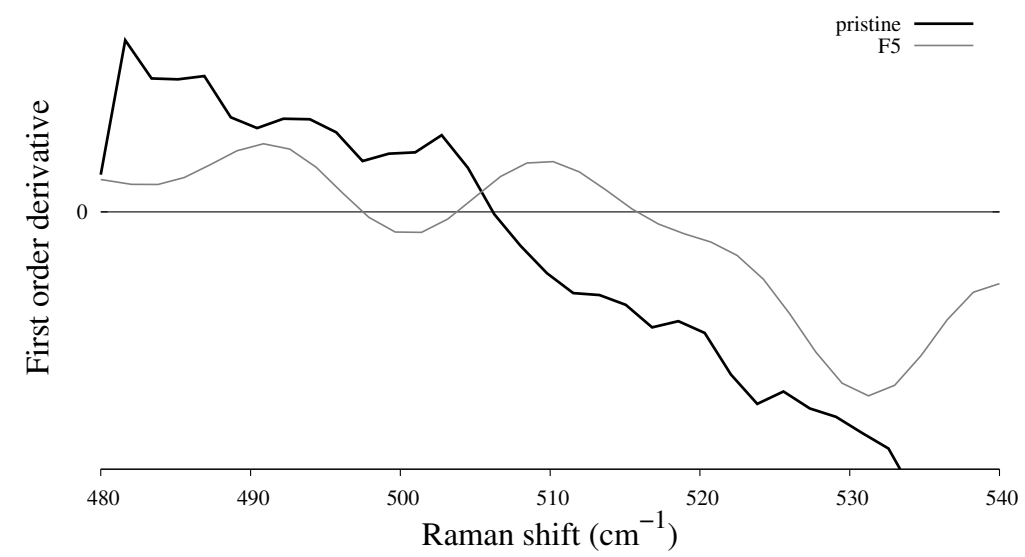

Figure 4.5: First order derivative of the Raman curve from SBSL-7 glass. Calculation done after subtraction of luminescent noise at high frequency and smoothing.

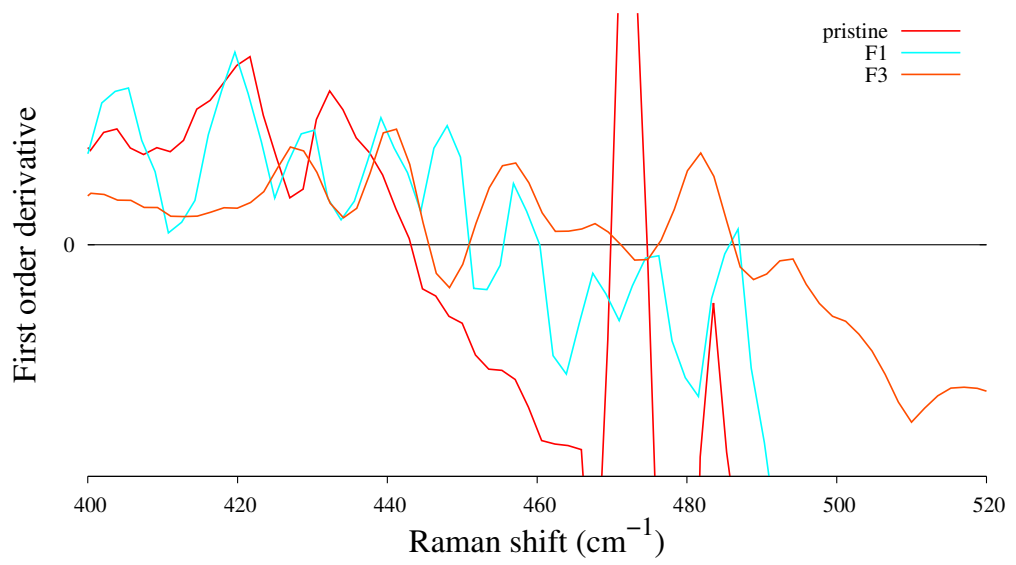

(a) Borofloat

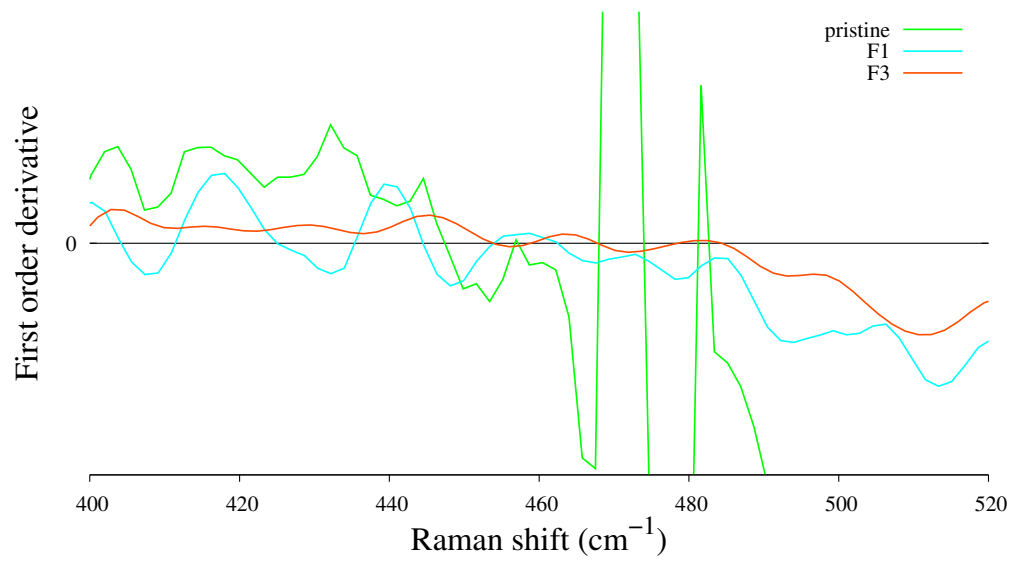

(b) N-ZK7

Figure 4.6: First order derivative of the Raman curve from Borofloat and N-ZK7 glass. Calculation done after subtraction of luminescent noise at high frequency and smoothing. 


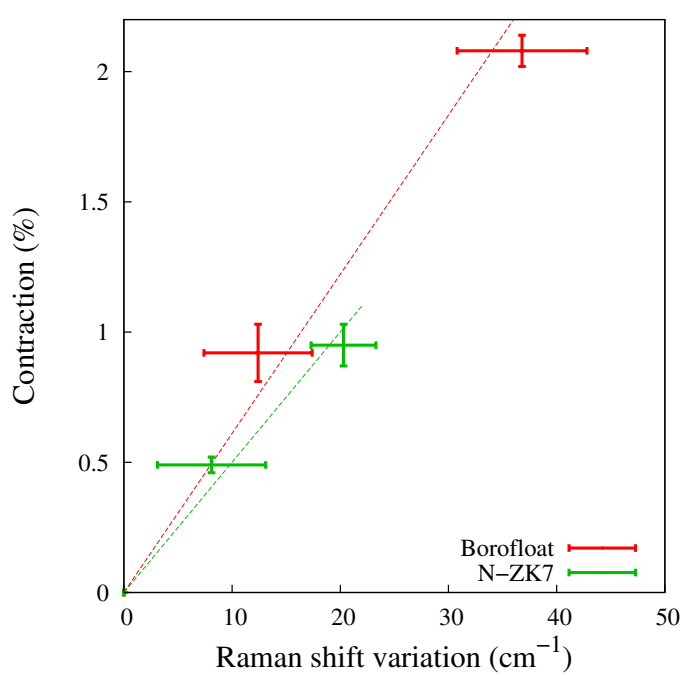

Figure 4.7: Density variation as a function of R-band position shift for doses 1 and 3

ZK7 (1\%), might be explained by its greater similarity with silica that shrinks up to $3 \%$ under irradiation [35]; Borofloat has $82 \%$ of $\mathrm{SiO}_{2}$, an R-band located around $445 \mathrm{~cm}^{-1}$ $\left(\sim 440 \mathrm{~cm}^{-1}\right.$ for vitreous $\left.\mathrm{SiO}_{2}\right)$, and even well identified clustering features (intense D2 and boroxol bands).

\subsection{NMR spectroscopy}

\subsubsection{Principles and techniques of NMR spectroscopy}

Apart from Raman characterization, the solid state Nuclear Magnetic Resonance technique has become, in the last decades, a powerful tool to look deeper into the glass structure [37], particularly to detect and quantify the elementary units that, when assembled, create the structures described above such as the boroxol ring, three-membered silicon rings, reedmergnerite-like units, diborate, etc. The method has several advantages over some other spectroscopies, among which its sensitivity to specific nuclei, the direct proportionality between the signal intensity and the nuclear specie population, and the straightforward sample preparation needed for an experiment are included.

This characterization method uses the fact that some nuclei have a sizeable magnetic moment which stems from the existence of nuclear angular momenta, also known as spin. The norm of its vector operator $\hat{I}$ and z-component $\hat{I}_{z}$ are quantified as,

$$
\begin{gathered}
|\hat{I}|=\sqrt{I(I+1)} \cdot \hbar \\
\left|\hat{I}_{z}\right|=m \hbar
\end{gathered}
$$

where $I$ is the spin quantum number of the nuclei and $m$ the orientational quantum 
number with $-I \leq m \leq I$. The magnetic moment $\mu_{z}$ of a given nucleus is linked to each spin state through a proportionality constant of scalar magnitude, known as the gyromagnetic ratio, $\gamma$, by the relation

$$
\mu_{z}=\gamma m \hbar
$$

The application of a magnetic field $B_{0}$ produces an interaction energy of the nuclear system, which amounts $-\mu B_{0}$. This leads to a very simple Hamiltonian, which in the case of the field to be directed along the z-direction can be expressed using a simple formula such as,

$$
H_{z}=-\mu_{z} B_{0}
$$

with eigenvalues multiples of $\gamma \hbar B_{0}$, which lead to energy levels that are equally spaced and separated by a value given by such a magnitude, that is

$$
E_{m}=-m \gamma \hbar B_{0}
$$

where $m=I, I-1, \ldots,-I$. For the simplest case of a half-integer spin, such as that of a hydrogen nucleus $(I=1 / 2)$, there are two distinct eigenstates. The jump between one state to another can be detected by applying electromagnetic waves that fulfils the Bohr condition: $\Delta E=\hbar \omega_{0}$. From that, one finds that the angular resonance frequency $\omega_{0}$, or Larmor Frequency, given by:

$$
\omega_{0}=\gamma B_{0}
$$

To induce transitions between the discrete energy levels the most commonly used setup usually consists in an alternating magnetic field applied perpendicular to the static external magnetic field. If we set the magnitude (or amplitude) of the alternating field to $B_{x}$, we get a perturbation term for the total Hamiltonian that comes to be of the form $H_{\text {pert }}=-\gamma \hbar H_{x} I_{x} \cos \omega t$. The operator $I_{x}$ has elements connecting spin states $m$ and $m^{\prime}$, which vanish unless $m^{\prime}=m \pm 1$. To derive an estimate of the order of magnitude of the energies involved in such spin transitions, one can rely on a fully classical analogy and consider a particle of mass $M$, which moves along a circular path of radius $r$ with period $T$. Its angular momentum is given by

$$
J=\frac{M 2 \pi r^{2}}{T}
$$

and its associated magnetic moment could be estimated from consideration of such a system as a loop of area $S$ that carries a current $i_{c}=(e / c)(1 / T)$, where $e$ stands for the electron charge and $c$ is the velocity of light. From here, we get that for such a classical system,

$$
\mu=\frac{e}{c} \frac{\pi r^{2}}{T}
$$

From here we get an estimate of $\gamma=\mu / J=e / 2 M c$. This tells us that $\gamma$ is inversely proportional to the mass, and thus we should expect that even for a light nucleus, $\gamma$ should be some thousand times smaller than the equivalent quantity for an electron, which is precisely known and amounts to $9.274 \times 10^{24} \mathrm{~J} / \mathrm{T}$ and referred to as the Bohr magneton. In consequence, whether electron systems for applied magnetic fields of the 


\begin{tabular}{ccccc}
\hline Nucleus & Spin & $\begin{array}{c}\text { Natural abundance } \\
(\%)\end{array}$ & $\begin{array}{c}\text { Larmor frequency } \\
\text { at 9.4 T (MHz) }\end{array}$ & Typical problems \\
\hline${ }^{11} \mathrm{~B}$ & $3 / 2$ & 80.42 & 128.4 & small range of $\delta_{\text {iso }}$ \\
${ }^{23} \mathrm{Na}$ & $3 / 2$ & 100 & 105.8 & lineshape interpretation \\
${ }^{27} \mathrm{Al}$ & $5 / 2$ & 100 & 104.3 & lineshape interpretation \\
${ }^{29} \mathrm{Si}$ & $1 / 2$ & 4.7 & 79.5 & Enrichment sometimes required \\
\hline
\end{tabular}

Table 4.1: Nuclear magnetic properties of the nuclei studied in this work.

order of 3-10 kG should resonate for applied frequencies of the order of $10 \mathrm{GHz}$, nuclear systems should show up at frequencies within the $\mathrm{MHz}$ range.

Some characteristics for nuclei of interest for our study are listed in Table 4.1. The resonance frequency is given for a $9.4 \mathrm{~T}$ magnetic field.

As the different eigenstates are not equally populated due to their difference of energy, the vector sum of their magnetic moment induces a macroscopic magnetization of the whole sample. Such magnetization which is a vector quantity should, in the absence of the perturbing field, be aligned with the applied static field, that is, along the z-axis. Its direction suddenly changes after a short pulse of the RF field which tilts its direction towards, say, the x-axis. The absorption of electromagnetic radiation is then detected through an a.c. voltage in the NMR probe coil. To have some values in mind, the radiofrequency pulse needed to flip the magnetization at $90^{\circ}$ from the magnetic field has to be short, from 1 to $10 \mu \mathrm{s}$, and intense, between 100 and $1000 \mathrm{~W}$ power. The decay of the a.c. voltage is recorded as a function of the time, and a Fourier-transformation allows us to derive the frequency domain of the spectrum.

The measured precession frequencies are dominated up to a first order by the Zeeman interaction, which is fully dependent on the nucleus itself. However, other parameters will influence them and will allow us to probe the glass structure itself. The local magnetic field $B_{l o c}$ induces a deviation (i.e. a chemical shift) from the Larmor frequency,

$$
\omega_{l} O c=\gamma B_{l o c}
$$

Among the different sources of perturbation one finds the magnetic interactions of the nuclei with the surrounding electrons (magnetic shielding interaction), the magnetic dipoledipole interactions among nuclei, and the interactions between the electric quadrupole moment of spin $>1 / 2$ nuclei with the electrostatic field gradients surrounding these nuclei (nuclear electric quadrupolar coupling).

Finally, it is worth mentioning the existence of two techniques especially relevant for the study of samples in the solid state which are used to selectively improve the quality of the measured signals. This concerns the rather broad spectra typically seen in solid samples, which mainly arise from static, direct dipolar couplings between nuclear spins, with other contributions due to chemical shielding and nuclear electric quadrupole interactions being of a lesser importance for the nuclei in our scope. One such technique, referred to as Magic Angle Spinning (MAS), is probably the most famous and popular, 
and is based on the rotation of the sample along an axis which has an angle of $54.7^{\circ}$ (magic angle) to the static magnetic field axis. The result of such a setup is to average out a large part of the direct dipolar couplings for spin $-1 / 2$ nuclei (e.g. ${ }^{29} \mathrm{Si}$ ), and thus the measured signal is only dependent on the Zeeman interaction and the nuclei neighbours, and are free from the broad band features which would show up otherwise. This is a consequence of the presence of the dipolar-coupling, chemical shielding, and nuclear quadrupolar terms of the spin Hamiltonian of factors containing the form $3 \cos ^{2} \theta-1$, where $\theta$ stands for the angle between the internuclear distance vector and the external magnetic field direction. For macroscopic sample spinning rates which are fast enough, the rotational average $3 \cos ^{2} \theta-1$ for such a magic angle cancels out all the anisotropic contributions to the total spin Hamiltonian. The net effect results in gaining enough resolution so that signals from individual nuclei may be observed. In simple glasses, this allows us to quantify the different $\mathrm{Q}_{n}$ species. Currently, almost every solid state NMR measurement is done using such a technique. A second method that could be considered brute force aims at reducing the quadrupolar interactions of I $>1 / 2$ nuclei by increasing the static magnetic field. This is particularly useful for ${ }^{11} \mathrm{~B}$ to split the $\mathrm{BO}_{3}$ and $\mathrm{BO}_{4}$ frequency peaks and quantify the two populations. In the case of our study, $18.8 \mathrm{~T}$ measurements were done for that purpose.

\subsubsection{Pristine glasses short-range structure}

The NMR measurements were carried out at the Unité de Catalyse et de Chimie du Solide (Lille, France) through the TGIR-RMN framework. NMR signals were measured for ${ }^{11} \mathrm{~B}$, ${ }^{29} \mathrm{Si},{ }^{23} \mathrm{Na}$, and ${ }^{27} \mathrm{Al}$ nuclei. $400 \mathrm{MHz}$ measurements were made for all the nuclei with a Bruker AVANCE II machine, which operates by employing a magnetic field of $9.4 \mathrm{~T}$. In addition, to reach a better signal quality, an $800 \mathrm{MHz}$ Bruker AVANCE III machine using a $18.8 \mathrm{~T}$ magnetic field was employed to probe sodium, aluminium, and boron signals. The use of a higher magnetic field is particularly useful for boron, as it attenuates the quadrupole moment of the nuclei and, as a consequence, sharpens the peaks and allows their discrimination.

\section{Boron - 11}

The NMR measurements on ${ }^{11} \mathrm{~B}$ constitute a powerful tool to quantify the speciation of boron atoms within a glass. Thanks to pioneering studies of Bray and O'Keefe [25], Yun and Bray [119], and Dell et al. [33], it is possible to calculate the amount of three- and four-fold coordinated atoms by integration of the intensity of the two peaks above and below a chemical shift of $5 \mathrm{ppm}$, respectively. The addition of network modifiers transforms $\mathrm{BO}_{3}$ triangles ( $N 3$ amount) into $\mathrm{BO}_{4}$ tetrahedra ( $N_{4}$ amount), since they provide the additional bridging oxygen atom and also a charge compensation mechanism. Also, because of their respective oxidation states, the addition of an alkali will affect a single boron site, whereas two $\mathrm{BO}_{4}$ units would be affected in the case of an alkaline-earth.

This technique allows us to estimate the proportion of $\mathrm{BO}_{3}$ and $\mathrm{BO}_{4}$, units provided that the chemical composition is known with enough details. The latter is given for the different materials studied in Table 4.3. The N3/N4 proportions have been computed 


\begin{tabular}{c|cc}
\hline & ABS case & Real case \\
\hline$R$ & $\frac{\left[\mathrm{Na}_{2} \mathrm{O}+\mathrm{K}_{2} \mathrm{O}\right]}{\left[\mathrm{B}_{2} \mathrm{O}_{3}\right]}$ & $\frac{\left[\mathrm{Na}_{2} \mathrm{O}+\mathrm{K}_{2} \mathrm{O}+\mathrm{CaO}+\mathrm{BaO}-\mathrm{MgO}-\mathrm{Al}_{2} \mathrm{O}_{3}-\mathrm{ZnO}\right]}{\left[\mathrm{B}_{2} \mathrm{O}_{3}\right]}$ \\
\hline $\mathrm{K}$ & $\frac{\left[\mathrm{SiO}_{2}\right]}{\left[\mathrm{B}_{2} \mathrm{O}_{3}\right]}$ \\
$\mathrm{R}_{\max }$ & $0.5+\frac{\mathrm{K}}{16}$ \\
\hline $\mathrm{N} 4$ for $\mathrm{R} \leqslant \mathrm{R}_{\max }$ & $\mathrm{R}$ \\
for $\mathrm{R} \geqslant \mathrm{R}_{\max }$ & $\mathrm{R}_{\max }-\frac{0.25}{1+\mathrm{K}} \cdot\left(\mathrm{R}-\mathrm{R}_{\max }\right)$ \\
\hline
\end{tabular}

Table 4.2: $\mathrm{BO}_{4}$ calculation rules for alkali-borosilicate systems extracted from Yun and Bray [119], ABS case, and adapted for the complex systems we are concerned with, real case.

using the Dell and Bray [33 model, assuming two distinct situations which either consider the existence of alkali, boron, and silicon species (ABS case) or took into account every oxide that could generate $\mathrm{BO}_{4}$ or incorporate network modifiers, a situation which bears more resemblance to the real case. Table 4.2 shows the calculation rules used to estimate the $\mathrm{BO}_{3} / \mathrm{BO}_{4}$ population proportions. It should be mentioned that for the four materials, we are in the case where $K=\frac{\left[\mathrm{SiO}_{2}\right]}{\mathrm{B}_{2} \mathrm{O}_{3}}<8$.

From the formula listed in Table 4.2, the N4 proportions for the glasses studied here are estimated. The results are reported at the end of Table 4.3. The analysis of these values show that, for N-BK7 and S-BSL7, the excess of network modifiers should moderate the intermediate ions and the real N4 values should be of the same order. For Borofloat, one might think that, as its composition is quite simple and the behaviour of alumina in sodium borosilicate is well understood [39], the $\mathrm{BO}_{4}$ content should be similar to the predicted value. Finally, the negative "N4 (real)" value for N-ZK7 tells us that the Dell and Bray model cannot be adopted for that glass since the content of intermediate ions is higher than that characteristic of network modifiers. In addition, the phase segregation observed by TEM imaging gives further support to the view that shows N-ZK7 as far different from a ternary system.

$\mathrm{BO}_{3}$ and $\mathbf{B O}_{4}$ sites: From inspection of the ${ }^{11} \mathrm{~B}$ MAS-NMR spectra (see Fig. 4.8a), one notices two main peaks more or less symmetrically displaced from $5 \mathrm{ppm}$. The peak spanning from 5 to $20 \mathrm{ppm}$ is attributed to $\mathrm{BO}_{3}$ triangles, while the other corresponds to $\mathrm{BO}_{4}$ tetrahedra. As explained earlier, the integration of the peaks and their ratio gives the N4/N3 proportion. This calculation has been performed using the DMfit software 71 . Integration results are reported in Table 4.4. One can see that the estimated values for Borofloat are in very good agreement with those calculated from the NMR curve. This confirms the fact that in alumino-borosilicate glasses, the alkali atoms are preferentially bonded to aluminium to convert them into network formers with four-fold coordination. For N-BK7 and S-BSL7, the direct derivation of N4 is even higher than predicted from the chemical composition. This will be tempered in the next paragraph when analysing the spectra more finely. N-ZK7 has about one half of the "ABS case" N4 amount. Further interpretations using ${ }^{11} \mathrm{~B}$ MAS-NMR results concerning the zinc and 


\begin{tabular}{c|cccc}
\hline mol. \% & Borofloat & N-ZK7 & N-BK7 & S-BSL7 \\
\hline $\mathrm{SiO}_{2}$ & 82 & 66 & 73 & 73.5 \\
$\mathrm{~B}_{2} \mathrm{O}_{3}$ & 12 & 11.5 & 10 & 9.5 \\
\hline $\mathrm{Na}_{2} \mathrm{O}$ & 4 & 7.5 & 10 & 10 \\
$\mathrm{~K}_{2} \mathrm{O}$ & 0.5 & - & 5 & 5.5 \\
$\mathrm{CaO}$ & - & 1 & 0.25 & - \\
$\mathrm{BaO}$ & - & - & 0.25 & 0.5 \\
\hline $\mathrm{MgO}$ & - & - & 1.25 & - \\
$\mathrm{Al}_{2} \mathrm{O}_{3}$ & 1.5 & 4 & 0.25 & - \\
$\mathrm{ZnO}$ & - & 10 & - & 1 \\
\hline \hline \multicolumn{5}{c}{ Estimated values } \\
$\mathrm{N} 4$ ABS (\%) & 36 & 63 & 95 & 97 \\
$\mathrm{~N} 4$ real $(\%)$ & 22 & -48 & 95 & 97 \\
\hline
\end{tabular}

Table 4.3: Chemical composition and corresponding estimated N4 proportions

aluminium roles are difficult to support.

It is worth refining the analysis of $\mathrm{BO}_{4}$ peak for N-BK7 and S-BSL7 specifically. In addition to the spin Central Transition (CT) $(1 / 2 \mid-1 / 2)$, boron can undergo transitions such as $(3 / 2 \mid 1 / 2)$ and $(-1 / 2 \mid-3 / 2)$. These are called Satellite Transitions (ST), and contribute to the CT signal used previously for quantification. ST can be detected as rotation bands; see the peaks located around $-80 \mathrm{ppm}$ in Fig. 4.8b. As it is not possible to discriminate the isotropic STs hidden in the $\mathrm{CT}$, their intensity is approximated to that of the first rotation bands. Then, to derive the $\mathrm{BO}_{3} / \mathrm{BO}_{4}$ proportions, the intensity from the STs are subtracted from the CT. This calculation results in a modification of the boron coordination proportion (see the last two lines of Table 4.4). Thus one can see that the corrected values do not deviate much for Borofloat and N-ZK7 due to the fact that $\mathrm{ST}$ signal is strong for $\mathrm{BO}_{4}$ units. For the two other glasses, as their $\mathrm{BO}_{4}$ population was higher, the coordination proportion is more affected. Due to the difficulty of quantifying the isotropic ST intensity, the error on $\mathrm{BO}_{3} / \mathrm{BO}_{4}$ proportions are of the order of some percent. These new and consolidated values prove that these glasses do not behave like the simple ternary systems as described by Yun and Bray.

$\mathrm{BO}_{4}$ speciation: As can be seen from Fig. 4.8a, particularly for Borofloat, the $\mathrm{BO}_{4}$ peak shows two individual contributors around $0 \mathrm{ppm}$ and $-2 \mathrm{ppm}$, respectively. They have been attributed to four-fold boron nuclei linked to three and four silicon atoms, respectively [10,11] . These are often written $\mathrm{BO}_{4}(1 \mathrm{~B}, 3 \mathrm{Si})$ and $\mathrm{BO}_{4}(0 \mathrm{~B}, 4 \mathrm{Si})$. Such an assignment relies in the fact that danburite and reedmergnerite crystals have their MASNMR peaks with chemical shifts $0.7 \mathrm{ppm}$ and $-1.9 \mathrm{ppm}$, respectively. The software DMfit [71] has been used to fit the $\mathrm{BO}_{4}$ peaks of the NMR spectra. Two contributions corresponding to the units mentioned earlier were used to fit the curve. The graphical results of these fits can be seen for N-BK7 in Fig. 4.9a and 4.9b, and the calculated species 


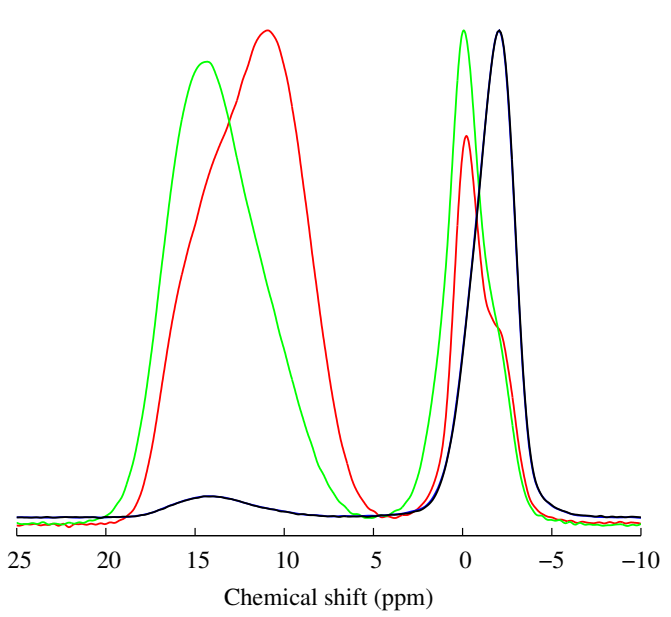

(a) ${ }^{11}$ Boron spectra

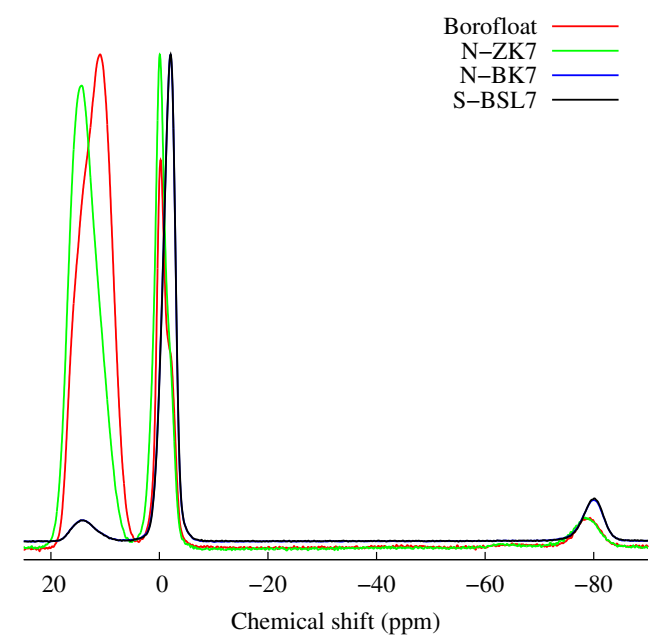

(b) Central and Satellite Transition

Figure 4.8: ${ }^{11}$ Boron MAS-NMR signal from the four glasses, $\mathrm{B}_{0}=18.8 \mathrm{~T}$.

\begin{tabular}{|c|c|c|c|c|}
\hline$\%$ & Borofloat & $\mathrm{N}-\mathrm{ZK} 7$ & N-BK7 & S-BSL7 \\
\hline \multicolumn{5}{|c|}{ Direct calculation } \\
\hline N3 & 78 & 68 & 2.25 & 2 \\
\hline N4 & 22 & 32 & 97.75 & 98 \\
\hline \multicolumn{5}{|c|}{$\mathrm{BO}_{4}$ Satellite Transitions subtracted } \\
\hline N3 & 77 & 70 & 8.5 & 10 \\
\hline N4 & 23 & 30 & 91.5 & 90 \\
\hline
\end{tabular}

Table 4.4: N3 and N4 proportion for the pristine glasses. Calculated from $800 \mathrm{MHz}$ MAS-NMR ${ }^{11} \mathrm{~B}$ measurements with DMfit software. 


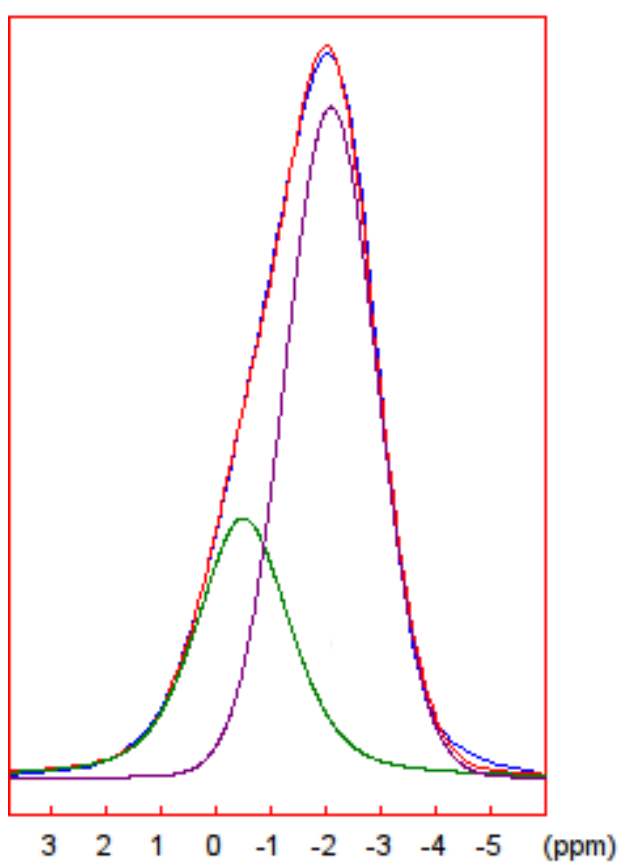

(a) Direct $\mathrm{BO}_{4}$ fitting

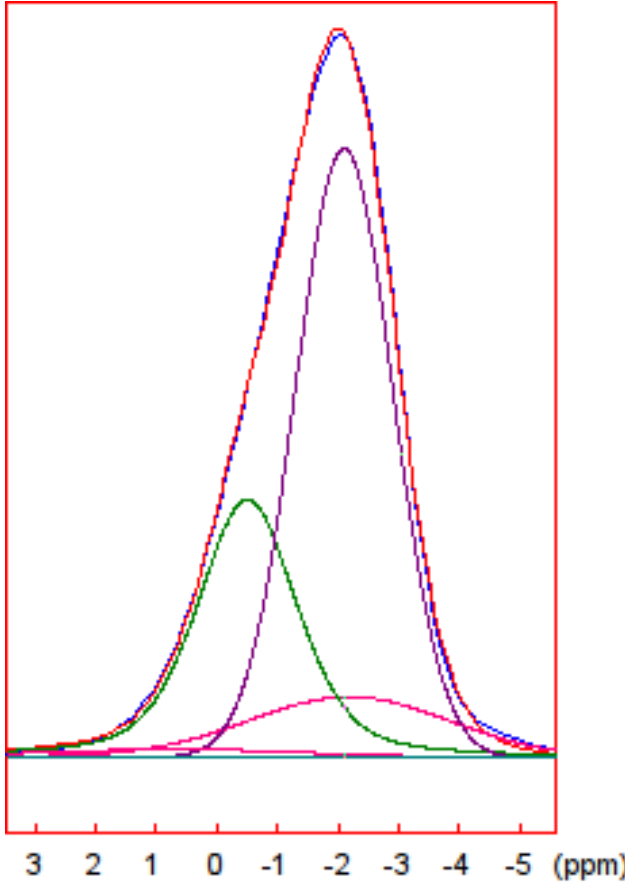

(b) Satellite transitions considered

Figure 4.9: Fitting of $\mathrm{BO}_{4}$ MAS-NMR peak (in blue) with two contributions corresponding to $\mathrm{BO}_{4}(1 \mathrm{~B}, 3 \mathrm{Si})$ and $\mathrm{BO}_{4}(0 \mathrm{~B}, 4 \mathrm{Si})$ in green and purple respectively. In Fig. $4.9 \mathrm{~b}$, the satellite transitions are in pink. The combination of the different contributions is in red. $\mathrm{B}_{0}=18.8 \mathrm{~T}$

distribution can be seen in Table 4.5. Calculations have been done in both cases, taking into account the Satellite Transitions. Looking at the results, we see that correcting the signal changes the proportions from around 4 to $12 \%$. Specifically, ST seems to mainly pollute the $\mathrm{BO}_{4}(0 \mathrm{~B}, 4 \mathrm{Si})$; see the $\mathrm{N}-\mathrm{BK} 7$ fitting as an example.

From those results, one can see that there are two different kinds of speciation. On the one hand, Borofloat and N-ZK7 have a majority of $\mathrm{BO}_{4}(1 \mathrm{~B}, 3 \mathrm{Si})$ units; in contrast, N-BK7 and S-BSL7 almost have a single type of boron units: $\mathrm{BO}_{4}(0 \mathrm{~B}, 4 \mathrm{Si})$. One should note that the integral values should be taken with care, as the fit is not perfect, especially on the edges of the peak. Considering the complexity of these glass compositions, one could think the presence of a third contribution was arising from the interaction of boron and zinc or aluminium. The current data, however, limits our ability to proceed beyond this point.

\section{Silicon -29}

Establishing the discrimination of the different silicon environments becomes a bit harder than in the case of boron. Up to a first order, there are contributions of the different $\mathrm{Q}_{\mathrm{n}}$ species, and for each case the nature of the second neighbour will also affect the signal. In other words, the final signal will be dependent upon whether they are only silicon or if there is $\mathrm{BO}_{3}, \mathrm{BO}_{4}$, or aluminium involved, which would lead to cases denoted by $\mathrm{Q}_{n}(\mathrm{~B} 3)$, $\mathrm{Q}_{n}(\mathrm{~B} 4)$, and $\mathrm{Q}_{n}(\mathrm{nAl})$, respectively. In the study from Lippmaa et al. [63], for each 


\begin{tabular}{lcccc}
\hline & \multicolumn{5}{c}{ Borofloat } & N-ZK7 & N-BK7, S-BSL7 \\
\hline \multicolumn{5}{c}{ Direct calculation } \\
\hline $\mathrm{BO}_{4}(1 \mathrm{~B}, 3 \mathrm{Si})$ & Position $\delta(\mathrm{ppm})$ & -0.19 & -0.1 & -0.5 \\
& Amount (\%) & 71 & 92 & 31 \\
\hline \multirow{2}{*}{$\mathrm{BO}_{4}(0 \mathrm{~B}, 4 \mathrm{Si})$} & Position $\delta(\mathrm{ppm})$ & -2.06 & -2.16 & -2.1 \\
& Amount $(\%)$ & 29 & 8 & 69 \\
\hline \multirow{5}{*}{$\mathrm{BO}_{4}(1 \mathrm{~B}, 3 \mathrm{Si})$} & Position $\delta(\mathrm{ppm})$ & -0.19 & -0.1 & -0.5 \\
& Amount (\%) & 68 & 96 & 35 \\
\hline \multirow{2}{*}{$\mathrm{BO}_{4}(0 \mathrm{~B}, 4 \mathrm{Si})$} & Position $\delta(\mathrm{ppm})$ & -2.04 & -2.17 & -2.1 \\
& Amount (\%) & 32 & 4 & 65 \\
\hline
\end{tabular}

Table 4.5: $\mathrm{BO}_{4}$ speciation from the MAS-NMR curve fitting of Fig. 4.9 ,

\begin{tabular}{|c|c|c|c|c|}
\hline & Borofloat & N-ZK7 & N-BK7 & S-BSL7 \\
\hline \multicolumn{5}{|c|}{ No zinc or manganese charge compensation } \\
\hline $\mathrm{NBO}^{\text {est. }}$ & 0.0029 & 0.016 & 0.08 & 0.10 \\
\hline \multicolumn{5}{|c|}{ Zinc or manganese charge compensated } \\
\hline $\mathrm{NBO}^{\text {est. }}$ & 0.0029 & -0.14 & 0.07 & 0.09 \\
\hline
\end{tabular}

Table 4.6: Proportion of non-bridging oxygen per silicon atom, estimated from the network formers remaining.

aluminium atom replacing a silicon in a $\mathrm{Q}_{4}$ unit, its chemical shift gains $5 \mathrm{ppm}$. Before considering the MAS-NMR curves, it is worth estimating the number of non-bridging oxygen per silicon atoms from the N4 values calculated in the previous pages. This can be carried out by means of the following expression,

$$
\mathrm{NBO}^{\text {est. }}=\frac{\left[\mathrm{Na}_{2} \mathrm{O}\right]+\left[\mathrm{K}_{2} \mathrm{O}\right]+[\mathrm{CaO}]+[\mathrm{BaO}]-\mathrm{N} 4 \cdot\left[\mathrm{B}_{2} \mathrm{O}_{3}\right]-\mathrm{Al}_{2} \mathrm{O}_{3}-(\mathrm{ZnO}+\mathrm{MgO})}{\left[\mathrm{SiO}_{2}\right]},
$$

which takes into account the network modifiers not used by the boron atoms from where one has to subtract the alumina content. An extreme case can be figured out by considering that $\mathrm{MgO}$ and $\mathrm{ZnO}$ will be preferentially charge compensated. Table 4.6 compiles the results of these calculations. Once again, the value for N-ZK7 in the case of a full charge compensation of zinc oxide confirms the complexity of this glass. As observed from the STEM atom mapping, aluminium and zinc rich zones also concentrate sodium. However, considering the different contents, one can be sure that zinc is not fully within four-fold coordination.

Figure 4.10 compiles the MAS-NMR signals from ${ }^{29} \mathrm{Si}$ for the four materials. Starting with Borofloat, the peak is almost a perfect Gaussian that could be assigned to $\mathrm{Q}_{4}$ alone, with only silicon as a second neighbour. In addition, the peak centred around $-110 \mathrm{ppm}$ can be compared to that of quartz, which appears at $-107.4 \mathrm{ppm}$ 63. A close inspection 


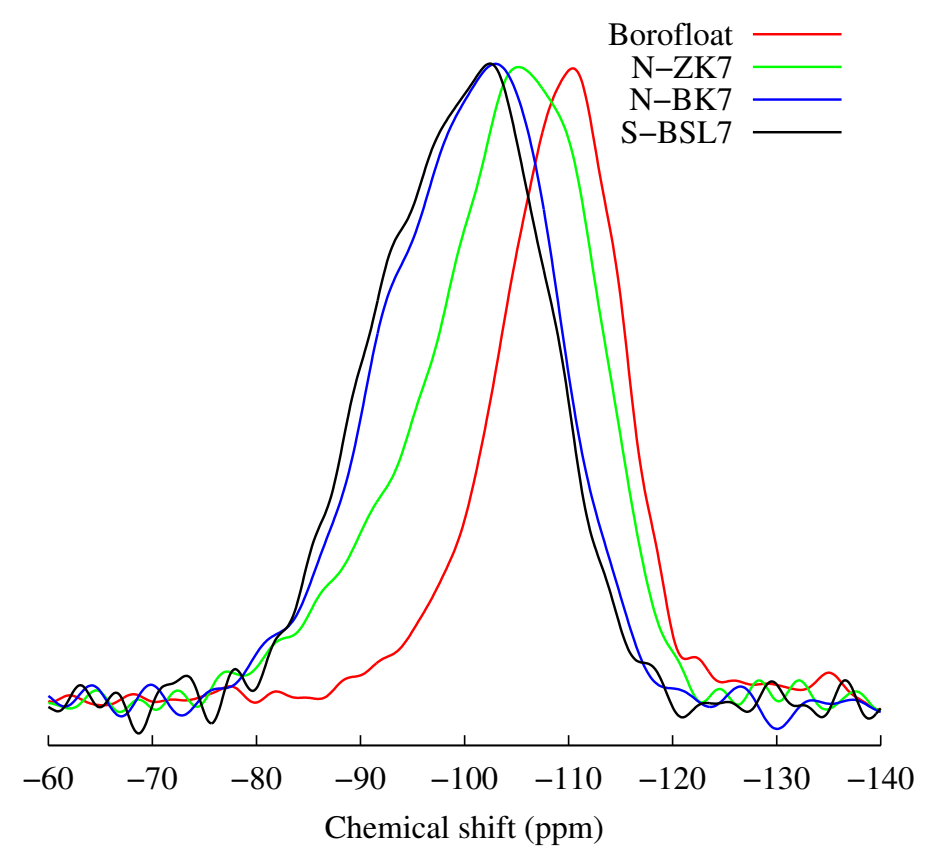

Figure 4.10: ${ }^{29}$ Silicon MAS-NMR spectra, $\mathrm{B}_{0}=9.4 \mathrm{~T}$.

at the lineshape shows a significant asymmetry evidenced by a sharper right edge. Such an effect unveils a small contribution coming from $\mathrm{Q}_{4}$ linked with $\mathrm{Al}$ or $\mathrm{B}$. The peak for N-ZK7, which has a mean shift around $-105 \mathrm{ppm}$, is also compatible with a high predominance of $\mathrm{Q}_{4}$. The broadening of the curve is difficult to interpret, as this material has a high concentration of zinc that may also affect the NMR signal. The fact that the maximum NBO per Si is only $2.48 \%$ for this glass confirms that most of the silica network must be composed of $\mathrm{Q}_{4}$ units. The last two curves from N-BK7 and S-BSL7 are broader and centered at a significantly higher chemical shift compared to the other glasses. One can notice that half of the curve is located above $-100 \mathrm{ppm}$ which is the signature of at least some $Q_{3}$ units. In fact, the study from Shneider et al. 102 shows their contribution to be located around -94 to $-88 \mathrm{ppm}$, depending upon the network modifier. Finally, compared to N-BK7, S-BSL7 is located at a slightly higher frequency, which can be attributed to a higher content in $\mathrm{Q}_{3}$ and perhaps $\mathrm{Q}_{2}$. This fact is confirmed by the higher $\mathrm{NBO}^{\text {est. }}$ for that glass.

\section{Aluminium -27 and sodium -23}

The coordination of aluminium can be extracted from its MAS-NMR spectra. As it can be seen from Fig. 4.11a, for Borofloat, and N-ZK7, the peak is located around 55 ppm which is assigned to four-fold coordinated atoms. The main significance of the current results stems from unequivocal proof showing that alkali and alkaline-earth are preferentially linked to aluminium to turn it into a network former.

Because of its nature as a network modifier, the interpretation of sodium-23 MAS-NMR spectra does not provide further additional information on the glass structure. Looking at Fig. 4.11b, it can be seen that the shape of the peak is similar for the four glasses, but somewhat drifts towards higher shifts in apparent correlation with the sodium content. 


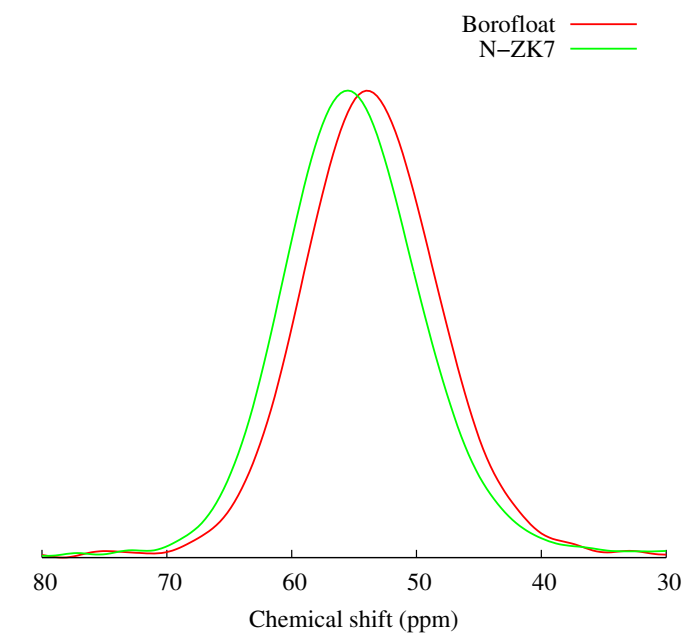

(a) ${ }^{27}$ Aluminium MAS-NMR spectra, $\mathrm{B}_{0}=18.8 \mathrm{~T}$.

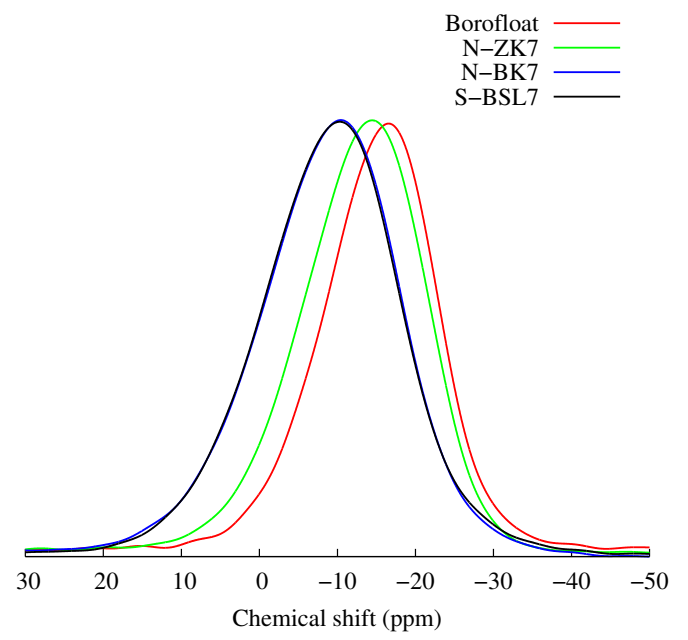

(b) ${ }^{23}$ Sodium MAS-NMR spectra, $\mathrm{B}_{0}=18.8 \mathrm{~T}$.

Such a displacement of the sodium peak has been related to its structural role in the glass network [12]. This chemical element would act more as a network modifier than a charge compensator in the case of S-BSL7 and N-BK7 than for the two other glasses, which is in agreement with findings shown by the ${ }^{29} \mathrm{Si}$ spectrum. This is coherent; for Borofloat and $\mathrm{N}-Z K 7, \mathrm{Na}$ is mainly used as charge compensator, while N-BK7 and S-BSL7 utilize most of the boron in four-fold coordination, and the excess of alkali is used to create NBOs.

\subsubsection{Evidence of changes in short range structure of irradiated glasses}

To ascertain possible changes in the short range structure due to irradiation, NMR experiments were carried out at the Institute for Transuranium Elements (ITU, Karlsruhe, Germany) using a $400 \mathrm{MHz}$ spectrometer. This was the only instrument we were able to find to carry out measurements on radiation activated samples. Because of the high demand of this equipment, only two samples could be measured. Both Borofloat and S-BSL7 were chosen for the purpose and in particular, the selected samples were those which received the neutron dose F3 from Table 3.8. The rationale behind this selection was based on the fact that these two glasses have very different vitreous structure. In addition, their behaviour under irradiation shows Borofloat as the glass showing more marked changes than N-ZK7 under neutron flux while having less radioactivity. Also, the choice of the samples which received the F3 dose was made on the grounds that it constitutes the best compromise between macroscopic variations and sample radioactivity. As regards the reasons for selecting S-BSL7, this was made since, contrary to N-BK7, Raman data were available for such a sample.

The measurements described here were performed under an applied magnetic field of $9.4 \mathrm{~T}$. They are compared to their pristine glass curve recorded at the same field, but not shown earlier as they are less accurate. The reason behind this is that signals recorded at different magnetic fields are difficult to compare because, as explained earlier for ${ }^{11} \mathrm{~B}$, 


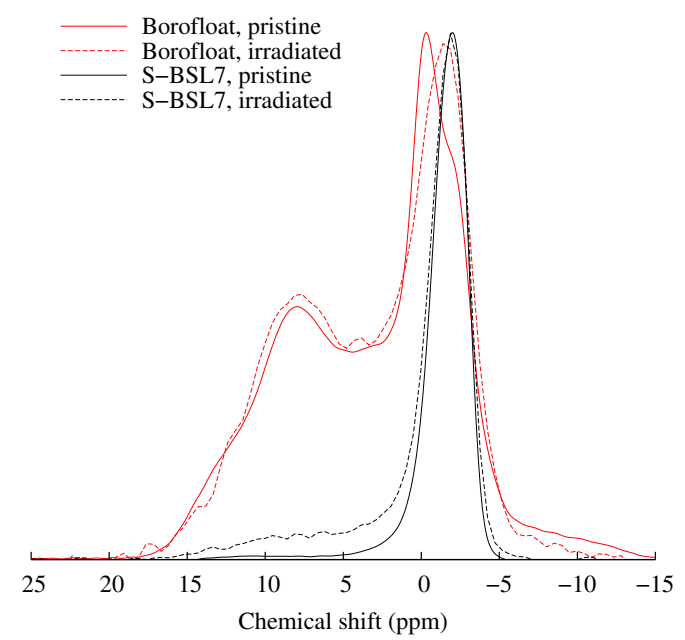

(a) Global view

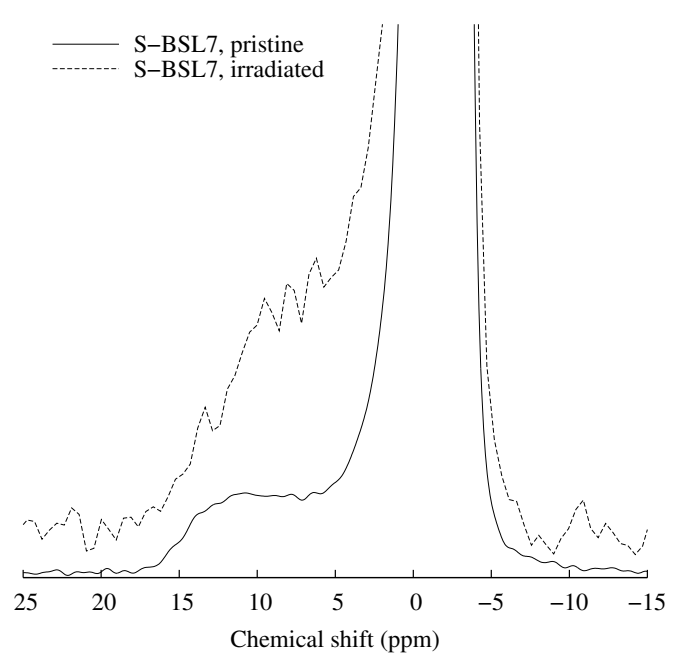

(b) Zoom on $\mathrm{BO}_{3}$ band of S-BSL7

Figure 4.12: Borofloat and S-BSL7 MAS-NMR ${ }^{11} \mathrm{~B}$ spectra, pristine and irradiated state.

${ }^{23} \mathrm{Na}$, and ${ }^{27} \mathrm{Al}$ nuclei, the field intensity strongly reduces the quadrupolar interaction.

\section{Boron - 11}

As mentioned above, irradiated Borofloat and S-BSL7 were selected because the features of their NMR spectra for pristine samples reveal a spectrum for S-BSL7 for this nucleus dominated by $\mathrm{BO}_{4}$ sites, whereas the spectrum for Borofloat shows a lineshape resulting from a combination of three- and four-fold coordinated atoms. The latter clearly shows a $\mathrm{BO}_{4}$ peak, revealing the existence of two populations with different co-ordination, namely $\mathrm{BO}_{4}(3 \mathrm{Si}, 1 \mathrm{~B})$ and $\mathrm{BO}_{4}(4 \mathrm{Si}, 0 \mathrm{~B})$. Spectra for both pristine and irradiated spectra from S-BSL7 and Borofloat are gathered in Fig. 4.12a. Notice that the signal coming from the irradiated samples is noisier than those from the pristine state, which is a result of the smaller amount of material that could be used for such measurements.

The interpretation of spectra for Borofloat in terms of boron coordination is a bit difficult due to the relatively high content of $\mathrm{BO}_{3}$ units that contribute to the MASNMR intensity within a region of chemical shifts located from $-15 \mathrm{ppm}$ to $20 \mathrm{ppm}$. As a first result, we see from the referred figure that the most intense peak is shifted to the right. The shoulder around $-2 \mathrm{ppm}$ in the pristine sample, signing the existence of a small amount of $\mathrm{BO}_{4}(4 \mathrm{Si}, 0 \mathrm{~B})$, is no longer discernible after irradiation. This suggests a conversion of $\mathrm{BO}_{4}(3 \mathrm{Si}, 1 \mathrm{~B})$ into $\mathrm{BO}_{4}(4 \mathrm{Si}, 0 \mathrm{~B})$ as the dominant radiation effect as far as the local environment of boron nuclei are concerned. A line profile analysis was then carried out, and the resulting fits are shown in Fig 4.13 a-b. Such an exercise was performed using two quadrupolar MAS spin one half contributions to simulate $\mathrm{BO}_{3}$ signal, and two Gaussian lines for $\mathrm{BO}_{4}$. The parameters were optimized in order to find boron speciation values consistent with those obtained within the $800 \mathrm{MHz}$ experiments. The results of the data treatment show a rather slight growth of the $\mathrm{BO}_{3}$ population, from $77 \%$ to $79 \%$, and a noticeable increase of $\mathrm{BO}_{4}(4 \mathrm{Si}, 0 \mathrm{~B})$ from $32 \%$ to $49 \%$. The former result is to be taken with care, considering the uncertainties in both data and fitting procedure itself. 


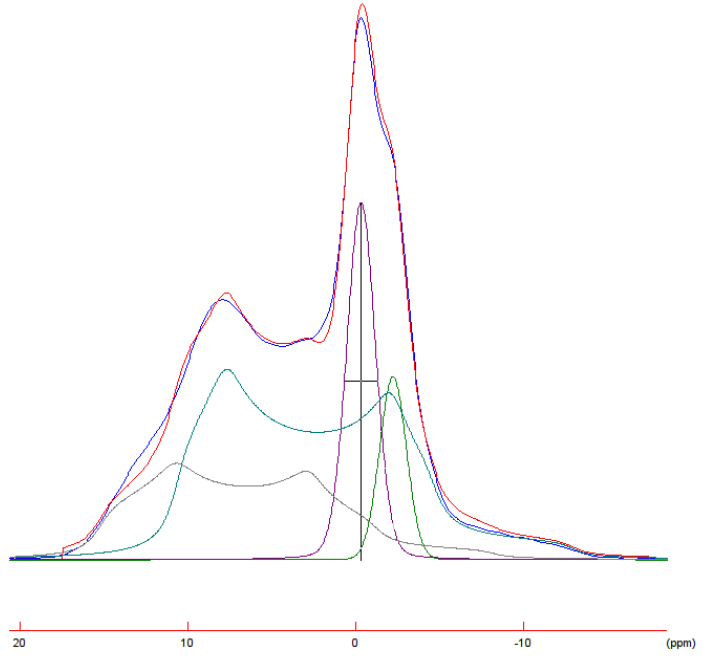

(a) Pristine

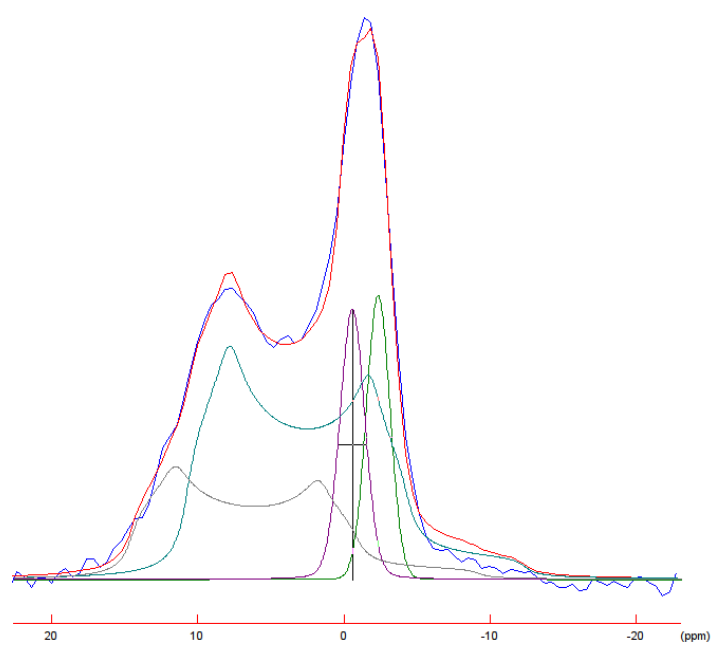

(b) Irradiated

Figure 4.13: Borofloat 11B MAS-NMR spectra and fitted curves with 4 contributions, $\mathrm{B}_{0}=9.4 \mathrm{~T}$.

However, the magnitude of the conversion among $\mathrm{BO}_{4}$ population is large enough to infer that thermal neutron irradiation favours the existence of $\mathrm{BO}_{4}(4 \mathrm{Si}, 0 \mathrm{~B})$. This could be explained by the fact that $(\mathrm{n}, \alpha)$ reactions, through ballistic mixing, break up $\mathrm{BO}_{4}-\mathrm{BO}_{4}$ pairs and distribute them homogeneously within the silica network.

The spectrum for S-BSL7 shows a noticeable increase in the $\mathrm{BO}_{3}$ population, as witnessed by the increase in intensity of the broad feature centred about $7.5 \mathrm{ppm}$, see Fig. 4.12b. Considering the speciation of $\mathrm{BO}_{4}$ units, irradiation does not seem to involve drastic conversions from one coordination into the other. The peak remains centred around $-2 \mathrm{ppm}$. A line profile analysis was then carried out, and the resulting fits are shown in Fig. 4.14 a-b. Considering the high amount of $\mathrm{BO}_{4}$ units and the fact that its population is modified by irradiation, special care was taken in order to deal with the contribution in the line intensity arising from the satellite transitions contained in the CT. The results from line profile fitting shows that the $\mathrm{BO}_{3}$ population increases from some $12 \%$ up to $22 \%$. Some comments on such results are pertinent here. First, the figure for the population for the pristine sample is in good agreement with that previously derived from the high-field, $18.8 \mathrm{~T}$ measurements. Second, our result shows that irradiation induces a modification of the boron coordination, in some ways analogous to findings reported by Peuget et al. [83] on borosilicate glass irradiated with Xenon ions. Such conversion of boron tetrahedra into triangles is followed by a release of network modifiers within the vitreous network, which would lead to an increase in the population of non-bridging oxygen.

The results just discussed show that boron environments are modified by neutron radiation in rather different ways for the two glasses. For Borofloat, which is rich in $\mathrm{BO}_{3}$ units and has a $\mathrm{BO}_{4}$ population dominated by $(3 \mathrm{Si}, 1 \mathrm{~B})$ species, its three-fold coordinated population is not largely affected by radiation, whereas its $\mathrm{BO}_{4}$ population gets more homogenized through the conversion of $\mathrm{BO}_{4}(3 \mathrm{Si}, 1 \mathrm{~B})$ into $\mathrm{BO}_{4}(4 \mathrm{Si}, 0 \mathrm{~B})$. On the other hand, S-BSL7, which is rich in $\mathrm{BO}_{4}$ dominated by (4Si, 0B) species, generates three-fold 


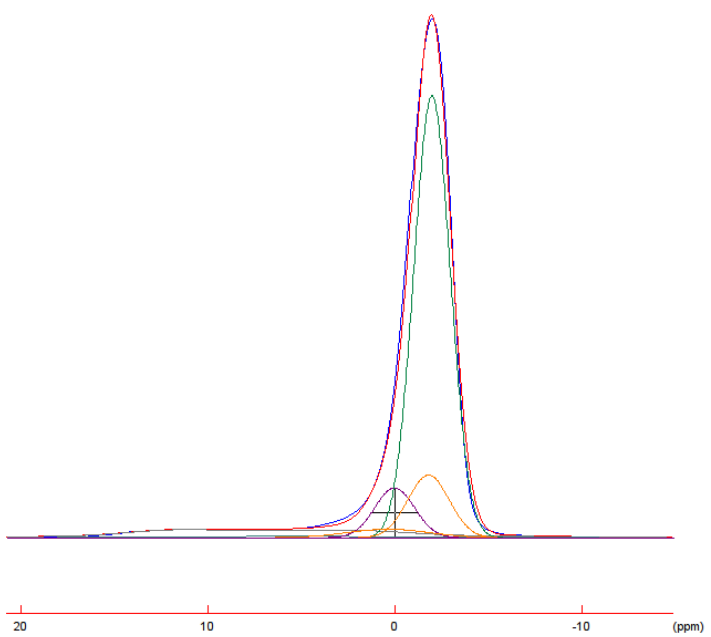

(a) Pristine

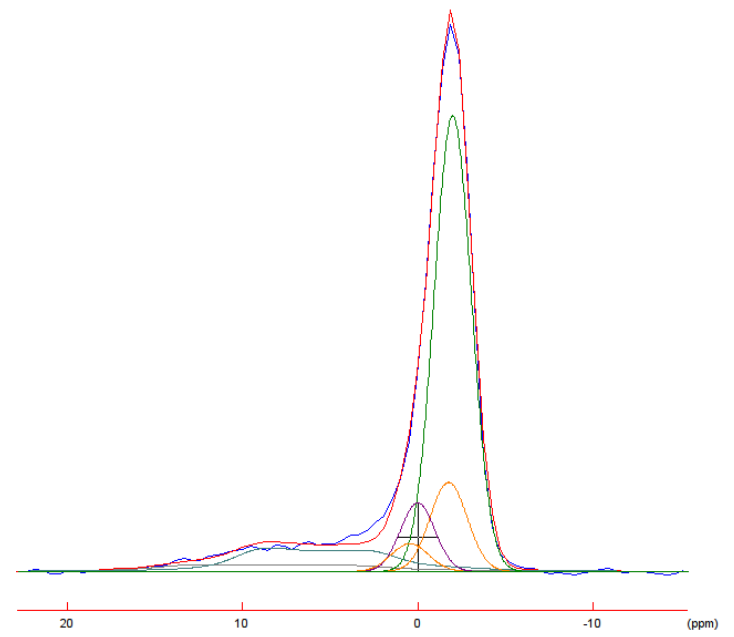

(b) Irradiated

Figure 4.14: S-BSL7 11B MAS-NMR spectra and fitted curves with 4 contributions, $\mathrm{B}_{0}=9.4 \mathrm{~T}$.

coordinated boron atoms, while its $\mathrm{BO}_{4}$ speciation is not affected and stays dominated by boron atoms surrounded by four silicons.

Sodium -23

The ${ }^{23} \mathrm{Na}$ MAS-NMR spectra of pristine and irradiated glasses are reported in Fig 4.15 . The dominant peak in the spectrum for S-BSL7 significantly broadens towards the region of lower chemical shifts. The observed changes for Borofloat are significantly less marked, with a small broadening discernible in the trailing edge of the spectrum. Notice however that in both cases the maxima of the asymmetric peak is not significantly changed. In their study, Peuget et al. [83] observed both a broadening and a shift of the peak toward higher values. The measurements reported here could then suggest that only a slight modification of the sodium ions takes place, which either become more network modifiers or charge compensators for S-BSL7 and Borofloat, respectively.

\subsection{Neutron scattering}

\subsubsection{Introduction}

To obtain information regarding structural arrangements at the microscopic scale, we make use of the wave-like properties of a thermal neutron beam. Although the de Broglie wavelength of a monochromatic neutron beam, $\lambda$, is a scalar property, the neutron wave has a well defined direction along that followed by the neutron velocity vector. To specify such a direction, as well as the associated vector modulus, we make recourse of a vectorial quantity such as the neutron wavevector,

$$
\mathbf{k}=\frac{m_{n} \mathbf{v}}{\hbar}
$$




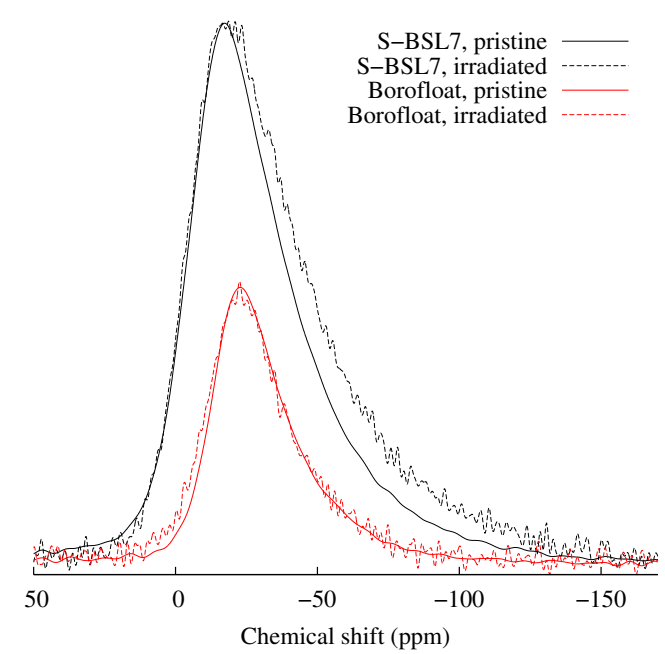

Figure 4.15: ${ }^{23}$ Sodium MAS-NMR spectra, $\mathrm{B}_{0}=9.4 \mathrm{~T}$.

where $m_{n}$ stands for the neutron rest mass and $\mathbf{v}$ is the velocity vector. To give some numerics, say that the vector modulus, given in terms of the scalar velocity, yields $k\left(n m^{-1}\right)=15.882 \times v(\mathrm{~mm} / \mu \mathrm{s})$.

To study the structure of a given condensed body, we determine the scattered neutron intensity as a function of the incident $\mathbf{k}_{\mathbf{0}}$, scattered wavevector $\mathbf{k}_{\mathbf{f}}$, and the scattering angle $\theta$. In more detail, consider the scattering of an incident plane wave from a single scattering center located at position $\mathbf{r}^{\prime}$. The quantity of interest will be the ratio of scattered to incident neutron fluxes and, for such an avail, assume an incident beam with unit density expressed by the wave equation,

$$
\Psi_{0}=e^{i \mathbf{k}_{\mathbf{0}} \cdot \mathbf{r}}
$$

which fully characterizes the incident flux; in turn, this is defined as the number of neutrons crossing a surface of unit area per unit time, and therefore its flux equals $\hbar k_{0} / m_{n}$. The scattered wave from our point scatterer will now be a spherical wave centred on $\mathbf{r}^{\prime}$, which will have some scattering amplitude $A$,

$$
\Psi_{f}=A \frac{e^{i \mathbf{k}_{f} \cdot\left(\mathbf{r}-\mathbf{r}^{\prime}\right)}}{\left|r-r^{\prime}\right|}=A \frac{e^{i \mathbf{k}_{f} \cdot \mathbf{R}}}{|\mathbf{R}|},
$$

where $\mathbf{R}=\mathbf{r}-\mathbf{r}^{\prime}$. The density of such a wave which propagates with a velocity $\hbar k_{i} / m_{n}$ amounts to $|A|^{2} / R^{2}$, and consequently we can define the neutron flux crossing an area $R^{2} d \Omega$ by

$$
\frac{|A|^{2}}{R^{2}} \frac{\hbar k_{f}}{m_{n}} R^{2} d \Omega
$$

from where the ratio of scattered to incident neutron fluxes yields,

$$
\frac{\mathrm{d} \sigma}{\mathrm{d} \Omega}=\frac{k_{f}}{k_{0}}\left|A^{2}\right|
$$

a quantity known as the differential cross-section. Since the scattering amplitude has 
dimensions of length, the cross-section will have dimensions of surface.

A single nucleus within a neutron beam behaves just like a point scatterer. The force felt by the incoming neutron is short-ranged, of the order of $10^{-15} \mathrm{~m}$, that is far shorter than the wavelengths of neutrons employed for Condensed Matter, studies which typically are within the tenths of a nanometer up to a few tens of nanometer range. The scattering amplitude $\left|A^{2}\right|$ for an isolated nucleus at rest is thus independent of the scattering angle, and is usually denoted as $-b$ and named the scattering length which for most nuclei has positive values of a few times $10^{-15} \mathrm{~m}$. The scattering lengths usually show significant variations for the different isotopes of a given element, and in case that the nucleus has a non-zero spin, the different spin states also show different scattering lengths. This means that, unless specific isotopic enrichment techniques are used, the scattering length of an element at its natural abundance will be an average or a mean scattering length. A second contribution arises from deviations of the average scattering length from its mean value, which arise from the different contributions of the different $2 I+1$ spin states that may exist for nuclei with spin higher than one half.

Let us consider the scattering from an ensemble of $N$ nuclei at positions $\mathbf{R}_{\mathbf{i}}$. Notice that nuclei located at $\mathbf{R}_{\mathbf{i}}$ will induce a phase-shift in the scattered wave, given by the differences in phases between the incident and scattered beam. The net effect of this is if the the cross-section is left unchanged, the amplitude now includes such a phase shift. The ensemble of nuclei under consideration will yield a total wave composed by waves scattered from individual nuclei which, because of the phase shift just referred to, will interfere and yield a total amplitude

$$
A=\sum_{i}^{N}-b_{N} e^{i\left(\mathbf{k}-\mathbf{k}_{\mathbf{f}}\right) \cdot \mathbf{R}},
$$

which yields a cross-section,

$$
\frac{d \sigma}{d \Omega}=\left|\sum_{i}^{N} b_{N} e^{i\left(\mathbf{k}_{\mathbf{0}}-\mathbf{k}_{\mathbf{f}}\right) \cdot \mathbf{R}}\right|^{2} .
$$

From the equation given above, we see that the cross-section is dependent upon the difference in wavevectors $\mathbf{k}_{\mathbf{0}}-\mathbf{k}_{\mathbf{f}}$, a quantity usually referred to as the scattering vector

$$
Q=2 k_{0} \sin \theta=\frac{4 \pi \sin (\theta)}{\lambda}
$$

For perfect crystals, elastic scattering occurs when the Bragg resonance condition is fulfilled, that is

$$
n \lambda=2 d \sin \theta
$$

where $d$ stands for the lattice spacing, and clearly the minimum possible momentum transfer corresponds to $n=1$ with $q=2 \pi / d$. Real crystals, however, may contain defects and heterogeneities having dimensions significantly larger than $d$ such as lattice defects, dislocations, and voids, which can be studied within the regime called "small angle scattering"; that is, $0<q<\pi / d$. It goes without saying that the technique can also be applied to the study of non-crystalline materials. In fact, the technique has been successfully applied for decades to the study of a wide variety of systems, such as 
polymers and biological macromolecules, precipitates in solution, colloidal systems, etc. However, to the best of our knowledge, it has not been applied to the study of nanoscale heterogeneities in glass samples, which is the main focus of our interest.

\subsubsection{Scattering from large-scale structures : General principles}

The technique known as neutron small angle scattering is now a well established tool for the detection of sample heterogeneities having a typical size between $10 \mathrm{~nm}$ and $100 \mathrm{~nm}$ [111]. The measurements are carried out at scattering angles down to a thousandth of a radian using cold neutrons. Under such conditions, interference between individual atoms becomes unimportant, and the quantity to consider is the fluctuation in the neutron scattering length density $b(r)$. Such a quantity is usually defined by recourse to the specification of some average "molecular unit" of volume $V_{\text {res }}$, so that

$$
b(r)=\sum_{i} b_{i} / V_{\text {res }}
$$

where $b_{i}$ is the individual coherent scattering amplitude and $V_{\text {res }}$ is the elementary volume.

The differential cross-section is calculated as an integral over the sample volume $V_{s}$ and given in terms of differences with respect to the mean scattering length $\bar{b}$ and yields 113,

$$
\frac{d \sigma}{d \Omega}=\frac{1}{V_{s}}\left|\int_{V_{s}}[b(r)-\bar{b}] e^{i \mathbf{Q} \cdot \mathbf{r}} d \mathbf{r}\right|^{2}
$$

In what follows, since we will be dealing with isotropic objects, we will simplify our treatment by considering both the position vectors and wavevectors as orientationally averaged, scalar quantities.

Let us consider that the material under consideration contains two distinct regions, consisting of a small scale object with an average $b(r)=\left\langle b_{0}>\right.$ which is embedded with homogeneous background characterized by a scattering density $b(r)=<b_{\text {hom }}>$. In this simple case, the sample-averaged scattering density shows a significant spatial dependence, which can be written as

$$
\begin{aligned}
b(r) & =<b_{\text {hom }}>(1-S(r))+<b_{0}>S(r) \\
& =<b_{\text {hom }}>+\left(<b_{0}>-<b_{\text {hom }}>\right) S(r)
\end{aligned}
$$

where $S(r)$ stands for a step function having unit value for nuclei within the scattering body and zero for those forming the homogeneous background. The scattering amplitude for this simple case is written as,

$$
A(Q)=(2 \pi)^{3}<b_{h o m}>\delta^{3}(Q)+\left(<b_{0}>-<b_{h o m}>\right) \int_{V_{s}} S(r) e^{i Q r} d^{3} r
$$

where $(2 \pi)^{3}<b_{\text {hom }}>\delta^{3}(Q)$ stands for a forward scattering quantity which is usually indistinguishable from the transmitted beam. The second term comprises the relevant quantity to characterize our heterogeneous object. Let us now assume that our sample contains some $N_{0}$ objects of this kind with volume $V_{\text {res. }}$. The small angle scattering 
amplitude can then be written, taking into account the parameters stated above as,

$$
\begin{gathered}
A(Q)=\left(<b_{0}>-<b_{\text {hom }}>\right) \sum_{j=1}^{N_{0}} e^{i Q r_{j}} F_{j}(Q) \\
r_{j}=\frac{1}{V_{\text {res }}} \int_{V_{s}} S(r) d^{3} r,
\end{gathered}
$$

where the latter equation stands for the position of the center of mass and $F(Q)$ is a form-factor for the $j^{\text {th }}$ diffracting object. The total differential cross-section can thus be written as,

$$
\frac{\mathrm{d} \sigma}{\mathrm{d} \Omega}=|A(Q)|^{2}=N_{0}\left(<b_{0}>-<b_{h o m}>\right)^{2}\left(\frac{1}{N_{0}} \sum_{j, k=1}^{N_{0}} e^{i Q\left(r_{j}-r_{k}\right)} F_{j}^{*}(Q) F_{k}(Q)\right)
$$

where the asterisk stands for complex conjugate. The equation can be further simplified if we consider an average, normalized form factor $\bar{F}(Q)$ and yields,

$$
\frac{\mathrm{d} \sigma}{\mathrm{d} \Omega}=N_{0} V_{0}^{2}\left(<b_{0}>-<b_{\text {hom }}>\right)^{2} I(Q)|F(Q)|^{2}
$$

where

$$
I(Q)=\frac{1}{N_{0}} \sum_{j, k=1}^{N_{0}} e^{i Q\left(r_{j}-r_{k}\right)}
$$

describes the interference patterns for waves scattered from the $N_{0}$ diffracting centres; each one has a diffraction pattern specified by the normalized form-factor $F(Q)$. In other words, the latter quantity contains information about the shape, size, and structure of the heterogeneities and as such may be evaluated from different geometrical models. Once the $I(\mathbf{q})$ curve has been measured, the task is to fit it with different models in order to find which one best represents the measured data. For instance, in the simple case of an homogeneous spherical isolated aggregate with $a$ radius, the form factor curve will follow

$$
\bar{F}(Q)=\left(3 \frac{\sin (q a)-q a \sin (q a)}{(q a)^{3}}\right)^{2}
$$

In general terms, the description of a given object can be performed in terms of probability density functions, and in such cases $\bar{F}(Q)$ becomes a characteristic function which may be expressed in terms of cumulants $k_{l}$ by means of some cumulant generating function $\kappa(Q)$ so that the form factor simply becomes,

$$
\bar{F}(Q)=\sum_{l=0}^{\infty} k_{l}(i Q)^{l} / l
$$

where the cumulants $k_{0}=0, k_{1}=\left\langle z>, k_{2}=<z^{2}>-<z\right\rangle^{2}, \ldots$ are expressed in terms of the moments $\left\langle z^{n}>\right.$ of the probability density function describing the body under consideration. In fact, since such cumulants are evaluated with respect to the centre of mass, only the even-order terms are relevant since all odd-order terms will cancel out. The most important cumulant is, therefore, $k_{2}=<z^{2}>$ which may be replaced by the 
quantity $R_{g}^{2} / 3$ after further orientational averaging, where $R_{g}$ stands for the radius of gyration, defined as

$$
R_{g}^{2}=\frac{\sum_{i} b_{i} d_{i}^{2}}{\sum_{i} b_{i}}
$$

where $b_{i}$ is the elementary scattering "masses" at a distance $d_{i}$ from the "centre of gravity". This is only valid for scattering at rather small angles, such that $R R_{g} \ll 1$ holds. The associated intensity is then

$$
I(Q)=I_{0} e^{-\frac{q^{2} R_{g}^{2}}{3}}
$$

From plots $\ln (I(Q))$ vs $Q^{2}$, and provided that the Guinier law can be applied, the slope of the linear section gives information on $R_{g}^{2}$. Then, if the shape is known, $R_{g}$ can be

related to specific geometrical parameters, which in the case of spheres yields $a=\sqrt{5 / 3} R_{g}$. This approximation, amounts to neglect the fourth and higher order cumulants, which become relevant for the case on non-spherical objects. Notice, however, that the Guinier approximation does not take into account some subtle features, which can be seen from a plot of the form factor of a sphere as given by Eqn. 4.33. especially at both of the wings of the distribution as well as the subsidiary weak maxima that appear for $Q a \approx 5.5$. It is also worth pointing out that for the case of $Q R_{g} \geq 3$, an expansion of $\bar{F}(Q)$ in powers of $Q$ becomes inadequate and in such as case, as pointed out by Porod (1982), the form factor asymptotically behaves as $Q^{\prime 2}$. In fact, for larger values of $Q R_{g}$, another limit can be taken and the scattering cross-section for particles of surface area $S$ simply becomes

$$
\frac{d \sigma}{d \Omega} \propto\left(<b_{0}>-<b_{h o m}>\right)^{2} S / Q^{4}
$$

so that a plot of $Q^{4}(d \sigma / d \Omega)$ versus $Q^{2}$ will, in the limit $Q \rightarrow 0$, provide information of the specific surface of particles per unit volume (Porod plot).

Whenever heterogeneities are present in concentrations large enough so that the average interparticle separation approaches $\pi / Q$, interference phenomena show up in the scattered intensity, which is now modulated by an interparticle structure factor $S(Q)$. The observed scattering will thus be a product of the particle form-factor and the structure factor, that may show a well-defined peak. In such a case, modeling of the structure factor can be achieved by recourse to statistical mechanics theories of simple hard-sphere liquids, such as the Percus-Yevick equation or the various hypernetted chain approximations.

\subsubsection{Experimental setting}

SANS measurements were done at the ILL D11 instrument. Because of the large cold neutron capture cross-section of ${ }^{10} \mathrm{~B}$ nuclei and the minimum sample thickness available, $1.1 \mathrm{~mm}$, it was decided to use powder samples and fill $0.5 \mathrm{~mm}$ thick cells. The disadvantage of using powder in a SANS experiment is that the recorded signal comes from every grain/air interface, and may hide the information coming from the bulk of the grains themselves. 


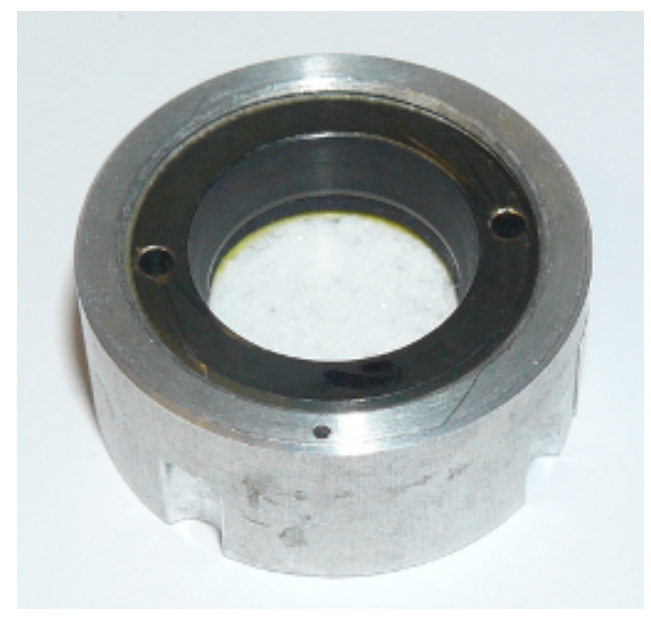

Figure 4.16: D11 cell filled with crushed glass

\begin{tabular}{cc}
\hline Conditions & ambient \\
Sample diameter & $8 \mathrm{~mm}$ \\
Powder thickness & $0.5 \mathrm{~mm}$ \\
Neutron wavelengths & $6 \AA$ and $13 \AA$ \\
Detector distance to sample & $1.2 \mathrm{~m}, 8 \mathrm{~m}$ and $39 \mathrm{~m}$ \\
\hline
\end{tabular}

Table 4.7: Experimental details pertaining to the D11 experiment characteristics.

Pristine and irradiated samples were crushed using a mortar and pestle. The sample cells were made of two quartz windows separated by a $0.5 \mathrm{~mm}$ thick polytetrafluoroethylene (PTFE) spacer. The beam size was adjusted so that no neutrons were interacting with the PTFE. In addition to the measurements on samples, calibration runs were performed for the instrument background, sample container, etc. From Fig. 4.16, one can see an aluminium cell filled with crushed glass. The brown ring is bolted in the aluminium part to press the windows, spacer, and powder together. Measurement characteristics are gathered in Table 4.7. Special care was taken for active specimens to avoid contamination. Glass crushing and crucible filling was done in a glove box by an ILL agent specialized in the handling of contaminating elements.

\subsubsection{Results}

\section{Pristine glasses}

SANS intensities as measured for the four pristine glasses are shown in Fig. 4.17. The first point to be addressed concerns the strong rise at low Q for all the materials. This feature had already been observed in a test experiment on Borofloat and N-ZK7 with $1 \mathrm{~mm}$ thick cells and ascribed to grain scattering resulting from the powder sample. For the measurements shown here, we tried to minimize this feature by choosing a thinner $0.5 \mathrm{~mm}$ thick cell, which obviously was not enough for such a scattering to disappear. To 


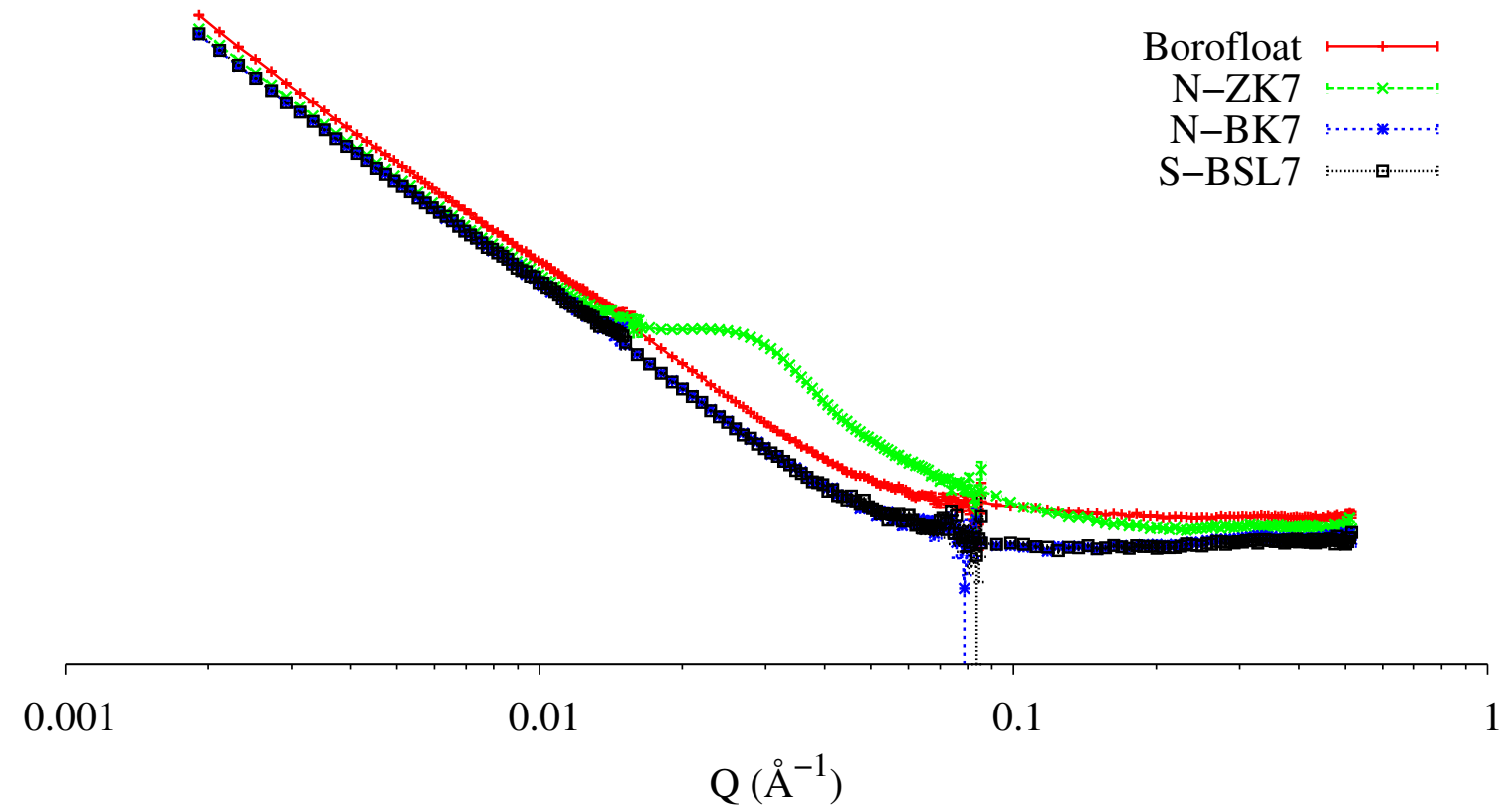

Figure 4.17: SANS intensity of the pristine glasses

\begin{tabular}{cccccc}
\hline & Borofloat & \multicolumn{2}{c}{ N-ZK7 } & N-BK7 & S-BSL7 \\
& pristine & pristine & $3^{\text {rd }}$ dose & pristine & pristine \\
\hline $\mathrm{m}$ & 4.04 & 4.17 & 4.11 & 4.1 & 4.1 \\
$\mathrm{a}$ & $2.80 \cdot 10^{-7}$ & $8.90 \cdot 10^{-8}$ & $1.32 \cdot 10^{-7}$ & $1.14 \cdot 10^{-7}$ & $1.04 \cdot 10^{-7}$ \\
$\mathrm{bkg}$ & 0.033 & 0.024 & 0.047 & 0.017 & 0.016 \\
\hline
\end{tabular}

Table 4.8: Coefficient for the power law fitting of the SANS signals at low Q.

account for such effects we have modelled these ramps using a power law,

$$
I(Q)=a \cdot|Q|^{-m}+b c k g
$$

and those fitted parameter values are gathered in Table 4.8. Such data treatment was made with SASView software [94]. The fact that the slope in this Q-range is around $Q^{-4}$ supports the conclusion that this intensity comes from the interfaces between the air and the glass grains making the powder.

The second and most important point to be mentioned on these measurements is the feature appearing for N-ZK7 around $0.03 \AA^{-1}$, which confirms the existence of aggregates within the material. This should be related to the contrast detected by TEM and STEM. Several fits have been done in order to analyse such a Q-region. First of all, Guinier's law was tried and yielded a value for the radius of gyration, $R_{g}=64 \AA$. However, the very small angle condition $\left(R_{g} \cdot Q_{\max } \ll 1\right)$ was not fulfilled as $R_{g} \times Q_{\max }=2.4$. 


\begin{tabular}{|c|c|c|}
\hline Q range & $0.005 ; 0.013]$ & {$[0.013 ; 0.04]$} \\
\hline Models & Sphere & Sphere \\
\hline Structure factor & Hard sphere & Hard sphere \\
\hline Radius (nm) & 10 & 10 \\
\hline Background & 33.5 & 1.2 \\
\hline Scale factor & 0.4 & 0.6 \\
\hline Volume fraction & 0.39 & 0.24 \\
\hline $\begin{array}{c}\text { Polydispersity } \\
\text { ratio } \frac{\sigma}{\text { Radius }}\end{array}$ & 0.94 & - \\
\hline
\end{tabular}

Table 4.9: Coefficient of the hard sphere fit of the SANS signals of pristine N-ZK7.

The second method used consisted in fitting with a sphere model [51],

$$
I(Q)=\frac{\text { scale }}{V} \cdot\left[\frac{3 V(\Delta \rho)(\sin (q r)-q r \cos (q r))}{(q r)^{3}}\right]^{2}+b c k g
$$

combined with a hard sphere structure factor [80]. To improve the fit process, the contribution from the powder interfaces was subtracted, using the power law and parameters from Table 4.8. The resulting curve is shown in Fig. 4.18a for the Q range of interest. One can see that there are two trends in the black curve, respectively below and above $0.013 \AA^{-1}$. Fitting from $0.005 \AA^{-1}$ to $0.04 \AA^{-1}$ was tried with the hard sphere model combined with a polydispersity (PD) factor. Such a feature accounts for the particles' radius to be distributed around a mean value with a given spread, rather than having a single value. Here, we choose a Gaussian distribution where the width can be adjusted. The PD ratio, which characterizes the Gaussian distribution, corresponds to the ratio of its standard deviation by its central position. One can see on Fig. 4.18b that the modelling curve (in green) does not match the broad peak around $0.025 \AA-1^{-1}$.

In consequence, we have tried to portray the SANS signal as arising from either monoor polydisperse spheres. As a matter of fact, for momentum-transfers from $0.005 \AA^{-1}$ to $0.013 \AA^{-1}$, the curve was fitted with a model containing a polydispersity factor; the results can be seen in Fig. $4.18 \mathrm{c}$. In turn, from $0.013 \AA^{-1}$ to $0.04 \AA^{-1}$, the fitted curve corresponds to a monodisperse sphere population, with results shown in Fig. 4.18d. The relevant model parameters are reported in Table 4.9 .

From the data and arguments given above one can see that the fitted curves reasonably account for the scattering signal. Considering the relative simplicity of the suggested models, in combination with the electronic microscopy observations, we could interpret the SANS signal in pristine N-ZK7 as arising from aggregates of various sizes with a predominance of $10 \mathrm{~nm}$ radius spheres. If the particle size does not seem fully coherent with the TEM image (Fig. 3.3b p.54), this could be validated by the STEM image (Fig. 3.4a p.56), which is done at a higher resolution. 


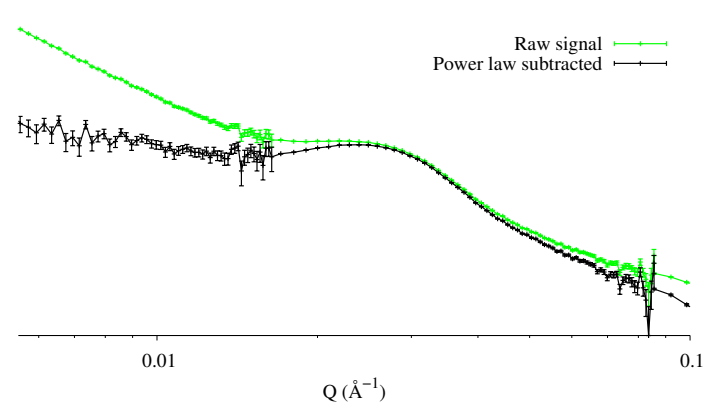

(a) Raw signal or after subtraction of low $Q$ power law.

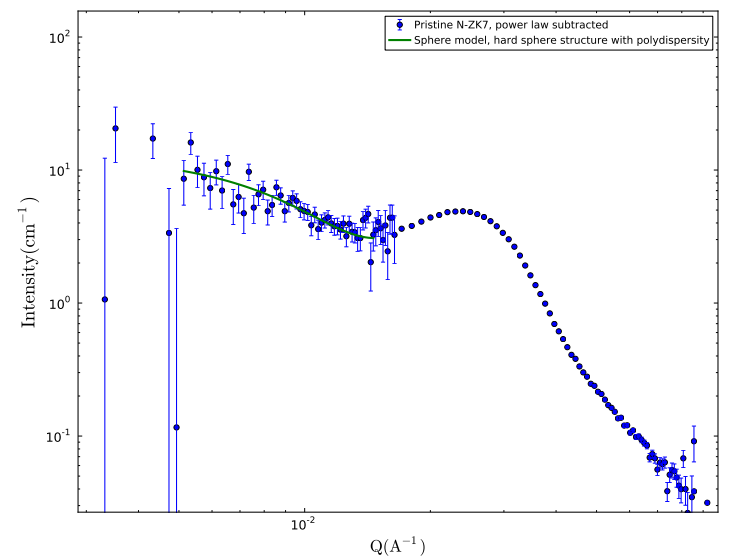

(c) Fitted curve (green) in at small Q, with polydispersivity.

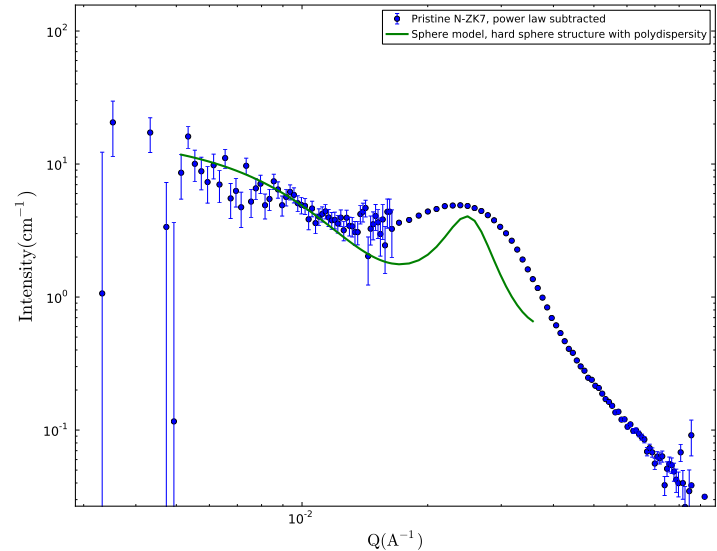

(b) Fitting curve (green) in Q-range of interest, with polydispersivity.

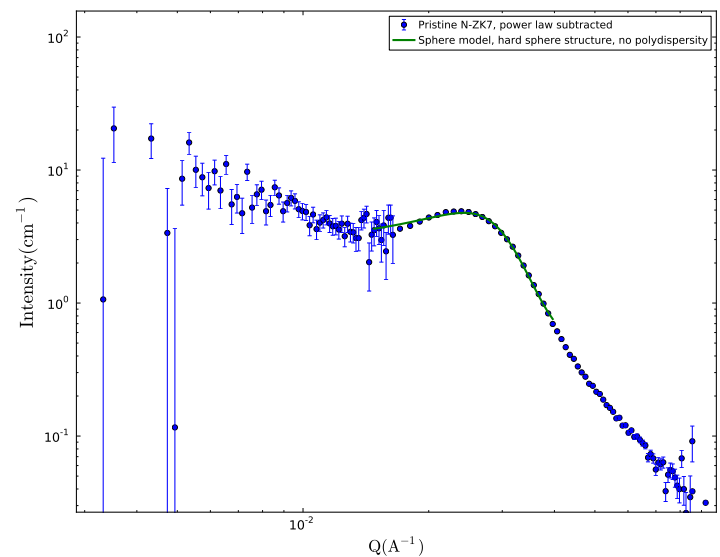

(d) Fitted curve (green) at high Q, without polydispersivity.

Figure 4.18: SANS intensity of pristine N-ZK7 glass. 


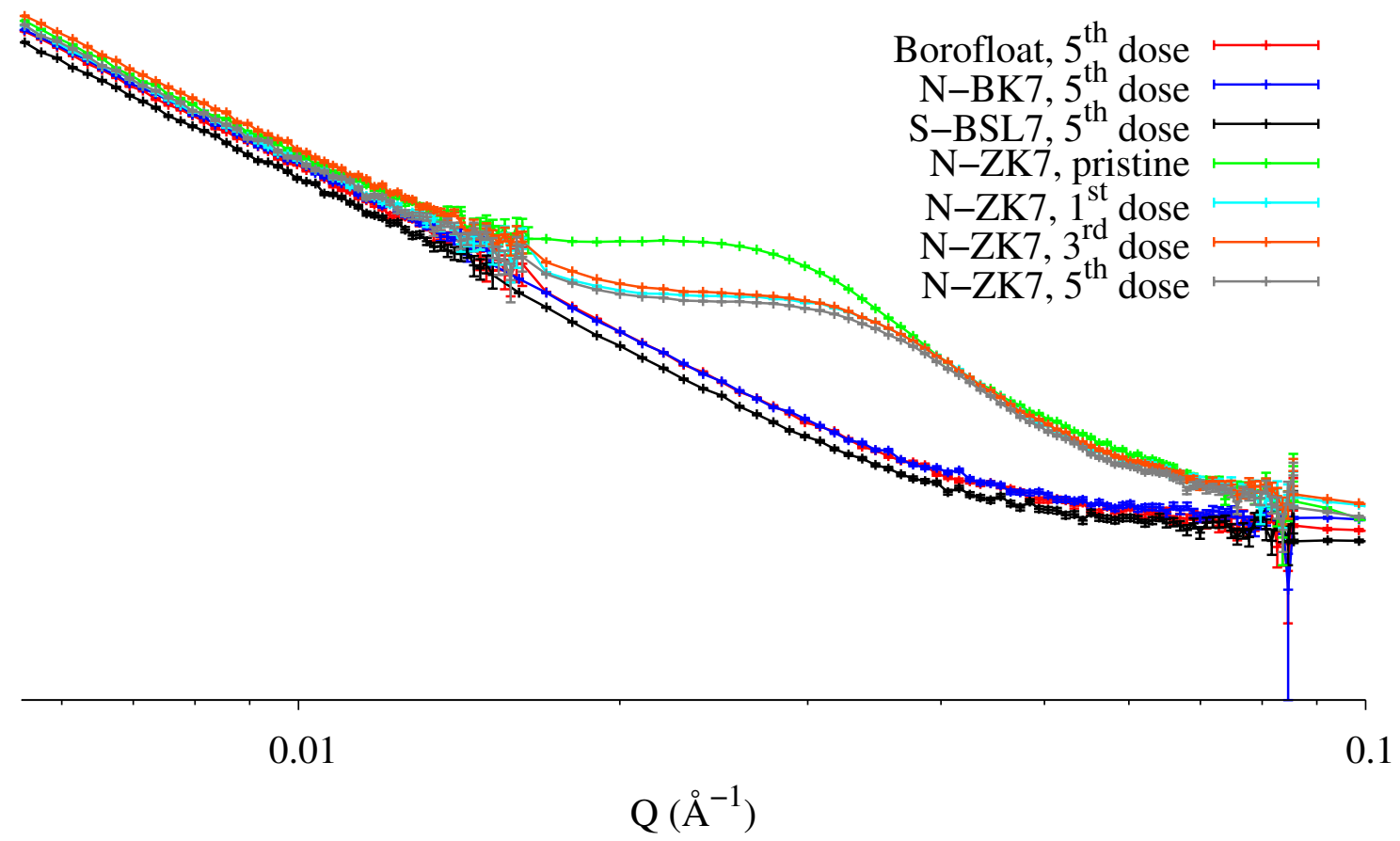

Figure 4.19: SANS signal for irradiated glass powders, D11 instrument (ILL).

\section{Irradiated glasses}

Borofloat, N-BK7, and S-BSL7 curves for the fifth dose are reported in Fig. 4.19, As can be observed, there are no significant differences of the SANS intensity and shape resulting from irradiation. In contrast, the same figure shows that N-ZK7 is noticeably modified due to neutron irradiation. A glance there shows that the shoulder is shifted towards higher Q and seems to decrease in intensity. However, it must be noticed that the signal does not change much going from the first to the fifth dose. This seems to indicate that the new equilibrium state is already reached at the first dose.

Data treatment for N-ZK7 was done following the same protocol as that was employed for the pristine glasses. It yielded similar power law parameters, which are reported in Table 4.8. After subtraction of the power law contribution, two regimes appear below and above $0.018 \AA^{-1}$, respectively, which could be related to hard spheres with or without a distribution of their radius, respectively. Fitting results are gathered in Table 4.10 from which two points merit to be remarked. First, the sphere radius decreases for both $\mathrm{Q}$ ranges. Second, the polydispersity ratio increases due to irradiation. This could be interpreted as a modification of the aggregate population, which transforms into heterogeneities having smaller sizes and wider distributions. In addition, the fact that the curve does not evolve with increasing neutron fluence could mean either that the equilibrium state is reached before completion of the $1^{\text {st }}$ dose, or that the process is not lead by neutron irradiation. The feature does not seem to be correlated to the density evolution of the glass.

In summary, we only found enough scattering contrast for the N-ZK7 sample, which enabled us to derive some values for structural parameters. This comes into line with the 


\begin{tabular}{|c|c|c|}
\hline $\mathrm{Q}$ range & $0.005 ; 0.018]$ & $0.018 ; 0.05$ \\
\hline Models & Sphere & Sphere \\
\hline Structural factor & Hard sphere & Hard sphere \\
\hline Radius (nm) & 7.7 & 8.5 \\
\hline Background & 2.4 & 0.9 \\
\hline Scale factor & 0.4 & 0.2 \\
\hline Volume fraction & 0.43 & 0.22 \\
\hline $\begin{array}{c}\text { Polydispersity } \\
\text { ratio } \frac{\sigma}{\text { Radius }}\end{array}$ & 1.1 & - \\
\hline
\end{tabular}

Table 4.10: Coefficient of the hard sphere fits of the SANS signals for irradiated $\left(3^{\text {rd }}\right.$ dose) N-ZK7

TEM/STEM observations reported before, which also have shown the presence of heterogeneities for such a sample. In other words, the reduction in size of the heterogeneities for this material resulting from radiation effects appears to be clear. This contrasts with results for Borofloat, N-BK7, and S-BSL7, which do not show any remarkable feature either in radiated or native states apart from the broad decaying signals, which are difficult to separate from the inter-grain scattering effects.

\subsection{Neutron diffraction}

\subsubsection{General principles}

Although glasses are devoid of the long range order characteristic of a crystalline lattice, at microscopic scales there may be a continuous range of structures present within the glassy matrix which comprise networks of structural units as well as, in some cases, structures arising from small occluded crystallites resulting from partial crystallization processes. The description of such structural features can only be made in terms of statistical mechanics quantities, such as the radial distribution function $g(r)$. Strictly speaking, such a function has a pole at $r=0$, and then vanishes at small $\mathrm{r}$-values since atoms cannot be located closer than a given distance. Distances about twice the atomic radii $g(r)$ usually show a strong, single peak which results from nearest neighbour contacts such as bond-lengths, which are thus pretty well defined. Further away from the atom considered as located at $r=0$, there is usually a peak or more complex features arising from next-nearest-neighbour contacts and contains information regarding quantities such as bond-angles. For distances still further away, information about further neighbour shells get increasingly washed out until a limit for large distances yielding $g(r)=1$ is smoothly approached at large $r$ values.

The scattering from a glass is often studied using instruments also employed to perform measurements of powder crystalline materials. The quantity of interest here is the single differential scattering cross-section, which is obtained once the contributions from the sample holder and background have been subtracted to the measured intensity and the data is normalized using a Vanadium standard. For a system of N-like atoms located at 
$i$ sites with scattering amplitude $b_{i}$, this quantity can be written as,

$$
\frac{\mathrm{d} \sigma}{\mathrm{d} \Omega}=\left\langle\left|\sum_{i}^{N} b_{i} \cdot e^{i \mathbf{q} \cdot \mathbf{r}_{i}}\right|^{2}\right\rangle=\left\langle\sum_{i, j}^{N} b_{i} b_{j}^{*} \cdot e^{i \mathbf{q} \cdot \mathbf{r}_{i j}}\right\rangle,
$$

where the brackets \langle\rangle represent averaging over the $i$ sites. In the case of a mono-atomic sample, we have to consider contributions to the scattering intensity,

$$
\begin{array}{ll}
i=j & <b_{i} \cdot b_{j}^{*}>=\overline{b^{2}} \\
i \neq j & <b_{i} \cdot b_{j}^{*}>=<b_{i}><b_{j}^{*}>=\bar{b}^{2},
\end{array}
$$

the first of which represents scattering from single nuclei and therefore does not give rise to interference effects; this is usually referred to as the "self" term. In contrast, the second case contains contributions from different atoms and thus provides information concerning the atomic positions. Such a second term is usually referred to as the "interference" or "distinct" contribution. In terms of the cross-section, such terms arrange into

$$
\begin{aligned}
\frac{\mathrm{d} \sigma}{\mathrm{d} \Omega} & =\bar{b}^{2}\left\langle\sum_{i, j \neq j}^{N} \cdot e^{i \mathbf{q} \cdot \mathbf{r}_{i j}}\right\rangle+\sum_{i}^{N} \overline{b^{2}} \\
& =\bar{b}^{2}\left\langle\sum_{i, j}^{N} \cdot e^{i \mathbf{q} \cdot \mathbf{r}_{i j}}\right\rangle+\sum_{i}^{N}\left(\overline{b^{2}}-\bar{b}^{2}\right)
\end{aligned}
$$

Once the cross-section has been measured and converted into absolute units, the structure factor $-S(Q)$ is easily obtained by normalization by the scattering length. Such quantity, which can be written as,

$$
S(\mathbf{Q})=\frac{1}{N}\left\langle\sum_{i, j}^{N} \cdot e^{i \mathbf{Q} \cdot \mathbf{r}_{i j}}\right\rangle
$$

is directly related to the cross-section by,

$$
\begin{aligned}
\frac{1}{N} \frac{\mathrm{d} \sigma}{\mathrm{d} \Omega} & =\bar{b}^{2} S(\mathbf{Q})+\left(\overline{b^{2}}-\bar{b}^{2}\right) \\
& =\frac{1}{N}\left[\frac{\mathrm{d} \sigma}{\mathrm{d} \Omega}\right]^{\text {coherent }}+\frac{1}{N}\left[\frac{\mathrm{d} \sigma}{\mathrm{d} \Omega}\right]^{\text {incoherent }}
\end{aligned}
$$

The coherent term represents the diffraction from spatially correlated points and therefore is wavevector-dependent. In contrast, the incoherent contribution is isotropic and mostly arises from nuclear-spin effects, as previously mentioned. For a multicomponent system, however, the separation into coherent and incoherent contributions becomes less adequate than that given in terms of "self" and "distinct" parts, since the disorder gives rise to a continuum of intensity which adds up to the incoherence contribution arising from spin effects. In fact, in retaining such a separation, one may write the cross-section simply as,

$$
\frac{\mathrm{d} \sigma}{\mathrm{d} \Omega}=\sum_{i} \bar{b}_{i}^{2}+\sum_{i} \sum_{k} \bar{b}_{i} \bar{b}_{k} S_{i k}(Q)
$$

where the bar indicates an average over nuclei in a given site and $S_{i k}(Q)$ are partial 
structure factors which contain information on the microscopic structure for every pair $i-k$ of atom types. All the relevant structural information is thus comprised within an "interference function",

$$
I(Q)=\frac{\mathrm{d} \sigma}{\mathrm{d} \Omega} \sum_{i} \overline{b_{i}^{2}}-I_{0}=\sum_{i} \sum_{k} \bar{b}_{i} \bar{b}_{k} S_{i k}(Q)
$$

obtained from the cross-section after subtraction of the average density of each atom type. Following with such a decomposition, one gets the structure factors defined in terms of a generalized density $\rho_{i k}$ and the average density for each atom type $\rho_{k}^{0}$ as,

$$
S_{i k}=\int_{0}^{\infty} 4 \pi r^{2}\left[\rho_{i k}-\rho_{k}^{0}\right] \frac{\sin (Q r)}{Q r} d r
$$

For a mono-atomic sample, its local structure is thus fully specified in terms of the Pair Distribution Function (PDF) or Radial Distribution Function $g(r)$,

$$
g(\mathbf{r})-1=\frac{1}{(2 \pi)^{3} \rho_{0}} \int(S(\mathbf{Q})-1) e^{-i \mathbf{Q r}} \mathrm{d} \mathbf{Q}
$$

where $\rho_{0}$ is the atomic density, which because of the overall isotropy of the glass sample becomes,

$$
g(r)-1=\frac{1}{2 \pi^{2} r \rho_{0}} \int_{0}^{\infty} Q(S(Q)-1) \sin (Q r) \mathrm{d} Q
$$

The above equation tells us that the experimental determination of $g(r)$ involves a Fourier sine transform. This means that the resolution (or distance between points) in real space has to satisfy the Fourier sampling condition, which in other terms limits the available resolution to $\propto 1 / Q_{\max }$.

From the PDF, one can calculate the Pair Correlation Functions, $G(r)$,

$$
G(r)=4 \pi r \rho_{0}(g(r)-1)
$$

Generalization of the above equation for the case of a multicomponent system is straightforward. The total pair correlation function, say $G^{D}(r)$, will now be a weighted sum of partial pair correlation functions $g_{i k}(r)$,

$$
\begin{aligned}
G^{D}(r) & =\sum_{i} \sum_{k} \bar{b}_{i} \bar{b}_{k} g_{i k}(r) \\
g_{i k}(r) & =4 \pi r\left(\rho_{i k}(r)-\rho_{k}^{0}\right)
\end{aligned}
$$

Some other quantities which are useful to calculate such as coordination numbers around a given atom can also be defined. In particular, the total correlation function is given by

$$
T(r)=G^{D}(r)+4 \pi r \rho_{0}\left(\sum_{j} \bar{b}_{j}\right)^{2}=\sum_{i} \sum_{k} 4 \pi \rho_{i k}(r)
$$

where $\rho_{0}$ stands for the average density of scattering centres. A function that rises quadratically at large $r$, the integral of which is taken as a function of $r$ can give an estimate of the coordination number of a given atom is called the weighed density distribution 


\begin{tabular}{cc}
\hline$\lambda(\AA)$ & 0.5 \\
$\mathrm{Q}_{\max }\left(\AA^{-1}\right)$ & 23 \\
$\mathrm{~T}(\mathrm{~K})$ & ambient \\
$\mathrm{P}(\mathrm{mbar})$ & $\leq 10^{-4}$ \\
\hline Holder diameter $(\mathrm{mm})$ & 6.8 \\
Powder height $(\mathrm{mm})$ & 50 \\
Powder mass $(\mathrm{g})$ & $\simeq 4$ \\
\hline
\end{tabular}

Table 4.11: D4 neutron diffraction experiment characteristics.

which is simply $r T(r)$ or,

$$
\rho(r)=\sum_{i} \sum_{k} \bar{b}_{i} \bar{b}_{k} 4 \pi r^{2} \rho_{i k}(r)
$$

\subsubsection{Experimental settings}

Neutron diffraction measurements were done on Borofloat and N-ZK7. Glasses were crushed into fine powder with a mortar and pestle in order to fill a vanadium cylindrical sample holder (or cell). The irradiated material came from broken samples of the H112/H21 ageing experiments. Pieces peeling from the splintered surface were taken off and crushed. Their thickness was around $0.5 \mathrm{~mm}$. The experiment was carried out on the D4 instrument at ILL. Table 4.11 lists some parameters concerning the experimental setting. In practical terms, measurements for the instrument background, empty container, and a vanadium standard for normalization purposes were also carried out.

Once the measured data were put into an absolute scale by means of normalization with the vanadium standard and the single-differential neutron scattering cross-section was derived, standard corrections and procedures were followed to calculate the static structure factors from such quantities.

\subsubsection{Results}

\section{Pristine state}

The $S(Q)$ static structure factor for the two pristine glasses are shown in Fig. 4.20a. In addition, the first order difference between the two graphs has been computed and is drawn on the same graph. From these curves, it is possible to derive the corresponding pair distribution functions. The full Q-range $(0-23.6 \AA)$ available has been used to calculate the sine-Fourier transformation, using an appropriate window function to deal with series termination effects. The relationship between such a quantity and the distribution of atomic positions is sketched above in Eqns. 4.50, 4.51, and 4.52. Notice, however, that multicomponent systems such as those we are dealing with require a full set of atomic radial pair distributions for their complete specification, as Eqn. 4.54 exemplifies. This can be done in some favourable cases using the technique of isotopic substitution of either all or part of the components, which grounds itself in the differences in scattering lengths of isotopes from a given chemical element [40]. To the best of our knowledge, this can only 


\begin{tabular}{c|cccc}
\hline & $\mathrm{SiO}_{2}$ & $\mathrm{SiNaO}$ & $\mathrm{SiB} 5 \mathrm{NaBaZrO}$ & $\mathrm{B}_{2} \mathrm{O}_{3}$ \\
\hline $\mathrm{Si}-\mathrm{O}$ & 1.615 & 1.62 & 1.60 & - \\
$\mathrm{B}-\mathrm{O}$ & - & - & $1.4 / 1.6$ & 1.36 \\
$\mathrm{Si}-\mathrm{B}$ & - & - & $2.5-3.1$ & - \\
$\mathrm{Si}-\mathrm{Si}$ & 3.1 & 3.05 & 3.0 & - \\
$\mathrm{B}-\mathrm{B}$ & - & - & $2.4-2.6$ & 2.4 \\
$\mathrm{O}-\mathrm{O}$ & 2.63 & 2.61 & $2.3 / 2.6$ & 2.35 \\
\hline
\end{tabular}

Table 4.12: Computed inter-atomic distances by Reverse Monte Carlo (RMC) simulation from neutron diffraction data [43]. "/" means their are two peaks while "-" means the peak is broad.

be reasonably attempted for relatively simple systems having two or three components at most. Unfortunately, our samples not only are far more complicated than that but also, the dominant component, namely $\mathrm{SiO}_{2}$, does not show large contrast variation (i.e. differences in scattering cross-section for its main isotopes). In fact, the listed scatteringlengths [57 for $\mathrm{Si}\left({ }^{\text {nat }}=0.415,{ }^{28} \mathrm{Si}=0.411,{ }^{29} \mathrm{Si}=0.470,{ }^{30} \mathrm{Si}=0.411\right.$ ), all given in units of $10^{-12} \mathrm{~cm}$, make it difficult to perform first difference measurements using such isotopes. The numbers concerning the oxygen isotopes show even smaller differences in scattering lengths. Recent results, however [120], suggest that some test experiment in pure $\mathrm{SiO}_{2}$ may be done, since the high counting rate of present day diffractometers may enable researchers to get some significant information from there.

Although we cannot attempt to identify all the features appearing in the total radial distributions, we could reasonably attribute the most salient features of the PDF to some relatively well-defined inter-atomic distances. This is feasible mostly due to the wealth of detailed data arising from computer molecular dynamics, or Monte-Carlo simulations (see for instance Ref. [49]), for both the pure silica or boron trioxyde glasses as well as from some preliminary work on more complex networks. On such grounds, Table 4.12 reports these distances for four glasses being either vitreous silica, sodium silicate, borate, or alkali-borosilicate [43]. From these values, one can attribute the intense peak around $1.6 \AA$ to a mixed contribution of $\mathrm{Si}-\mathrm{O}$ and $\mathrm{B}-\mathrm{O}$. The second strong peak, around $2.6 \AA$, corresponds to $\mathrm{O}-\mathrm{O}$ distance. In addition, the broad peaks around 4 and $5 \AA$ are generally attributed, respectively, to $\mathrm{Si}-\mathrm{O}$ and $\mathrm{O}-\mathrm{O}$ with second oxygen neighbours.

\section{Irradiated state}

Structure factors and pair distribution functions for the two irradiated glasses are gathered in Fig. 4.21 a-d. The first order difference from the pristine measurement is also plotted in the different graphs. Looking at the variation of the $P D F$ for Borofloat with radiation dose, one can see that the peak around 1.6 $\AA$, although not displaced, has an intensity that decreases and broadens, which indicates that the populations of $\mathrm{Si}-\mathrm{O}$ and possibly some $\mathrm{B}-\mathrm{O}$ distances are more disperse. Also, the peak located around $2.6 \AA$ is shifted towards shorter distances, which seems to indicate a reduction of the $\mathrm{O}-\mathrm{O}$ 


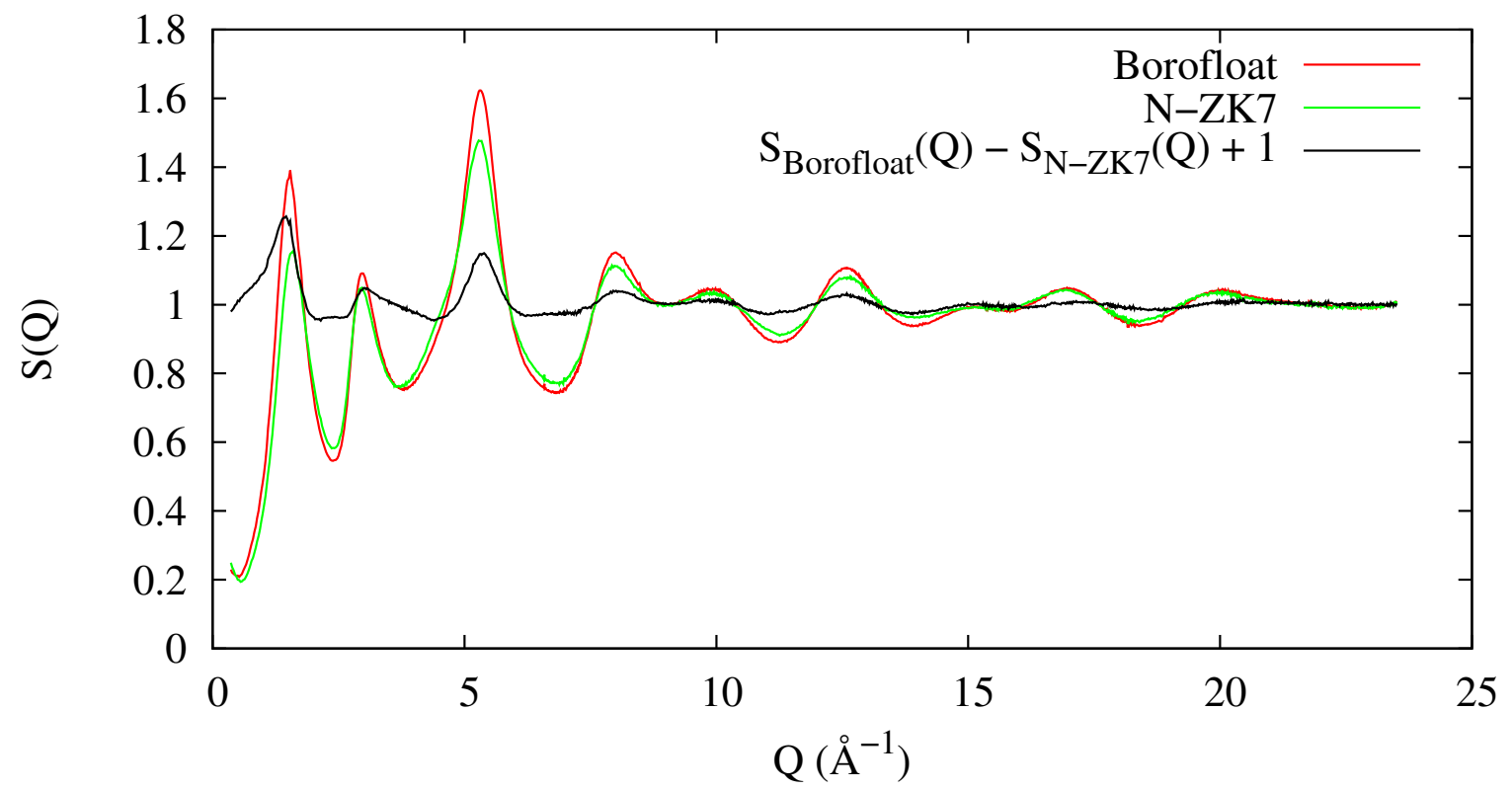

(a) Structure factor $S(Q)$

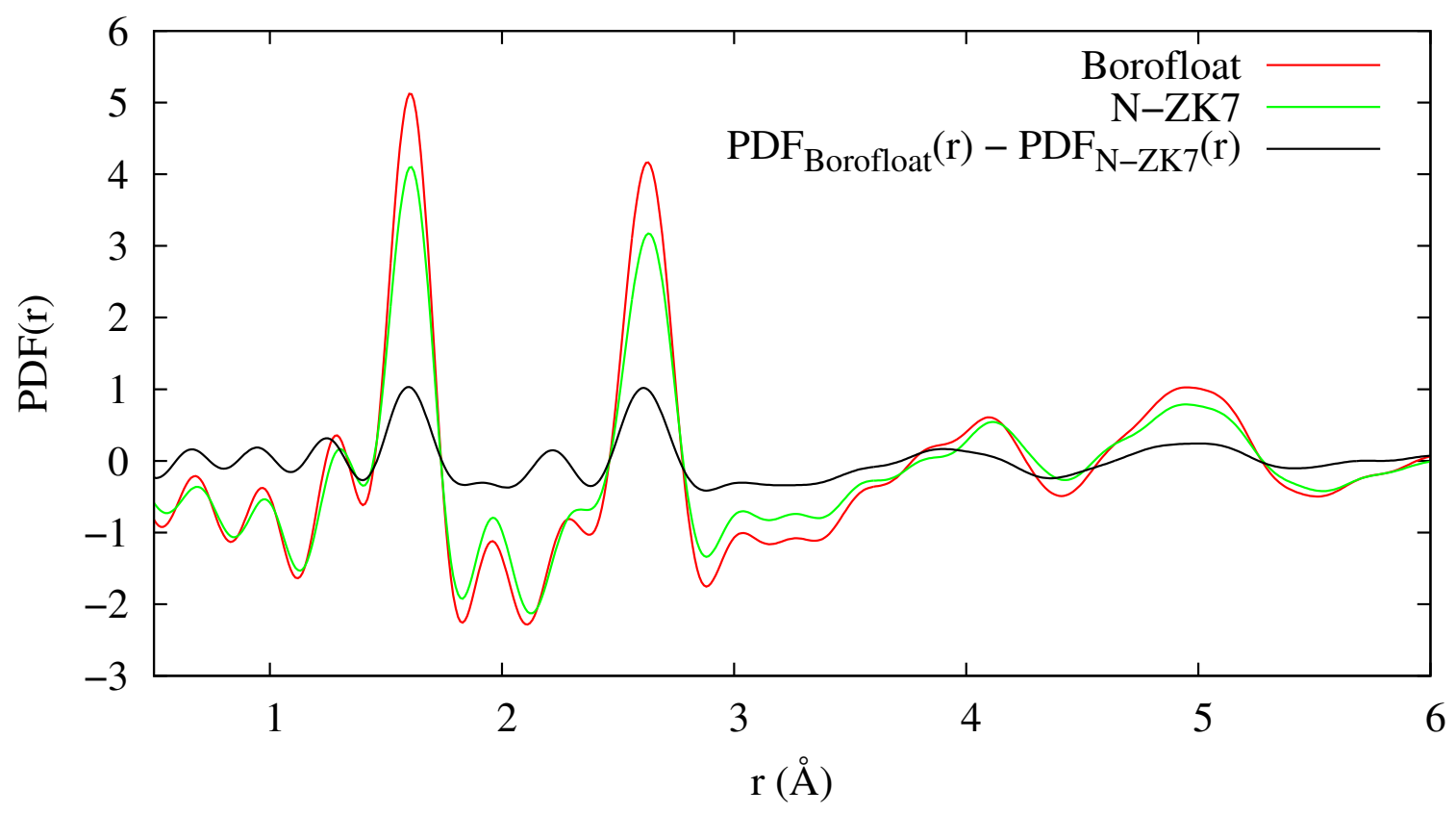

(b) Pair distribution function $P D F(r)$

Figure 4.20: Borofloat and N-ZK7 neutron diffraction signals. 
distance. Perhaps the most interesting modifications pertain to distances around 4 to $6 \AA$, where one can see that the two successive broad peaks are shifted to higher values. Such a range of distances are identified as Range II, comprising distances within 3-5.5 $\AA$, and the beginning of Range III, corresponding to distances beyond some $4 \AA$, in the terminology of A.C. Wright [115]. The former involves the distribution of internal torsion angles, which are those dihedral angles subtended by groups of four atoms connected through chemical bonds. These regions of distances are of particular interest since, as the case of pure $\mathrm{SiO}_{2}$ shows, such angles only show a discrete set of values in the crystalline modifications of the material, whereas a far broader distribution is observed in simulations of the glassy solid. Notice in this respect that access to such regions, together with those distances comprised within Range III, can only be achieved with the help of modelling tools. At any rate, the range of distances where such differences due to radiation become visible mostly correspond to distances between atoms of different tetrahedra.

As regards the changes in the radial distribution concerning N-ZK7, these are less remarkable. In the 4-6 $\AA$ range, for instance, broad peaks appear to be unchanged upon irradiation, which suggests a stability of the atom neighbours at this distance. The peak around $2.6 \AA$ is slightly displaced towards shorter distances, whereas that around $1.6 \AA$ only sees its intensity decrease.

It is possible to make some interpretations from evolutions of Pair Distribution Functions due to irradiation. For instance, the shift of the $2.6 \AA$ peak can be compared to the one occurring for vitreous silica when irradiated with fast neutrons. Such a feature has been attributed to the increase of the three-membered ring concentration associated to a decrease of the average $\mathrm{Si}-\mathrm{O}-\mathrm{Si}$ bond angle [116]. A similar interpretation could be done for the borate network. Bond breaking induced by $(n, \alpha)$ reactions can provoke the re-arrangement of boron rings [38]. It could also affect second and higher neighbour distance and cause a shift the $2.6 \AA$ peak. Specifically for Borofloat, the important shift in the 3.5-5.5 $\AA$ range highlights the important evolution of large elements of the glass network.

\subsection{Interpretation of the results}

Before entering into a discussion on the interpretation of the experimental data presented in this Ph.D. thesis, a disclaimer must be stated. It concerns the nature and characteristics of the materials explored and how much detailed information can be reasonably expected to be derived from analysis of the whole suite of experiments performed here. It is worth pointing out that although borosilicate glasses are extensively employed in a wide variety of technical and industrial applications, which go from nuclear, microelectromechanical systems, to their use as substrates or packaging materials, to their use in basic research for the production of silicon carbide fibre-reinforced inorganic glasses; to our knowledge, no detailed studies on the microscopic structure of these materials have yet been reported. Such a lack of information is not entirely surprising, taking into account the chemical complexity of these materials if compared with pure silica or boron trioxide glasses, the structures of which are only now becoming understood on a fully 


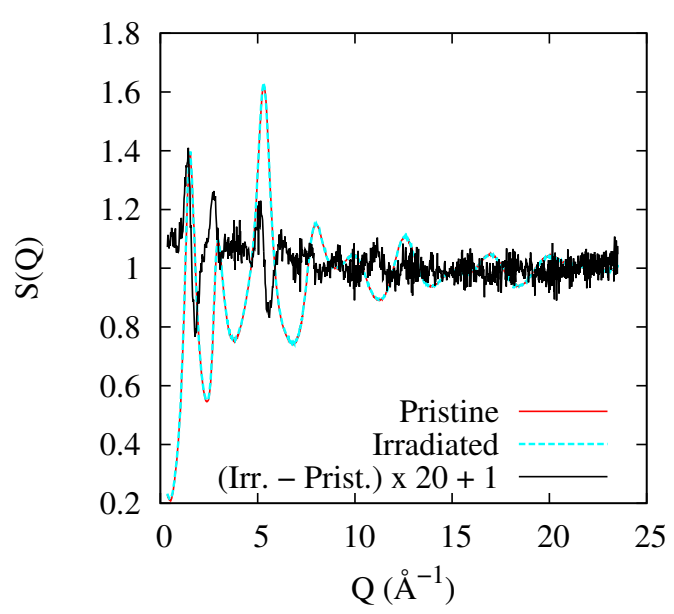

(a) Borofloat, Structure Factor

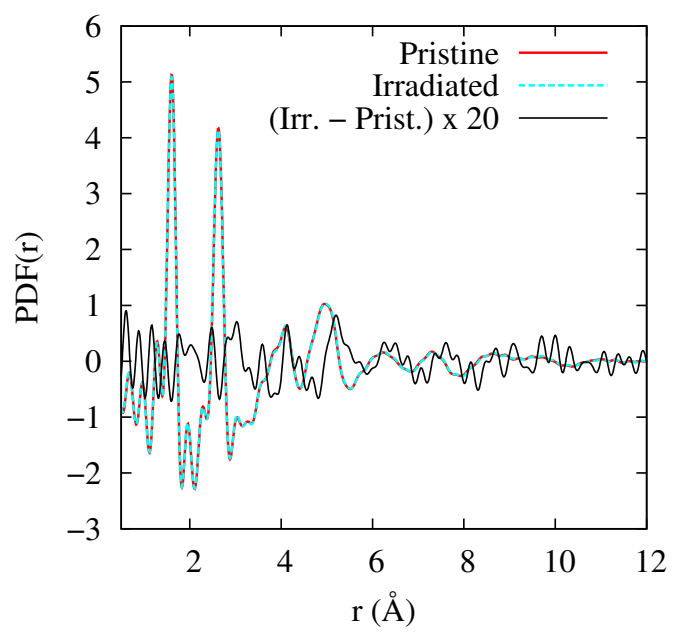

(c) Borofloat, Pair Distribution Function

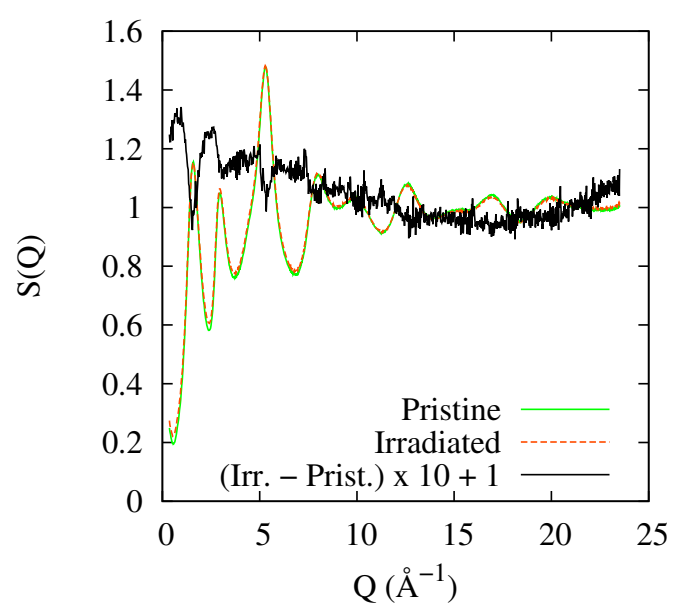

(b) N-ZK7, Structure Factor

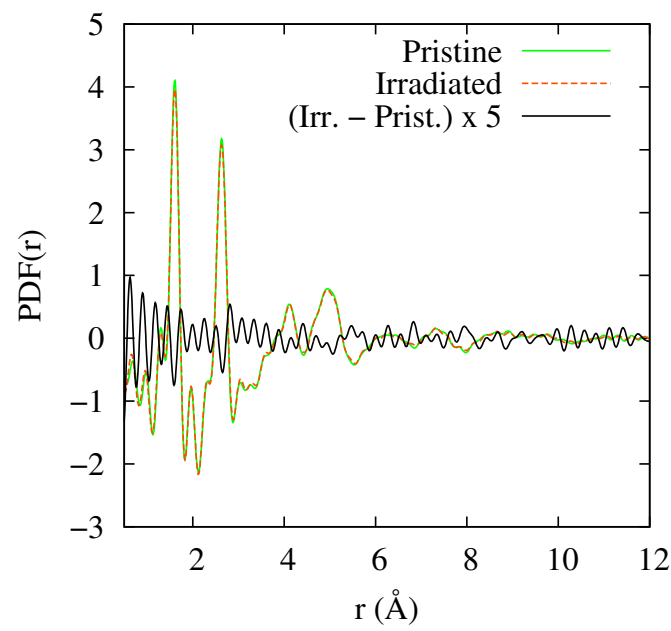

(d) N-ZK7, Pair Distribution Function

Figure 4.21: Comparison of neutron diffraction results on pristine and irradiated Borofloat and N-ZK7, D4 instrument (ILL). 


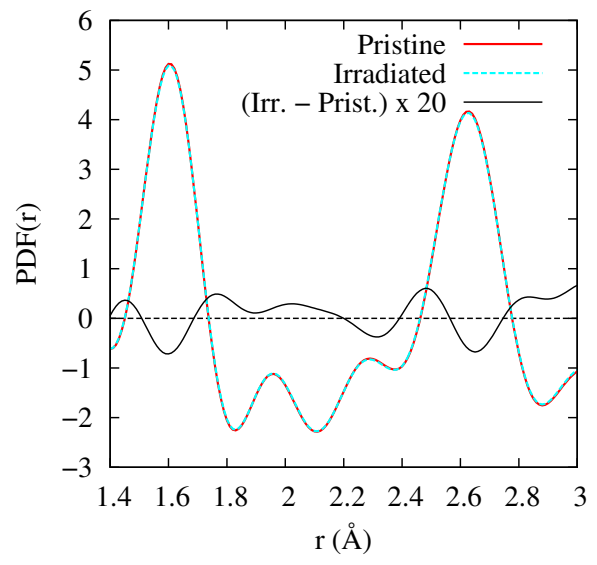

(a) Borofloat

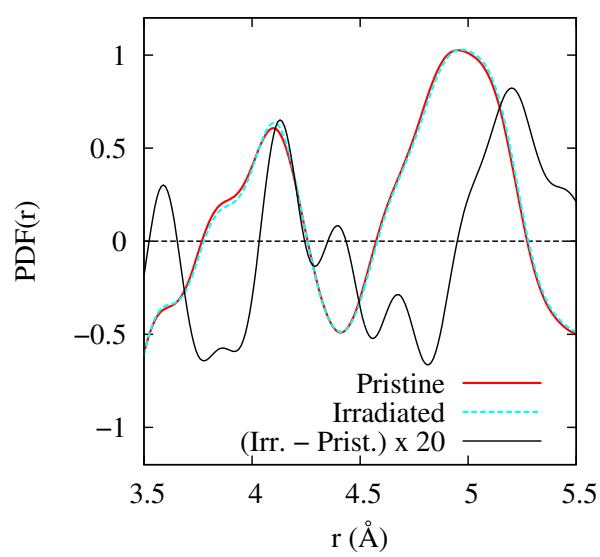

(c) Borofloat

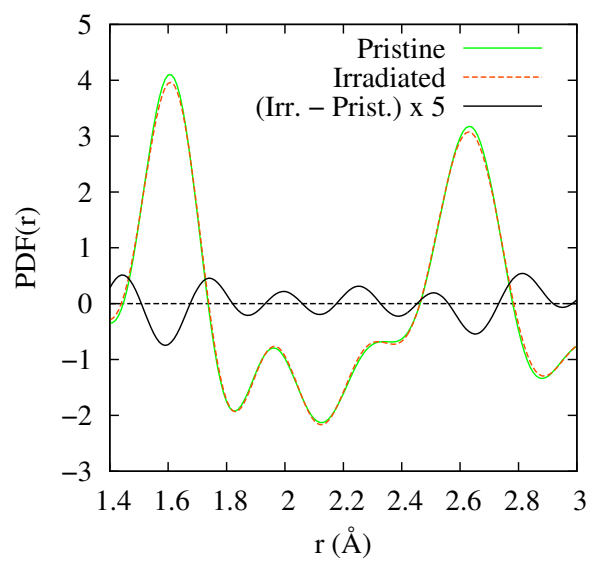

(b) N-ZK7

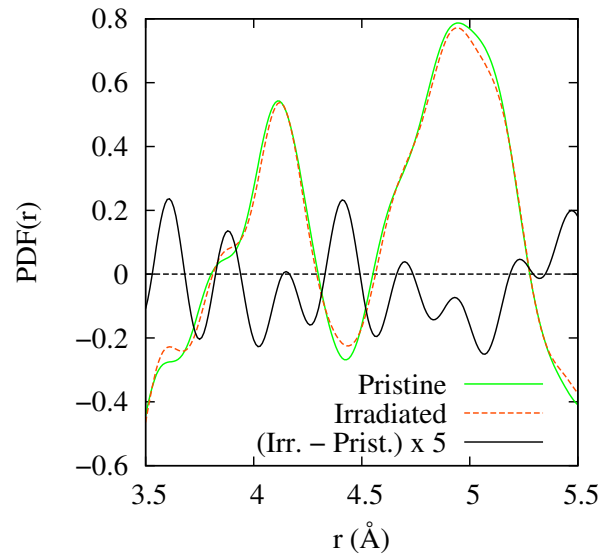

(d) N-ZK7

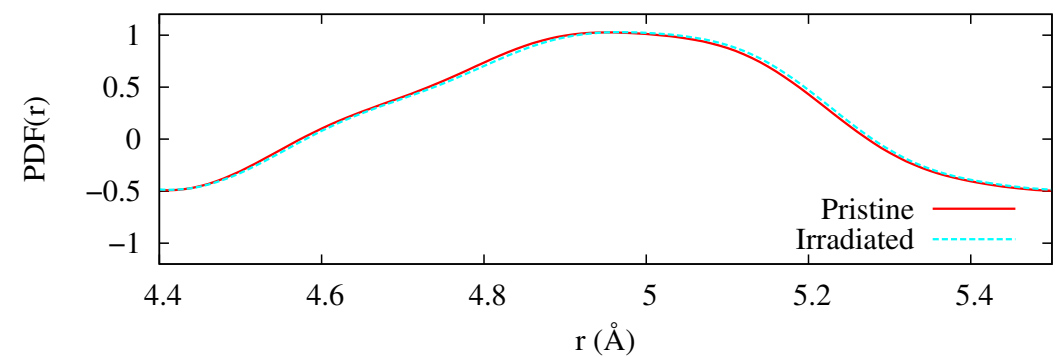

(e) Borofloat

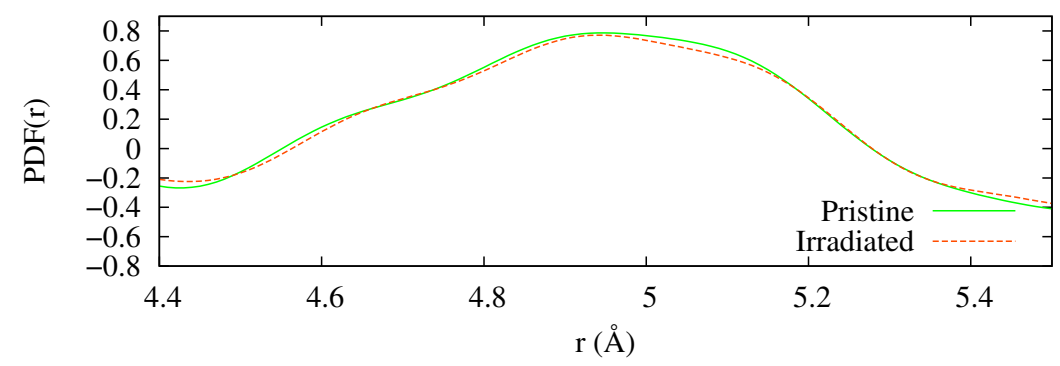

(f) N-ZK7

Figure 4.22: Zoomed views of PDF curves for the two glasses before and after irradiation. 
quantitative basis. Under such circumstances, we are set to explore the most salient features concerning the structure of these materials, whether in pristine or irradiated forms, leaving any attempt to gain finer details on the microscopic structures of these materials for future research.

\subsubsection{Structure of native glass samples}

The first consideration made regarding the set of glass samples studied within this Ph.D script concerns the significant chemical differences between samples. Pertinent details are given in Table 2.1, p 24. Curiously enough, a glance to such tables shows that the boron content for all samples comes to be rather close, and therefore this is something to be discarded in the quest for an explanation for the rather disparate behaviour found for these materials under neutron irradiation. In contrast, the relative amount of the silica network within the various glasses shows larger variations, basically depending upon the content of network modifiers and added ionic species.

In this respect, let us recall that Borofloat shows a remarkably high concentration of network formers, with more than $90 \mathrm{~mol} . \%$ of oxides. In terms of network coordination characteristics, such a large content of network formers yields a picture whether almost all silicon atoms are tetra-coordinated as shown by the $\mathrm{Q}_{4}$ statistic. The low alkali content is effectively reduced due to the fact that it is preferentially bound to aluminium. The resulting structure appears as an entangled network of silica and borate units bridged by $\mathrm{Q}_{4}$ atoms, much like that known to exist in vitreous pure $\mathrm{SiO}_{2}$, intermingled with borate groups mostly in the form of boroxol rings. It is therefore somewhat counter intuitive that no significant phase segregation was detected for this glass using small angle neutron scattering or electron microscopy techniques.

In stark contrast, the N-ZK7 composition shows an alkali concentration almost double of that of Borofloat. From chemical arguments, here we could expect a clear conversion of boron trioxide into tetroxide and an increase in the number of non-bridging oxygens around silicon atoms. Such an expectation seems counterbalanced by the high content of intermediate ions, such $\mathrm{ZnO}$ and $\mathrm{Al}_{2} \mathrm{O}_{3}$, which show concentrations of $10 \mathrm{~mol} \%$ and $4 \mathrm{~mol} \%$, respectively. In addition, previous studies tell us that these elements behave as an alkali sink in order to generate the network. Our results derived from high resolution scanning transmission electronic microscopy have shown that these elements merge together into aggregates of roughly spherical shape and leave the rest of the material with a small content of network modifiers. Such aggregates are further characterized by SANS experiments, which gave an average size for these structures of about $10 \mathrm{~nm}$. Apart from such heterogeneities, the organization of silica and borate networks appears to be remarkably similar to that of Borofloat. In fact, somewhat surprisingly, the distribution of such network-forming units within the material does not seem to be largely affected by the presence of $\mathrm{Zn}-\mathrm{Al}-\mathrm{Na}$ aggregates, as evidenced by the measured Raman patterns, which display characteristics of silicon and boron rich areas. As far as the boron distribution is concerned, the data at hand suggests that in addition to boroxol rings, diborate structures could be present within the material as evidenced by the high $\mathrm{BO}_{4}$ tetrahedra content within the glass. 
The two last glasses, N-BK7 and S-BSL7, should be jointly considered even though their chemical composition is not exactly the same, because they are characterized by similar structural features. Contrary to the glasses previously considered, these last two have both an alkali content sufficiently high to convert the large majority of boron atoms from three- to four-fold coordination. This can be seen from results coming from the measured ${ }^{11} \mathrm{~B}$ MAS-NMR spectra, which show that about $90 \mathrm{~mol} \%$ of the boron units are in tetrahedral environments having four silicon neighbours. Such evidence has been gained through the analysis of peaks appearing in the Raman spectra assigned to the Si-O-Si bond angle bending and stretching band (R-band), which is located around $508 \mathrm{~cm}^{-1}$. To strengthen that point, the presence of an intense peak located around $630 \mathrm{~cm}^{-1}$, which has been attributed to inclusions of danburite/reedmergnerite-like mixed structures, has been spotted. Danburite and reedmergnerite are crystal like-arrangements consisting of two or three silica tetrahedra jointly assembled with two or a single borate tetrahedron respectively. The emergence of such crystal-like arrangements may be rationalized, taking into account the chemical composition of the glass. In fact, the boron/silicon ratio is large enough to create reedmergnerite-like structures and a small amount of danburitelike groups.

Our results show that even though the four glasses belong the alkali-borosilicate family, their structural arrangements split them into two groups. On one side is Borofloat and N-ZK7, which are characterized by silicate and borate rich areas; on the other side is N-BK7 and S-BSL7, within which boron atoms are incorporated into the silica network. Such a difference in structural characteristics gives us a clue to understand the remarkably different behaviour of all these glasses when exposed to thermal neutron irradiation and the resulting ionizing radiation resulting from ${ }^{10} \mathrm{~B}(\mathrm{n}, \alpha)^{7} \mathrm{Li}$ reactions.

\subsubsection{Some microscopic hints to explain the observed macro- scopic behaviour}

As mentioned in the introductory paragraphs of this Ph.D script, the resistance of a neutron guide to radiation-induced mechanical failure is directly correlated with the macroscopic stability of the mirror substrate. Borofloat, which is the glass most prompt to splinter under flux, shows the highest increase in density per received neutron dose, followed by N-ZK7, which also breaks up but requires a higher fluence. The main cause to explain the observed mechanical failure is ascribed to the compaction of the glass. In contrast, the fluence limit for N-BK7 has not yet been reached in the present set of measurements. Its density variation versus received dose is somewhat similar to that exhibited by S-BSL7, which makes these two glasses macroscopically more stable.

From the characterization experiments gathered here, some plausible explanation of the behaviour just described may be put in place. In fact, our own spectroscopic observations tell us that, during irradiation-induced ageing processes, atoms are re-arranged leading to glass structures into somewhat different configurations. Borofloat and N-ZK7 are examples of this process which develops in two well defined steps, namely, the glass 
first quickly shrinks up to a saturation state and then swells, or relaxes, very slowly. The compaction phenomenon can be related to structural characteristics of the vitreous network. In fact, both pristine glasses are characterized by well separated silica and borate networks, a feature that is promptly affected by radiation. In particular, results from Raman spectroscopy show that the silica network compacts, favouring the existence of three-membered silicon rings correlated with a reduction of the Si-O-Si angle. In addition, some boron atoms get incorporated into the silica network. Such rearrangements do not lead to large scale segregation, as proven by SANS measurements. Getting into finer details, we have also found that, in Borofloat, a mixing process involving the two alluded networks takes place. We have followed how the boron environment evolves, modifying the population of its different species. $\mathrm{BO}_{4}$ units, at the origin within the (3Si, 1B) neighbours configuration, are converted into $(4 \mathrm{Si}, 0 \mathrm{~B})$, which leads to the incorporation of boron into the silica network as a consequence of irradiation. We have found that such a feature for Borofloat and N-ZK7 may explain their large shifts of the Raman R-band, which cannot be explained solely as the result of compaction of the silica network. The shrinking of such glasses somehow reach a saturation state beyond which they swell at a very slow rate where the vitreous structure does not appear to undergo further changes. In terms of basic physical causes behind such a behaviour, we attribute the observed trends to the accumulation of point defects with received dose.

The macroscopic stability of N-BK7 and S-BSL7 arises from the sturdiness of their vitreous networks. Contrary to the two other glasses, irradiation does not seem to affect the main structural features. In fact, taking into account the rather noisy Raman spectra for S-BSL7, one could observe that the signatures of the silica and borosilicate networks were stable under irradiation. As a matter of fact, the only relevant change induced by radiation appears to be a modification of the alkali/alkaline-earth distribution leading to the generation of boron trioxide. This leads to a conversion of such ions into network modifiers through the generation of NBOs surrounding silicon atoms. The mechanism is of interest since, in addition to the point defect accumulation, it might explain the swelling of the glass. The rationale for this is grounded on the fact that a $10 \%$ increase of the sodium content of binary sodium-silicate or sodium-borate leads to a density increase two times larger for borate than for silicate. Therefore, relocating alkalis from boron to a silicon environment will generate a net decrease of the overall density.

Summing up these ideas, we have found that the glasses most reactive to thermal neutron irradiation were those showing well defined silica and borate rich areas. The macroscopic behaviour of the glass is driven by the shrinking of the silica domain and the incorporation of boron atoms inside it. Meanwhile, the macroscopically stable glasses have a far more homogeneous vitreous network, thanks to the assembly of boron and silicon atoms into reedmergnerite-like units, a feature that appears to be resistant to irradiation. 


\section{Conclusion}

The mechanical failure of neutron guides due to irradiation has been a known issue by neutron research centres for several decades. A considerable amount of work allowed us to give fluence limits to the different glasses used to manufacture beam lines. Starting from that point, the aim of the work presented here was to explain how this phenomenon manifests itself and why it happens. Here, we focus onto the three glasses most used around the world to produce neutron guides, namely Borofloat, N-ZK7, and N-BK7. To this selection, S-BSL7 was added, as it was foreseen as a possible candidate to future guide renewal programmes.

Thanks to neutron guide simulation software, McStas and MCNP, to help propagate the neutrons in the mirror substrate, it was possible to know at what depth the neutron captures were taking place, which is tantamount to determine the position where the ${ }^{10} \mathrm{~B}(\mathrm{n}, \alpha)$ reaction deposits its energy. This work allowed us to ascertain that mirror cracking was provoked by an heterogeneous ionizing energy deposition on the glass substrate. In more detail, guide walls have a regular thickness around $10 \mathrm{~mm}$ or more, while the neutron captures take place within the first hundred micrometres. Let us mention that the average range of an $\alpha$ particle and a lithium ion in these borosilicates is around $4.7 \mu \mathrm{m}$ and $2.5 \mu \mathrm{m}$, respectively.

Such figures highlighted the need to produce homogeneously irradiated materials in order to characterize how the glass structure evolves, and also why mechanical stress is generated in the case of heterogeneous irradiation. For that purpose, it was necessary to design an new irradiation shuttle optimized for boron containing materials. Such a requirement was dictated by the need to keep the samples below their glass relaxation temperature and also to avoid the melting of the shuttle itself, taking into account the significant amount of heat produced by the above referred neutron capture reactions. The equipment was designed to irradiate $10 \times 10 \times 1.1 \mathrm{~mm}^{3}$ specimens.

By combining the apparatus and an hydrostatic weighing scale, it was found that the glasses most prompt to brittle splintering, Borofloat and, at a lower extent, N-ZK7, were those most sensitive to irradiation. In the fluence range where guide breaking happened, their density increased quickly, while the two other glasses, N-BK7 and S-BSL7, were found to be stable. To further confirm that this phenomenon was generating stress in glass plates, grazing incidence irradiation was done on $0.7 \mathrm{~mm}$ thick Borofloat strips. The $2^{\circ}$ incidence angle, combined with the high macroscopic absorption cross-section, induced a gradient of irradiation and hence shrinking within the plate. Visual inspection showed that stress was generated in the sample, since this lead the samples to develop a marked curvature showing a linear correlation with the received dose to the inverse of its radius. 
The work then focused on the understanding of the mechanisms behind the rather different behaviour exhibited by our glass samples under irradiation. The set of microscopic characterization tools contained Raman and NMR spectroscopy, neutron diffraction, and small angle scattering. These techniques split the glasses into two groups. On one side were Borofloat and N-ZK7, which both feature a rather low content of $\mathrm{BO}_{4}$ units together with silica- and borate-rich areas. Their behaviour under neutron flux is dominated by their silica domains that shrink and incorporate boron atoms inside. On the other side were N-BK7 and S-BSL7, which are characterized by an homogeneous borosilicate network made of mostly $\mathrm{BO}_{4}$ units bonded with four silicon atoms. This feature appears to be quite stable under irradiation and reflects on the macroscopic density of the material. In any case, no phase segregation was detected under flux; the deposited energy and the ballistic mixing tend to create a more homogeneous glass.

This entire study allowed us to understand, in a qualitative way, why neutron guide breakup may occur under irradiation. In a more general way, the irradiation apparatuses developed are now available to further characterize the substrate in the future. On such grounds, one can hope that the methods reported here will be applied later to avoid guide incidents like those that occurred in the past. 


\section{List of Publications}

R. Boffy, M. Kreuz, J. Beaucour, U. Köster, and F.J. Bermejo. Why neutron guides may end up breaking down? Some results on the macroscopic behaviour of alkali-borosilicate glass support plates under neutron irradiation. Nuclear Instruments and Methods in Physics Research B, 358:179 - 187, 2015.

R. Boffy, S. Peuget, R. Schweins, J. Beaucour, and F.J. Bermejo. High thermal neutron flux effects on structural and macroscopic properties of alkali-borosilicate glasses used as neutron guide substrate. EMRS spring meeting 2015 Proceedings, 2016.

J. Beaucour, M. Kreuz, M. Boehm, R. Boffy, V. Cristiglio, B. Demé, L. Didier, P. Falus, P. Fouquet, R. Gandelli, Y. Gibert, B. Giroud, B. Jarry, P. Lachaume, S. Roux, F. Thomas, and A. Vorobiev. The H5 guide system - the latest innovative guide system at the ILL. Neutron News, 26(3), 2015. 


\section{Appendix A}

\section{Neutron guide design}

The Endurance program was launched to pursue the renovation of some of the ILL facilities. This project comes after the Millennium Program that consisted, among other things, in the refurbishment of the H5 guide system.

The beam line renovation program will affect the cold and thermal neutron guides, H15H16 and H23-H24, respectively. On Fig. A.1, one can see the location of those beam lines with respect to the whole ILL guide infrastructure.

The main aim, apart of taking care of some ageing equipment, was to find significant improvements in guide performance resulting from recent developments in neutron guide technology. Getting into details, the purpose was to take advantage of the large divergence in the in-pile area thanks to modern high-m super-mirrors. The beam cross section can thus be expanded and divided to accommodate new guides, which could not be installed in-pile in previous refurbishment works due to a lack of space. This has, as a consequence, the reduction of the beam divergence, an issue we can bear with since most of the instruments cannot use the full angular distribution of an $\mathrm{m}=2$ guide. In the specific case of an inelastic scattering experiment, a focusing nose is usually installed.

\section{A.1 Analytical estimations}

Optimization of the neutron guide performance is usually based on a comparison of the neutron flux between the installed and the proposed new geometry at their exit points. For such an endeavour, we will rely on computer simulations since they will allow us to finely tune the beam line parameters to have the most adapted geometry for the end instruments. However, as a sanity check, it is always advisable to run some analytical estimations of the guide performance in parallel in order to cross-check the results of the simulation and check for possible inconsistencies.

As explained in Chapt 1 on $\mathrm{p} 9$, the beam intensity within a guide is dependent upon its cross section dimensions, radius of curvature, and the m-value of the mirror. Such parameters will set the accepted divergence, filling, and losses by reflections. These three quantities can, in fact, be estimated analytically .

The accepted divergence is directly proportional to the m-value of the coating. Hence, from one design $(i)$ to another $(j)$, the gain coefficient is given by the ratio of the m- 


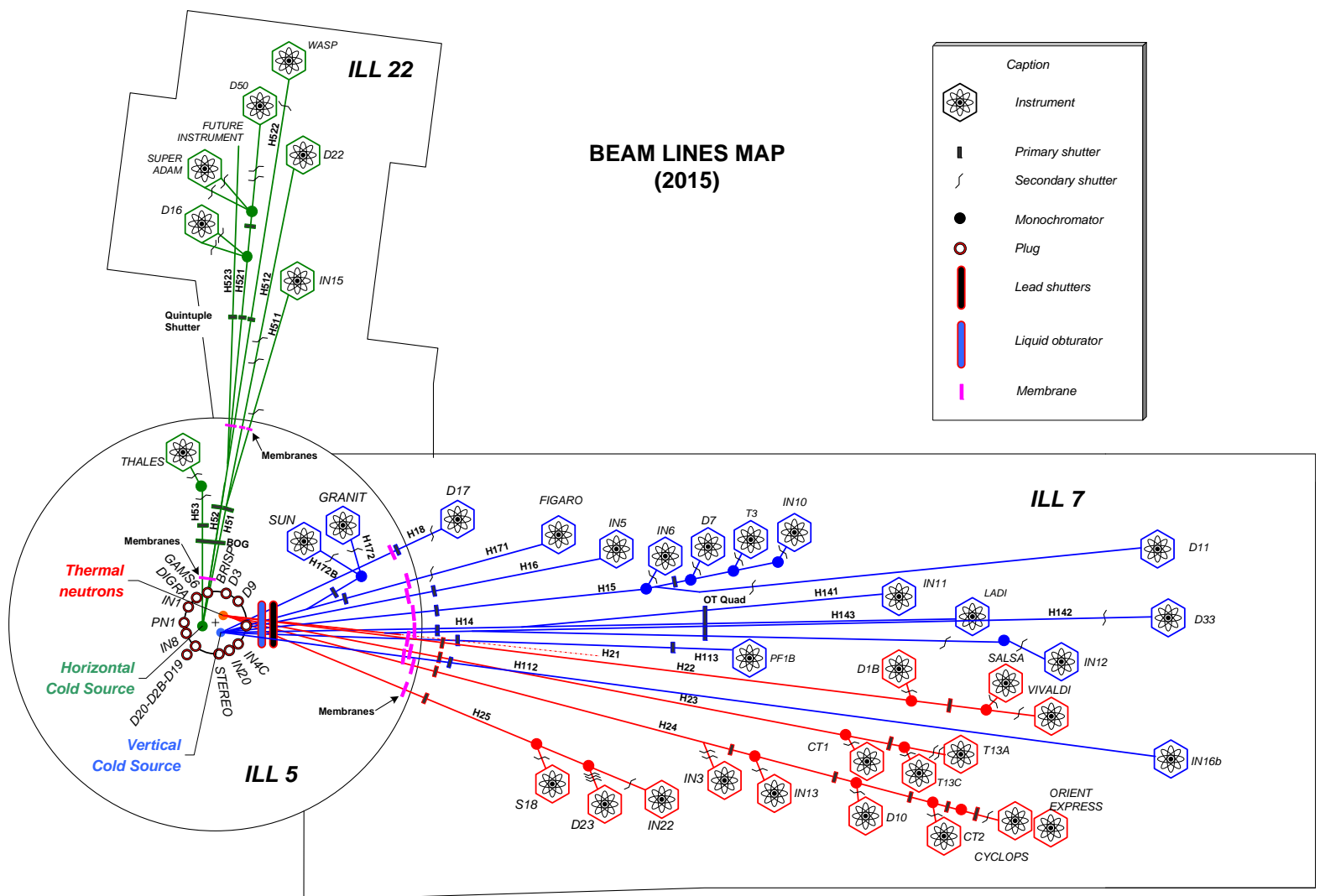

Figure A.1: ILL guide infrastructure. 
values of the vertical and horizontal mirrors, $\mathrm{k}_{\mathrm{m}}=\frac{\left(m_{\text {vert. }} \times m_{\text {horiz. }}\right)_{j}}{\left(m_{\text {vert. }} \times m_{\text {horiz. }}\right)_{i}}$. For a given guide, the filling formulas are given in the same section mentioned previously. Finally, one can estimate the losses due to the bounces by considering the average incidence angle and wavelength of the neutrons to calculate an average number of bounces and compute the loss in intensity thanks to the corresponding reflectivity coefficient of the mirrors. Within the next pages, the flux multiplication factors $\mathrm{k}_{\mathrm{f}}$ and $\mathrm{k}_{\mathrm{r}}$ correspond to the evolution of guide filling and losses by reflections, respectively, from one geometry to the other.

\section{A.2 H15-H16}

\section{A.2.1 Modification of the in-pile section}

When designing neutron transport systems based upon guides, one gets confronted with rather strict constraints imposed by the beam line trajectory. Such restrictions stem from the relatively small critical angle of reflection of thermal or cold neutron on nickel mirrors which for, say, $1.8 \AA$ particles with a $\mathrm{m}=3$ super-mirror, already a high coating for a whole beam line, comes to be about $0.54^{\circ}$. Such a fact sets the average radius of curvature for modern thermal or cold guides at about $20000 \mathrm{~m}$ and $2000 \mathrm{~m}$, respectively [18]. It goes without saying that such a limitation directly impacts the ability to find available space to install newer or refurbished instruments within the modernization programs of a given installation. The super-mirror technology was of great help for such a purpose, since it allows for the increase of the radius of curvature of the neutron guides. In the specific case of the H15-H16 renewal program, we had an idea to slightly change the orientation of the guide entrance in order to gain some extra space between beam lines. In what follows, we will describe our contribution to such a design effort.

In order to simulate the effect of the proposed changes in geometry on the neutron flux, a MCNP neutron source was used instead of the regular McStas counterpart. This new source was created upon the grounds set by the ILL reactor MCNP model that fed the current and the refurbished H15-H16 geometries. It consists on a rectangular source, dimensions $17 \mathrm{~cm}$ by $25 \mathrm{~cm}$ (width by height), located $180 \mathrm{~cm}$ from the guide entrance. It is worth remarking that such a flexible positioning enables us to study matters of current interest within existing or projected sources, such as the optimal location of the guide entrance with respect to the neutron source in order to collect more divergence and thus increase the transported flux. To avoid contamination from unwanted neutrons (i.e. those with far too high energies), the acceptance criterium was made to depend upon the incoming neutron wavelength so that the slower the particle, the higher its divergence could be. Such a rule is driven by the fact that the critical reflection angle increases with wavelength. A McStas simulated view of the in-pile elements is depicted in Fig. A.2, from where one can gauge the cold source, the MCNP generated source, and the first elements of H15 and H16.

The source was used to simulate the rotation of the guide entrance up to $0.4^{\circ}$ along its central vertical axis. The simulation did not show any drastic effects on neutron flux within the guides, see Fig. A.3, that shows the flux wavelength spectrum at $30 \mathrm{~m}$ from the guide start in H15 and H16. The flux for H16 seems to slightly decrease with increasing 


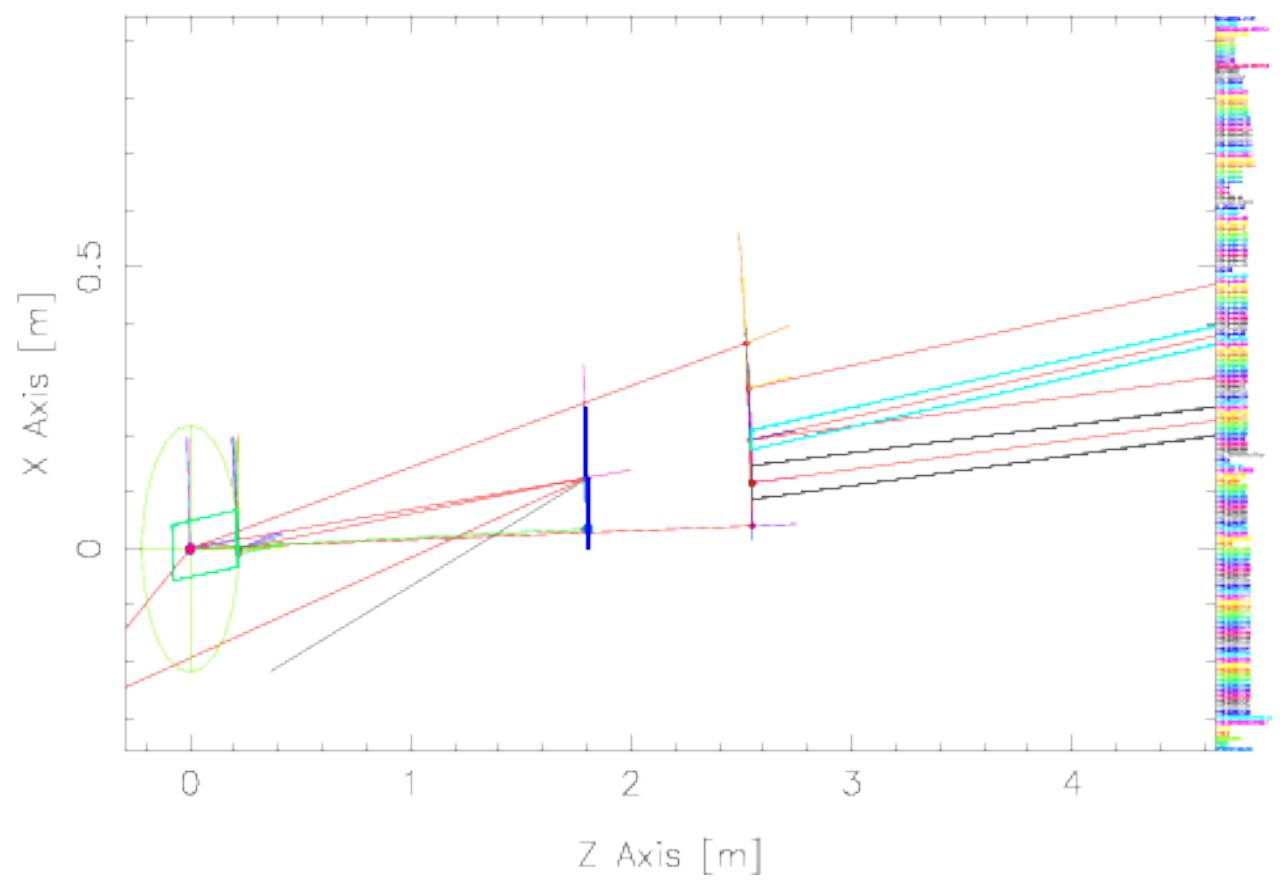

Figure A.2: View from above with McStas of VCS and some guides. Black and light blue polygons are H15 and H16 first guide elements, thick blue line is the implemented MCNP source geometry, and green circle with rectangle inside is the VCS.

the rotation angle, although the variation is negligibly small. This source was also used for all the simulations shown in the following pages of the document.

\section{A.2.2 Performance of instruments}

The final effects of the guide renewal program in terms of neutron flux are now studied for three instruments hosted by H15 and H16, namely D7, D11, and IN5. Their wavelength and angular acceptance is given in Table A.2. As will be discussed below, the guide renewal will probably lead to a relocation of some of the instruments to a different guide in order to free some space for installation of new experiments. The geometries of H15 and H16, installed in 2015, are schematically described in Table A.1. H15 feeds D7 and then splits into two sub-lines, with one $50 \times 30 \mathrm{~mm}^{2}$ line that travels in a rectilinear trajectory down to D11. One must mention that IN6, T3 and IN10 are also fed by such a guide, but they have not been considered for the preliminary study presented here. H16 is currently dedicated to IN5; the last $15 \mathrm{~m}$ are vertically and horizontally focusing the beam down to an exit measuring around $53 \times 14 \mathrm{~mm}^{2}$. Because the design of the focusing guide itself amounts to a considerable workload, we have taken the guide specifications as already set. The flux in current and future geometries has been simulated by assembling the last guide elements with a constant cross-section instead of focusing. In this way, only the efficiency of the upstream guide was tested. The current H15 and H16 guide geometries are shown in Fig. A.4 a-b.

In the configuration foreseen for the near future, H16 will feed D7 and will have an 


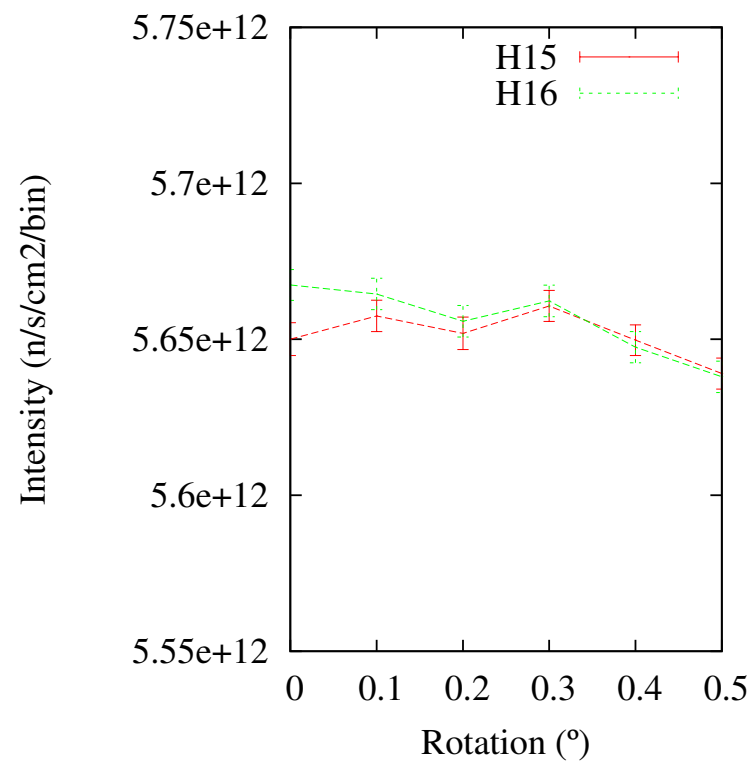

Figure A.3: Effect of guide entrance rotation on flux intensity at $30 \mathrm{~m}$ from the source, wavelength acceptance from 1 to $15 \AA$.

\begin{tabular}{ccccc}
\hline & $\begin{array}{c}\text { Cross-section } \\
\left(\mathrm{mm}^{2}\right)\end{array}$ & $\begin{array}{c}\text { Length } \\
(\mathrm{m})\end{array}$ & $\begin{array}{c}\text { Curvature radius } \\
(\mathrm{m})\end{array}$ & $\begin{array}{c}\text { Mirror coating } \\
(\mathrm{v} \times \mathrm{h})\end{array}$ \\
\hline H15 2015 & $200 \times 50 \rightarrow 45$ & 2.5 & $\infty$ & $\mathrm{m}=2$ \\
& $200 \times 45$ & 5.5 & 2700 & $\mathrm{~m}=2$ \\
& $200 \times 30$ & 49.5 & 2700 & $\mathrm{~m}=1$ \\
& & D7 & $\mathrm{m}=0.65$ \\
& & & $\infty$ & \\
\hline H16 2015 & $200 \times 30$ & 1.25 & $\mathrm{D} 11$ velocity selector & $\mathrm{m}=2$ \\
& $200 \times 30$ & 6 & 2700 & $\mathrm{~m}=2$ \\
& $200 \times 30$ & 21.25 & 2700 & $\mathrm{~m}=1$ \\
$200 \times 30$ & 20.6 & $\infty$ & $\mathrm{m}=1$ \\
$\rightarrow 53 \times 14$ & & $\infty$ & $\mathrm{m}=2 \times 3$ \\
& & IN5 &
\end{tabular}

Table A.1: Schematic description of H15 and H16, installed in 2015.

\begin{tabular}{cccc}
\hline & IN5 & D7 & D11 \\
\hline Wavelength $(\AA)$ & {$[1.5 ; 15]$} & {$[2 ; 6]$} & {$[4.5 ; \infty]$} \\
m-equivalence & $\infty$ & 2.8 & 0.65
\end{tabular}

Table A.2: Neutron beam characteristics for future H15 and H16, intruments. 


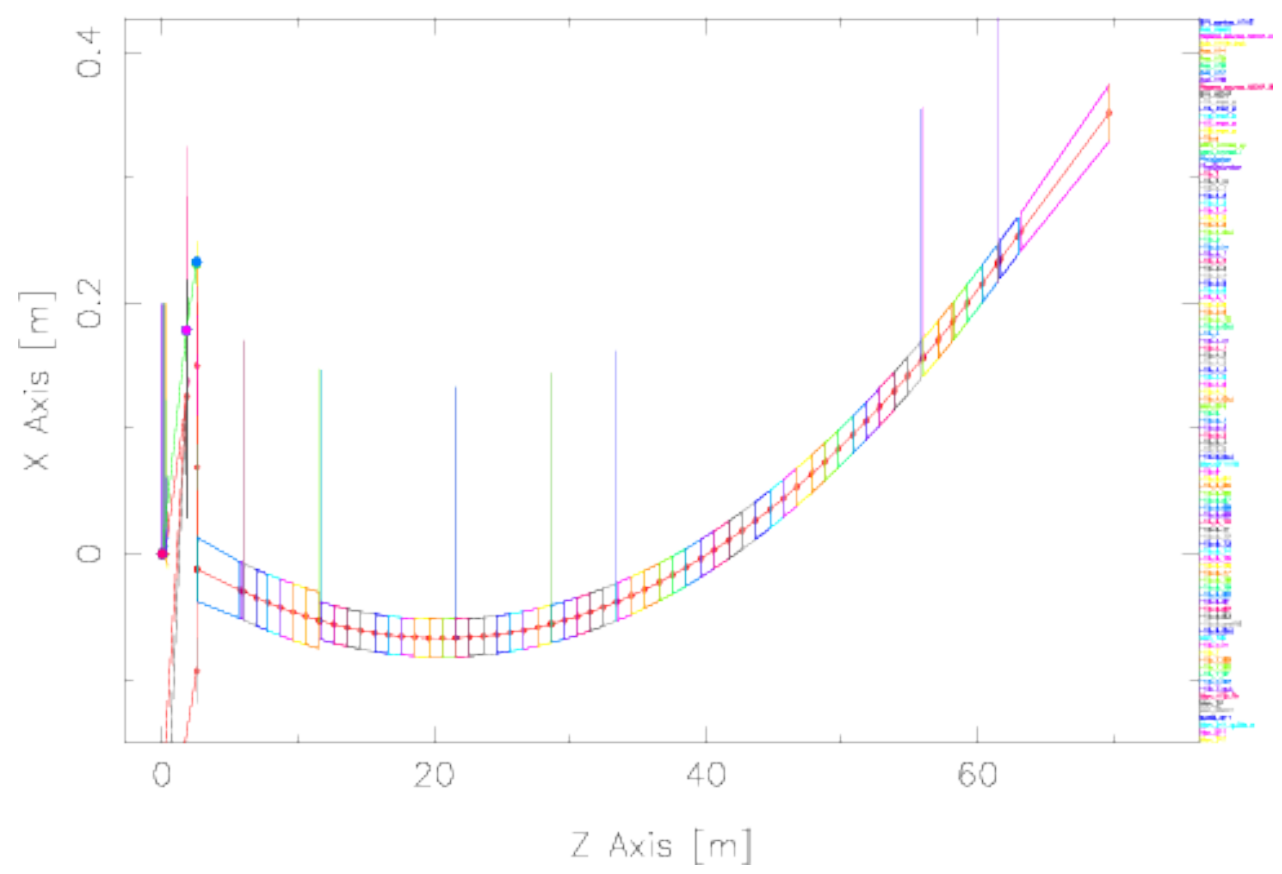

(a) $\mathrm{H} 15$

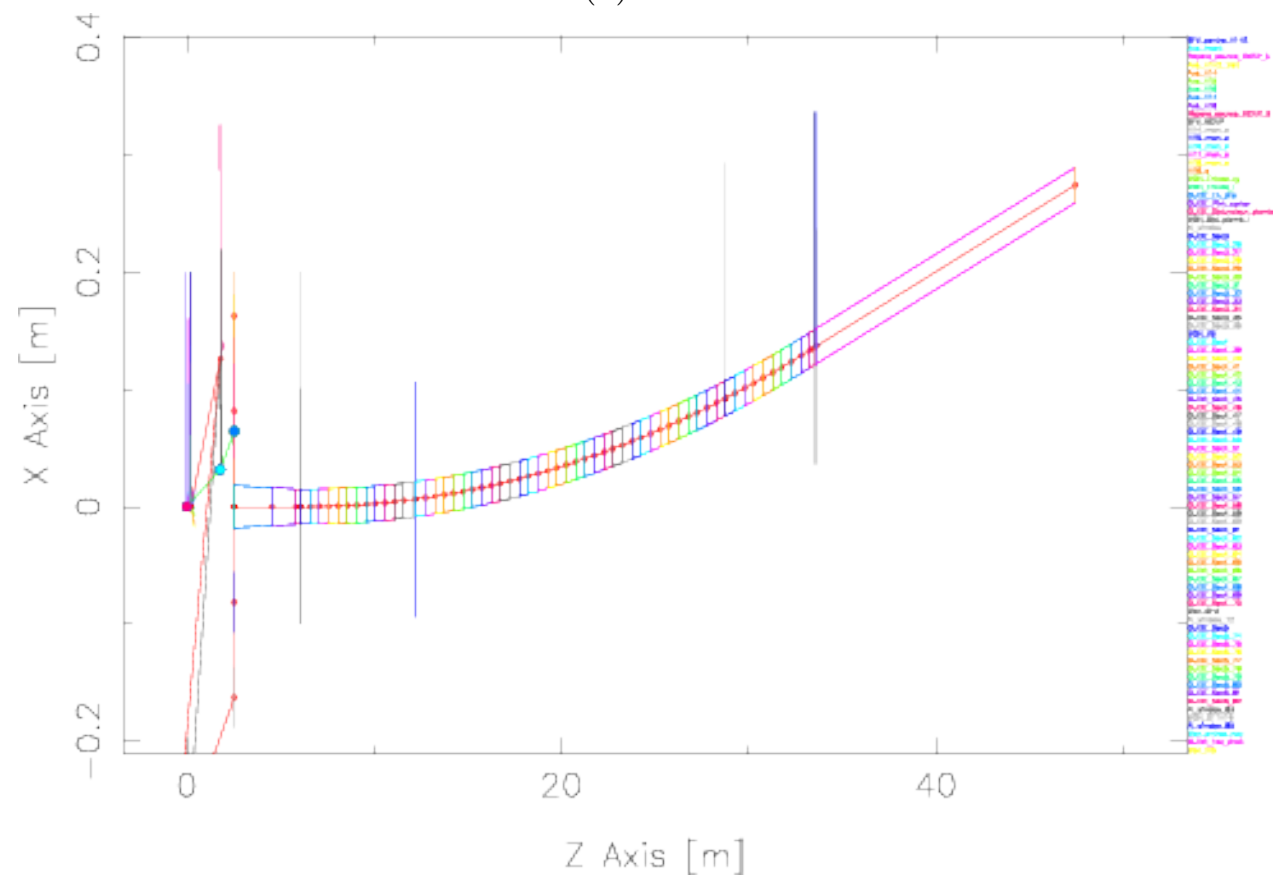

(b) $\mathrm{H} 16$

Figure A.4: Schematic views from above of 2015's H15 and H16, generated by McStas. 


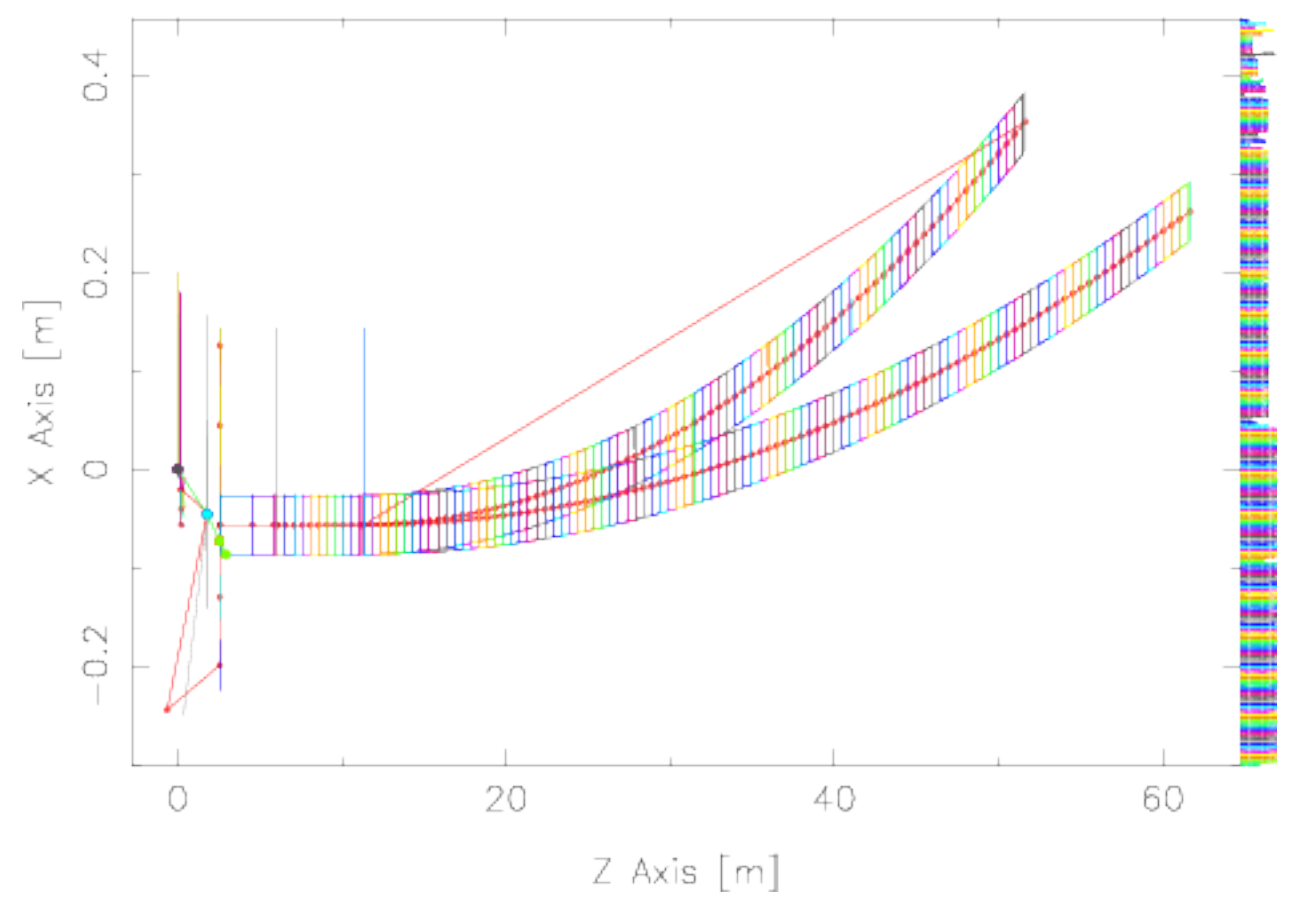

Figure A.5: Schematic views from above of renovated options of H16 generated by McStas.

available instrument position opened at the end of guide. The description of such a future beam is reported in Table A.3. On Fig. A.5, one can see the D7, beam line which is the one subjected to the largest curvature. The curvature for the other guide was set to $4000 \mathrm{~m}$. Figures A.6 a-b display the neutron flux and gain brought forward by this preliminary renewed geometry. One can observe that the refurbishing effort may bring important benefits to the instruments in terms of neutron flux delivered to the entrance, which once integrated over the whole wavelength band gives a gain factor around 2.9. The result should be considered as noticeable, since such a gain only comprises those neutrons useful for experimental measurements. Such a gain mainly results from the increase in the mirror coating that increases the accepted divergence and reduction of characteristic wavelength from $2.77 \AA$ to $2.27 \AA$. However, the smaller height of the guide increases the losses due to the repeated bounces, especially for the long wavelengths. This is largely counterbalanced by the two previous features, as well as by the guide width that is now doubled.

The D11 small-angle scattering instrument will certainly stay at its current position at the end of a $\mathrm{H} 15$ branch. Because it only needs a $\mathrm{m}=0.65$ equivalent divergence, gaining flux for this instrument is solely controlled by the number of reflections from the source to the instrument and by the existence of guide cuts and membranes. Two solutions for the new H15 guide are drawn in Fig. A.7 a-b and the dimensions are in Table A.3. Only the first option has been tested for D11. It consists of a $210 \times 45 \mathrm{~mm}^{2}$ guide, which is horizontally split into three sub-channels, the bottom one being dedicated to D11. From Fig A.8 a-b, one can see that the suggested geometry could increase the flux slightly at the end of the guide. Averaging over the whole wavelength band, the instrument might gain $15 \%$ of flux. Considering that the two renewal options are not drastically different 


\begin{tabular}{|c|c|c|c|c|}
\hline & $\begin{array}{l}\text { Cross-section } \\
\qquad\left(\mathrm{mm}^{2}\right)\end{array}$ & $\begin{array}{l}\text { Length } \\
(\mathrm{m})\end{array}$ & $\begin{array}{l}\text { Curvature radius } \\
\qquad(\mathrm{m})\end{array}$ & $\begin{array}{l}\text { Mirror coating } \\
\qquad(\mathrm{v} \times \mathrm{h})\end{array}$ \\
\hline H15 & $210 \times 45$ & 8.9 & $\infty$ & $\mathrm{m}=3$ \\
\hline \multirow[t]{2}{*}{$1^{\text {st }}$ option } & $210 \rightarrow 250 \times 45$ & 20 & 2000 & $\mathrm{~m}=3$ \\
\hline & \multicolumn{4}{|c|}{ Triple split } \\
\hline \multirow[t]{2}{*}{ IN5 branch } & $\begin{array}{c}150 \times 45 \\
\rightarrow 50 \times 15\end{array}$ & 61 & 2000 & $\begin{array}{c}\mathrm{m}=3 \\
\rightarrow \mathrm{m}>3\end{array}$ \\
\hline & \multicolumn{4}{|c|}{ IN5 } \\
\hline \multirow[t]{2}{*}{ D11 branch } & $50 \times 45$ & 32 & 1000 & $\mathrm{~m}=2 \times 1$ \\
\hline & \multicolumn{4}{|c|}{ D11 velocity selector } \\
\hline H15 & $210 \times 60$ & 8.9 & $\infty$ & $\mathrm{m}=3$ \\
\hline \multirow[t]{2}{*}{$2^{\text {nd }}$ option } & $210 \times 60 \rightarrow 90$ & 18 & $\infty$ & $\mathrm{m}=3$ \\
\hline & \multicolumn{4}{|c|}{ Triple split } \\
\hline \multirow[t]{2}{*}{ IN5 branch } & $\begin{array}{c}150 \times 45 \\
\rightarrow 50 \times 15\end{array}$ & 61 & 1500 & $\begin{array}{c}\mathrm{m}=3 \\
\rightarrow \mathrm{m}>3\end{array}$ \\
\hline & \multicolumn{4}{|c|}{ IN5 } \\
\hline \multirow[t]{2}{*}{ D11 branch } & $50 \times 45$ & 32 & 2800 & $\mathrm{~m}=2 \times 1$ \\
\hline & \multicolumn{4}{|c|}{ D11 velocity selector } \\
\hline \multirow[t]{5}{*}{ H16 } & $210 \times 60$ & 8.9 & $\infty$ & $\mathrm{m}=2 \times 3$ \\
\hline & \multicolumn{4}{|c|}{ Double split } \\
\hline & $100 \rightarrow 120 \times 60$ & 20 & 2000 & $\mathrm{~m}=2 \times 3$ \\
\hline & $120 \times 60$ & 20 & 2000 & $\mathrm{~m}=2$ \\
\hline & \multicolumn{4}{|c|}{ D7 } \\
\hline
\end{tabular}

Table A.3: Schematic description of H15 and H16 renovated preliminary designs. 


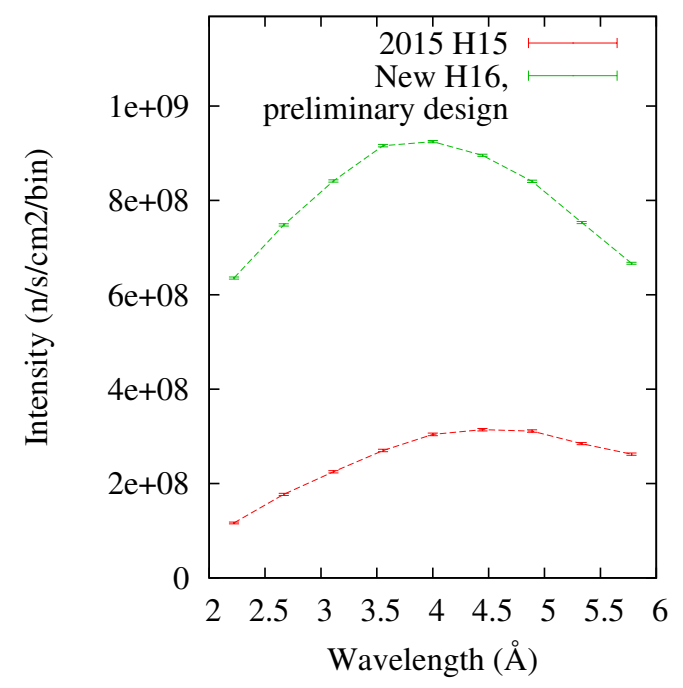

(a) Flux at guide exit as a function of wave length.

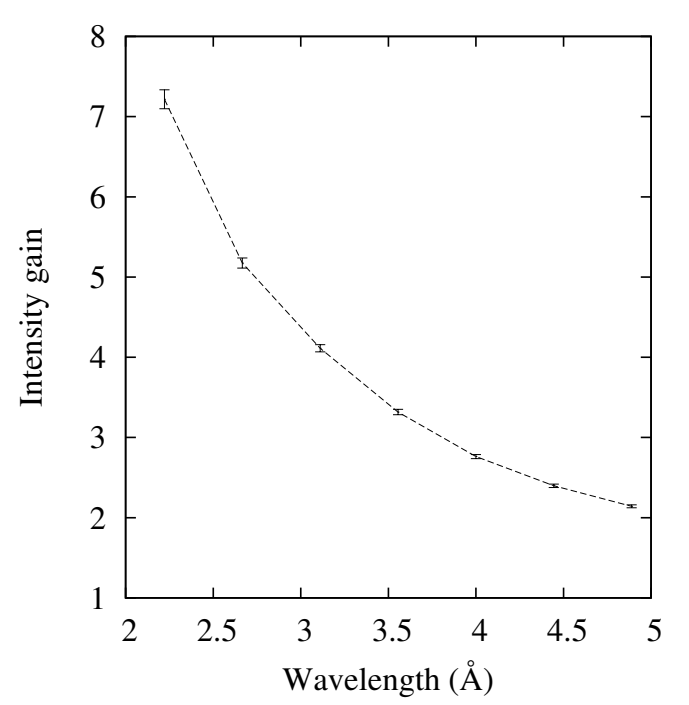

(b) Flux ratio of new design by the current one.

Figure A.6: D7 instrument.

for D11, one can think that the yielded flux of the second solution should be of the same order of magnitude than the one presented here.

As far as the IN5 cold chopper spectrometer is concerned, both renewal options appear appealing since they both comprise an increased $m$ guide coating of 2 . The simulated fluxes and gains are shown in Fig. A.9 a-b. The main difference between them is that the horizontal splitting of the first option allows us to bend the guide before the division, because the horizontal gradient of wavelength and intensity does not impact the beam lines. The second option starts with a $60 \mathrm{~mm}$ wide entrance and grows up to $90 \mathrm{~mm}$. Such an option allows an horizontal splitting of the beam and a natural ballistic shape. However, one cannot bend the guide before the division, since the inner guide would then have less flux than the outer section. The consequence of such a constraint is that the second design philosophy will force a stronger curvature after the split, which will have some impact on the transport of short wavelength neutrons. This is exactly what we can observe in Fig A.9 a-b. Option 1 is more efficient at short wavelengths than the second, while the converse is true for long wavelengths. This latter solution yields a higher gain of 2.2 over the whole spectrum, compared to 1.9 for the first geometry.

These preliminary simulations illustrate the interest to refurbish the old H15-H16 guides. In terms of performance, the cold instruments would benefit from much higher flux that would reduce the measurement time drastically. This study calls for deeper investigations that could use computational parameter optimization and generate geometries that could be counter-intuitive but more efficient in terms of neutron transport. 


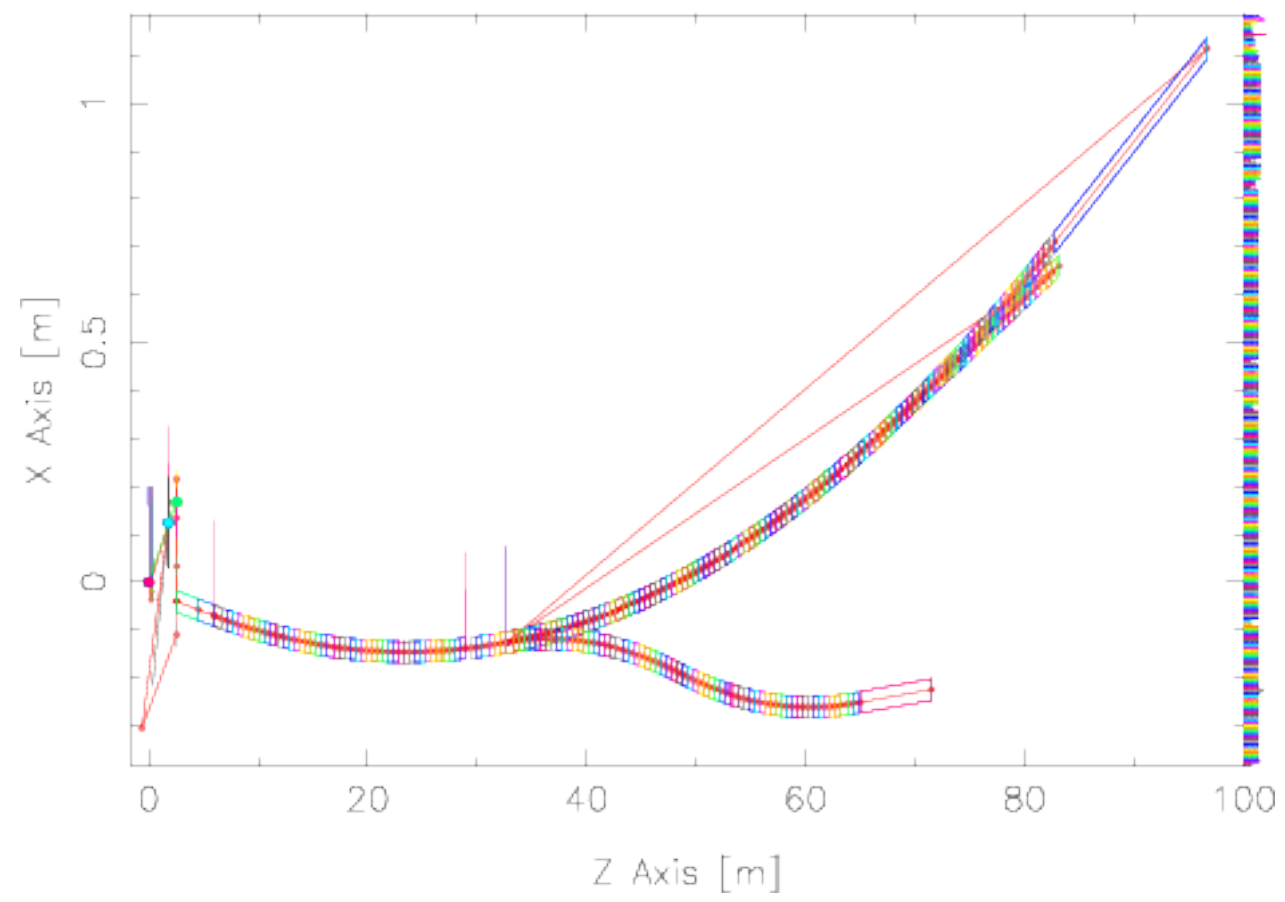

(a) First option with IN5 and D11 top and bottom branches, respectively.

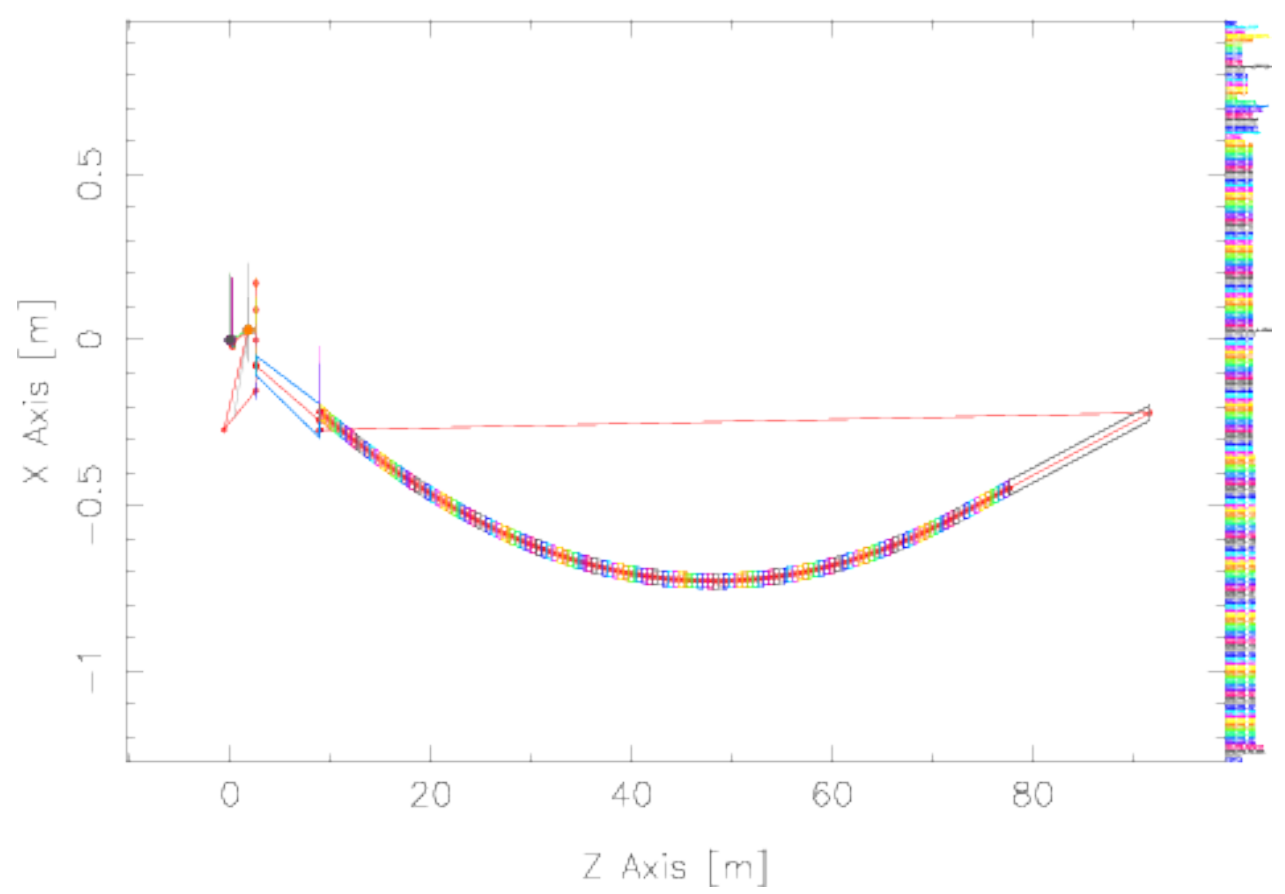

(b) Second option; only the IN5 line can be seen.

Figure A.7: Schematic views from above of first and second renovated options of H15, generated by McStas. 


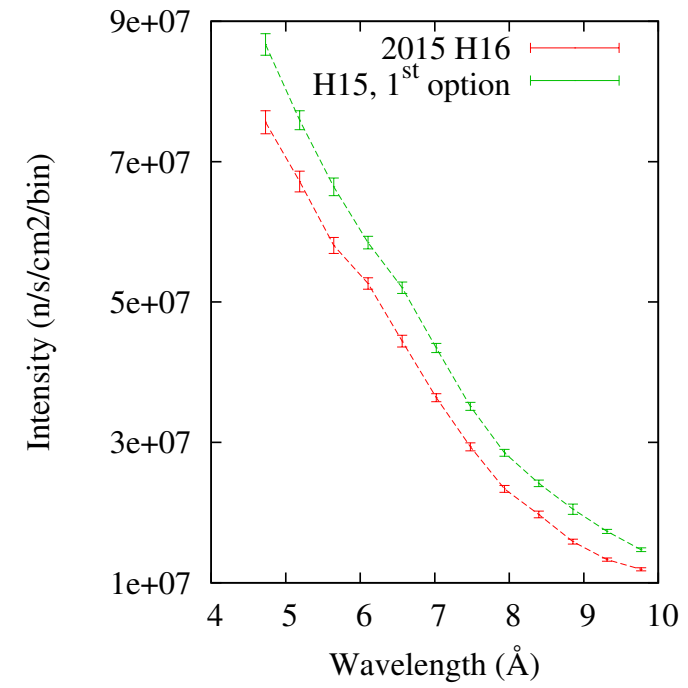

(a) Flux at guide exit as a function of wave length.

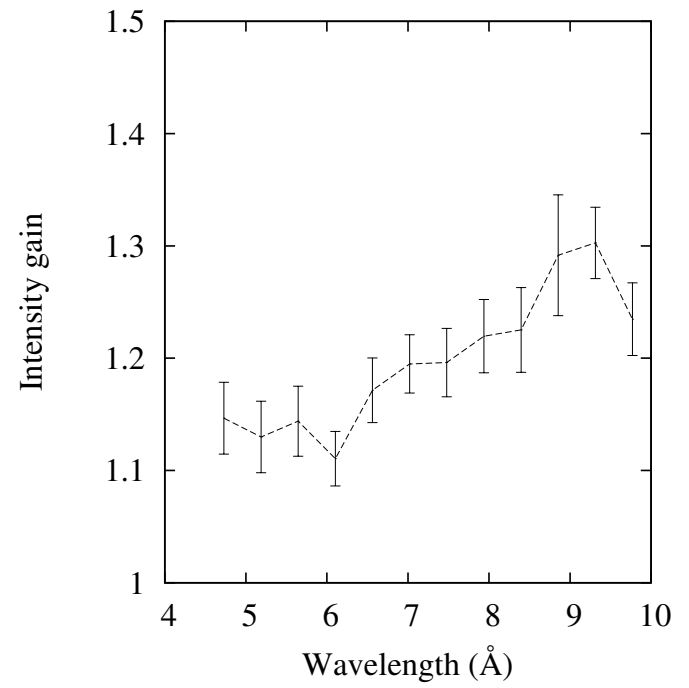

(b) Flux ratio of new design by the current one.

Figure A.8: D11 instrument.

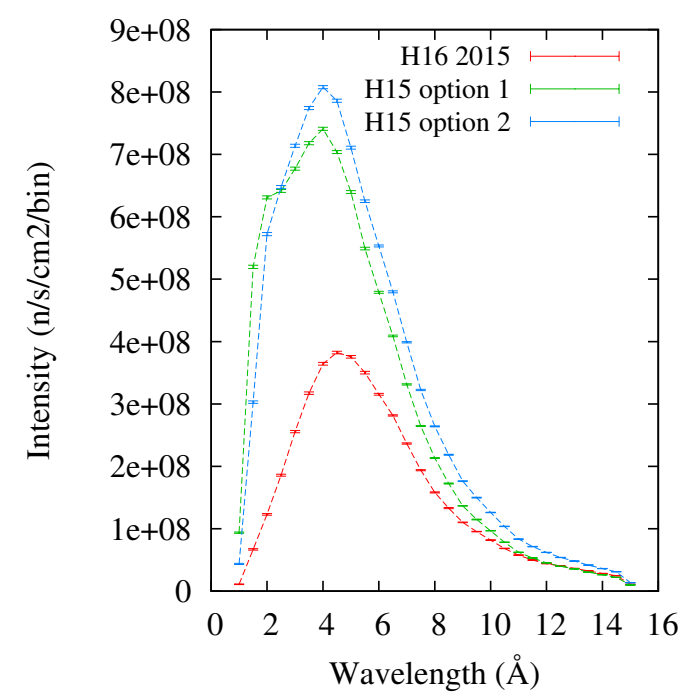

(a) Flux at guide exit as a function of wave length.

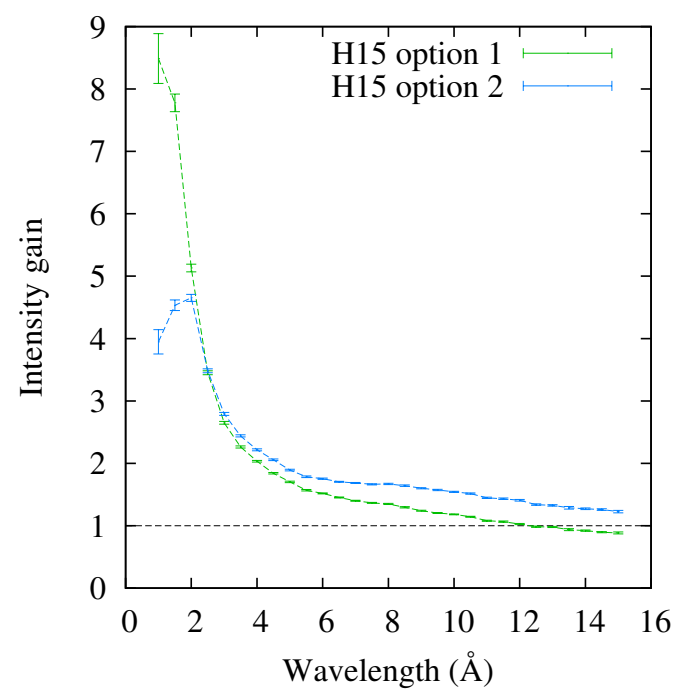

(b) Flux ratio of new design by the current one.

Figure A.9: IN5 instrument. 


\section{A.3 $\quad \mathrm{H} 24$}

The complete refurbishment of this guide aims at replacing $\mathrm{H} 23$ and $\mathrm{H} 24$, which are currently installed. At this time, H23 provides neutrons to CT1 and T13 A-C technical instruments. H24 transports neutrons to more instruments, such as IN3, IN13, D10, CT2, Cyclops, and Orient Express. The current geometry of H24, though rather simple, is reported in Table A.4. Instruments' monochromators are installed on the main beam which provokes a guide cut, and losses, for the downstream instruments. Renovating this guide thanks to super-mirror components will allow us to create more beam lines from a single source and, consequently, have more dedicated beam lines to host new instruments. Considering the in-pile part, the entrance of the guide will be located at the same position as H24. Its geometry, also written in Table A.4, consists of a $210 \times 90 \mathrm{~mm}^{2}$ guide that will widen to $98 \mathrm{~mm}$ and then split into three sub channels: H241, H242, and H243. The widening length had to be optimised, and this study is described in the next section. H241 should be dedicated to Xtreme-D, an upgrade of the IN3 three-axis spectrometer; H242 should bring neutrons to IN13, D10, and technical instruments; and a last beam, H243, will go to Aladin. The performances of this guide will be calculated for Xtreme-D, IN13, and D10, because these are the major instruments of the project. Schematic views from present and preliminary refurbished H24 can be gauged from Fig A.10 a-b.

\section{A.3.1 Diverging section}

The need of a guide with a widening section stems from the lack of space within the in-pile part to directly install a $98 \mathrm{~mm}$ wide guide. Such extra width will allow us to vertically split the guide into $45 \mathrm{~mm}$ sub-channels. Therefore, it was decided to start with a $90 \mathrm{~mm}$ wide beam until the "safety valve", and then to expand it to $98 \mathrm{~mm}$. The "safety valve" is a piece of equipment made to shut-off the beam; it consists of a hole in the pool wall followed by a neutron absorbing element that can be placed or removed from on the beam path. As the hole dimension cannot be modified, the guide passing through it must fulfil some requirements, such as a maximum width around $90 \mathrm{~mm}$. An increase to $98 \mathrm{~mm}$ may lead to a decrease in flux. For a step-shaped transition, the flow conservation dictates that the intensity will be divided by a factor $d_{w}$,

$$
\begin{gathered}
d_{w}=\frac{\text { Original flux }}{\text { Diluted flux }}=\frac{\text { Downstream width }}{\text { Upstream width }} \\
\text { in our case : } \quad d_{w}=\frac{98}{90}=1.089
\end{gathered}
$$

The solution adopted to compensate for the aforementioned flux decrease involves a conversion of the beam divergence, which is achievable using neutron optic tools. That is, if the beam downstream to the widening section has a mirror with $\mathrm{m}=2$, one has to put a higher $\mathrm{m}$ mirror upstream and convert the neutrons with a divergence above $\mathrm{m}=2$ to one below in order to have a guide following the specification. This is done by reflection on inclined mirrors. Figure A.11 shows the reduction of the divergence of a neutron trajectory.

The next task was to find out what the optimal length of the diverging guide should 


\begin{tabular}{|c|c|c|c|c|}
\hline & $\begin{array}{l}\text { Cross-section } \\
\left(\mathrm{mm}^{2}\right)\end{array}$ & $\begin{array}{l}\text { Length } \\
(\mathrm{m})\end{array}$ & $\begin{array}{l}\text { Curvature radius } \\
\qquad(\mathrm{m})\end{array}$ & $\begin{array}{l}\text { Mirror coating } \\
\qquad(\mathrm{v} \times \mathrm{h})\end{array}$ \\
\hline \multirow[t]{8}{*}{ H24 2015} & $200 \times 45$ & 2.7 & $\infty$ & $\mathrm{m}=2$ \\
\hline & $200 \times 45$ & 5.5 & 11850 & $\mathrm{~m}=2$ \\
\hline & $200 \times 30$ & 67.75 & 11850 & $\mathrm{~m}=1$ \\
\hline & \multicolumn{4}{|c|}{ IN3 } \\
\hline & $125 \times 30$ & 6.25 & $\infty$ & $\mathrm{m}=1$ \\
\hline & \multicolumn{4}{|c|}{ IN13 } \\
\hline & $125 \times 30$ & 13.3 & $\infty$ & $\mathrm{m}=1$ \\
\hline & \multicolumn{4}{|c|}{ D10 } \\
\hline \multirow[t]{3}{*}{ H24 renovated } & $210 \times 90$ & 11.4 & $\infty$ & $\mathrm{m}=3$ \\
\hline & $210 \times 90 \rightarrow 98$ & $L$ & $\infty$ & $\mathrm{m}=3$ \\
\hline & \multicolumn{4}{|c|}{ Triple split } \\
\hline \multirow[t]{2}{*}{ Xtreme-D branch } & $210 \times 45$ & $75-L$ & 12000 & $\mathrm{~m}=2$ \\
\hline & \multicolumn{4}{|c|}{ Xtreme-D } \\
\hline IN13 and D10 & $125 \times 45$ & $81.3-L$ & 20000 & $\mathrm{~m}=2$ \\
\hline \multirow[t]{3}{*}{ branch } & \multicolumn{4}{|c|}{ IN13 } \\
\hline & $125 \times 45$ & 13.5 & 20000 & $\mathrm{~m}=2$ \\
\hline & \multicolumn{4}{|c|}{ D10 } \\
\hline
\end{tabular}

Table A.4: Schematic descriptions of H24, as installed in 2015, and the renovated geometry studied. 


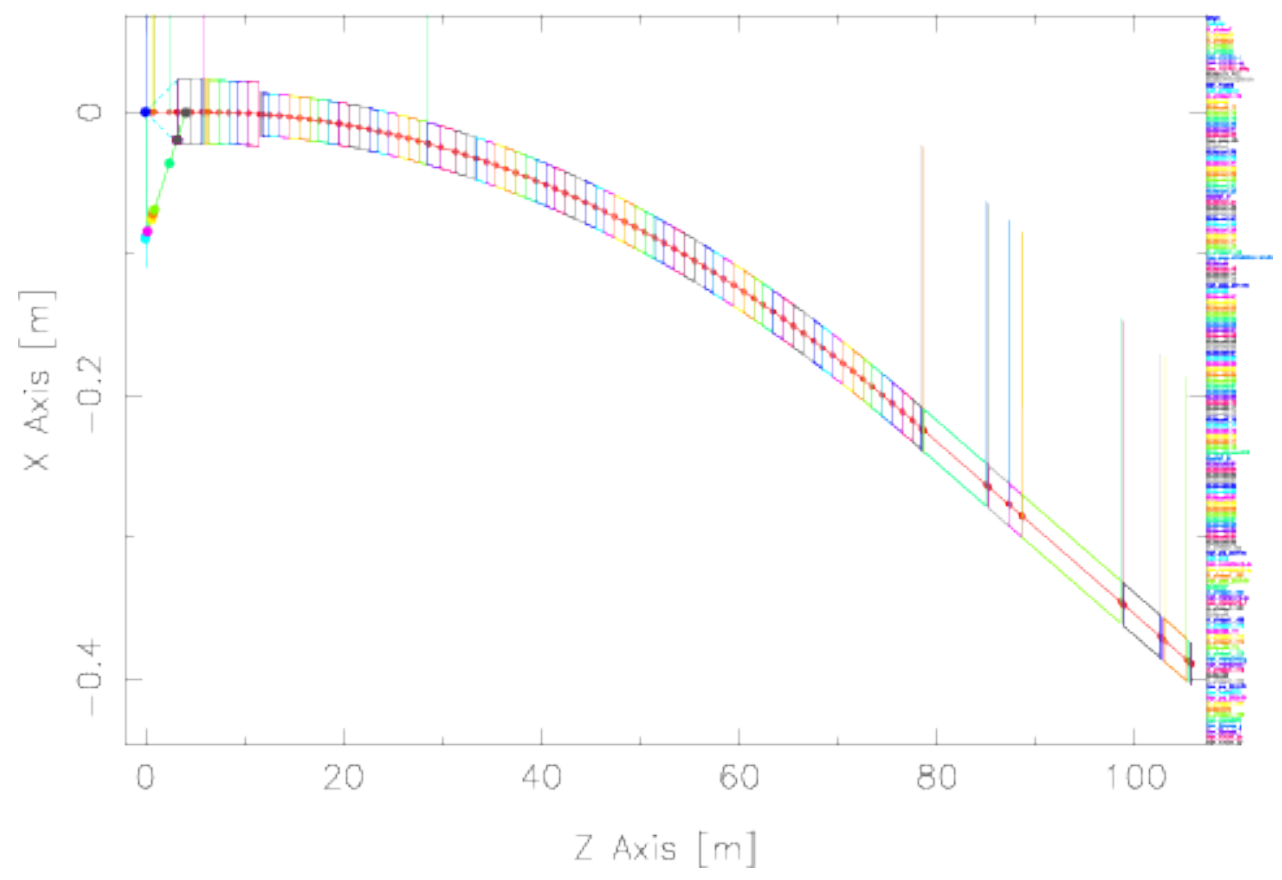

(a) 2015's H24

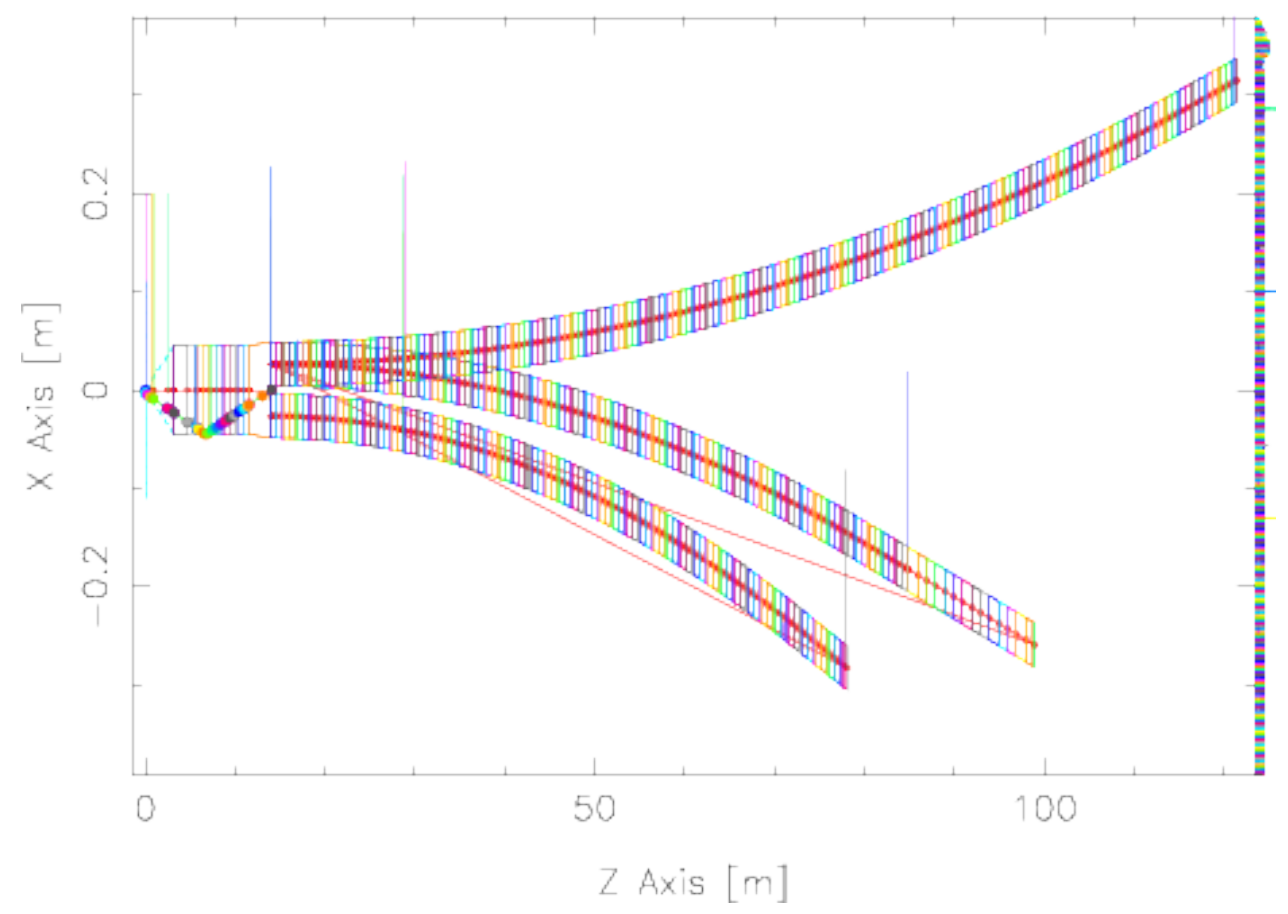

(b) Renovated H24, preliminary design

Figure A.10: Schematic views from above of H24, generated by McStas. The colored dots are the tracking of a neutron trajectory all along a beam line. 


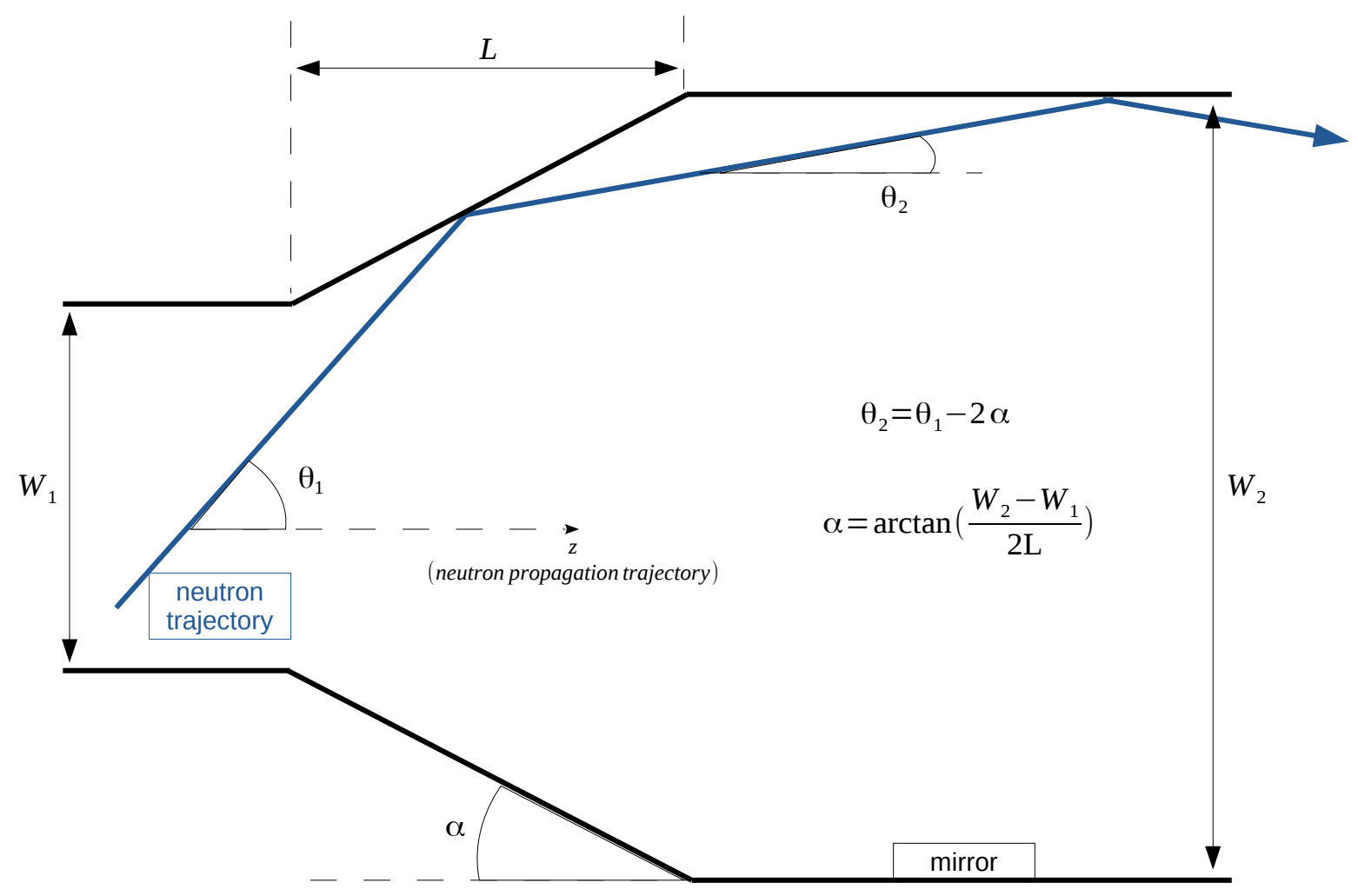

Figure A.11: Reduction of the divergence of a given neutron using a linear transition.

be. Notice that we only consider the case of single linear growth, and therefore left other, more sophisticated alternatives, such as parabolic geometries, for further study. First,we could analytically find what the maximum and minimum values, for which we took the following steps:

Maximum length. This can be found by considering the case in which the neutrons that are not reflected on the diverging mirror have a divergence equal or below " $m=2 "$. This gives :

$$
L_{\max }=\frac{W_{1}+W_{2}}{2 \times \tan \left(\gamma_{c \text { at } m=2}\right)}
$$

with $\gamma_{c \text { at } m=2}$ as the critical angle for $\mathrm{m}=2$ coating. As an approximation, we considered only the neutrons at $1.8 \AA$ due to the fact that it is the peak of intensity of the thermal source. The numerical application gave

$$
L_{\max }=15.1 \mathrm{~m}
$$

Minimal length. This is computed from the limit at which the particles with the highest divergence cannot be reflected on the diverging part :

$$
L_{\text {min }}=\frac{W_{2}-W_{1}}{2 \times \tan \left(\gamma_{c \text { at } m=3}\right)}=0.43 \mathrm{~m}
$$

We proceeded to get an estimation of an optimal length within the limits given above. 
For such a purpose, one can consider the opening angle necessary to reduce $\mathrm{m}=3$ divergence into a $\mathrm{m}=2$ in only one bounce. Indeed, in order to have two bounces, it would need around $L=\frac{W_{2}+W_{1}}{2 \times \tan \left(\gamma_{c} \text { at } m=2.5\right)}=11 \mathrm{~m}$, which is not possible as the maximum length technically available for the diverging element is $4.5 \mathrm{~m}$. The pertinent equation is:

$$
\begin{gathered}
\gamma_{1}-2 \times \alpha=\gamma_{2} \\
\Leftrightarrow 1.73 \cdot 10^{-3} \times m_{1} \times \lambda-2 \cdot \tan \left(\frac{W_{2}-W_{1}}{2 L_{\text {opt. }}}\right)=1.73 \cdot 10^{-3} \times m_{2} \times \lambda \\
\Leftrightarrow L_{\text {opt. }}=\frac{\Delta W}{2 \cdot \tan \left(\frac{1.73 \cdot 10^{-3} \times \lambda \times \Delta m}{2}\right)}
\end{gathered}
$$

Considering the very low reflection angles of the neutrons, that can be approximated by

$$
L_{\text {opt. approx. }}=\frac{\Delta W}{1.73 \cdot 10^{-3} \times \lambda \times \Delta m}
$$

with $\Delta m$ being the $m$ difference between the upstream and downstream guide, and $\Delta W$ the width difference between the upstream and downstream guide. The numerical application for neutrons at $1.8 \AA$ gives $L_{\text {opt }}=2.57 \mathrm{~m}$. This value had to be cross-checked by simulation in order to know if it was valid for a true thermal spectrum. The method used was to implement a monitor before and after the diverging element and count only the neutrons which have a divergence equal to or below $\mathrm{m}=2$. A ratio of these flux will give us the flux decrease factor as a function of the length of the element. In doing this, we do not take into account the neutrons absorbed in the element by the mirrors, a contribution which may be neglected due to the fact that only one bounce is made. Figure A.12 shows the dilution factor as a function of the length of the diverging guide. We can see that it validates the analytical calculation. At around $2.5 \mathrm{~m}$, the flux dilution factor reaches a minimum and then starts to increase slowly. For technical reasons, the exact value chosen will be $2.5 \mathrm{~m}$.

\section{A.3.2 Performance of instruments}

Table A.5 sums up the characteristics of the needed neutron beam for H24 instruments. The knowledge of these values influences the guide optimization process. If one looks at the divergence accepted by the instruments, one can see that only Xtreme-D could use the full divergence of a $\mathrm{m}=2$ guide. For the other, an $\mathrm{m}=1.5$ guide would suffice.

To have a realistic idea of the performance gain thanks to the guide renovation, the beam monitors in McStas simulation took into account the instruments' acceptance in wavelength and divergence. Results from simulation and analytical calculation, are reported in Table A.6 and in Fig. A.13, A.14, and A.15 for Xtreme-D, IN13, and D10, respectively. One can see that the preliminary design bring very interesting gains in intensity for the instruments. Such values can certainly be increased even more thanks to a fine optimisation of the guide parameters. The analytical estimations are always below but yet in good agreement, with the simulation. This trend can be explained by the fact that the estimation does not take into account the benefits from the suppression of guide cuts. The main reason behind the increase of neutron flux intensity is the increase of the 


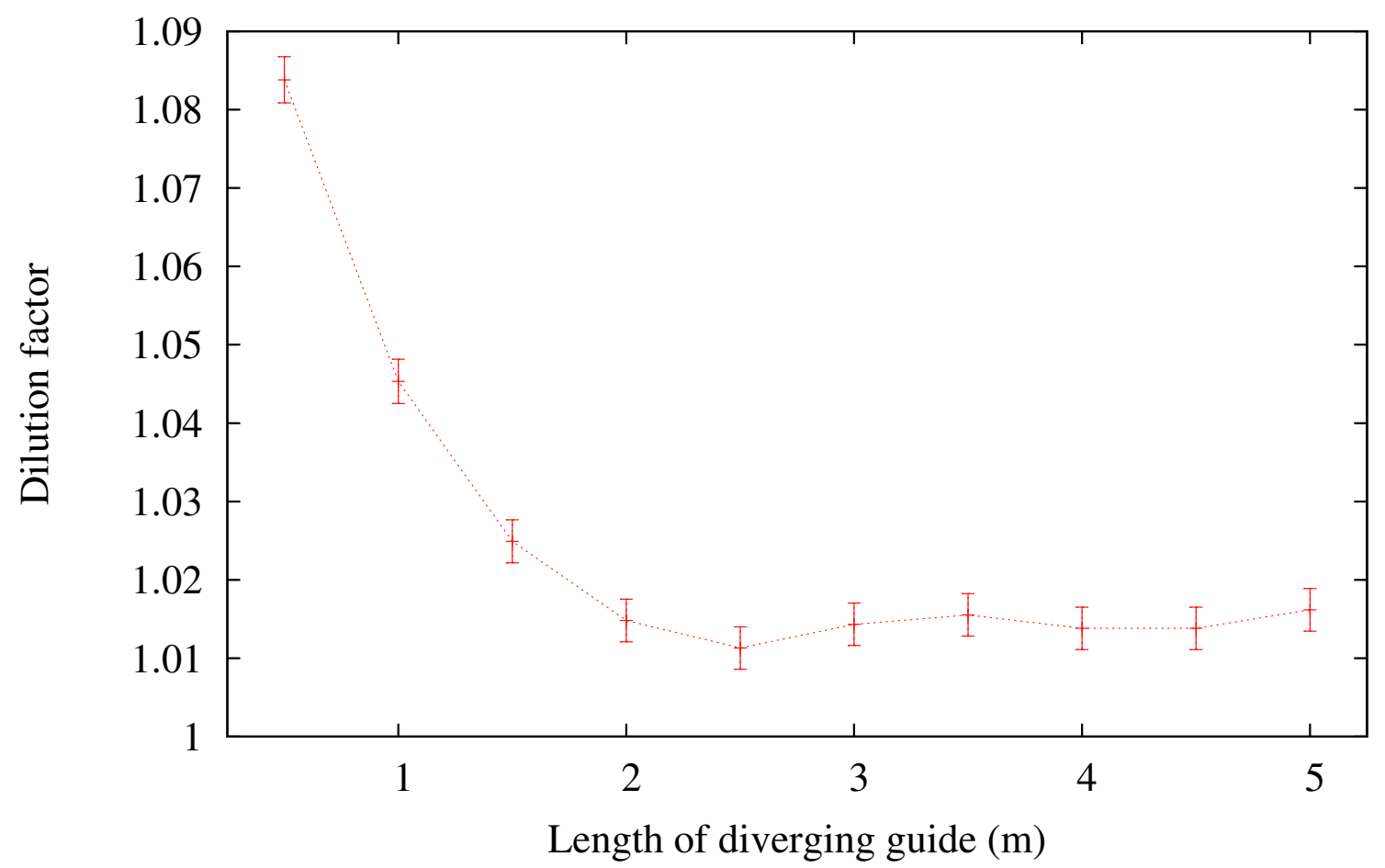

Figure A.12: Dilution factor as a function of the length of the diverging guide, McStas simulation, $m=2$ guide, growth from $90 \mathrm{~mm}$ to $98 \mathrm{~mm}$.

\begin{tabular}{cccc}
\hline & Xtreme-D & IN13 & D10 \\
\hline Wavelength $(\AA)$ & {$[0.8 ; 4.1]$} & {$[2 ; 2.3]$} & {$[1.1 ; 6]$} \\
optimal & {$[1.26 ; 2.36]$} & 2.23 & 1.26 and 2.36 \\
\hline Divergence & $\pm 0.6^{\circ}$ at $2.5 \AA$ & $\pm 0.3^{\circ}$ at $2.23 \AA$ & $\pm 0.3^{\circ}$ at $2.4 \AA$ \\
m-equivalence & 2.5 & 1.35 & 1.25 \\
\hline Beam cross-section & $\geq 45 x 100$ & $\leq 45 x 125$ & $\leq 30 x 125$ \\
(width x height) & & & \\
\hline
\end{tabular}

Table A.5: Neutron beam characteristics for future H24 intruments. 


\begin{tabular}{cccc}
\hline & Xtreme-D & IN13 & D10 \\
\hline$\phi_{\text {actual }}$ & $2.8 \times 10^{8}$ & $6.6 \times 10^{7}$ & $2.7 \times 10^{8}$ \\
$\phi_{\text {future }}$ & $1.1 \times 10^{9}$ & $1.3 \times 10^{8}$ & $5.0 \times 10^{8}$ \\
\hline Gain $_{\text {McStas }}$ & 4.0 & 1.9 & 1.8 \\
Gain $_{\text {analytic }}$ & 3.4 & 1.5 & 1.4 \\
$\mathrm{k}_{\mathrm{m}}$ & 4.0 & 1.8 & 1.6 \\
$\mathrm{k}_{\mathrm{f}}$ & 1.1 & 1.1 & 1.1 \\
$\mathrm{k}_{\mathrm{r}}$ & 0.77 & 0.77 & 0.79 \\
\hline
\end{tabular}

Table A.6: Flux intensity and gain for the preliminary design of refurbished H24. Wavelength in $\AA$ and flux in $\mathrm{n} / \mathrm{cm}^{2} / \mathrm{s}$, simulation statistical uncertainty below $1 \%$.

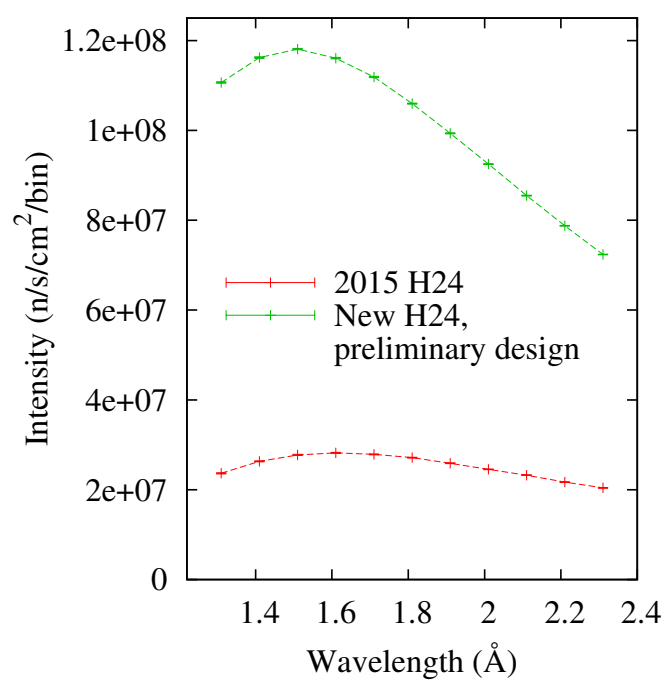

(a) Flux at guide exit as a function of wave length.

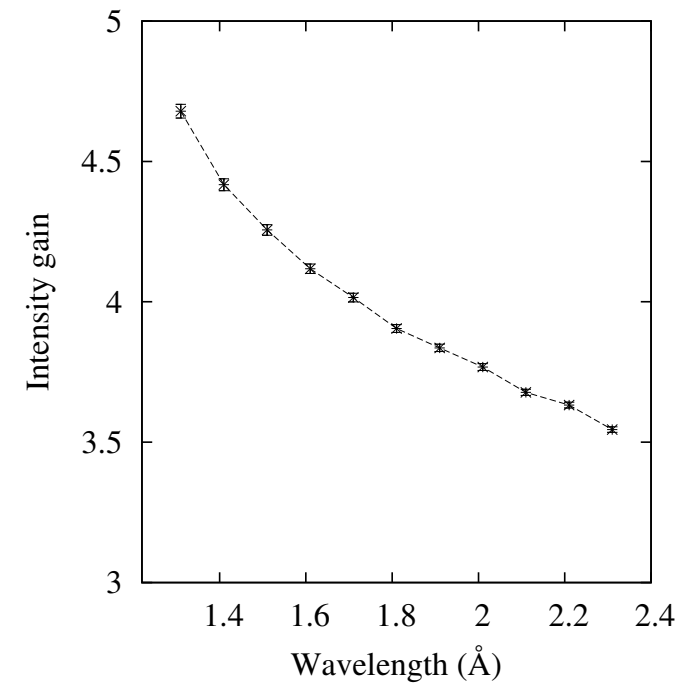

(b) Flux ratio of new design by the current one.

Figure A.13: Xtreme-D instrument.

$\mathrm{m}$-value of the mirrors. It permits us to collect neutrons at higher than $\mathrm{m}=1$ divergence that can be used by the experiments. Also, the filling of the neutron guide is improved.

As for the cold guides, this preliminary study proves the interest to develop innovative guide geometries, taking the full advantage of the super-mirror technology for thermal neutron delivery systems. 


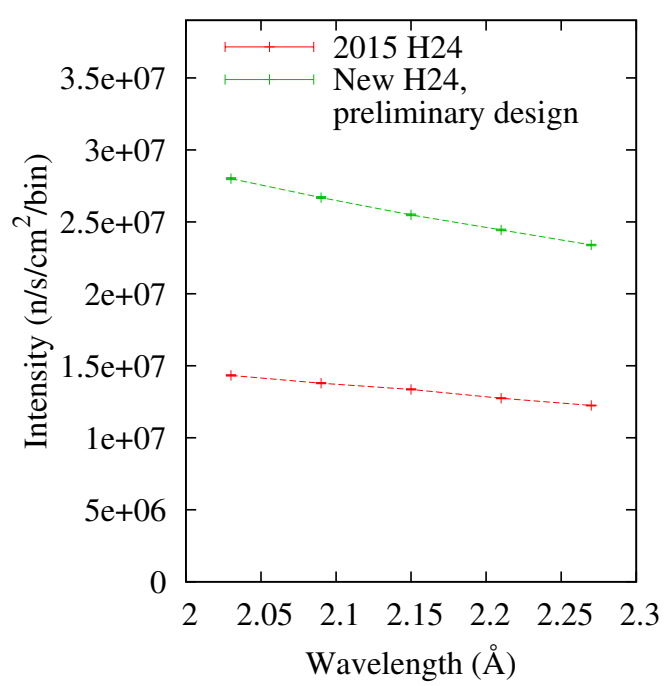

(a) Flux at guide exit as a function of wave length.

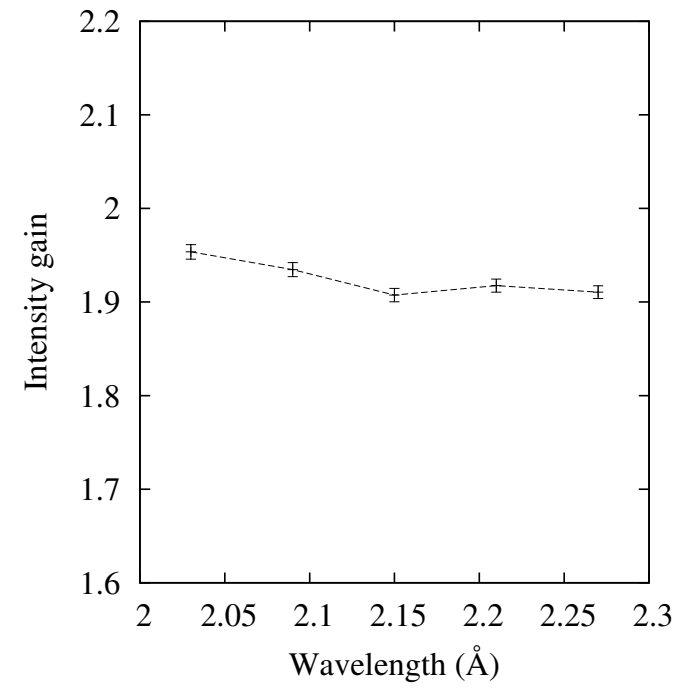

(b) Flux ratio of new design by the current one.

Figure A.14: IN13 instrument.

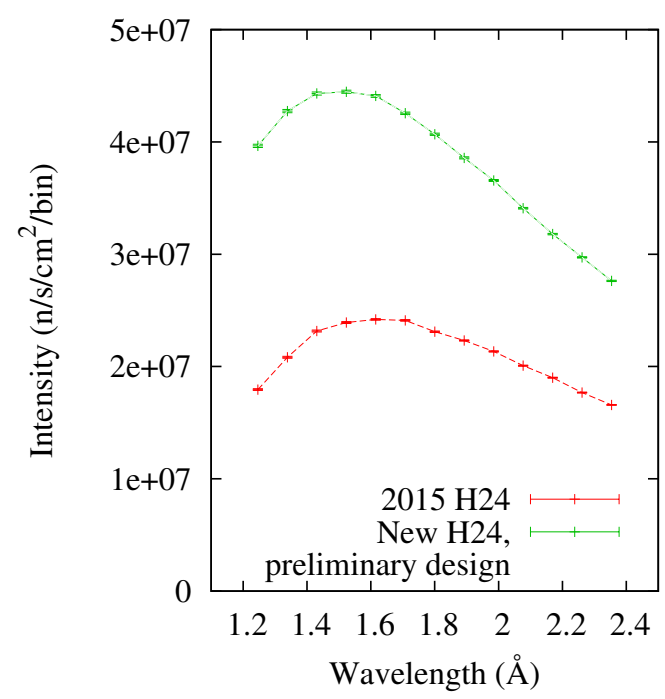

(a) Flux at guide exit as a function of wave length.

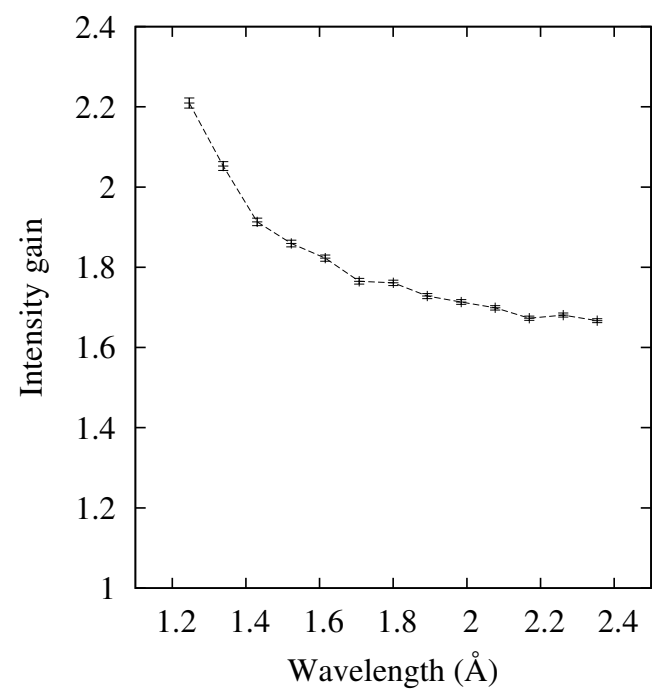

(b) Flux ratio of new design by the current one.

Figure A.15: D10 instrument. 


\section{Appendix B}

\section{Presentation of some examples of the written computation code employed}

The purpose of the present appendix is to provide a taste of the computational effort involved within this Ph.D. thesis. In other words, these are only meant to be short, concise examples of the use of several different neutron propagation and transport packages.

\section{McStas: detection of neutrons penetrating through guide walls}

The following input lines of code are meant to be used within the McStas simulation package and are written to allow the detection of neutrons which are not reflected by a mirror and hence penetrate within the glass substrate. They describe a guide element made of four mirrors (Mirror (...)); one of them does not suppress the non-reflected neutrons (transmit $=1$ ). A large monitor is located after the guide to detect these neutrons (Pertes_s_1_mugd). The particles transmitted through the guide are suppressed thanks to a beam stop element (Beamstop). Neutrons were detected if their wavelength was between $0.875 \AA$ and $15.125 \AA$, and the cosine of their incidence angle from -0.0005 to 0.0505 . The number of bins for each range was 57 and 51, respectively. The final output was a matrix giving the flux for a given wavelength and cosine.

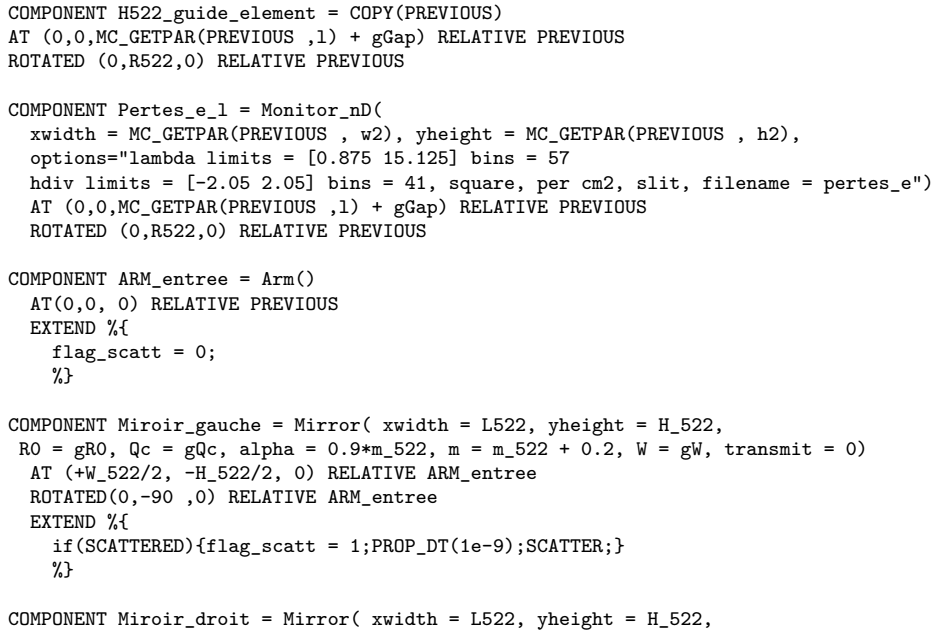




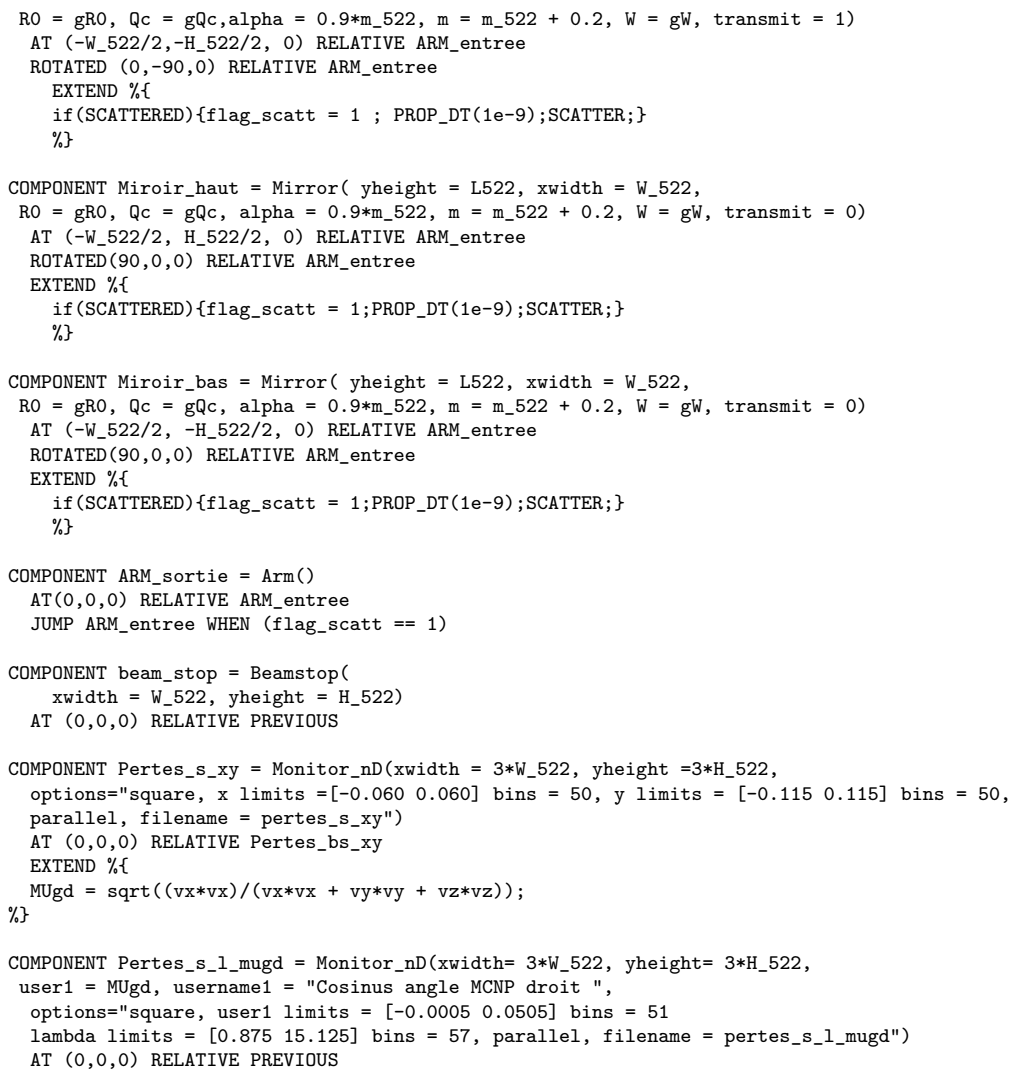

\section{MCNP: propagation of neutrons within the mirror substrate}

In order to compute the ${ }^{10} \mathrm{~B}(\mathrm{n}, \alpha){ }^{7} \mathrm{Li}$ reaction rate in guide reflective walls, a generic glass plate was modelled using MCNP with a neutron source above one of its surfaces. Neutrons were shot at fixed energy and incidence angles to the glass surface. The $(\mathrm{n}, \alpha)$ reactions were then monitored at different depths. This calculation was performed $51 \times 57$ times, with variations of neutron energy and initial trajectory.

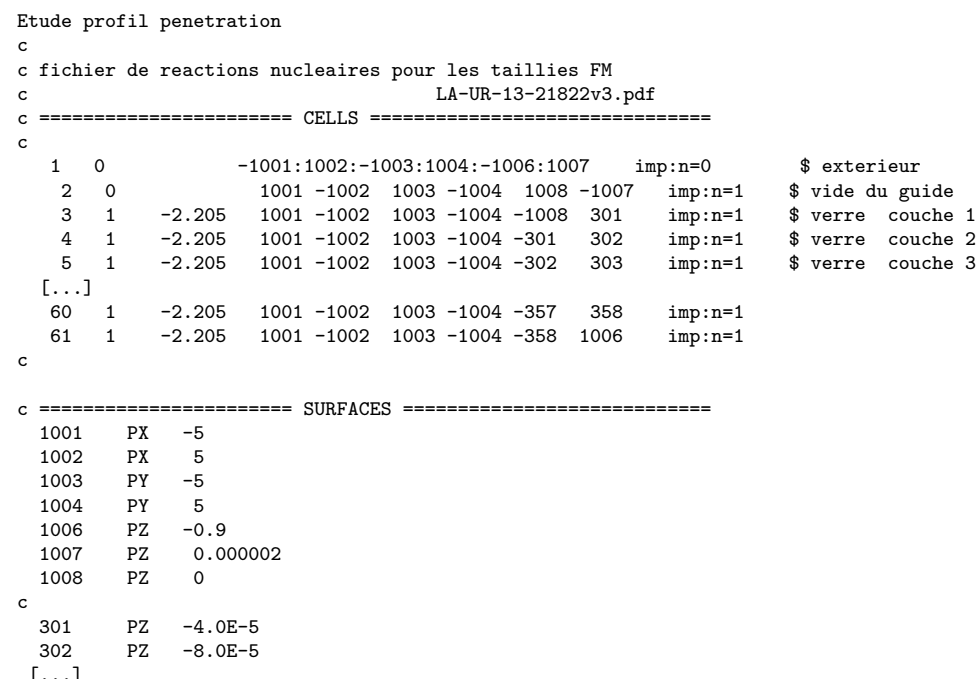




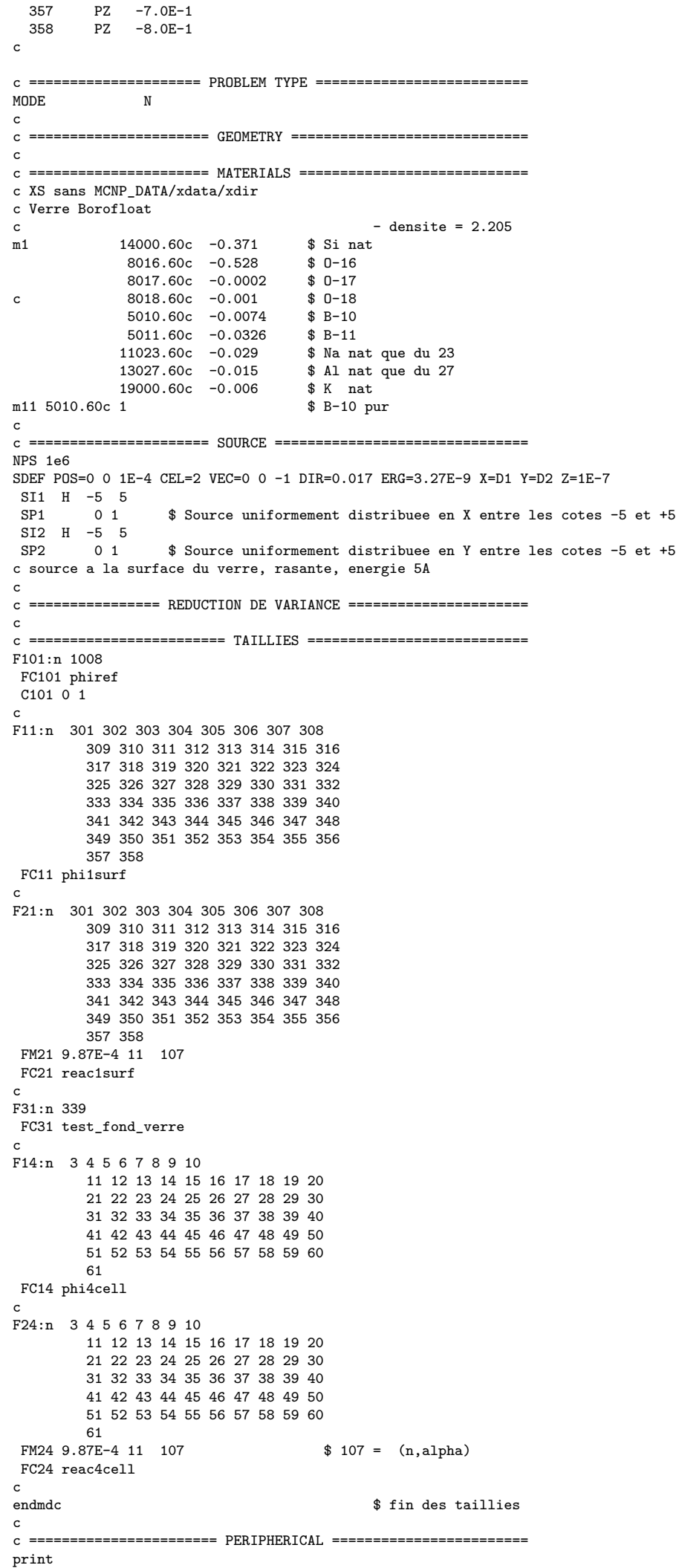

$\$$ fin des taillies 


\section{Awk Unix shell command: coupling the results from McStas and MCNP}

To combine the two previous calculations described above, the MCNP reaction rate had to first be extracted for the whole output file.

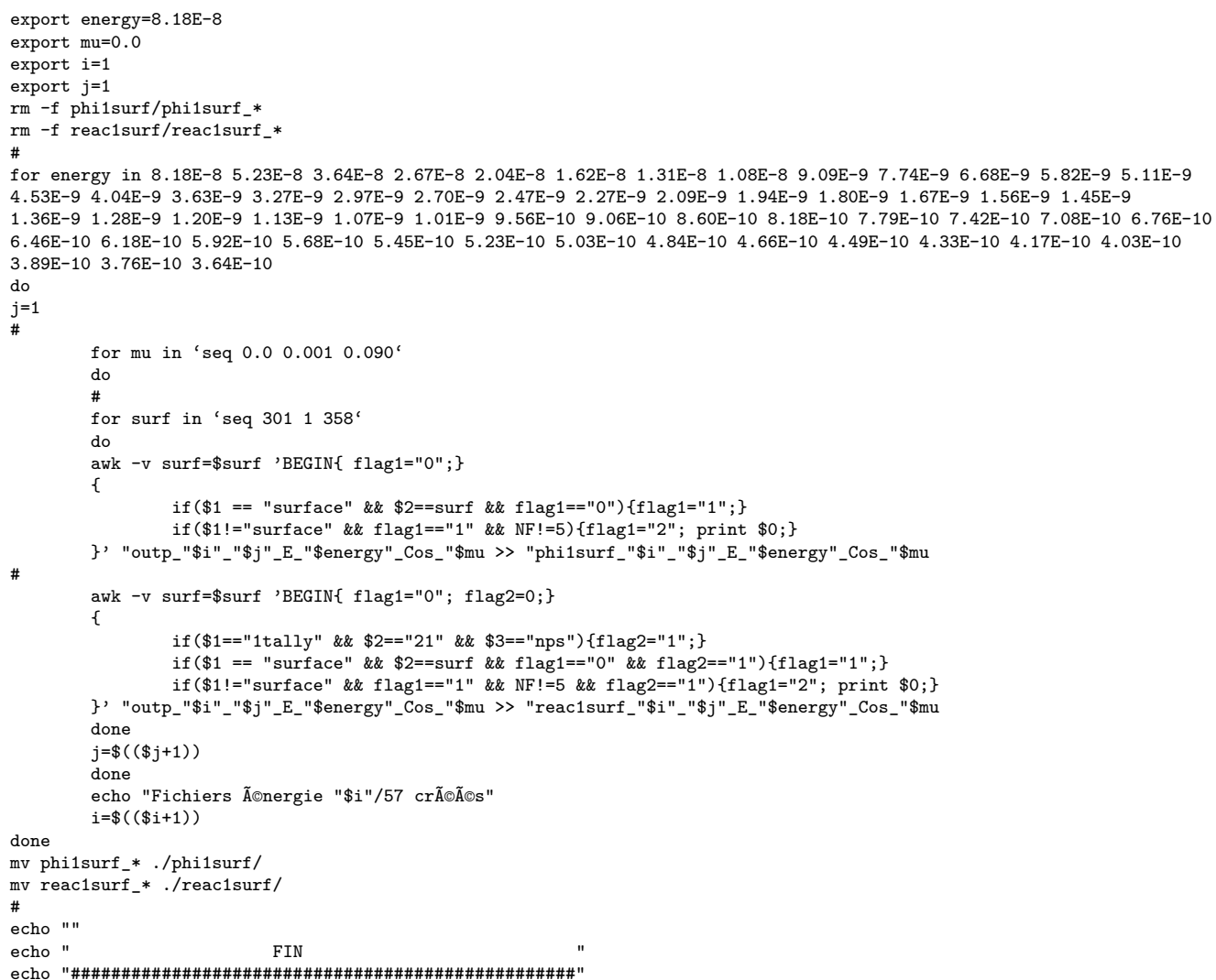

Then, each MCNP reaction rate had to be multiplied by the McStas simulated incident neutron flux to have the real number of $(n, \alpha)$ reactions per cubic centimetre and second. This step of the data treatment gave the reaction rate value reacXfluxSurf_... as well as the uncertainty reacXfluxSurf_erreur_...

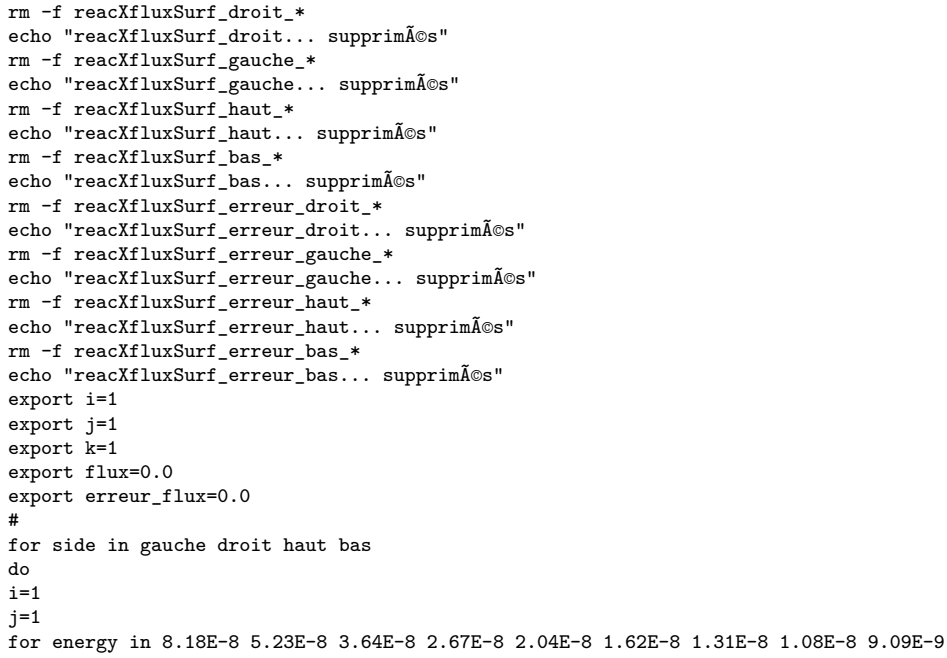




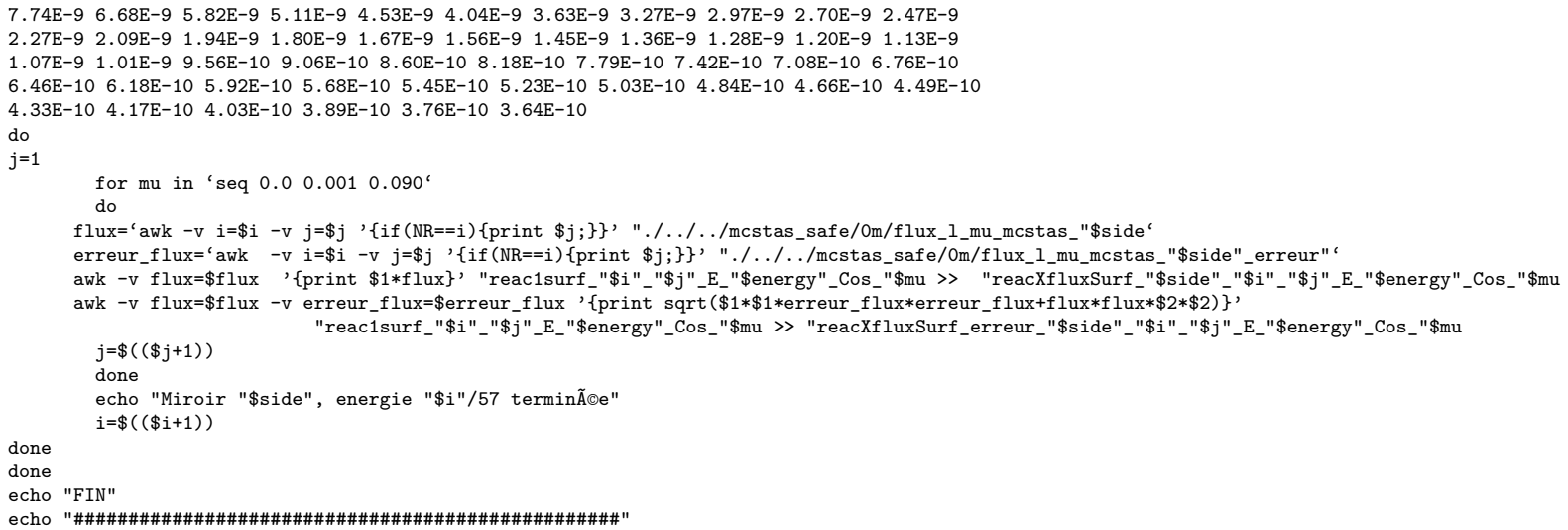

Finally, all the files were merged into one, giving the $(\mathrm{n}, \alpha)$ reaction rate in $/ \mathrm{cm}^{3} / \mathrm{s}$ as a function of the depth from the irradiated surface.

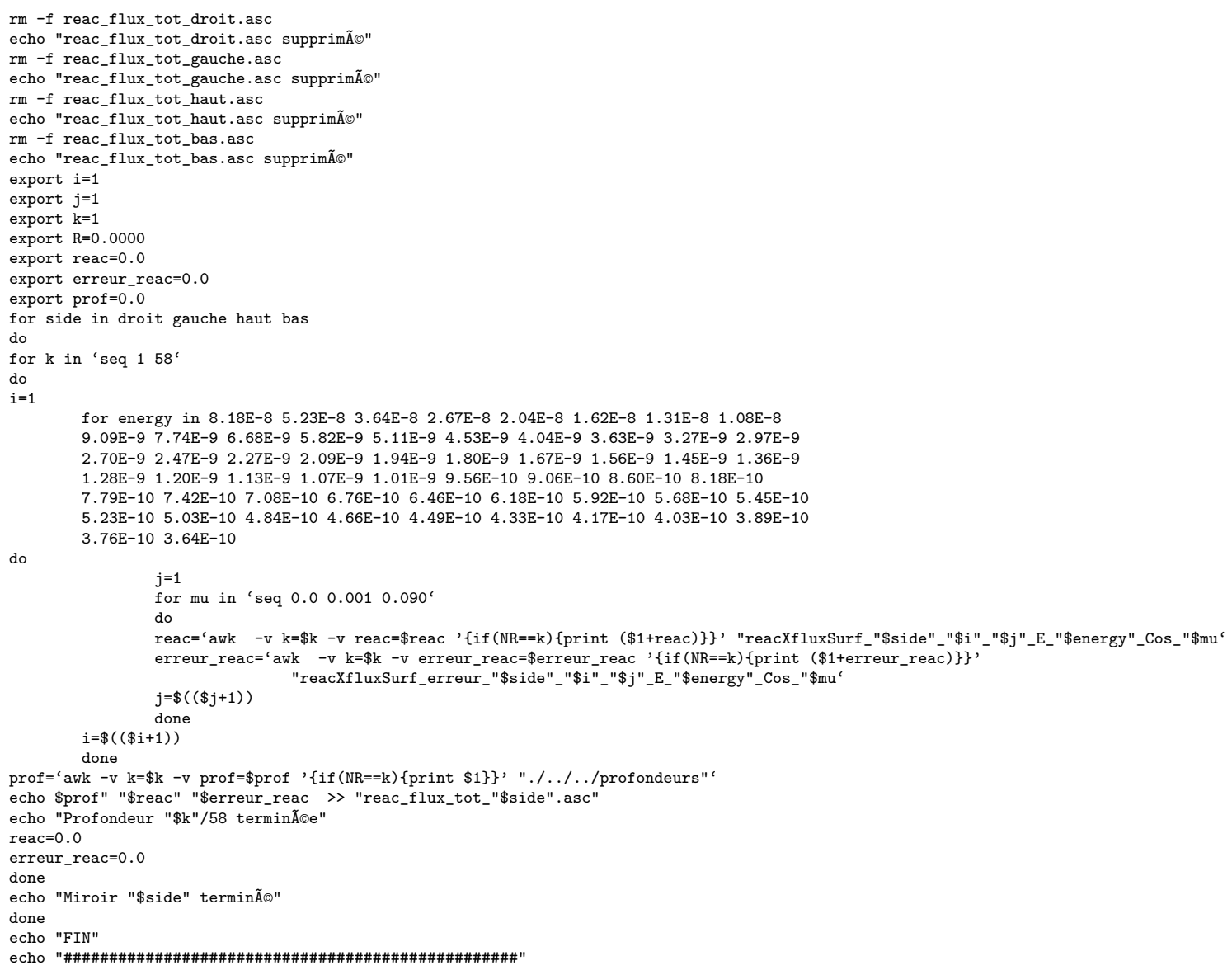




\section{Bibliography}

[1] AG3net. http://sfp.in2p3.fr/accelerateur/journees_2000/Roscoff2000/contenu/industriels/sdms/confvide.html.

[2] RRUFF Project. RRUFF Project - http://rruff.info/.

[3] S-BSL7. http://www.ohara-gmbh.com/e/katalog/d_s-bsl7_e.html.

[4] Schott.

[5] Standard test method for Knoop and Vvickers hardness of materials.

[6] A. Abbas, Y. Serruys, D. Ghaleb, J.M. Delaye, B. Boizot, B. Reynard, and G. Calas. Nuclear Instrumentation and Methods in Physics Research B, 166-167:445-450, 2000.

[7] C. L. Allred. Effect of radiation on silicon and borosilicate glass. PhD thesis, Massachusetts Institute of Technology, 2003.

[8] Clark L. Allred, Jeffrey T. Borenstein, and Linn W. Hobbs. Neutron irradiationinduced dimensional changes in mems glass substrates. Nuclear Instrumentation and Methods in Physics Research B, 264:66-72, 2007.

[9] I.S. Anderson, O.Scharpf, P. Hoghoj, and P. Ageron. Multilayers for optics. Journal of Neutron Research, 5:55-61, 1996.

[10] F. Angeli, T. Charpentier, D. de Ligny, and B. Cailleteau. Boron speciation in soda-lime borosilicate glasses containing zirconium. Journal of American Ceramic Society, 93(9):2693 - 2704, 2010.

[11] F. Angeli, O. Villain, S. Schuller, T. Charpentier, D. de Ligny, L. Bressel, and L. Wondraczek. Effect of temperature and thermal history on borosilicate glass structure. Physical Review B, 85(054110), 2012.

[12] F. Angeli, O. Villain, S. Schuller, S. Ispas, and T. Charpentier. Insight into sodium silicate glass structural organization by multinuclear NMR combined with firstprinciples calculations. Geochim. Cosmochim. Acta, 75:2453 - 2469, 2011.

[13] M. Antonini, A. Manara, and S. Buckley. Microstructural changes in irradiated silica based glasses. Radiation Effects, 65:55-61, 1982. 
[14] K. Awazu and H. Kawazoe. Strained Si-O-Si bonds in amorphous $\mathrm{SiO}_{2}$ materials: A family member of active centers in radio, photo, and chemical responses. Journal of Applied physics, 94(10), 2003.

[15] H.D. Bale, R.E. Shepler, and G.W. Gibbs. Journal of Applied Physics, 41:241, 1970.

[16] J Barton and C Guillemet. Le verre : Science et Technologie. EDP Sciences, 2005.

[17] N. Baydogan and A.B. Tugrul. Borosilicate glass for gamma irradiation fields. Solid State Sciences, 14:1692-1697, 2012.

[18] J. Beaucour, M. Kreuz, M. Boehm, R. Boffy, V. Cristiglio, B. Demé, L. Didier, P. Falus, P. Fouquet, R. Gandelli, Y. Gibert, B. Giroud, B. Jarry, P. Lachaume, S. Roux, F. Thomas, and A. Vorobiev. The H5 guide system - the latest innovative guide system at the ILL. Neutron News, 26(3), 2015.

[19] K. H. Beckurts and K. Wirtz. Neutron physics. Springer-Verlag, 1964.

[20] J. Blendell. PhD thesis, Massachusetts Institute of Technology, 1979.

[21] P. Blum. Opération "Remplacement nez de guides" - Projet 6010 - compte-rendu final. Technical report, Institut Laue Langevin, 1982.

[22] R. Boffy, M. Kreuz, J. Beaucour, U. Köster, and F.J. Bermejo. Why neutron guides may end up breaking down? Some results on the macroscopic behaviour of alkaliborosilicate glass support plates under neutron irradiation. Nuclear Instruments and Methods in Physics Research B, 358:179 - 187, 2015.

[23] R. Boffy, S. Peuget, R. Schweins, J. Beaucour, and F.J. Bermejo. High thermal neutron flux effects on structural and macroscopic properties of alkali-borosilicate glasses used as neutron guide substrate. EMRS spring meeting 2015 Proceedings, 2016.

[24] C. Bouton. Étude et réalisation de conduits de neutrons. PhD thesis, Faculté des sciences de l'université de Grenoble, 1970.

[25] P. J. Bray and J. G. O'Keefe. Nuclear magnetic resonance investigations of the structure of alkali borate glasses. Physics and Chemistry of Glasses, 4:37-46, 1963.

[26] R. Brückner, H.-U. Chun, H. Goretzki, and M. Sammet. Xps measurements and structural aspects of silicate and phosphate glasses. J. Non-Cryst. Solids, 42:49-60, 1980.

[27] B.C. Bunker, D.R. Tallant, R.J. Kirkpatrick, and G.L. Turner. Multinuclear nuclear magnetic resonance and Raman investigation of sodium borosilicate glass structure. Physics and Chemistry of Glasses, 31:30-41, 1990.

[28] J. Christ and T. Springer. Nukleonik, 4:23, 1962. 
[29] J. Clément, P. Torres, F.J. Gil, J.A. Planell, R. Terradas, and S. Martinez. Evaluation by Vickers indentation of Fracture toughness of a phosphate biodegradable glass. Journal of Materials Science Materials in Medicine, 10:437-441, 1999.

[30] Computing for Science Group. Virtual instruments and experiments.

[31] J. De Bonfils, S. Peuget, G. Panczer, D. de Ligny, S. Henry, P.-Y. Noël, A. Chenet, and B. Champagnon. Effect of chemical composition on borosilicate glass behavior under irradiation. J. Non-Cryst. Solids, 356(6-8):388-393, 2010.

[32] J.-M. Delaye, S. Peuget, G. Bureau, and G. Calas. Molecular dynamics simulation of radiation damage in glasses. Journal of non-crystalline solids, 357(14):2763-2768, 2011.

[33] W.J. Dell, P.J. Bray, and S.Z. Xiao. ${ }^{11} \mathrm{~B}$ NMR studies and structural modeling of $\mathrm{Na}_{2} \mathrm{O}-\mathrm{Bsbs}_{2} \mathrm{O}_{3}-\mathrm{SiO}_{2}$ glasses of high soda content. Journal of Non-Crystalline Solids, 58:1-16, 1983.

[34] R. G. Della Valle and E. Venutti. High-pressure densification of silica glass: A molecular-dynamics simulation. Physical Review B, 54(6):3809-3816, 1996.

[35] R.A.B. Devine. Macroscopic and microscopic effects of radiation in amorphous SiO2. Nuclear Instruments and methods in Physics Research B, 91:378-390, 1994.

[36] L.-S. Du and J. F. Stebbins. Network connectivity in aluminoborosilciate glasses: A high-resolution ${ }^{11} \mathrm{~B},{ }^{27} \mathrm{Al}$ and ${ }^{17} \mathrm{O}$ NMR study. Journal of Non-Crystalline Solids, 351:3508-3520, 2005.

[37] H. Eckert. Insulating and semiconducting glasses. World Scientific, 2000.

[38] N.A. El-Alaily and R.M. Mohamed. Effect of irradiation on some optical properties and density of lithium borate glass. MAterials Science and Engineering B, 98:193203, 2003.

[39] G. El-Damrawi, W. Müller Warmuth, H. Doweidar, and I.A. Gohar. 11B, 29Si and $27 \mathrm{Al}$ nuclear magnetic resonnance studies of $\mathrm{Na}_{2} \mathrm{O}-\mathrm{Al}_{2} \mathrm{O}_{3}-\mathrm{B}_{2} \mathrm{O}_{3}-\mathrm{SiO}_{2}$ glasses. Physics and Chemistry of Glasses, 34(2):52-57, 1993.

[40] S. R. Elliot. Solid State ionics, 105:39, 1998.

[41] T. H. Elmer, M. E. Nordberg, G. B. Carrier, and E. J. Korda. Phase separation in borosilicate glasses as seen by electron microscopy and scanning electron microscope. Journal of the American Ceramic Society, 53(4):171-175, 1970.

[42] I.L.L. Division Projets et Techniques. Note DPT 11 - distribution des neutrons. January 2008.

[43] M. Fábián, E. Sváb, Gy. Mészáros, Zs. Révay, Th. Proffen, and E. Veress. Network structure of multi-component sodium borosilicate glasses by neutron diffraction. Journal of Non-Crystalline Solids, 353(2084-2089), 2007. 
[44] S.P. Faile and D.M. Roy. Journal of American Ceramic Society, 49:638 - 643, 1966.

[45] E. Fermi and W.H. Zinn. Physical Review, 70:103, 1946.

[46] Enrico Fermi and L. Marshall. Interference phenomena of slow neutrons. Physical Review, 71:666, 1947.

[47] T. Furukawa and W. B. White. Raman spectroscopic investigation of sodium borosilicate glass structure. J. Mater. Sci., 16(10):2689-2700, 1981.

[48] Y. Y. Galakhov and V. T. Vavilonova. Liquid-phase separation in three-component borosilicate systems. Fizika $i$ Khimika Stekla, 11(3):276-281, 1985.

[49] L.F. Gladden, L.D. Pye, W.C. LaCourse, and H.J. Stevens. The physics of NonCrystalline Solids. Taylor and Francis, 1992.

[50] B. Gross. Irradiation effects in borosilicate glass. Physical Review, 107:368, 1957.

[51] A. Guinier and G. Fournet. Small-angle scattering of X-Rays. John Wiley and Sons, 1955.

[52] Thomas Harloff. Titanium allergy or not? "Impurity" of titanium implant materials. Health, 2:306-310, 2010.

[53] J.C. Hurt and C.J. Phillips. Structural role of zinc oxide in glasses in the system $\mathrm{Na}_{2} \mathrm{O}-\mathrm{ZnO}-\mathrm{SiO}_{2}$. Journal of the American Ceramic Society, 53(5):269-273, 1971.

[54] Y. Inagaki, H. Furuya, K. Idemitsu, T. Banda, S. Matsumoto, and S. Murakoa. Material research society symposium proceedings, 257:199-206, 1992.

[55] Joint meeting of the National Organization of Test, Research, and Training Reactors and the International Group on Research Reactors. Analysis of the Causes and Consequences of Neutron Guide Tube Failures, 2005.

[56] K.H. Kleno, K. Lieutenant, K.H. Andersen, and K. Lefmann. Systematic performance study of common neutron guide geometries. Nuclear Instrumentation and Methods in Physics Research B, 696:75-84, 2012.

[57] L. Koester. Neutron Physics, volume 80. Springer-Verlag, 1977.

[58] A.C. Komarek, P. Böni, and M. Braden. Parabolic versus elliptic focusing - Optimization of the focusing design of a cold triple-axis neutron spectrometer by MonteCarlo simulations. Nuclear Instruments and Methods in Physics Research Section A, 647:63-72, 2011.

[59] M. Kreuz. Guide specification. H5 guide system in ILL 5, November 2011.

[60] K. Lefmann and K. Nielsen. Mcstas, a general software package for neutron raytracing simulations. Neutron news, 10(3):20-23, 1999.

[61] P. Link. Neutron guides, not always perfect. FRM II news, 8:10, 2012. 
[62] J. Liouville. Journal de Mathématiques pures et appliquées, 1838.

[63] E. Lippmaa, M. Mägi, A. Smoson, and M. Tarmaka dn G. Engelhardt. Investigation of the structure of zeolites by solide-state high-resolution ${ }^{29} \mathrm{Si}$ NMR spectroscopy. Jornal of American Chemical Society, 103:4992-4996, 1981.

[64] Los Alamos National Laboratory. MCNP6.1.

[65] D. Lukàšik and P. Macko. Vickers hardness number of neutron irradiated chalcogenide ge-s glasses. J. Non-Cryst. Solids, 44:397-399, 1981.

[66] H. Maekawa, T. Maekawa, K. Kawamura, and T. Yokokawa. The structura groups of alkali silicate glasses determined from ${ }^{29} \mathrm{Si}$ MAS-NMR. Journal of NonCrystalline Solids, 127:53-64, 1990.

[67] H. Maier-Leibnitz and T. Springer. The use of neutron optical devices on beam-hole experiments. Journal of Nuclear Energy Parts A/B, 17:217-225, 1963.

[68] G. Malow and H. Andresen. Helium Formation from $\alpha$-Decay and its Significance for Radioactive Watte Glasses. Scientific Basis for Nuclear Waste Management, volume 1. Plenum Press, 1979.

[69] D. Manara, A. Grandjean, and D.R. Neuville. Advances in understanding the structure of borosilicate glasses: A Raman spectroscopy study. American Mineralogist, 94:777-784, 2009 .

[70] R. Martens and W. Müller-Warmuth. Structral groups and their mixing in borosilicate glasses of variour composition - an NMR study. Journal of Non-Crystalline Solids, 265(167-175), 2000.

[71] D. Massiot, F. Fayon, M. Capron, I. King, S. Le Calvé, B. Alonso, J-O Durand, B. Bujoli, Z. Gan, and G. Hoatson. Modelling one- and two-dimensional solid-state NMR spectra. Magnetic Resonance in Chemistry, 40:70-76, 2002.

[72] Dean W. Matson, Shiv K. Sharma, and John A. Philpotts. The structure of highsilica alkali-silicate glasses. A Raman spectroscopic investigation. Journal of NonCrystalline Solids, 58:323-352, 1983.

[73] Hj. Matzke and E. Vernaz. Journal of Nuclear Materials, 201:295 - 309, 1993.

[74] B.N. Meera and J. Ramakrishna. Raman spectral studies of borate glasses. J. Non-Cryst. Solids, 1993.

[75] C. Mendoza, S. Peuget, T. Charpentier, M. Moskura, R. Caraballo, O. Bouty, A.H. Mir, I. Monnet, C. Grygiel, and C. Jegou. Oxide glass structure evolution under swift heavy ion irradiation. Nuclear Instruments and Methods in Physics Research $B, 325: 54-65,2014$.

[76] C. Mylonas and R. Truell. Radiation effects from $(\mathrm{n}, \alpha)$ reactions in boron glass and energy of the racting neutrons. Journal of applied physics, 1958. 
[77] M. Olsen. Activation of color centers in glass. Technical report, Colorado State University, 2003.

[78] J. Paymal. Quelques effets des réactions $(\mathrm{n}, \alpha)$ dans les verres. Verres et Réfractaires, 6:341, 1961.

[79] J. Paymal and M. Bonnaud. Modification des verres sous l'influence des rayonnements. In Journées internationales d'études 1961 de l'Association belge pour favoriser l'étude des Verres et des Composés siliceux, pages 17 - 31, 1961.

[80] J. K. Percus and Yevick. J. Phys. Rev, 110(1), 1958.

[81] S. Peuget, J.-N. Cachia, C. Jégou, X. Deschanels, D. Roudil, V. Broudic, and J.M. Bart. Irradiation stability of R7T7-type borosilicate glass. Journal of nuclear materials, 354:1-13, 2006.

[82] S. Peuget, T. Fares, E.A. Maugeri, R. Caraballo, T. Charpentier, L. Martel, J. Somers, A. Janssen, T. Wiss, F. Rozenblum, M. Magnin, X. Deschanels, and C. Jégou. Effect of ${ }^{10} \mathrm{~B}(\mathrm{n}, \alpha) \mathrm{Li}$ irradiation on the structure of a sodium borosilicate glass. Nuclear Instrumentation and Methods in Physics Research B, 327:22-28, 2014.

[83] Sylvain Peuget, Emilio Andrea Maugeri, Thibault Charpentier, Clément Mendoza, Mélanie Moskura, Toby Fares, Olivier Bouty, and Christophe Jégou. Comparison of radiation and quenching rate effects on the structure of a sodium borosilicate glass. Journal of Non-Crystalline Solids, 378:201-212, 2013.

[84] A. Pilkington. Float: an application of science, analysis, and judgement. Glass Technology, 12(4), 1971.

[85] I. G. Polyakova. Alkali borosilicate systems: diagrams and properties of glasses. Physics and Chemistry of glasses, 41(5):247 - 258, 2000.

[86] M.O. Prado, A.A. Campos Jr., P.C. Soares, A.C.M. Rodrigues, and E.D. Zanotto. Liquid-liquid phase separation in alkali-borosilicate glass. an impedance spectroscopy study. Journal of non-crystalline solids, 332:166-172, 2003.

[87] M.O. Prado, N.B. Messi, T.S. Plivelic, I.L. Torriani, A.M. Bevilacqua, and M.A. Arribére. The effects of radiation on the density of an aluminoborosilicate glass. $J$. Non-Cryst. Solids, 289:175-184, 2001.

[88] W. Primak. Journal of Applied Physics, 1958.

[89] S. Pullen, G. Davidson, S. Pangalis, F. Klose, and S. Kennedy. Report on the repair of the opal neutron beam transport system. In Joint IGORR 2013 and IAEA Technology Meeting, 2013.

[90] J Rault. Ageing of glass: role of the Vogel-Fulcher-Tamman law. J. Phys.: Condens. Matter, 15:1193-1213, 2003. 
[91] J.M. Roderick, D. Holland, and C.R. Scales. Characterization and radiation resistance of a mixed-alkali borosilicate glass for high-level waste vitrification. MRS Proceedings, 608:721 - 726, 2000.

[92] A. B. Rosenthal and S. H. Garofalini. Structural role of zinc oxide in silica and soda-silica glasses. Journal of American Ceramic Society, 70(11):821-826, 1987.

[93] M. Sacchi, M. Anttonini, S.N. Buckley, and A. Manara. Alkali effects on bubble formation in irradiated borosilicate glasses. Materials Letters, 4(1):10-12, 1985.

[94] SasView. http://www.sasview.org/.

[95] U. Schmidt, D. Dubbers, K. Raum, O. Joeres, and O. Scharpf. Supermirror beam bender and concentrator for slow neutrons. Journal of Neutron Research, 5:81-88, 1996.

[96] Scholze. Glas, Natur, Struktur und Eigenschaften. Springer, 1977.

[97] Schott. Borofloat. http://www.schott.com/borofloat/english/.

[98] V. F. Sears. Neutron Optics : An Introduction to the Theory of Neutron Optical Phenomena and Their Applications. Oxford University Press, 1989.

[99] Varley F. Sears. Neutron scattering lengths and cross sections. Neutron news, 3(3):29-37, 1992.

[100] J.E. Shelby. Effect of radiation on the physical properties of borosilicate glasses. Journal of applied physics, 51(5):2561-2565, 1980.

[101] H. A. Sheybany. De la structure des verres alumino silicatés mixtes. Verres Réfractaires, 2:127, 1948.

[102] J. Shneider, V.R. Mastelaro, E.D. Zanotto, B. A. Shakhmatkin, N. M. Vedishcheva, A. C. Wright, and H. Panepucci. $Q^{n}$ distribution in stoichiometric silicate glasses: thermodynamic calculations and ${ }^{29} \mathrm{Si}$ high resolution NMR measurements. J. NonCryst. Solids, 325:164-178, 2003.

[103] I. Simon. Structure of neutron-irradiated quartz and vitreous silica. Journal of the American Ceramic Society, 40(5):150-153, 1957.

[104] Michel Soutif. Physique Neutronique. Presses Universitaires de France, 1962.

[105] G. G. Stoney. The tension of metallic films deposited by electrolysis. Proceedings of the Royal Society of London, pages 1905-1934, 1909.

[106] Swiss Neutronics. http://www.swissneutronics.ch.

[107] Andreas Van-Overberghe. High Flux Neutron Imaging for highly dynamic and time resolved non-destructive testing. PhD thesis, University of Heidelberg, 2006.

[108] S. Weissmann and K. Nakajima. Journal of Applied Physics, 34:611, 1963. 
[109] P. Willendrup, E. Farhi, and K. Lefmann. Mcstas 1.7 - a new version of the flexible Monte Carlo neutron scattering package. Physica B: Condensed Matter (Amsterdam, Netherlands), 350:735-737, 2004.

[110] Peter Kjaer Willendrup, Emmanuel Farhi, U. Filges, and Kim Lefmann. Component Manual for the Neutron Ray-tTracing Package McStas, Version 2.1, 2014.

[111] C. E. Williams, R. P. May, and A. Guinier. Materials science and technology: characterization of materials. Small-Angle Scattering of X-rays and Neutrons, volume 2 B. Wiley-VCH, 2005.

[112] R. N. Wilson. Reflecting telescope optics II. Springer, 1999.

[113] C. G. Windsor. Pulsed neutron scattering. Halsted Press, 1981.

[114] A. C. Wright, G. Dalba, F. Rocca, and N. M. Vedishcheva. Borate versus silicate glasses: why are they so different? Physics and Chemistry of Glasses: European Journal of Glass Science and Technology Part B, 51(5):233-265, 2010.

[115] A.C. Wright. Insulating and Semi-Conducting Glasses. P. Boolchand, 2000.

[116] A.C. Wright, B. Bachra, T.M. Brunier, R.N. Sinclair, L.F. Gladden, and R.L. Portsmouth. A neutron diffraction and MAS-NMR study of the structure of fast neutron irradiated vitreous silica. Journal of Non-Crystalline Solids, 150:69-75, 1992.

[117] M. Yamane and J.D. Mackenzie. Vicker's hardness of glass. J. Non-Cryst. Solids, 15:153-164, 1974.

[118] K.J. Yang, T.S. Wang, G.F. Zhang, H.B. Peng, L. Chen, L.M. Zhang, C.X. Li, F. Tian, and W. Yuan. Study of irradiation damage in borosilicate glass induced by He ions and electrons. Nuclear Instrumentation and Methods in Physics Research B, 307:541-544, 2013.

[119] Y.H. Yun and P.J. Bray. Nuclear magnetic resonance studies of glass in the system $\mathrm{Na}_{2} \mathrm{O}-\mathrm{B}_{2} \mathrm{O}_{3}-\mathrm{SiO}_{2}$. J. Non-Cryst. Solids, 27:363-380, 1978.

[120] A. Zeidler, P. S. Salmon, H. E. Fischer, J. C. Neuefeind, J. M. Simonson, H. Lemmel, H. Rauch, and T. E. MArkland. Physical Review Letter, 107, 2011.

[121] J.F. Ziegler and J.M. Manoyan. The stopping of ions in compounds. Nuclear Instruments and Methods in Physics Research B, 35:215-228, 1988. 




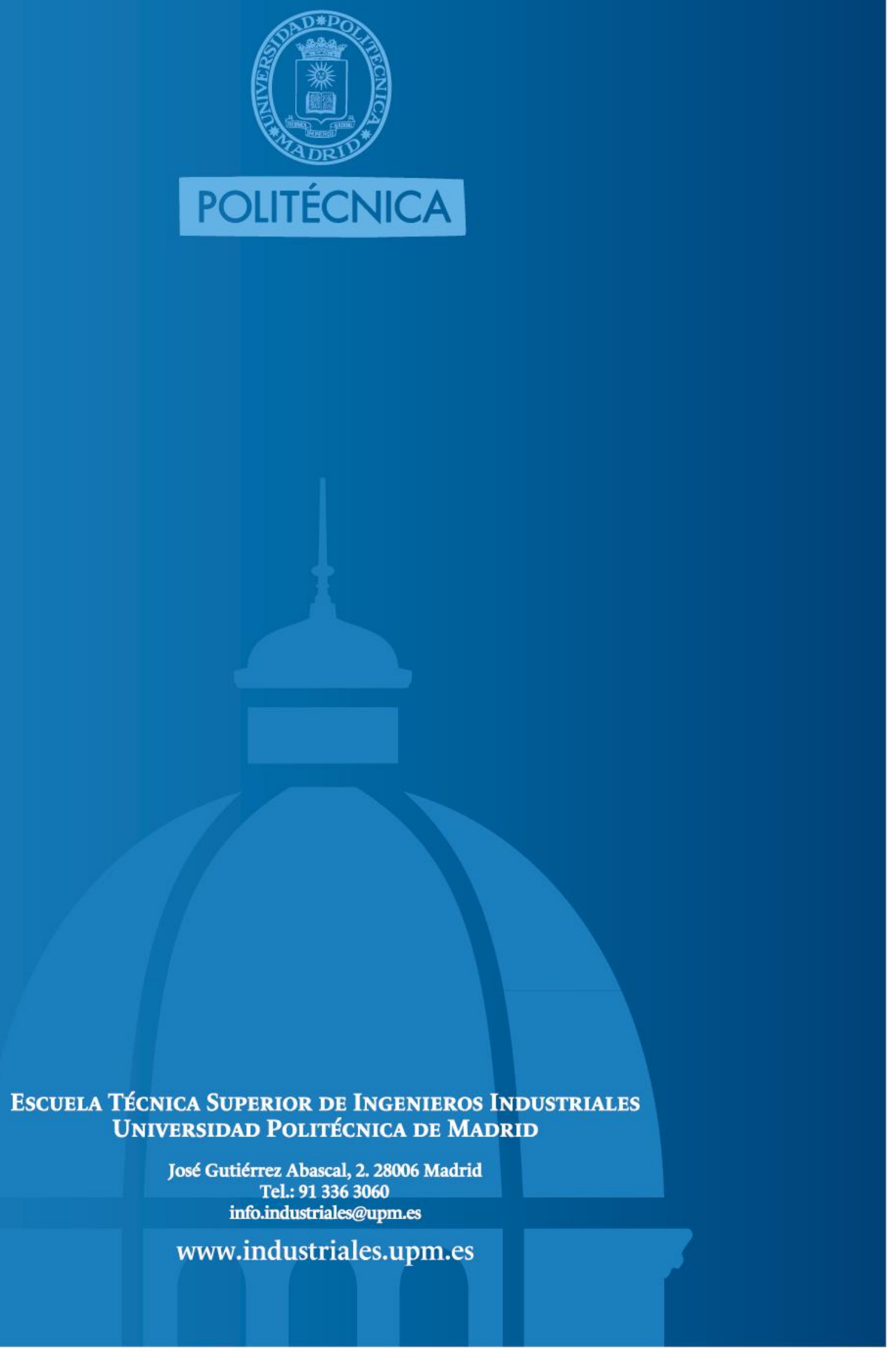